\title{
$\beta$-Peptide Helices As Transmembrane Domains: Aggregation, Recognition and Lipid-Peptide Interaction
}

\section{Dissertation}

zur Erlangung des mathematisch-naturwissenschaftlichen Doktorgrades

" Doctor rerum naturalium " der Georg-August-Universität Göttingen

im Promotionsprogramm Chemie der Georg-August-University School of Science (GAUSS)

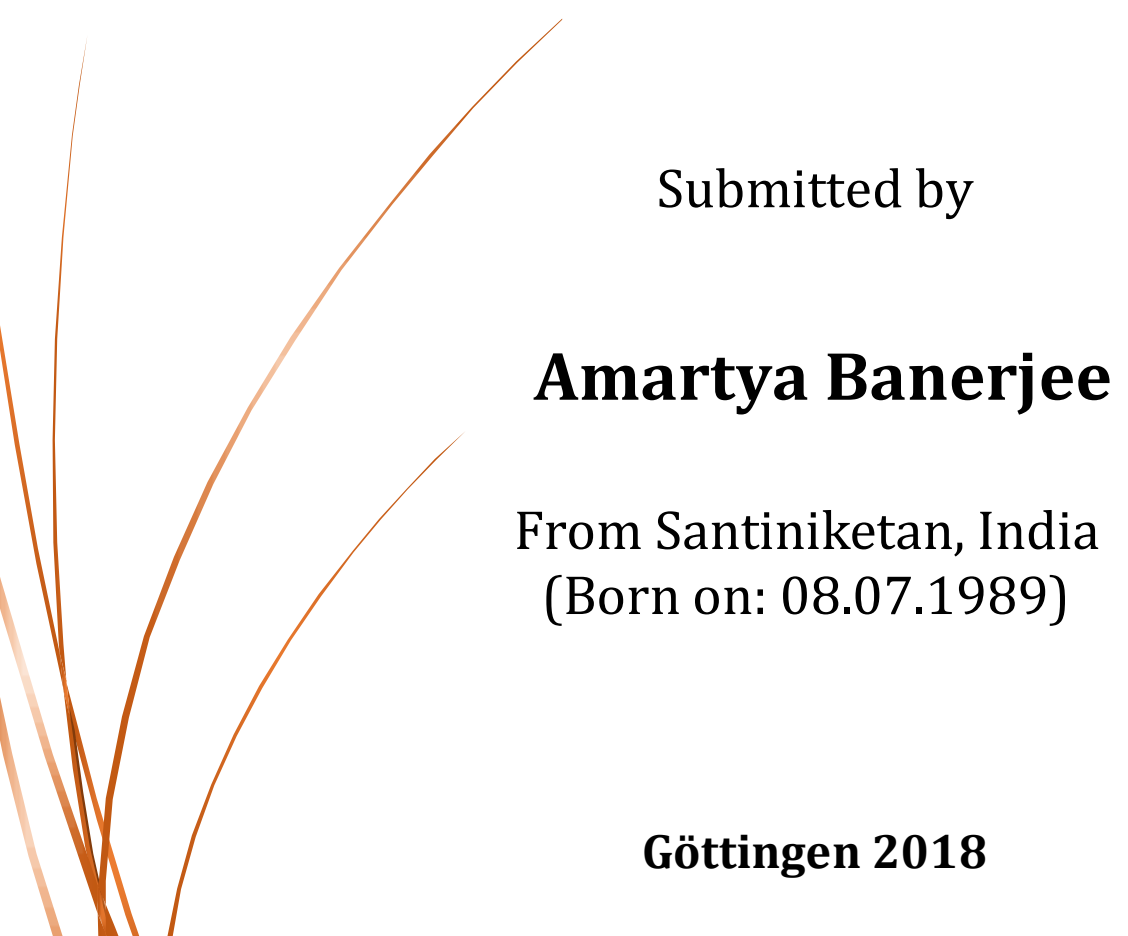




\section{Thesis Committee}

Prof. Dr. Ulf Diederichsen

Institute of Organic und Biomolecular Chemistry, Georg-August University Göttingen

Prof. Dr. Claudia Steinem

Institute of Organic und Biomolecular Chemistry, Georg-August University Göttingen

\section{Members of the Examination Commission}

\section{Reviewer-1}

Prof. Dr. Ulf Diederichsen

Reviewer -2

Prof. Dr. Claudia Steinem
Institute of Organic und Biomolecular Chemistry, Georg-August University Göttingen

Institute of Organic und Biomolecular Chemistry, Georg-August University Göttingen

\section{Further Members of the Examination Commission}

Prof. Dr. Kai Tittmann

Prof. Dr. Inke Siewert

Prof. Dr. Rolf Daniel

Dr. Holm Frauendorf

$$
\begin{gathered}
\text { Schwann-Schleiden-Forschungszentrum } \\
\text { Dept. of Molecular Enzymology } \\
\text { Georg-August University, Göttingen } \\
\text { Institute of Inorganic Chemistry } \\
\text { Georg-August University, Göttingen }
\end{gathered}
$$
Genomische und Angewandte Mikrobiologie
\& Göttingen Genomik Labor Georg-August University Göttingen

Institute of Organic \& Biomolecular Chemistry Georg August University, Göttingen 
Dedicated To.

My beloved idol, Dada (Grandfather), Prof. Dr.rer.nat Ait K. Banerjee (Göttíngen) 


\section{Abstract}

Plasma membranes are considered as the primary regulatory unit essential for efficient functioning of a cell. These membranes separate the inner part of the cell from the extracellular world as well as acts as a barrier selectively regulating the passage across it. The plasma membranes are composed of various different constituents, but among all, membrane proteins are unanimously accepted as one of the major structural and functional pillars of the plasma membranes by the scientific community. In course of scientific research, malfunctioning of the membrane proteins have been found to be responsible for serious diseases. So, it has been of great scientific interest to design and develop artificial transmembrane protein domains that are able to address the shortcomings of the natural ones. Understanding the molecular forces and other physico-chemical processes behind the folding conformation and transmembrane dynamics of these protein domains are an integral part in the process of developing updated synthetic transmembrane protein domains.

During last two decades, evolution of $\beta$-peptides as one of the more promising peptidomimetic motifs has been at large. The extraordinary stability against proteolytic degradation in presence of proteases and ability to generate stable helical secondary structures, such as, 1412- and alternate 10/12-helices, with as few as 4-6 amino acids are the two main reasons behind the booming entry of $\beta$-peptides into peptidomimetics. Besides all other important parameters, the helical macro-dipole moment of peptides is thought to have significant role in transmembrane insertion and spanning. Direct experimental determination of helical macrodipole moment of peptides being extremely challenging, the possible effects of helical macrodipole moment has only been theoretically proposed. Therefore, one of the major objectives of this thesis is to shed light on any possible role of helical macro-dipole moment in transmembrane insertion and spanning as well as in cellular uptake via indirect experiments.

In general, $\beta$-peptides possess definite helical macro-dipole moment but in opposite direction as compared to that of the naturally occurring $\alpha$-peptide analogues. The idea is to locate and synthesize a type of $\beta$-peptide that has almost no overall helical macro-dipole moment and perform transmembrane insertion studies with the $\beta$-peptides with and without helical macrodipole keeping all other parameters constant. In case, they exhibit any differential ability for membrane insertion, it would experimentally indicate the role of helical macro-dipole in the respective physico-chemical phenomenon. After extensive research, alternate 10/12-helical $\beta$ peptides composed of alternate $\beta^{3} / \beta^{2}$-amino acids was found to have overall nullified helical 
macro-dipole due to their unique conformational orientation. Therefore, different types of $\beta$ peptides including 14-, 12- and alternate 10/12-helical peptides with comparable transmembrane length and sequence are planned to be synthesized by mixing different synthetic strategies, such as, microwave-assisted manual SPPS, non-microwave-assisted manual SPPS, and fluorous-tag-attached liquid phase peptide synthesis. As the next step, transmembrane insertion of the peptides would be checked by hydrophobic microenvironment sensitive Trp-fluorescence spectroscopy. A same group of three different lipids, DLPC/DMPC/POPC, is chosen for all the different 14-, 12- and alternate 10/12-helical peptides with comparable lengths in such a way that negative hydrophobic mismatch is induced gradually starting from an almost perfectly hydrophobic matching situation. It is well known that above a threshold value of negative hydrophobic mismatch, transmembrane insertion is not feasible. On the other hand, short-chain alcohols, like ethanol, is known to possess a pronounced effect in reducing the lipid membrane thickness by interdigitating the acyl chains. Therefore, a gradual increased concentration of EtOH would be used for the different peptides and the minimum threshold concentration of $\mathrm{EtOH}$ required for each peptides for the same lipid with similar negative hydrophobic mismatch would carefully be monitored to have a quantitative estimation on the minimum compensation of negative mismatch required for successful transmembrane insertion with the help of Trp-fluorescence spectroscopy. From this experimental results, it would be possible to conclude that the peptide that requires more minimum concentration of $\mathrm{EtOH}$, that is, higher compensation of negative mismatch, to successfully get reconstituted into lipid membranes, are less prone towards transmembrane insertion and spanning. In case, the peptides with and without helical macro-dipole moment exhibit any differential propensity towards this behavior, it would indirectly indicate and experimentally verify significant role of helical macro-dipole moment in transmembrane insertion and spanning (since the main difference between the peptides is the presence and absence of helical macro-dipole moment).

Moreover, the secondary helical structures of all the different peptides would be determined in solution as well as inside lipid vesicles to elucidate whether the characteristic helical pattern is maintained when inside lipid environment. It would also be checked whether the stability of 14- and 10/12-helical peptides is altered when inside lipid vesicles in comparison to that in solution by temperature dependent CD-spectroscopy. This would also indicate if the helical macro-dipole moment has any effect in stabilizing the secondary helical structures in solution or inside lipid vesicles.

Finally, 6-amino acid long short chain 14-helical and 10/12-helical 5(6)-FAM attached peptides are synthesized for cellular uptake studies using human bronchial adenocarcinoma cell lines 
A549. First the cytotoxicity is checked on the same cell line by clonogenic assay and MTT-assay. If found non-cytotoxic until $1 \mu \mathrm{M}$ concentration, further studies towards quantitative cellular uptake efficiencies by fluorescence activated cell sorting (FACS) is carried out on 14- and alternate 10/12-helical peptides. A well-reputed cell penetrating peptide, HIV-1 TAT, is used as a reference standard. Any difference in cellular uptake efficiencies between the two target peptides would experimentally indicate that the helical macro-dipole moment not only affects transmembrane insertion and spanning but also regulates cellular uptake. To ensure and support the FACS results, the peptides would also be visualized under confocal laser scanning fluorescence microscopy. The microscopy imaging would exhibit whether the target peptides indeed are internalized via cell penetration or are only stuck to the membranes. Additionally, if any of the target $\beta$-peptides were found to have significant cell penetration potency, it would open a new spectrum towards developing novel, hydrophobic, uncharged cell penetrating peptide (CPP) candidates that are stable in presence of proteases.

Finally, all these studies would experimentally shed light on the regulatory effect of the helical macro-dipole moment of membrane protein domains in transmembrane insertion, spanning and in cellular uptake. This information would address the effect of peptide helical macrodipole moment in these important physico-chemical phenomena and help in designing $\beta$ peptide based model transmembrane domain systems as well as $\beta$-peptide-based new generation cell penetrating peptides. 


\section{Table of Contents}

1. Lipid Membranes 1

1.1 General 1

1.2 Membrane Structure and Components 1

$\underline{1.3 \text { Membrane Lipids }} \quad 3$

1.3.1 Phospholipids $\quad 4$

1.3.2 Glycolipids $\quad 4$

1.3.3 Cholesterol 5

1.3.4 Lipid Dynamics \& Flip-Flop

$\begin{array}{ll}\text { 1.3.5 Membrane Fluidity } & 7\end{array}$

1.4 Membrane Proteins $\quad 9$

1.5 Protein-Lipid Interaction in Biological membrane $\quad 10$

1.6 Effect of EtOH in Compensating Negative Hydrophobic

Mismatch by Reduction of Bilayer Thickness 12

2. $\beta$-Peptide: A Model Synthetic Transmembrane Domain 14

$\begin{array}{ll}2.1 \text { General } & 14\end{array}$

$\begin{array}{ll}2.2 \text { Evolution of } \beta \text {-Peptides } & 18\end{array}$

$\begin{array}{ll}2.2 .1 \text { General } & 18\end{array}$

2.2.2 Major Helical Secondary Structures of $\beta$-Peptides $\quad 19$

2.2.3 Peptide Helical Macro-dipole Moment 25

3. $\beta$-Peptide: Design $\quad 28$

$\begin{array}{ll}3.1 \text { General } & 28\end{array}$

$\underline{3.2 \text { Structural Characteristics of Transmembrane } \alpha \text {-Helices in }}$

Membrane Spanning and at Membrane-Water Interface Region 29

$\underline{3.3 \text { Design of 10/12-Helical } \beta \text {-Peptide Based Artificial }}$

$\begin{array}{ll}\text { Transmembrane Domains } & 32\end{array}$

3.4 Design of 14-Helical $\beta$-Peptide Based Artificial 
3.5 Design of 12-Helical $\beta$-Peptide Based Artificial

$\underline{\text { Transmembrane Domains }}$

4. $\beta$-Peptide: Synthesis

4.1 $\beta$-Amino Acids: A Brief Overview

$\underline{4.2 \text { Synthesis of } \beta^{3} \text {-Amino Acids }}$

4.3 Synthesis of $\beta^{2}$-Amino Acids

4.3.1 Route-1: Proline-Catalyzed Diastereoselective

Aminomethylation of Aldehydes

4.3.2 Route-2: Asymmetric $\alpha$-Alkylation of $\beta$-Alanine

Using Pseudoephedrine as a Chiral Auxiliary

4.4 Synthesis of 14-Helical $\beta$-Peptides $\quad 46$

4.4.1 Synthesis of 14-Helical $\beta^{3}$-Val Peptide $\quad 46$

4.4.2 Synthesis of 14-Helical $\beta^{3}$-(Val/Ala/Leu)-Triad Peptide 47

$\underline{4.5 \text { Synthesis of } 12 \text {-Helical } \beta \text {-Peptides }}$

$\underline{4.6 \text { Synthesis of Alternate 10/12-Helical } \beta \text {-Peptides }} \quad 50$

4.6.1 Heavy Fluorous-Tagged Solution Phase Synthesis $\quad 50$

4.6.2 Non-Microwave-Assisted Manual SPPS

$\underline{4.7 \text { Attachment of Fluorophores to Short-Chain } \beta \text {-Peptides }} 55$

4.8 Synthesized $\beta$-Peptides: AT-A-Glance $\quad 56$

$\underline{\text { 5. Results and Interpretation }} \quad 57$

$\underline{5.1 \text { Fluorescence Spectroscopy Analysis of } \beta \text {-Peptides: }}$

Transmembrane Peptide Insertion into Lipid Bilayers $\quad 57$

$\underline{5.2 \text { CD-Spectroscopic Analysis of } \beta \text {-Peptides }} \quad 70$

5.2.1 Fundamentals of CD-Spectroscopy

5.2.2 Secondary Structures of Target

$\beta$-Peptides in Solution 


\subsubsection{Secondary Structures of Target}

$\beta$-Peptides in Lipid Vesicles

6. $\beta$-Peptides: An Outlook Towards Cell Penetration

and Possible Effect of Macro-Dipole Moment 84

$\underline{6.1 \text { General }} \quad 84$

6.2 Cellular Uptake Mechanism: A Brief Overview 86

6.2.1 Inverted Micelle Formation $\quad 86$

6.2.2 Adaptive Translocation $\quad 87$

6.2.3 Pore Formation $\quad 88$

$\begin{array}{ll}\text { 6.3 Design } & 89\end{array}$

$\underline{6.4 \text { Results }} \quad 92$

6.4.1 Cytotoxicity Tests by Clonogenic Assay 92

6.4.2 Quantitative Cellular Uptake: Fluorescence

Activated Cell Sorting Assay (FACS) Study 95

6.4.3 Visualization: A Confocal Laser Scanning

Fluorescence Microscopy Imaging Study 106

6.4.3.1 5-FAM-HIV-1 TAT Peptide 107

$\begin{array}{ll}\text { 6.4.3.2 5(6)-FAM- } \beta^{3} \text {-Peptides (P7) } & 109\end{array}$

6.4.3.3 5(6)-FAM- $\beta^{2} / \beta^{3}$-Peptides (P9) 112

$\begin{array}{ll}\text { 7. Conclusion } & 115\end{array}$

$\begin{array}{lr}\text { 8. Experimental Section } & 122\end{array}$

$\begin{array}{ll}8.1 \text { General } & 122\end{array}$

$\begin{array}{ll}\text { 8.2 Characterization } & 126\end{array}$

8.2.1 Nuclear Magnetic Resonance (NMR) Spectroscopy 126

$\begin{array}{ll}\text { 8.2.2 Mass Spectroscopy } & 126\end{array}$

$\begin{array}{ll}\text { 8.2.3 Fluorescence Spectroscopy } & 126\end{array}$

$\begin{array}{ll}\text { 8.2.4 UV/VIS Spectroscopy } & 127\end{array}$ 
8.2.6 Circular Dichroism Spectroscopy (CD) 128

$\begin{array}{ll}\text { 8.2.7 Gas Chromatography (GC) } & 129\end{array}$

8.2.8 Gas Chromatography Mass Spectroscopy (GC-MS) 129

$\begin{array}{ll}\text { 8.3 General Procedures (G.P) } & 129\end{array}$

8.3.1 General Procedure to Synthesize $\beta^{3}$-D- Amino Acids $\quad 129$

$\begin{array}{ll}\text { 8.3.1.1 G.P-1 } & 129\end{array}$

$\begin{array}{ll}\text { 8.3.1.2 G.P-2 } & 130\end{array}$

8.3.2 General Procedures to Synthesize $\beta$-Peptides $\quad 131$

$\begin{array}{ll}\text { 8.3.2.1 G.P-3 } & 131\end{array}$

$\begin{array}{lr}\text { 8.3.2.2 G.P-4 } & 132\end{array}$

$\begin{array}{ll}\text { 8.3.2.3 G.P-5 } & 132\end{array}$

$\begin{array}{ll}\text { 8.3.2.4 G.P-6 } & 133\end{array}$

$\begin{array}{ll}\text { 8.3.2.5 G.P-7 } & 133\end{array}$

$\begin{array}{ll}\text { 8.3.2.6 G.P-8 } & 134\end{array}$

$\begin{array}{ll}\text { 8.3.2.7 G.P-9 } & 135\end{array}$

$\begin{array}{ll}\text { 8.3.2.8 G.P-10 } & 135\end{array}$

$\begin{array}{ll}\text { 8.3.2.9 G.P-11 } & 135\end{array}$

$\begin{array}{ll}\text { 8.3.2.10 G.P-12 } & 136\end{array}$

$\begin{array}{ll}\text { 8.3.2.11 G.P-13 } & 136\end{array}$

8.3.3 General Procedure to Prepare Peptide/Lipid Vesicles $\quad 138$

$\begin{array}{ll}\text { 8.3.3.1 G.P-16 } & 138\end{array}$

$\begin{array}{ll}\text { 8.3.3.2 G.P-17 } & 138\end{array}$

8.3.4 General Procedures for in vitro Studies of $\beta$-Peptides

As Potent Cell Penetrating Peptide Candidates on

$\begin{array}{ll}\text { Human Malignant Cell Lines } & 139\end{array}$

$\begin{array}{ll}\text { 8.3.4.1 G.P-18 } & 139\end{array}$ 



\section{Lipid Membranes:}

\subsection{General}

To stay alive, all living things require membranes. Membrane are the barriers that give cells their outer boundary and their inner compartments. Membranes are selective towards allowing molecules in and out of the cell and this selective permeability regulates the composition of the fluids within individual cells. Membrane control the flow of inter-cellular information either by sending chemical or electrical signals or by the recognition of signal molecules. Membranes are also involved in capture and release of energy. Diseases are found to be membrane-mediated, too [1]. Consequently, the importance of biological membranes in living systems cannot be overestimated. Therefore, biological membranes are much more than an inert barrier for protection, but they also play crucial part every events through the life cycle of a cell - from proliferation to senescence.

\subsection{Membrane Structure and Components}

Historically Ernest Overton had first hypothesized that cell membranes are made out of lipids.[1] Then in 1925, Evert Gorter and François Grendel found that red blood cell membranes are formed by a fatty layer two molecules thick, i.e. they described the bilipid nature of the cell membrane. ${ }^{[2]}$ A decade later in 1935, Danielli et al. postulated that the a layer of peripheral proteins remain tightly linked to the polar head groups of the phospholipids. ${ }^{[3]}$ Afterwards, in 1972 Singer et al. gave the detailed fluid mosaic model of cell membrane, which proposed the cell membrane as a two-dimensional liquid that restricts the lateral diffusion of membrane components. Such domains are defined by the existence of regions within the membrane with special lipid and protein composition that promote the formation of lipid rafts or protein and glycoprotein complexes. ${ }^{[4]}$ The main fabric of the membrane is composed of amphiphilic or dualloving, phospholipid molecules. The hydrophilic or water-loving areas of these molecules are in contact with the aqueous fluid both inside and outside the cell. Hydrophobic, or water-hating molecules, tend to be non- polar. A phospholipid molecule consists of a three-carbon glycerol backbone with two fatty acid molecules attached to carbons 1 and 2, and a phosphate-containing group attached to the third carbon. This arrangement gives the overall molecule an area described as its head (the phosphate-containing group), which has a polar character or negative charge, and an area called the tail (the fatty acids), which has no charge (Figure 1.1). The tail part composed of fatty acids usually interact with other non-polar molecules in chemical reactions, but generally do not interact with polar molecules. When placed in water, hydrophobic 
molecules self-aggregate and tend to form a ball or cluster like arrangement. The hydrophilic regions of the phospholipids tend to form hydrogen bonds with water and other polar molecules on both the exterior and interior of the cell and shields the hydrophobic tails from the direct contact of water. Thus, the membrane surfaces that face the interior and exterior of the cell are hydrophilic. In contrast, the middle of the cell membrane is hydrophobic and does not interact with water while being shielded by the hydrophilic headgroups. Therefore, phospholipids form an excellent lipid bilayer cell membrane that separates fluid within the cell from the fluid outside of the cell (Figure 1.1). Such bilayers close on themselves to form sealed compartments known to be liposomes. The liposomes

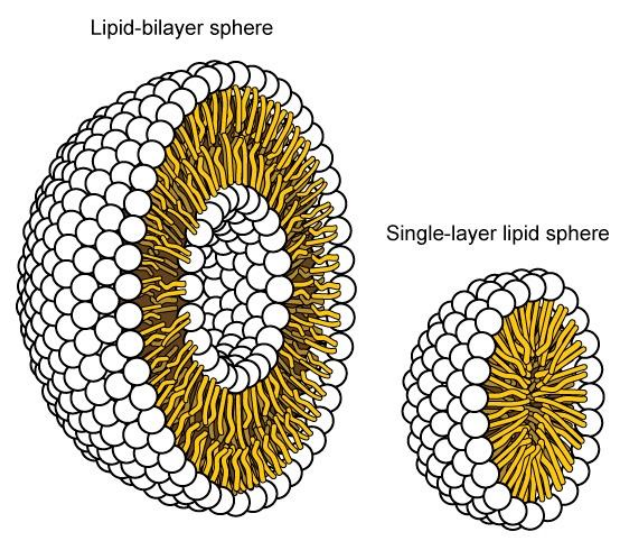

A

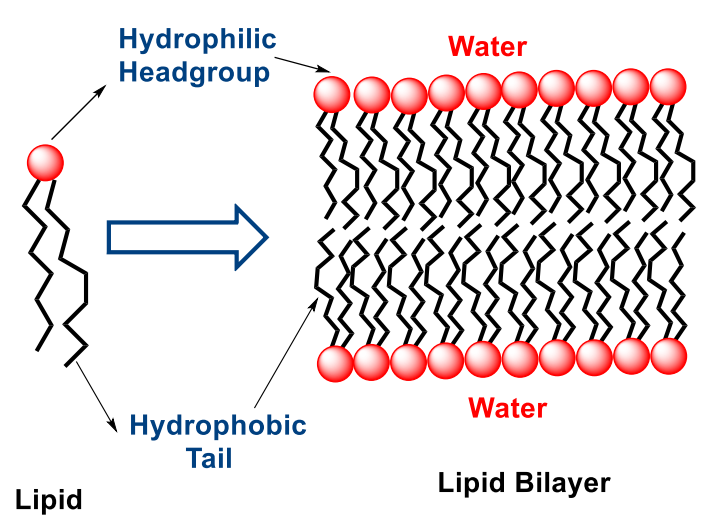

B

Figure 1.1: Arrangement of single-layer lipid sphere and bilayer lipid sphere in presence of aqueous solution (A); The amphiphilic nature of lipids determines their self-assembly. In aqueous environment, lipids assemble into a lipid bilayer (on the right), with their headgroups facing the aqueous phase and their hydrophobic tails facing each other, to minimize the contact between the tails and water (B) 
The thickness of lipid bilayers is about $5 \mathrm{~nm}$, with a hydrophobic core of about $3 \mathrm{~nm}$ formed by the hydrophobic tails. These lipid bilayers incorporate membrane proteins to carry out biological functions. There are two major classes of membrane proteins: integral membrane proteins, in which some domains of the proteins span across the $5 \mathrm{~nm}$ thick lipid bilayers, and peripheral membrane proteins, in which the proteins are only bound to one side of lipid membranes, either by binding to other proteins in membranes or by absorbing directly to lipid headgroups. The schematic diagram of a biological membrane is shown in Figure 1.2.

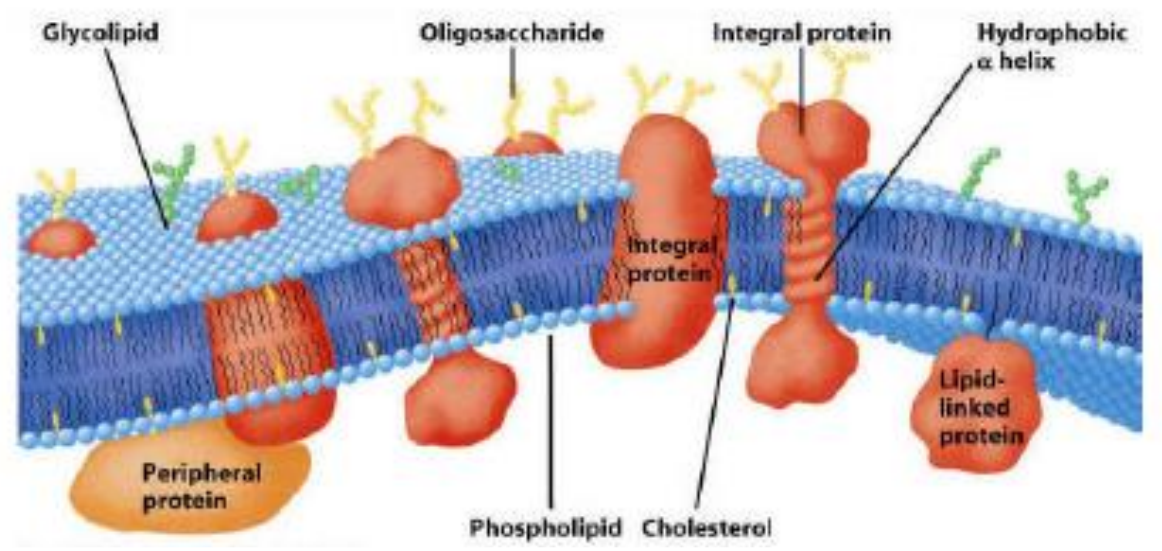

Figure 1.2: A detailed schematic representation of biological membrane

Major components of biomembranes including phospholipids, which have a phosphate in their headgroups, glycolipids, which have a carbohydrate attached to the headgroups, cholesterol, sphingomyelin, integral membrane proteins and peripheral membrane proteins. The compositions of biological membranes are tightly regulated by cellular machinery for performing vital biological processes.

\subsection{Membrane Lipids}

Lipids are biologically important substances that are immiscible in hydrophilic substances, like, water and miscible in hydrophobic organic solvents, such as, propanone, chloroform, diethyl ether, etc. Three major types of lipids are found in biological membranes: phospholipids, glycolipids and cholesterol. Each of these lipids has their own crucial roles in membrane function. 


\subsubsection{Phospholipids}

The most abundant type of phospholipids contain glycerol linked to two fatty acid chains, phosphate and choline. The fatty acid chains usually consists from 14 to 24 carbon atoms. One of the chains is generally unsaturated, containing one to four cis double bonds. Each double bond generates a bend in the fatty acid chain. Since, these lipids contain glycerol, so they are also known as glycero-phospholipids. Another type of phospholipids replace glycerol by sphingosine. A common example of such type of phospholipid is sphingomyelin (Figure 1.3).
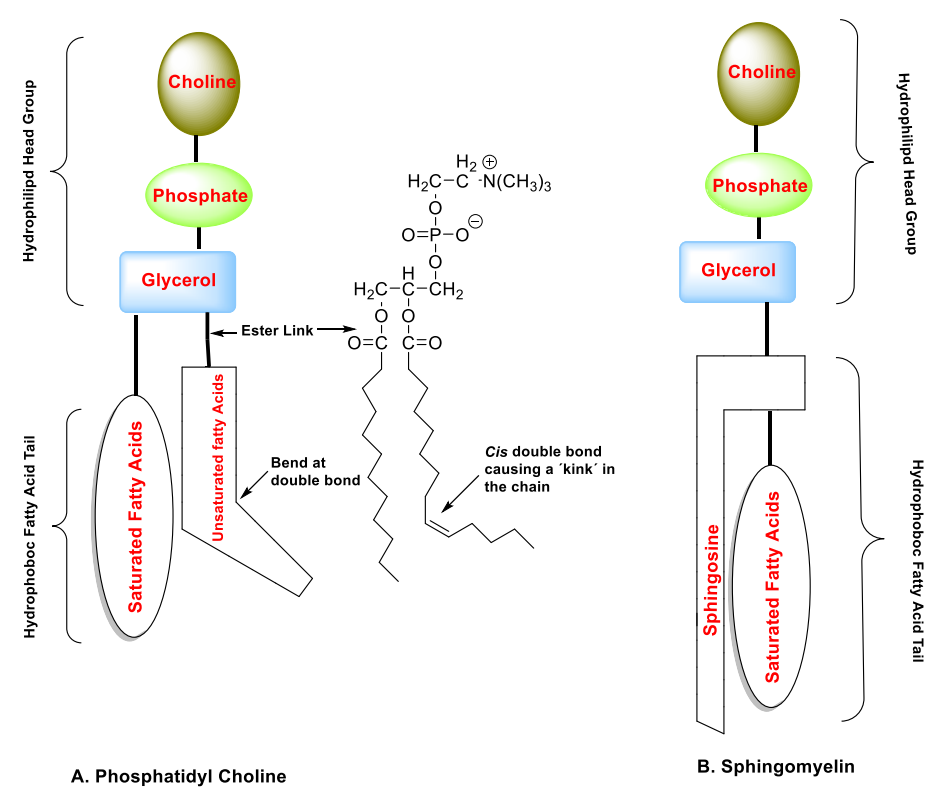

Figure 1.3: Phosphotidyl choline: a glycerophospholipid (A); Sphingomyelin: $a$ sphingophospholipid (B)

\subsubsection{Glycolipids}

Similar to the phospholipids, the glycolipid molecules contain either glycerol or sphingosine linked to the fatty acid chains (Figue 1.4). But unlike the phospholipids the glycolipids contain a sugar, such as glucose or galactose, attached to the glycerol instead of the phosphate containing head group. The glycolipids in animal membranes almost always contain sphingosine, while those in bacterial and plant membranes principally contain glycerol. In general, the glycolipids are found on the outer surface of the plasma membrane with their sugar moieties exposed at the cell surface. 


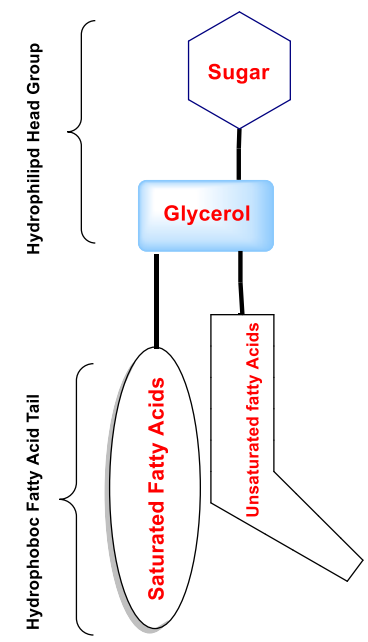

A. A typical glycolipid in animal membrane

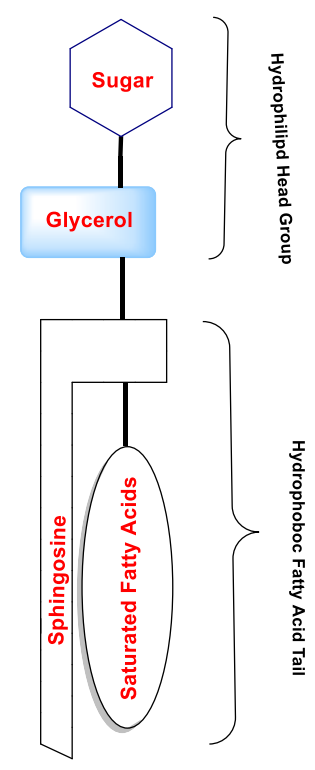

B. A typical glycolipid in bacterial and plant membrane

Figure 1.4: Schematic representation of Typical glycolipids: glycolipid in animal membrane (A) and glycolipid in bacterial and plant membrane (B)

\subsubsection{Cholesterol}

The third type of membrane lipid is cholesterol. It is structurally quite different rom the phospholipids and glycolipids. Cholesterol contain a four-ring steroid structure together with a short hydrocarbon side chain and a hydroxy group (Figure 1.5).

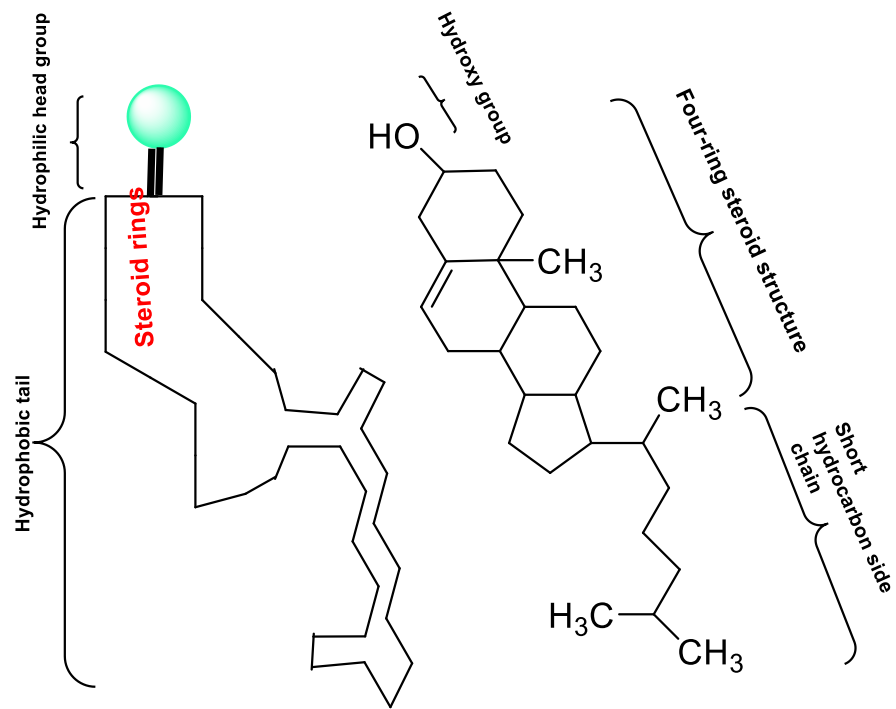

Figure 1.5: Schematic representation of cholesterol 


\subsubsection{Lipid dynamics and flip-flop}

The lipid bilayers are two-dimensional fluids. Membrane lipids and proteins are often observed to move past each other along the membrane (Figure 1.6). In case of phospholipids, this lateral diffusion is very rapid: the diffusion coefficient (the area over which a molecule moves in one second) is approximately $1 \mu \mathrm{m}^{2} \mathrm{~s}^{-1}$. This implicate that a phospholipid molecule is able to diffuse $2 \mu \mathrm{m}$ in $1 \mathrm{~s}$. So, it can move across a bacterial cell in $1 \mathrm{~s}$, or around the perimeter of a human red blood cell in $12 \mathrm{~s}$. Individual lipid molecules also rotate very rapidly along their head-to-tail axes, and the flexible tails can 'wave' at their ends.

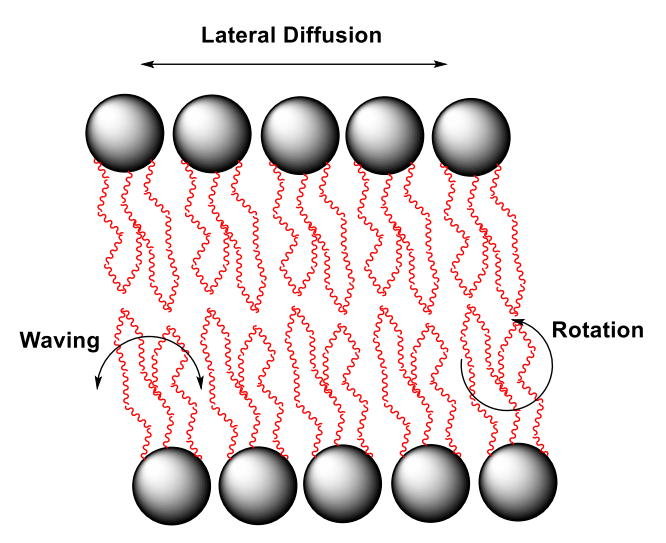

Figure 1.6: Phospholipid dynamics in lipid bilayer

In contrast to the rapid lateral diffusion, lipid molecules rarely move from one monolayer to the other one in the opposite side and often it is found that the composition of the two monolayer vary significantly. The transfer of a phospholipid molecule from one layer to the other is known as transverse diffusion or 'flip-flop'. The dynamics is quite rare as the hydrophilic headgroup has to penetrate the hydrophobic hydrocarbon tail part of the bilayer to reach the monolayer in the opposite direction (Figure 1.7). Thermodynamically this phenomenon is not favourable. Hence, measurement of the flip-flop times of labelled phospholipids in artificial bilayer vesicles exhibit that on average a phospholipid molecule undergoes through the flip-flop dynamics only once in several hours. However, in membranes of the endoplasmic reticulum, where phospholipids are synthesized, a rapid flip-flop of particular lipids occur in presence of a group of proteins known as phospholipid translocators or flippase. 


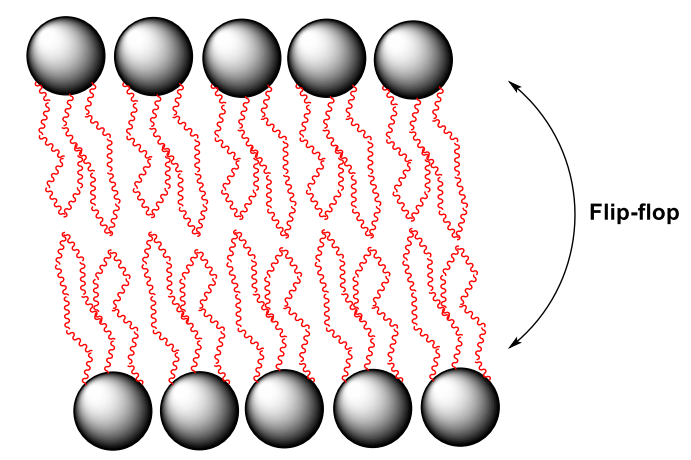

Figure 1.7: Flip-flop dynamics of phospholipids within bilayers

\subsubsection{Membrane fluidity}

Lipids are found in various spatial arrangements and motional freedom by adopting to different fluid and solid phases with respect to the surrounding environment. One of the most important function of the cell membrane is the selective and restricted permeation of substances across the barrier. ${ }^{[5,6]}$ Membrane fluidity plays a key role in regulating the permeability and several other intrinsic properties of the membrane. The fluidity of a lipid bilayer changes primarily due to alteration in fatty acid composition. Besides, temperature and cholesterol content also play a crucial role. [7] At low temperatures, the hydrocarbontails of the bilayer lipids pack closely together to form an ordered arrangement or remain in 'gel phase'. The lipid packing in this form is quite rigid. As the temperature increases, the lipid molecules get more energy to vibrate rapidly causing the bilayer to 'melt' into a more disordered arrangement or 'liquid state', which is more fluid in nature. The exact temperature at which the physical state of a particular lipid bilayer changes from its ordered two-dimensional rigid crystalline form or 'gel phase' to disordered form or 'liquid state', is known as the 'phase transition temperature $\left(T_{m}\right)$ ' for that particular lipid bilayer. The phase transition temperature depends on various parameters, such as, hydrocarbon chain length, temperature and presence of cholesterol in bilayer. [8]

The bilayer fluidity also depends on the fatty acid composition: the transition temperature is lowered or the lipid bilayer becomes more fluid with the shortening of the acyl chain length in the fatty acids as well as with the increment of the unsaturation. The short acyl chains interact less with each other through Van der Waals force in comparison to the longer ones, so a lower temperature is sufficient to change the lipids from the gel phase to liquid phase. ${ }^{[9]}$ On the other hand, the double bonds put bends in the hydrocarbon tails, making it more difficult for the phospholipids to pack together in order to stay in gel phase. Therefore, it requires less energy to disrupt the ordered form of the lipids with bends in the acyl chains to 'melt' to the less ordered 
liquid phase. So, the transition temperature is also lowered due to the presence of double bonds in the fatty acids. [6]

The bilayer fluidity is also influenced by cholesterol. Cholesterol fits in between the phospholipid molecules in bilayer with its hydroxy group close to the hydrophilic phospholipid head group and its hydrophobic rings and side chain buried within the fatty acid chains of the membrane interior. The rigid steroid ring of the cholesterol interact with the neighboring regions of the lipid hydrophobic tails and stiffens them, making the overall membrane less fluid. And this requires higher temperature to 'melt' the lipid from the ordered form to the disordered form and as a consequence the transition temperature is increased due to the presence of more cholesterol in lipid bilayer. ${ }^{[8,9]}$

Therefore, the phospholipids exist in a rigid crystalline gel phase with low membrane fluidity and permeability at a temperature below the critical phase transition temperature $\left(T_{m}\right)$. At a temperature equal to the critical $T_{m}$, the fluidity as well as the permeability of the lipid bilayer increases slightly. In this phase, a highly permeable interfacial area is present between the coexisting gel and liquid phase bilayer domains. At temperature higher than that of $T_{m}$, the fluidity rapidly increases but the permeability of the bilayer does not increase (Figure 1.8). [5]

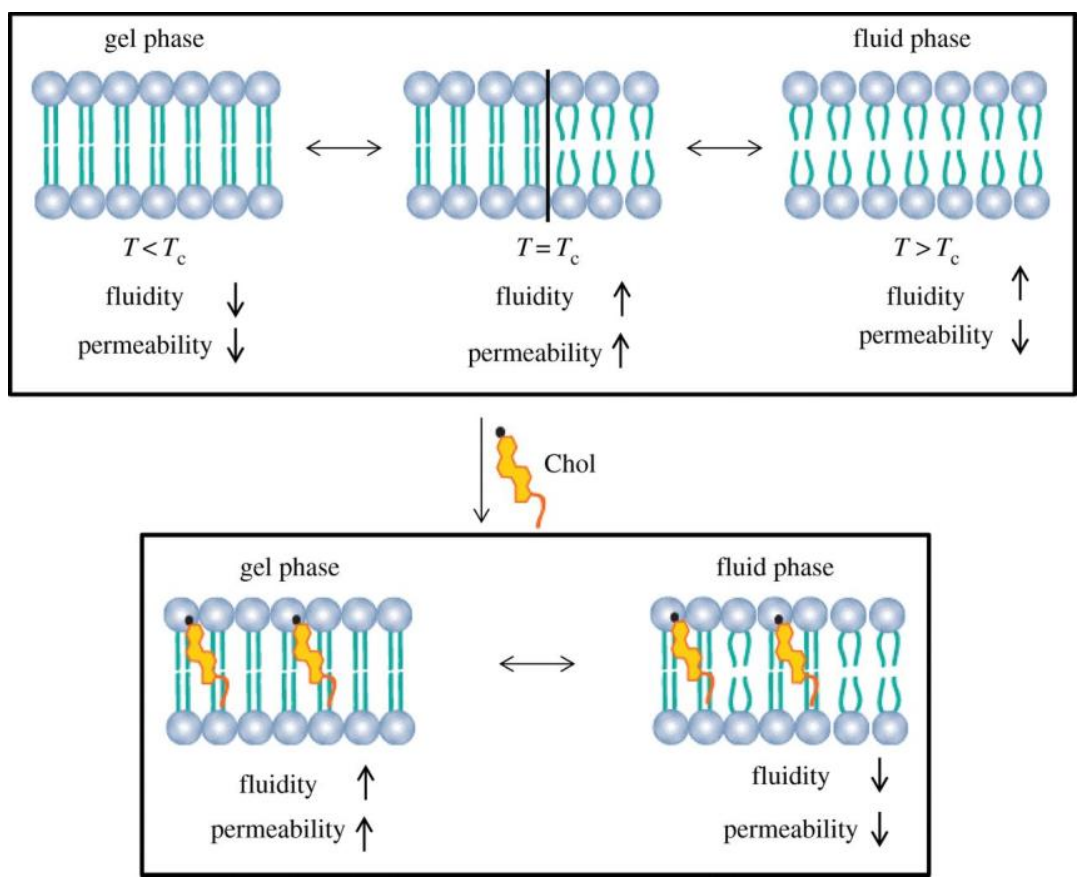

Figure 1.8: Effect of temperature and cholesterol on lipid bilayer fluidity and permeability across phase transition temperature [10] 


\subsection{Membrane Proteins}

Comprising nearly a third of the human genome [11], membraneproteins are a major component of biological membranes and perform a variety of essential cellular functions. [12, 13, 14] Membrane proteins function as enzymes [15, 16, 17], receptors [18, 19, 20] and transport proteins that catalyze the transport of ions $[16,17,21,22]$ and other small molecules for cellular communication. The more active a membrane is in metabolism, it is anticipated that the more proportion of membrane protein it contains. There are various ways in which protein are associated with the lipid bilayers to form functional biological membranes (Figure 1.9).

Transmembrane proteins: Many membrane proteins span across the lipid bilayer. Such transmembrane proteins have hydrophobic regions embedded within the lipid bilayer and interact with the hydrophobic tails of the phospholipids. These regions are often $\alpha$-helical in nature that forms rigid 'tubes' studded with hydrophobic amino acid side chains. There could be one or more such hydrophobic domains in a transmembrane protein. The hydrophilic regions of these proteins protrude from both sides of the membrane. Some transmembrane proteins may also be covalently linked to a fatty acid chain inserted into the phospholipid bilayer (Figure $1.9(\mathrm{~A}))$.

Anchored membrane proteins: Some intracellular proteins do not span the membrane but are covalently attached to the inner surface with a fatty acid chain (Figure 1.9(B)). Such proteins are termed as anchored membrane proteins. They remain firmly attached to the membrane and can only be removed by chemical treatments, like, using detergents or organic solvents that disrupt the membrane. Examples of such type of membrane proteins are the enzyme cholinesterase, which is found in synapses, and the G-proteins involved in sending messages across membranes.

Peripheral membrane proteins: Many membrane proteins are weakly bound to the surface of the lipid bilayer by non-covalent interactions with other membrane proteins (Figure 1.9(C)). These can be removed by mild treatments, such as, alteration of $\mathrm{pH}$ or ionic strength that leave the membrane intact. Such type of membrane proteins are called the peripheral membrane proteins. A very common example of peripheral membrane protein is the cytochrome $c$ located at the inner mitochondrial membrane. 


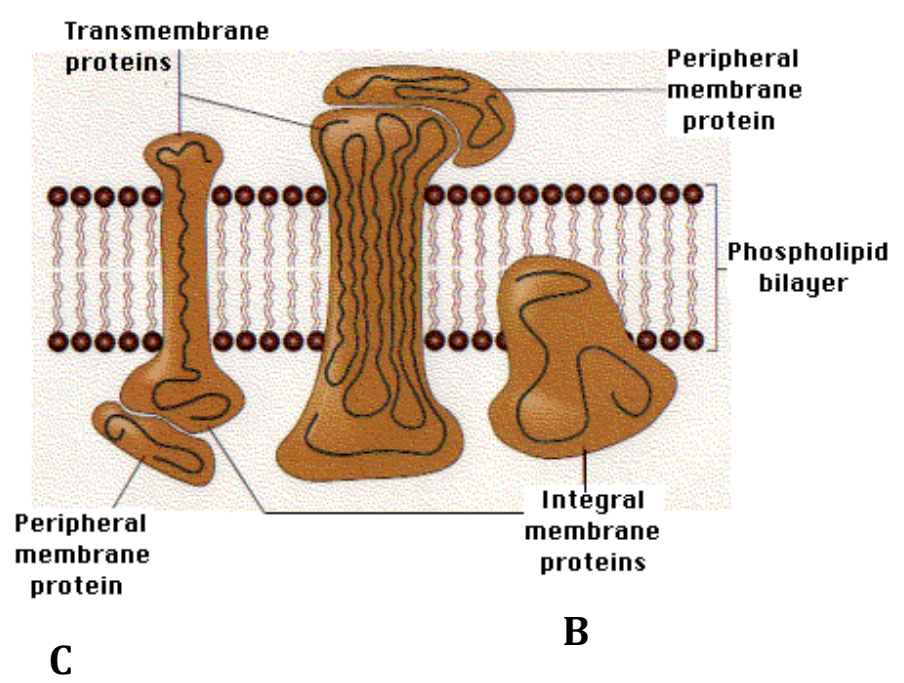

Figure 1.9: Schematic representation of different types of membrane proteins: Transmembrane proteins spanning the bilayer (A); Integral membrane proteins attached to the fatty acid chain of the lipids by covalent interaction (B); Peripheral membrane proteins attached to the transmembrane proteins $(\boldsymbol{C})$

\subsection{Protein-lipid interaction in biological membrane}

Intrinsic membrane proteins contain hydrophobic segments that are in contact with the lipid acyl chains and that are important for stable integration of the protein into the lipid bilayer. From an energetic point of view, it can be expected that the length of the lipid-exposed hydrophobic segments is approximately equal to the hydrophobic bilayer thickness, in order to avoid unfavorable exposure of hydrophobic surfaces to a hydrophilic environment. Yet, proteins that are encountered in one membrane can have different lengths of their hydrophobic parts. On the other hand, membrane proteins with the same length can be encountered in bilayers of different thickness. This hydrophobic mismatch arises from a difference in the hydrophobic thickness of a lipid membrane and a transmembrane protein segment, and is thought to play an important role in the folding, stability and function of membrane proteins.

To investigate systematically complex membrane-protein interactions, simplified model membranes and peptides have been designed. [40-48] The model lipid membranes typically constitute just one or two lipid species, rather than the complex mixture of lipids present in the cell membranes. The model proteins are typically single $\alpha$-helical peptides with a large central hydrophobic region and flanking polar or charged residues. ${ }^{[49-52]}$ As proposed by Killian et. al, the membrane system undergoes different alterations through which it tries to relieve the 
energetic stress developed due to hydrophobic mismatch situation. Various peptides have been designed along the years to mimic the hydrophobic segments of membrane proteins. The orientation of these peptides in mismatched conditions has been explored through a variety of techniques: NMR, X-ray, circular dichroism, Fourier transform infrared spectroscopy, electron spin resonance, and molecular simulations. [23-33] All these studies agree that when the membrane thickness is smaller than the transmembrane segment, the peptide accommodates by tilting. However, the extent of tilt remains controversial, and the experimental measurement of the peptide angle is not straightforward since most techniques required perfectible models as recently shown for NMR. [34-35] The peptide behavior is more uncertain when the hydrophobic bilayer thickness is larger than the hydrophobic peptide length. Several studies suggest that the peptide "snorkels" to some extent, and others hypothesize that the lipid mattress adjusts itself through large deformation around the peptide.[30,36,37] For a very strong mismatch, peptides may not be able to span the membrane anymore ${ }^{[38]}$ and could lie at the hydrophobic-hydrophilic interface. ${ }^{[31,32]}$ In this case, it could be difficult to experimentally separate insertion defects due to protocols of peptide incorporation and mechanistic/energetic impossibility for the peptide to insert correctly within the bilayer. The use of model bilayer composed of surfactants, swollen by hydrophobic solvents, can be advantageously used to successfully insert the peptides before increasing the mismatch. ${ }^{[38,39]}$

\section{Positive hydrophobic mismatch:}

For positive hydrophobic mismatch, i.e., a protein hydrophobic length that is greater than the thickness of the lipid hydrophobic region, one or more of the following adaptations can occur:

1. The $\alpha$-helix can reduce its hydrophobic length by becoming a less tightly bound $\pi$-helix.

2. The peptide can tilt, reducing its exposure to polar groups.

3. The acyl chains near the peptide can order, increasing the local bilayer hydrophobic width.

4. The peptides can oligomerize or aggregate.

5. The peptide can be expelled from the bilayer.

\section{Negative hydrophobic mismatch:}

For negative hydrophobic mismatch, i.e., the protein hydrophobic length is shorter than the thickness of the lipid hydrophobic region, one or more of the following adaptations can occur:

1. The $\alpha$-helix can increase its hydrophobic length by becoming a more tightly bound 310 helix.

2. The bilayer width near the peptide can decrease, by acyl chain disordering. 
3. The peptides may aggregate or oligomerize.

4. The peptide can induce nonlamellar phase formation.

5. The peptide can be expelled from the bilayer.

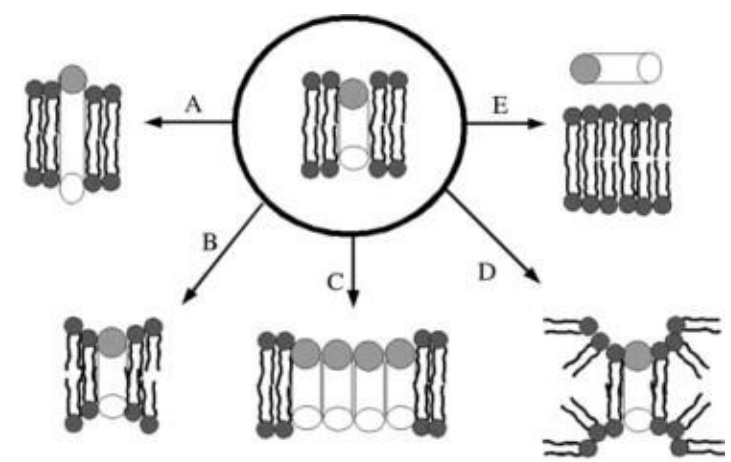

ADAPTATIONS TO NEGATIVE MISMATCH

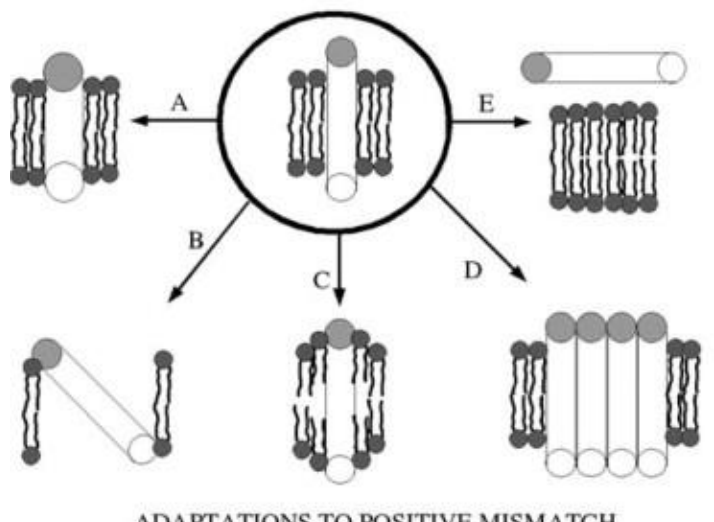

ADAPTATIONS TO POSITIVE MISMATCH

Figure 1.10: Schematic representation of different adaptations for negative and positive hydrophobic mismatch situations (taken from de Planque and Killian) [53]

\subsection{Effect of EtOH in compensating negative hydrophobic mismatch by reduction of bilayer thickness}

The modulation of the properties and function of plasma membranes by small amphiphilic solutes is important for many biomedical applications, including anesthesia, cryopreservation and permeation enhancement. Ethanol, being a short chain alcohol, is a particularly important representative of molecules that can modulate properties of membranes. Apart from the fact that it is widely consumed as a principal component of alcoholic beverages, the ability of ethanol to modulate bilayer thickness can also be an important to tool to investigate extent of negative hydrophobic mismatch compensation via reduction of the bilayer thickness. If a restricted concentration of ethanol can compensate the negative hydrophobic mismatch and help in effective reconstitution of shorter transmembrane peptides into lipid membranes that would 
open up lot of new opportunities in the direction of developing artificial transmembrane domain systems. In this connection, how ethanol interacts with lipid membranes remains the fundamental to each of these applications. This interaction is also of fundamental biophysical interest as ethanol can modulate the phase stability of lipids and can induce the formation of interdigitated bilayer structures.

Given the significance of the interaction of ethanol with lipid membranes, it is not surprising that this area has been the focus of numerous experimental and computational studies. These studies are beginning to reveal a molecular level picture of how ethanol can modulate the structural and mechanical properties of membranes. Although ethanol has an amphiphilic character, being a short-chain alcohol, its hydrophobicity is limited. Therefore, after partitioning into a lipid membrane, ethanol molecules are primarily located within the water/lipid interface forming hydrogen bonds with hydrophilic lipid head groups.[54, 55] Alcohols such as ethanol are amphipathic and can displace some of the water molecules associated with the gel phase lipid bilayer interfacial region, penetrating to some extent into the nonpolar region of the bilayer.11 The displacement of water molecules in the vicinity of the lipid headgroups weakens the interactions between the headgroups, allowing the bilayer structure to dilate, thus increasing the average surface area per headgroup and as well as causing a reduction in the tension at the membrane/water interface and in the membrane rigidity.[58, 59] The addition of a sufficient amount of alcohol can perturb the bilayer interfacial region to the extent that a phase transition to an interdigitated structure occurs. ${ }^{[60-63]}$ Ethanol has been used widely to induce the formation of the interdigitated phase, with much of the early work being done by Rowe et al. [64-66] and Simon et al. $[67,68]$ The interdigitated phase occurs when the acyl chains of lipids from one leaflet interpenetrate with the chains from the opposing leaflet, eliminating the bilayer midplane, and leading to a consequent decrease in bilayer thickness. Hence, The presence of ethanol in the membrane has a disordering effect on lipid hydrocarbon chains, giving rise to an increase in the area per lipid and the overall fluidity of the membrane ${ }^{[56,57]}$ accompanied by a drop in the bilayer thickness (figure 1.11). The decrease in bilayer thickness can have a dramatic effect on the function of proteins imbedded within biological cell membranes as well. [69] 


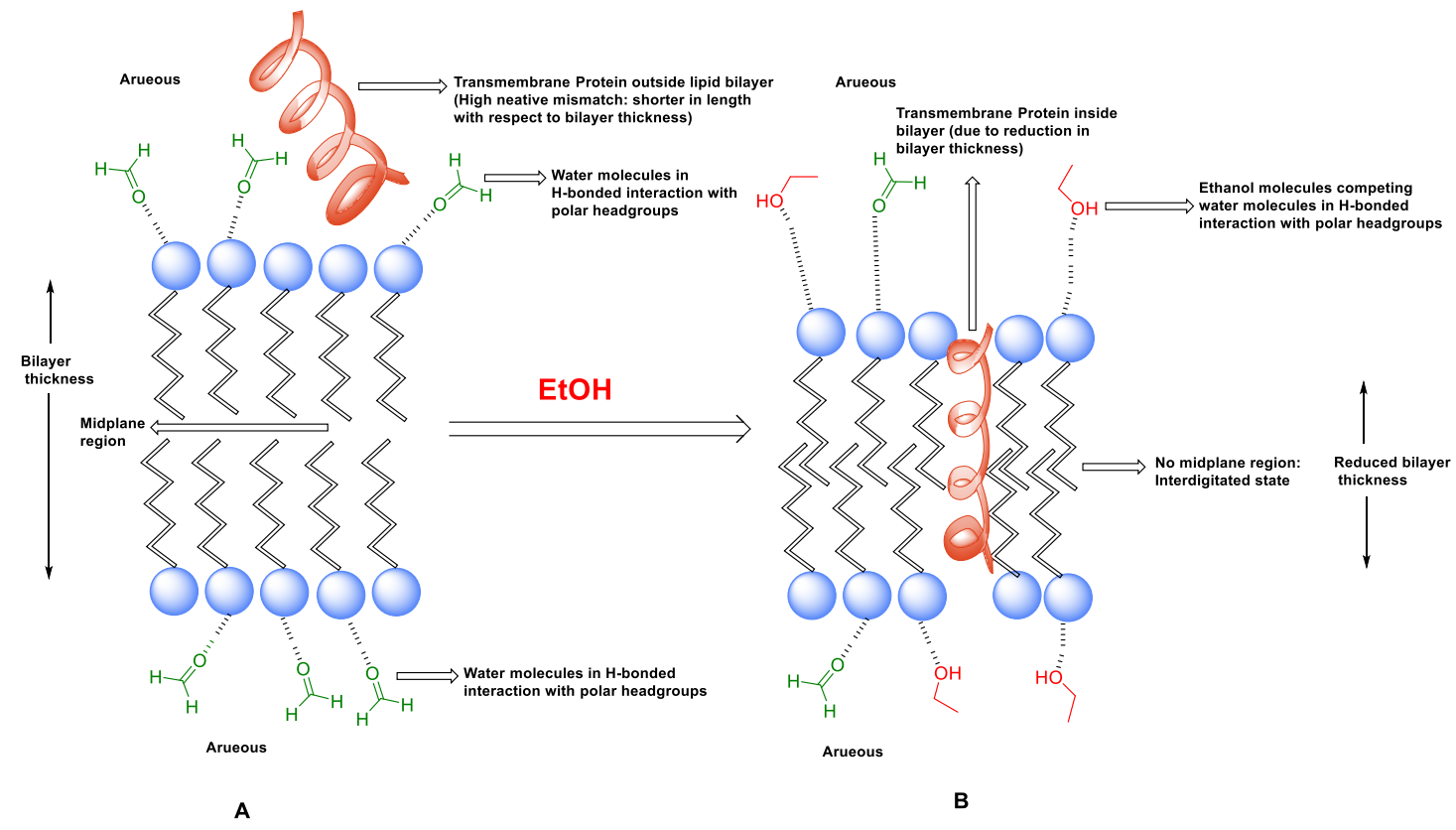

Figure 1.11: Effect of EtOH in compensation of negative hydrophobic mismatch via bilayer thickness reduction; without addition of EtOH (A) and after addition of EtOH (B)

\section{2. $\beta$-Peptide: A model synthetic transmembrane domain}

\subsection{General}

Membrane proteins serve as the functional entities of the biological membrane. All these proteins, responsible for carrying out crucial tasks, starting from enzymatic activities to regulating passage in and out of the cell, are the oligomers of various combinations of different amino acids. Nevertheless, in order to be able to perform those biological functions, the oligomers are found to adopt specific compact conformations that are both kinetically as well as thermodynamically stable. The way of attaining the required compact structure and conformation from the simple oligomeric chain is known as the 'folding'. When these oligomers adopt different characteristic folded structures in order to carry out specific biological functions in cell membrane via 'folding', they are called the 'foldamers', a unique term coined by Gellman. [70] These folding patterns generate "active sites" via precise three-dimensional arrangement of functional groups and these active sites are of immense importance in generating substrate 
specificity in enzyme action. The folded compact structures of proteins imply the tertiary structures. The protein tertiary structures evolves from the specific folding of a regular and conformationally stable secondary structure, such as, the helices, sheets and turns. Therefore, in order to design novel foldamers that efficiently mimic the biological functions, it is extremely necessary to identify new backbones with 'well defined', that is, conformationally stable secondary structural preferences. So, the oligomer with modest length should exhibit strong inclination towards folding into conformationally stable secondary structure in solution. There are a few types of common secondary structures of proteins:

$\underline{\boldsymbol{\alpha}}$-Helices: The most common type of secondary structure in proteins is the $\alpha$-helix. Linus Pauling was the first to predict the existence of $\alpha$-helices. In a helix, the carbonyl $(\mathrm{C}=0)$ of one amino acid is hydrogen bonded to the amino $\mathrm{H}(\mathrm{N}-\mathrm{H})$ of an amino acid that is four down the chain. (E.g., the carbonyl of amino acid 1 would form a hydrogen bond to the $\mathrm{N}-\mathrm{H}$ of amino acid 5.) There are 3.6 residues/turn in a $\alpha$-helix, which means that there is one residue every 100 degrees of rotation (360/3.6). Each residue is translated $1.5 \AA$ along the helix axis, which gives a vertical distance of $5.4 \AA$ between structurally equivalent atoms in a turn (pitch of a turn). This pattern of bonding pulls the polypeptide chain into a helical structure that resembles a curled ribbon, with each turn of the helix containing 3.6 amino acids. The R groups of the amino acids stick outward from $\alpha$ helix, where they are free to interact. In a $\alpha$-helix, the carbonyl oxygen atoms $\mathrm{C}=0$ point in one direction, towards the amide $\mathrm{NH}$ groups 4 residues away $(i, i+4)$ (figure 2.1). Together these groups form a hydrogen bond, one of the main forces in the stabilization of secondary structure in proteins.

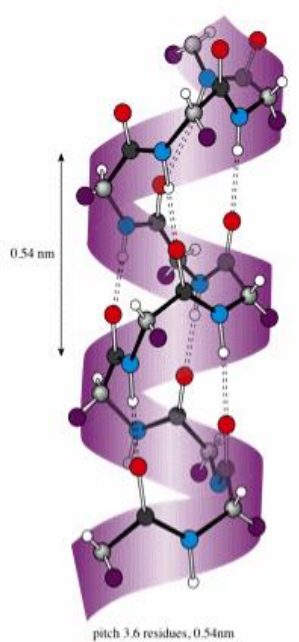

$\alpha$-Helix

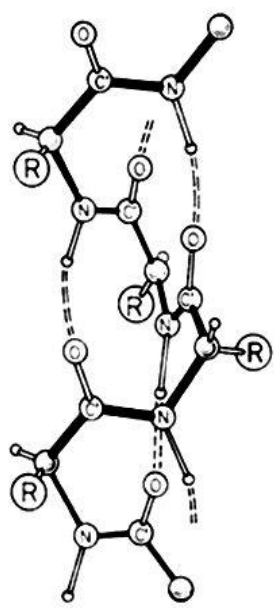

$\alpha$-Heliw

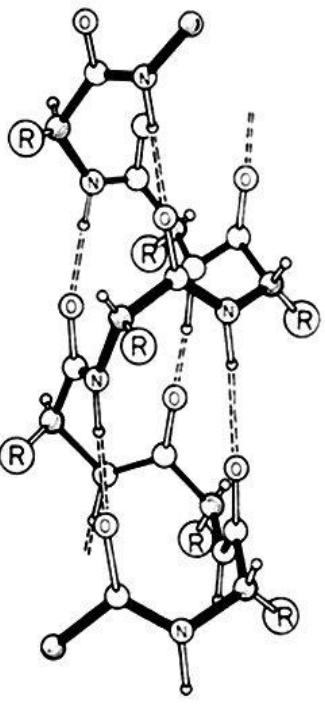

310-Helix

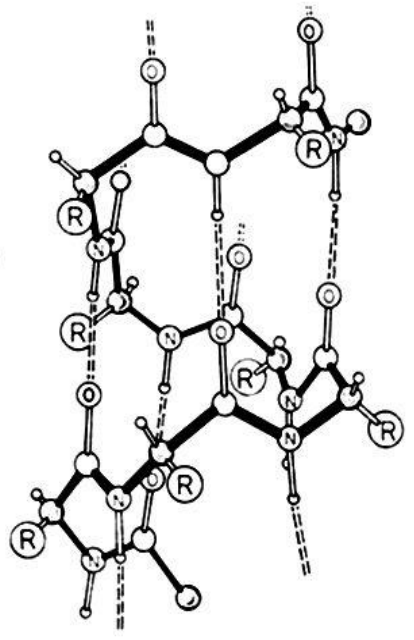

$\pi$-Helix 


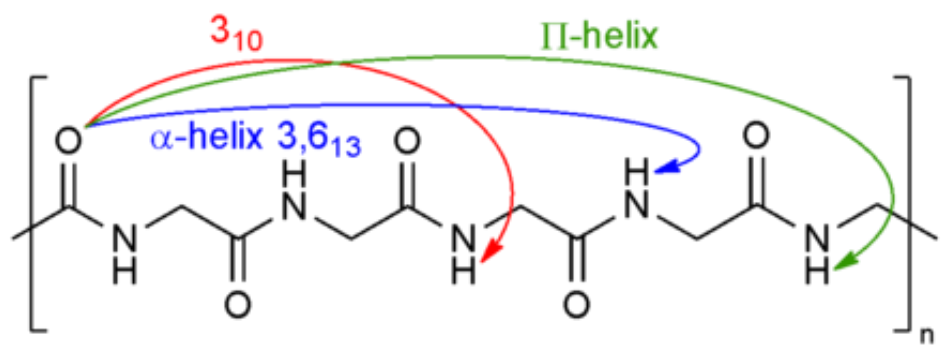

B

Figure 2.1: Schematic representation of $\alpha$-helix, $3_{10}$-helix and $\pi$-helix (A) and hydrogen-bridging in helical protein secondary structures (B)

The $\alpha$-helix is not the only helical structure in proteins. Other helical structures include the $33_{10}$ helix, which is stabilized by hydrogen bonds of the type $(i, i+3)$ and the $\pi$-helix, which is stabilized by hydrogen bonds of the type $(i, i+5)$. The 310 helix has a smaller radius, compared to the $\alpha$-helix, while the $\pi$-helix has a larger radius.

\section{$\beta$-pleated sheet:}

The second major type of secondary structure in proteins is the $\beta$-sheet. In a $\beta$-pleated sheet, two or more segments of a polypeptide chain line up next to each other, forming a sheet-like structure held together by hydrogen bonds. The hydrogen bonds form between carbonyl and amino groups of backbone, while the $\mathrm{R}$ groups extend above and below the plane of the sheet. The strands of a $\beta$-pleated sheet may be parallel, pointing in the same direction (meaning that their $\mathrm{N}$ - and C-termini match up), or antiparallel, pointing in opposite directions (meaning that the N-terminus of one strand is positioned next to the C-terminus of the other).

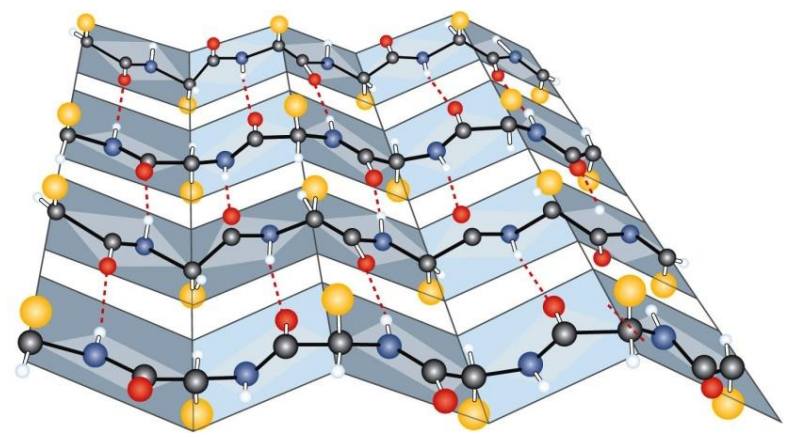




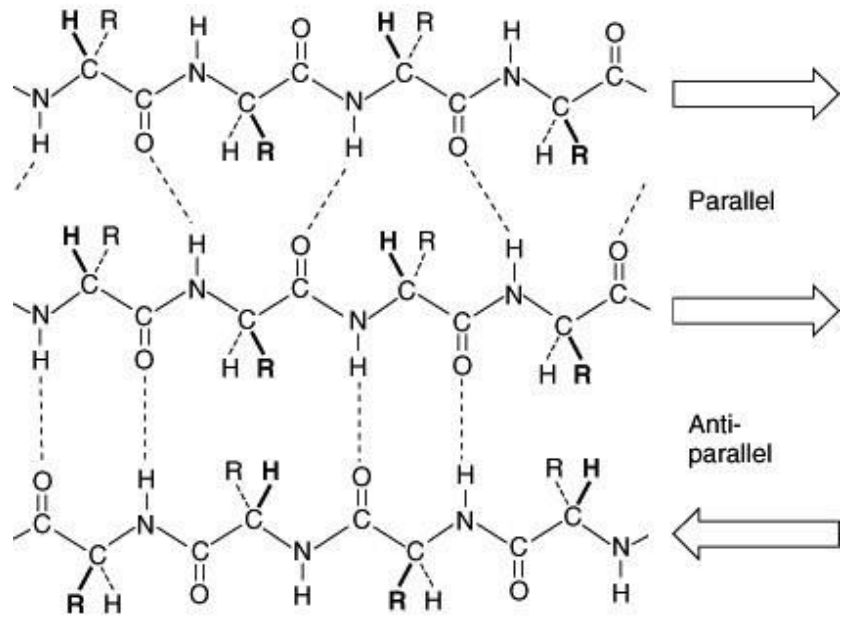

B

Figure 2.2: Schematic representation of $\beta$-pleated sheet secondary structure (A) [71]; parallel and anti-parallel $\beta$-pleated sheet secondary structures (B)

\section{B-Hairpin:}

When there are only two anti-parallel $\beta$-strands, like in the figure below (Figure 2.3), it is called a $\beta$-hairpin. The loop between the two strands is called $\beta$-turn, when it is short. Short turns and longer loops play an important role in protein 3D structures, connecting together strands to strands, strands to $\alpha$-helices, or helices to helices. The amino acid sequences in loop regions are often highly variable within a protein family. Nevertheless, in some cases, when a loop has some specific function, for example interaction with another protein, the sequence may be conserved. Loop length in proteins from organisms living at elevated temperatures (thermophilic organisms) are usually shorter that their mesophilic counterparts, presumably to give a protein additional stability at high temperatures, preventing its denaturation.

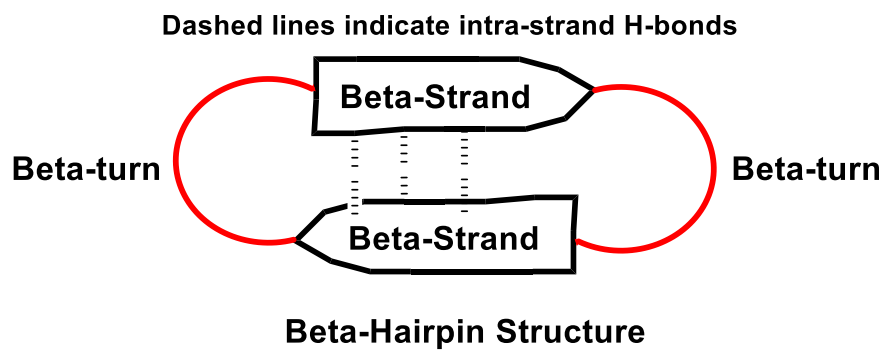

Figure 2.3: Beta-hairpin secondary structures 


\subsection{Evolution of 6 -peptides}

\subsubsection{General}

During the past decades, several oligomers with unnatural backbones that fold into thermodynamically and kinetically stable secondary structures in solution have been explored. Among them, the oligomers of $\beta$-amino acids, i.e., the $\beta$-peptides, mostly took the attention of the scientific community and thus are by far the most well characterized ones. Kovacs et al. first reported the initial model of the helical structure of $\beta$-peptides composed of a poly ( $\beta$-L-aspartic acid) in solution. [72] They proposed that the helical conformation of this $\beta$-polypeptide chain consists of 3.4 residues per turn and an axial translation of $1.58 \AA$ A. Later on, Seebach's group at ETH in Zürich [73] and Gellman's group [74] in University of Wisconsin, USA have been the most active in the exploration of short $\beta$-peptides. [75] In correspondence to the objective of developing model transmembrane domains, it is required to identify backbone that favours $\alpha$-helical secondary structures within moderate oligomer chain length. Therefore, there are two main points of interest in relation to this project to choose $\beta$-peptides as the model for developing artificial transmembrane domain systems:

1. $\beta$-peptides are capable of forming stable $\alpha$-helical secondary structure with as few as four residues.

2. Unlike the $\alpha$-peptide counterparts, the $\beta$-peptides are far more stable towards proteolytic [76, 77] and metabolizing [78] enzymatic degradation. This makes $\beta$-amino acids useful building blocks in peptidomimetic drug design. Several other applications can be also devised by virtue of this unique enzymatic stability of the $\beta$-peptides.

3. $\beta$-peptides are unique to mimic biological functions of $\alpha$-peptidic secondary structures. Significant progress in antibiotic, ${ }^{[79,80,81]}$ anticancer, ${ }^{[82}$ - 85] and anti-HIV functions, ${ }^{[86,87]}$ DNA [88, 89] and RNA [90] binding, and cell penetration. [91] Area with $\beta$ peptidomimetics has already been accomplished. $\beta$-peptides are the oligomers of $\beta$-amino acids. Unlike $\alpha$-amino acids, the $\beta$ amino acids has one extra methylene group $\left(\mathrm{CH}_{2}\right)$ in its backbone. This allows the $\beta$-amino acids to have substituent $(\mathrm{s})$ at both $\mathrm{C}_{\alpha}\left(\mathrm{C}_{2}\right)$ and $\mathrm{C}_{\beta}\left(\mathrm{C}_{3}\right)$ positions and this a $\beta$-amino acid has much higher number of possible derivatives than that of its $\alpha$-amino acid counterparts. A $\beta$-peptide can form various different patterns of hydrogen bonds, which result in various secondary structures. 
<smiles>NC(=O)C(=O)O</smiles>

L- $\alpha-A A$<smiles>[R8]C(N)C(Br)C(=O)O</smiles>

like $\beta^{2,3}-\mathbf{A A}$<smiles>NCCC(=O)O</smiles>

B-hGly<smiles>[R]C(CN)C(=O)O</smiles>

$\beta^{2}-A A$<smiles>NC(Br)CC(O)O</smiles>

$\beta^{3}-A \Lambda$<smiles>[R8]C(N)C([R8])C(=O)O</smiles><smiles>[R]C([R])(CN)C(=O)O</smiles><smiles>[R8]C(N)C([R8])([R5])C(O)O</smiles>

unlike $\beta^{2,3}-\mathrm{AA}$

$\beta^{2,2}-\mathrm{AA}$

$\beta^{2,2,3}-\mathrm{AA}$

Figure 2.4: Schematic representation of various types of $\alpha$-and $\beta$-amino acids [92]

\subsubsection{Major helical secondary structures of $B$-peptides}

According to Balaram et al., the conformations of these secondary structures of the $\beta$-peptides are defined by the torsional angles $\omega, \phi, \theta$ and $\psi$, where the torsional angle of the CO-N-bond is assigned as $\omega$, the $\mathrm{HN}-\mathrm{C}_{\beta}$-bond is designated as $\phi$, and the $\mathrm{CO}-\mathrm{C}_{\alpha}$-bond as $\psi$. The $\mathrm{C}_{\alpha}-\mathrm{C}_{\beta}$-bond describes the most important torsional angle $\theta$ that is one of the most important parameters for secondary structure formation (Figure 2.5 A). ${ }^{[110,134]}$ Besides the torsional angles, the substituent(s) also have considerable effects on determining the local conformation of $\beta$ peptides. The unsubstituted $\beta$-amino acid, $\beta$-alanine, is highly flexible, analogous to glycine in the $\alpha$-amino acids. Alkyl substituent(s) at positions 2 and 3 favor a gauche conformation about the $\mathrm{C}_{2}-\mathrm{C}_{3}$ bond. ${ }^{[93]} \mathrm{C}_{2}, \mathrm{C}_{3}$-Disubstituted amino acids are even more conformationally constrained and favor gauche conformers when the substituent(s) are anti (in aldol convention). Gauche-type torsion angles are even more strongly promoted when these atoms are included in a cyclohexane or cyclopentane ring, as in trans-2-aminocyclohexanecarboxylic acid (ACHC), ${ }^{94,}$ 95] trans-2,5 diaminocyclohexanecarboxylic acid (DCHC),[96, 97] trans-2-aminocyclopentanecarboxylic acid (ACPC),[98, 99] or trans-3- amino-pyrrolidine-4-carboxylic acid (APC).[100] The ring size determines the precise $\mathrm{C}_{2}-\mathrm{C}_{3}$ torsional preference, which in turn influences $\beta$-peptide helix type.[98] 
<smiles>NC1CCCCC1C(=O)O</smiles>

$(R, R)$-trans-ACHC<smiles>NC1CCCC1C(=O)O</smiles>

$(R, R)$-trans-ACPC<smiles>N[C@H]1CC[C@@H](N)C(C(=O)O)C1</smiles>

$(R, R, R)$-trans-DCHC<smiles>NC1CNCC1C(=O)O</smiles>

$(3 \mathrm{~S}, 4 R)$-trans-APC

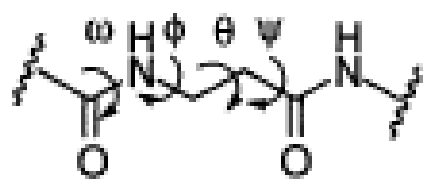

A
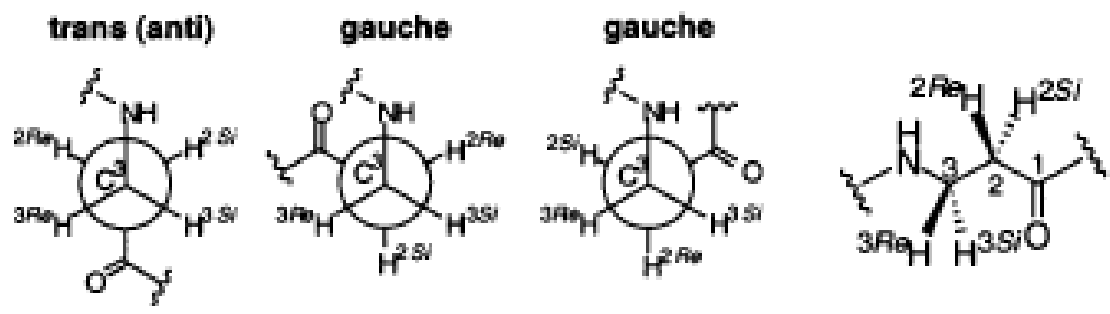

B

Figure 2.5: Schematic representation of torsional angles in $\beta$-peptides $(\boldsymbol{A})$ and different rotamers for $\beta$-alanine in relation to the dihedral angle, $\theta(\boldsymbol{B}){ }^{[101]}$

It is interesting that the $\beta$-peptides are able to mimic the characteristic conformations of the natural $\alpha$-peptides. It is expected that due to an extra methylene group in the backbone, the $\beta$ peptides would possess higher conformational flexibility and with higher degrees of freedom, which in turn would thermodynamically disfavour the formation of ordered conformations. But to utter surprise, the $\beta$-peptides show higher varieties of ordered conformational states. Besides, unlike the $\alpha$-peptide counterparts that require at least $15 \alpha$-amino acids to for a stable $\alpha$-helical secondary structure [102], the $\beta$-peptides exhibit stable $\alpha$-helices with as few as four to six $\beta$ amino acids. ${ }^{[103]}$ As mentioned earlier, a spectrum of different secondary structures arise due to formation of different patterns of hydrogen bonds in $\beta$-peptides. These motifs include different types of helices, parallel sheets and hairpin structures. Since the different helical structures are of greater interest in connection to this project, so each type of hydrogen bonding giving rise to 
different helical secondary structures in $\beta$-peptides is schematically represented in figure 2.6. The types primarily include 14-helix, 12-helix and alternate 10/12-helix, but there are also 10helix and 8-helix present in lower abundance. The naming of the ring is based on the number of atoms in a hydrogen-bonded ring. [101]

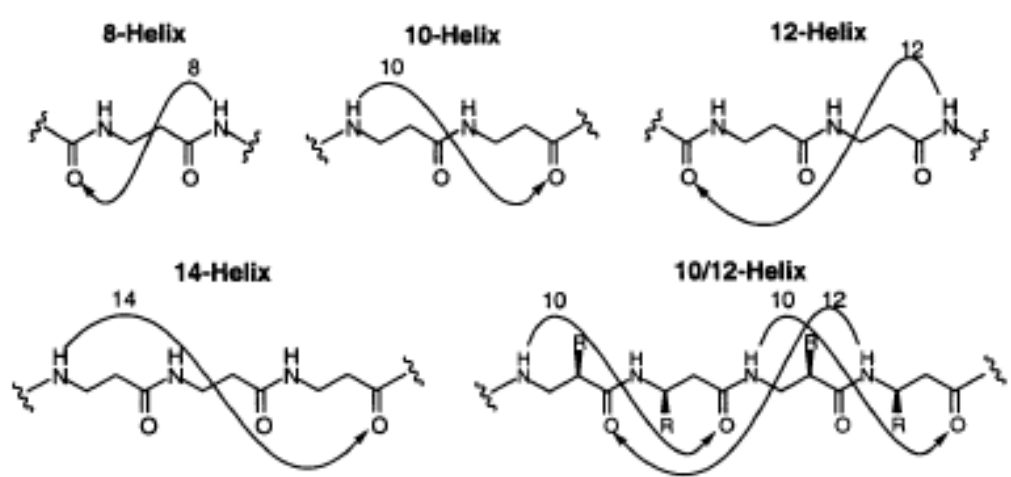

Figure 2.6: Schematic representation of different helical secondary structures of $\beta$-peptides based on different types of hydrogen bonding patterns [101]

\section{4-helix:}

14-helix is formed by contiguous 14-membered ring with hydrogen bonds between the N-H of the $i^{\text {th }}$ residue and the $\mathrm{C}=0$ of $(i+2)^{\text {th }}$ residue along with a three-residue repeating arrangement of the side chains and a pitch of approximately $5.0 \AA \AA^{[104]}$ The unique symmetrical orientation of every third side chain allows an individual assignment of properties of the three helical faces.[105, 106] Therefore, this particular secondary structure is also known as 314 -helix. The overall structure of the 14-helix differs from that of the $\alpha$-helix in many respects. Unlike the $\alpha$-helix that is composed of 13-membered hydrogen-bonded rings with 3.6 amino acids per turn and a radius of $4.3 \AA$, the $33_{14}$-helix has larger radius of $4.8 \AA$, due to the difference in the size of the hydrogenbonded rings [103] The 14-helix repeats approximately every 3 residues, which positions the side chains of every third residue directly atop one another along one face of the helix. The amide carbonyl and $\mathrm{NH}$ groups project toward the $\mathrm{N}$ - and C-terminus, respectively resulting in a net dipole opposite to that of the $\alpha$-helix. [101] Furthermore, Gung et al. and Hamuro et al. have previously proposed that the presence of valine side chains enhance the formation of the $34^{-}$ helix of $\beta$-peptides. $[107,108]$ Apart from that, as discussed before cyclic groups like, trans-2aminocyclohexanecarboxlyic acid (achc) also favours the formation of the 314-helical 
structures. ${ }^{[109]}$ Considering these conformational advantages, the $3{ }_{14}$-helical peptides have been extensively explored in the field of peptidomimetics.

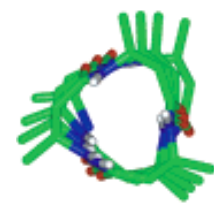

A

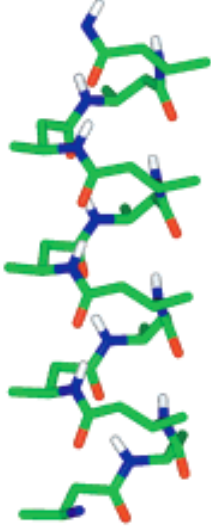

B

Figure 2.7: Structure (Top view (A) and side view (B)) of a typical 14-helix (the hydrogens are omitted for better clarity except the amide hydrogen (white). Carbon atoms are depicted green, nitrogen in blue and oxygen in red)

\section{2-helix:}

The prediction that oligomers of ACPC favouring the formation of a new helical secondary structure, the 12-helix, evolved from the experimental observations, $[\mathbf{9 8 , 1 0 0 , 1 1 0 ]}$ in which relatively short oligomers were shown to adopt the 12-helix conformation, both in organic solution and in the solid state. In organic solvents, the conformation is so stable that peptides with as few as six ACPC residues forms a stable 12-helix. Unlike, cyclohexyl group like, ACHC stabilizing the torsional angle $(\theta)$ to a value about $\pm 60^{\circ}$, the cyclopentyl ring, ACPC, stabilizes the torsional angle $(\theta)$ to a higher value, which in turn gives rise to a unique helical secondary structure, the 12-helix. The 12-helix is stabilized by hydrogen bonds between amides carbonyl groups at position $i$ and an amide proton at position $i+3$ in sequence. The helix repeats approximately every 2.5 residues and shows the same polarity as the $\alpha$-helix. 


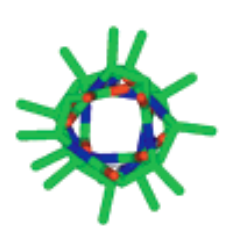

A

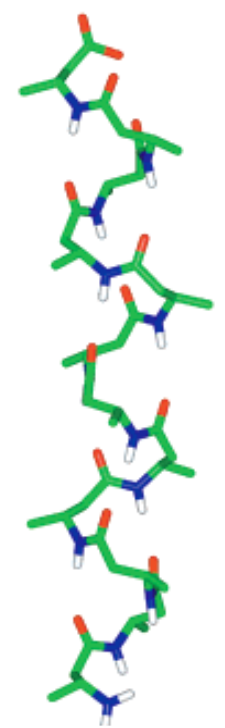

B

Figure 2.8: Structure (Top view (A) and side view (B)) of a typical 12-helix (the hydrogens are omitted for better clarity except the amide hydrogen (white). Carbon atoms are depicted green, nitrogen in blue and oxygen in red)

\section{0/12-helix:}

$\beta$-Peptides with alternating $\beta^{2}$ - and $\beta^{3}$-monosubstituted residues can adopt the alternate 10/12helix conformation. [93,111] The characteristic feature of this helix is an intertwined network of alternating 10- and 12- member hydrogen-bonded rings. In this helix, amides surrounded by methylene hydrogen bond to one another $(i, i+2)$, forming the 10 -membered rings, while the 12 atom rings are formed between amides surrounded by side chains $(i+1, i+3) .[111]$ In contrast to the uniform alignment of amide bonds with the helical axis for the 14- and 12-helices, there are two types of amide bond orientations in the 10/12-helix. The 10-atom ring amides are approximately perpendicular to the helical axis, while the 12-atom ring amides are nearly aligned parallel to the helical axis. These results in overall nullified helix dipole moment compared to the other helical conformations. Therefore, this makes the 10/12-helix a unique secondary helical structure with zero helical dipole moment. It has long been theoretically proposed by Killian et. al. [112] and Pablo [113] et. al. that the helical dipole moment might be a crucial parameter to regulate the transmembrane insertion and self-association process of transmembrane peptide domains, respectively. However, due to the synthetic challenges for accessing such type of transmembrane $\beta$-peptide segments, the actual function of the helical dipole moment in membrane insertion has not yet been studied experimentally. On the other 
hand, it is speculated that the 10/12-helix is strongly favoured when the residues with interacting side chains are placed three residues apart. $\left[{ }^{93,111]}\right.$ It is quite surprising to observe that considering only the backbone of the 10/12-helix, it is intrinsically much more stable than that of the 14- or 12-helical secondary structures. Due to the unique and surprising nature of the 10/12-helix, it is the focus of this thesis to shed light on the effect of helical dipole moment in membrane insertion of $\beta$-peptide based artificial model transmembrane domains. For further detailed studies, it is essential to understand what the helical macrodipole moment is and what its significance is.

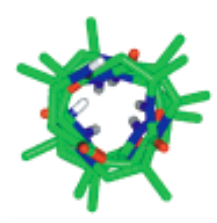

A

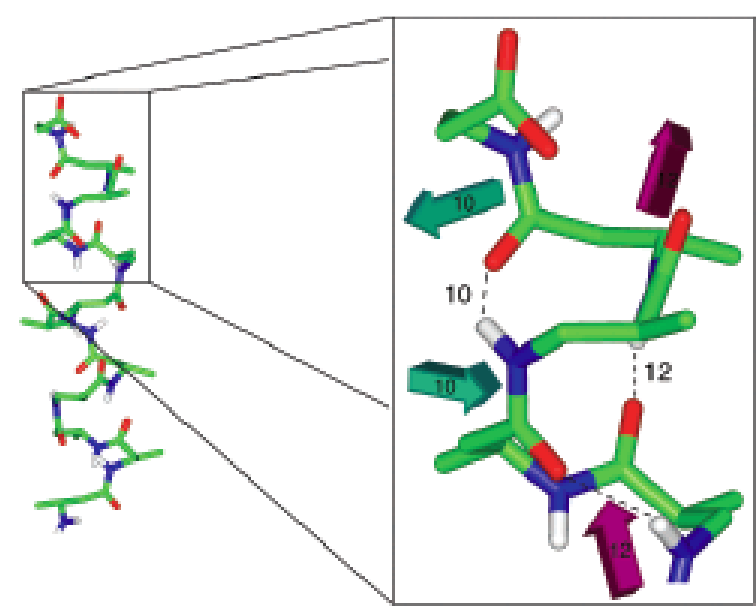

B

Figure 2.9: Structure (Top view (A) and side view (B)) of a typical alternate 10/12-helix explaining the nullification of overall helical macrodiploe moment (the hydrogens are omitted for better clarity except the amide hydrogen (white). Carbon atoms are depicted green, nitrogen in blue and oxygen in red)
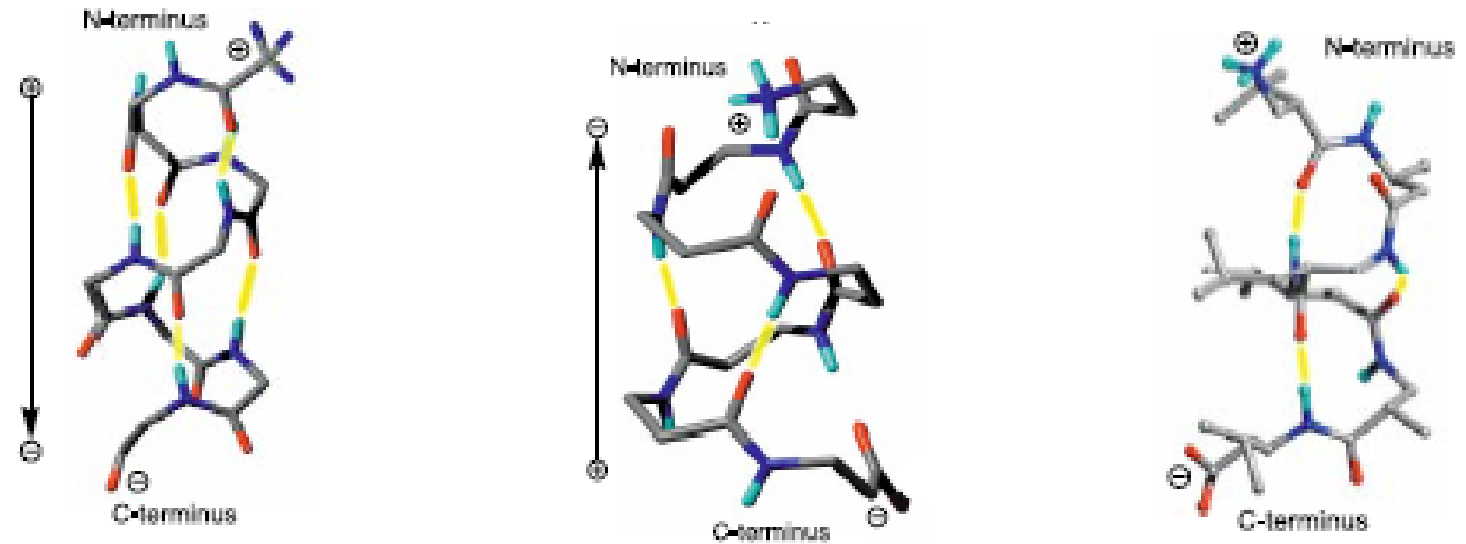

Nullified Zero macro-dipole moment 


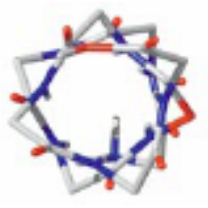

A

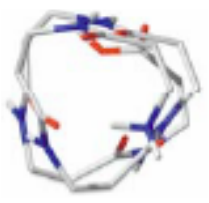

B

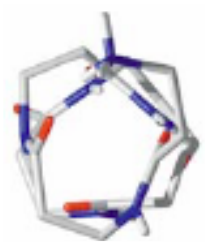

C

Figure 2.10: Comparative schematic representation of $\alpha$-helix with a macro-dipole moment from $N$-to-C terminus (A); a 14-helix with an overall macro-dipole moment from $C$-to- $N$ terminus, i.e., opposite to that of $\alpha$-helix (B); an alternate 10/12-helix with overall nullified zero macro-dipole moment $(\boldsymbol{C})$

\begin{tabular}{|c|c|c|c|}
\hline Secondary Structure & Rise $(\AA)$ & Pitch $(\AA)$ & Residues/turn \\
\hline$\alpha$-helix ${ }^{[18]}$ & 1.5 & 5.4 & 3.6 \\
\hline 14-helix $^{[99]}$ & 1.7 & 5.2 & 3.1 \\
\hline 12-helix $^{[119]}$ & 2.2 & 5.9 & 2.7 \\
\hline $10 / 12-$ helix $^{[92]}$ & 2.1 & 5.7 & 2.7 \\
\hline
\end{tabular}

Table: 1: At-a-glance geometrical parameters for different types of $\beta$-peptides

\subsubsection{Peptide helical macro-dipole moment}

Throughout the scientific literature on protein research of the last thirty years, the $\alpha$-helix dipole is mentioned in a number of cases where its partial charge on both the C-terminal and the $\mathrm{N}$ terminal ends is thought to be involved in various biological processes on a molecular level. In order to understand the cause of this dipole moment, one must look at the structure and the geometry of the $\alpha$-helix. The $\alpha$-helix is a right-handed coiled structure. Each amino acid makes a turn of about 100 degrees and hence it requires 3.6 residues to make a full turn. Amino acids that wind around the axis form hydrogen bounds with each other. The N-H group of each amino acid forms a hydrogen bond with the $\mathrm{C}=\mathrm{O}$ of the amino acid that is located four places earlier in the helix. The vast number of hydrogen bonds that is formed is actually the underlying reason for the forming of this secondary protein structure. Although the principles behind the occurrence of the helix dipole have been described earlier, Hol first reviewed its role in protein structure and function in $1985^{[114]}$. He states that the helix dipole originates in the dipole of the individual peptide unit. The charge distribution within such a unit is depicted in Figure 2.11. Its direction 
is parallel to the $\mathrm{N}-\mathrm{H}$ and $\mathrm{C}=\mathrm{O}$ bonds. It has been shown that in a $\alpha$-helix around $97 \%$ of all peptide dipole moments point in the direction of the helix axis, the dipole is therefore quite insensitive to the $\varphi$ and $\psi$ angles. The $\mathrm{C}=0$ groups are in a slightly upward direction (toward the C-terminus) and the N-H groups are in a downward direction (toward the N-terminus) and this gives rise to a small dipole. The aggregate effect of all individual dipoles in a $\alpha$-helix gives rise to a net negative dipole moment at the C-terminal end, and a positive dipole moment at the $\mathrm{N}$ terminal end of the helix. The dipole moment of an individual peptide unit is about 3.46 Debye (D) which equals 0.72 e $\AA$ or 0.5 e per $1.5 \AA$. Since the axial shift per residue in a $\alpha$-helix is also $1.5 \AA$, all dipoles cancel out except for the $\mathrm{C}$ - and N-terminal ones.

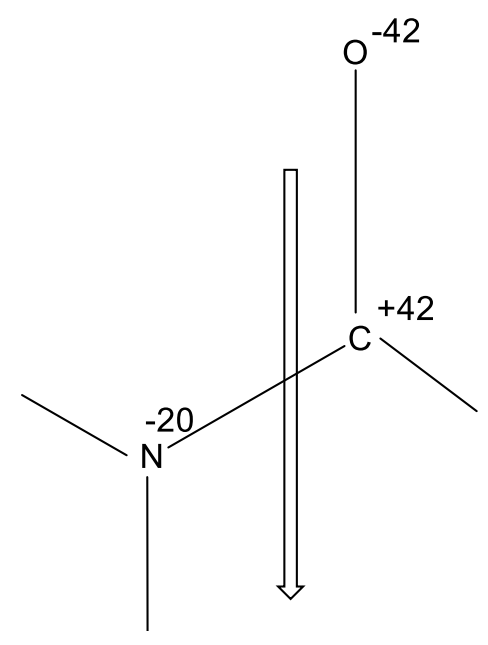

$\boldsymbol{\mu}$

Figure 2.11: Excess charge distribution in a peptide unit showing the resultant dipole moment $(\boldsymbol{\mu})$ [114]

Hence, the origin of helical dipole moment in $\alpha$-peptides has long been explored. However, unlike the $\alpha$-peptides, the same for the $\beta$-peptides still lack in experimental investigations. Synthetic $\beta$-peptides represent a particularly intriguing class of molecules for fundamental studies in the area of peptidomimetics. Being the oligomers of $\beta$-amino acids, $\beta$-peptides offer considerable control over the stability of the secondary structure. The $\beta$-peptides are observed to fold into various helical secondary structures among which the 14-helical and 12-helical structures are more commonly found. The nomenclature of these helices is based on the number of atoms in the characteristic hydrogen bonds. For example, a $\beta$-peptide 14 -helix is defined by $i$, $i-2 \mathrm{C}=0 \cdots \mathrm{H}-\mathrm{N}$ hydrogen bonds that contain 14 atoms and 12 helix by $i, i+3 \mathrm{C}=\mathrm{O} \cdots \mathrm{H}-\mathrm{N}$ hydrogen bonds containing 12 atoms. In both these helices, just like the $\alpha$-peptides, all the individual dipoles cancel out and remains of the ones at the $\mathrm{C}$ - and $\mathrm{N}$-terminals. The amide bonds are uniformly aligned parallel to the helical axis and this gives rise to an overall net macrodipole 
moment in 14- and 12-helices. Nevertheless, unlike the $\alpha$-peptides, the net helical macrodipole moments for these two $\beta$-peptide helices are from $\mathrm{C}$ - to $\mathrm{N}$-terminal. Therefore, these two commonly found $\beta$-peptide helices exhibit an inverted overall helical macrodipole moment. On the other hand, a third type of helical secondary structure is also found that is composed of alternating 10- and 12-membered hydrogen bonded rings yielding an alternate 10/12-helical secondary structure. The 10 -membered ring is defined by $i, i-1 \mathrm{C}=0 \cdots \mathrm{H}-\mathrm{N}$ and the 12 -membered ring by $i, i+3 \mathrm{C}=0 \cdots \mathrm{H}-\mathrm{N}$. In 12 -membered hydrogen bonded ring, the amides are aligned parallel to the helical axis (just as the 12-helical secondary structure). However, in case of the 10membered ring, unlike all the other types of helices, the amides are aligned almost perpendicular to the helical axis. Therefore, the alternate parallel and perpendicular orientation of the amides in 10/12-helix ultimately nullifies the helical dipole moments and gives rise to a unique peptide secondary structure with zero macrodipole moment. This unique feature of the alternate 10/12helical secondary structure of the $\beta$-peptides with no macrodipole moment draws scientific interest in the field of verification of any effect of helical macrodipole moment in different physico-chemical properties the peptides. The experimental knowledge in this field would shed light and contribute significantly in various aspects of peptidomimetics. As for example, it has long been reviewed by Hol et. al. that one in four enzymes of known conformation has an $\alpha$ helical macrodipole moment that affects the electric field in its active sites. ${ }^{[114]}$ Hol et. al. also investigated elaborately the anion-binding preferences of $\alpha$-helices depending on their helical macrodipole moments. However, unlike the $\alpha$-peptides, accurate experimental estimation of helical marodipole moments for any of the secondary helical structures of the $\beta$-peptides is still yet to be done. Although there have a several postulates and theoretical studies based on molecular dynamic simulations published indicating the possible role of the helical macrodipole moment of the $\beta$-peptides in regulation of various physico-chemical and biochemical properties including, dipole induced self-assembly[113], intra-helical aggregation[115], insertion into lipidmembranes[112], voltage-gating in relation of ion-channel formation,[116, 117] but there is hardly any experimental report on the effect of helical macrodipole moment in regulating these properties. The prime reason behind this unavailability of experimental investigations is the synthesis of the zero-dipole $\beta$-peptides with alternating 10/12-helical secondary structure. It is necessary to experimentally access the $10 / 12$-helical $\beta$-peptides with zero macrodipole moment and compare the results of physico-chemical or biochemical experiments with that of the other types of $\beta$-peptides, such as, 14-helical, that have a net macrodipole moment. Only after the efficient comparison, it is possible to shed light on any possible effect of helical macrodipole moment on the mentioned physico-chemical or biochemical properties experimentally. So, it is the main objective of this project to successfully design and synthesize synthetic $\beta$-peptides with 
nullified helical macrodipole moment and shed light on the effect of the latter on the lipidmembrane insertion. Since, the project mainly deals with the development of $\beta$-peptide based artificial transmembrane domain systems, the fundamental knowledge on the effect of helical macrodipole moment on the membrane insertion is of the greater interests.

\section{3. $\beta$-Peptide: Design}

\subsection{General}

Integral membrane proteins are involved in a wide range of important biological processes including the transport of molecules across the membrane, signal transduction, catalysis of reactions, and membrane shaping and stabilization. A detailed investigation on the functioning of integral membrane proteins and molecules modifying their physiological functions is of great interest: not only since integral membrane proteins represent between 20 and $30 \%$ of the proteome of most organisms, $[\mathbf{1 2 0 , 1 2 1 ]}$ but also due to their importance as pharmaceutical targets: approximately $60 \%$ of all drugs target membrane proteins of which integral membrane proteins constitute the largest group.[122] To overcome the shortcomings of the natural $\alpha$-peptides, the extensive exploration of the chemistry of $\beta$-peptides emerged in the past decade. Perhaps, the more promising motivations behind the evolution of $\beta$-peptides in the field of peptidomimetics have been:

(i) That $\beta$-peptides fold in a predictable way to form various types of stable helical secondary structures in solution with short chain lengths;

(ii) They are stable to cleavage by peptidases and to metabolic transformations; and

(iii) They can mimic $\alpha$-peptides in protein-protein and protein-lipid interactions. Therefore, it has been one of the most crucial aspects to investigate the typical structural characteristics of membrane-spanning $\alpha$-helices to design $\beta$-peptide based artificial model transmembrane domain systems.

\subsection{Structural characteristics of transmembrane $\alpha$-helices in membrane spanning and at membrane-water interface region}


Membrane-spanning $\alpha$-helices are composed of a stretch of predominantly hydrophobic amino acid residues, so that the hydrophobic face of these $\alpha$-helices and the acyl chains of membrane lipids can interact with each other by Van der Waals force of interactions in an energetically efficient manner. The principle of hydrophobic matching, in which the hydrophobic length of a transmembrane protein segment matches the hydrophobic thickness of the lipid bilayer, was introduced by Mouritsen and Bloom [123] and is believed to be an important determinant in protein-lipid interactions. The $\alpha$-helix is favorable because this structure satisfies the hydrogen bonding potential of the polypeptide backbone. An alternate structure that lacks a single hydrogen bond would be less stable by about $5 \mathrm{kcal} / \mathrm{mol}$ [124], and therefore, a continuous helix without turns is most favorable. Typical transmembrane $\alpha$-helices are estimated to vary in length from 15 to 28 residues ${ }^{[125]}$ and may be tilted with respect to the bilayer normal, as can be deduced from available three-dimensional structures of membrane proteins.

Compared to the hydrophobic core of the membrane, the membrane-water interfacial (phospholipid headgroup) region presents a chemically complex environment, which offers many possibilities for non-covalent interactions with protein side chains [126]. Therefore, the interface is thought to play an important role in membrane association of proteins and peptides. The carbonyl moieties of the lipids, the phospholipid headgroups and water molecules around the lipid head groups present opportunities for dipole-dipole interactions and allow hydrogen bonding with appropriate amino acid side chains. In addition, electrostatic interactions might occur between, for example, positively charged amino acid side chains and negatively charged lipid phosphate groups. Residues that flank the hydrophobic membrane-spanning segments of membrane proteins will interact with the interface on each side of the membrane and thereby may determine the precise interfacial positioning of these segments or influence their orientation in the membrane [125, 127-130]

A remarkable feature that pops up in many transmembrane $\alpha$-helices is the distribution of aromatic amino acid residues. Not only do membrane proteins have a significantly higher tryptophan content than soluble proteins [131], they are found with a high frequency near the ends of transmembrane $\alpha$-helices, which are expected to be located in the membrane interfacial region. [132] Localization of aromatic amino acids in the membrane interface is for instance observed in the photosynthetic reaction center [133], the potassium channel KcsA [134], bacterial porins [135], and in gramicidin A [136]. In addition, several studies on smaller membraneassociated peptides have shown that there are indeed specific interactions of aromatic amino acid residues with the membrane interface [137-139]. A variety of roles have been proposed for 
interfacial aromatic residues, including positioning or anchoring the transmembrane segments in the membrane and stabilization of the helix with respect to the membrane environment [139].

Another feature that is common in transmembrane proteins is the occurrence of charged residues such as arginine and lysine next to the transmembrane segments, and therefore acting as 'flanking residues'. Although the role of these charged residues in membrane proteins as topological determinants is well-established [140], they may also play a role in membrane anchoring in the interfacial region [139].

So, based on these investigations, the peptide sequence of Gramicidin A, a polypeptide antibiotic that forms single ion monovalent cation channels in biological membranes, was chosen as a model to design $\beta$-peptide based artificial transmembrane domain systems. The linear gramicidin $\mathrm{A}$ is a prototypical channel former that have been extensively used to study organization, dynamics and function of membrane-spanning channels. [141, 142] It is a linear pentadecapeptide antibiotic with a molecular weight of $\sim 1900$. Gramicidin A is produced by the soil bacterium Bacillus brevis, and uniquely consists of alternating L- and D-amino acids. [143] They form well-defined cation-selective ion channels in model membranes [144] with conductance of the order of $\sim 107$ ions per second. Due to their small size, ready availability and the relative ease with which chemical modifications can be performed, Gramicidin A serves as an excellent model for transmembrane channels. Therefore, it has been a logical approach to consider the linear hydrophobic peptide sequence of Gramicidin A as the basis for designing $\beta$ peptide based synthetic transmembrane domain systems in order to address the shortcomings of $\alpha$-peptide based transmembrane domain systems via peptidomimetic strategy.

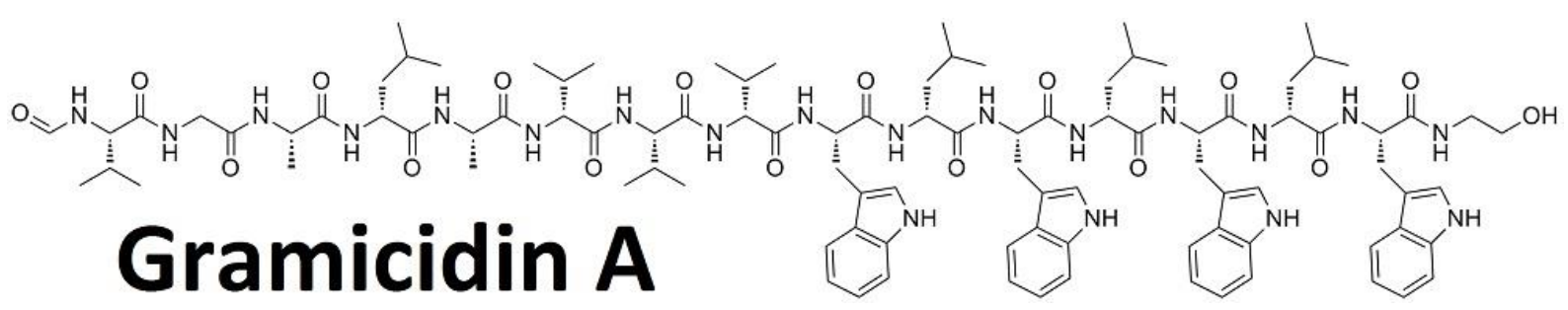

\section{Figure 3.1: Peptide sequence of Gramicidin A}

So, the apolar amino acid triad, Ala/Leu/Val, is chosen as the hydrophobic core sequence for the model $\beta$-peptide based synthetic transmembrane domain system. A tryptophan is thought be incorporated towards the C-terminal of the sequence mainly to verify the location of the transmembrane sequence in solution or in lipid bilayer by detecting the tryptophan 
fluorescence. It is also planned to add at least one lysines on each C-and N-terminals of the sequence, so that the polar residues could favourably interact with polar lipid headgroups in the membrane-water interface region. Since, the effect of the tryptophan towards anchoring the peptide sequence to the membrane is still debated, so addition of extra tryptophans for this purpose is initially excluded to make the design as simple as possible keeping extensive synthetic challenges in mind.

Although the L-enantiomers of amino acids are mostly found in nature and in natural proteins are composed of L-amino acids. Nevertheless, repeated studies have exhibited that the unnatural D-amino acid counterparts might have serious advantages in synthetic peptide design. For example, proteins or peptides composed of D-amino acids are often observed to have higher enzymatic stability and are less prone to enzymatic degradation in presence different proteases. That is exactly what one of the most important reasons for choosing $\beta$-peptides to design synthetic transmembrane domains as $\beta$-peptides are also reported to exhibit stability in presence of protease enzymes. Therefore, the idea is if D-enantiomers of $\beta$-amino acids are used to design the $\beta$-peptide-based transmembrane domains that might prove to be extremely advantageous towards added enzymatic stability. So, D-enantiomers of the selected $\beta$-amino acids are used to access the designed transmembrane domain system. On the other hand, it has also been observed that $\mathrm{D}$-amino acids have the ability to form more stable secondary structures with less number of residues. Hence, this also perfectly coincides with the reason why the $\beta$ amino acids are chosen instead of the $\alpha$-amino acid counterparts.

It has been reported that alpha helices are better preserved in homochiral, rather than heterochiral, peptide sequences. ${ }^{[145]}$ Therefore, the alternating D/L chirality of Gramicidin A is dropped and instead a homochiral D- $\beta$-amino acid repetitions are considered for the design of $\beta$ peptide based artificial transmembrane domain systems. The sequence length is also kept short between 10-12 amino acid. There are two main reasons behind keeping shorter sequence;

i) The shorter the sequence length of the $\beta$-peptides with helical dipole moment less is the magnitude of their helical macrodipole moment as the overall macrodipole moment is the sum of the individual dipole moments of the amino acids. Therefore, it would explain whether minimal difference in the value of helical dipole moment could exhibit any significant change in the physico-chemical or biochemical behaviors of the transmembrane $\beta$-peptides. 
Ii) Evident from the previous literature, there is considerable challenges to synthesize such types of hydrophobic $\beta$-peptides. Therefore, keeping it as short as possible would increase the chance of getting successful.

Hence, the general structure of the designed $\beta$-peptide based artificial transmembrane sequence is depicted in Figure 3.2.

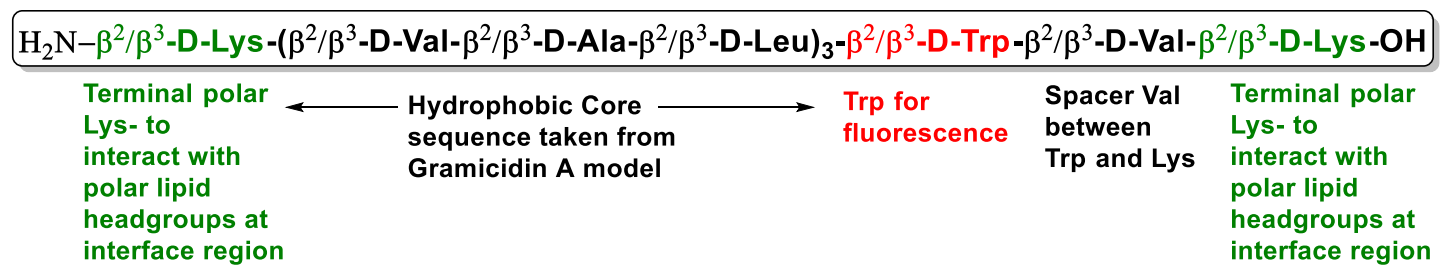

Figure 3.2: Schematic representation of general structure of a designed $\beta$-peptide based artificial transmembrane sequence

\subsection{Design of 10/12-helical 6-peptide based artificial transmembrane domains}

Since the very beginning, the investigation on the effect of helical macrodipole moment on different biochemical and physico-chemical properties of membrane protein has been the main point of interest for this project. Therefore, to shed light experimentally on this topic, it is necessary to be able to synthesize at least two different varieties of $\beta$-peptide based model transmembrane sequences, one of which should possess definitive helical macrodipole moment and the other should have no helical dipole moment. The idea is to perform similar experiments with these two types and if they exhibit considerably different results, it could be possible to relate the experimental differences to the helical macrodipole moment. In this process, the most challenging part is to design and synthesize $\beta$-peptide based model transmembrane sequence that has almost no helical macrodipole moment.

Reviewing the different helical secondary structures possible for $\beta$-peptides, it is evident that the alternate 10/12-helix has almost no helical macrodipole moment. The backbone of 10/12-helix is intrinsically the most stable secondary helical structure, but in presence of hydrophobic side chains, it does not hold anymore. So, there could be two main possibilities to design a $\beta$-peptide based transmembrane peptide that folds into an alternate 10/12 helix.

i) Peptides synthesized by alternating chirality of $\beta^{2}$-or $\beta^{3}$-amino acids: 
<smiles>[R]NC(=O)C[C@@H](C)NC(=O)CC([R])NC([R])=O</smiles>

Unlike $(R / S) \beta^{3} / \beta^{3}$-peptide<smiles>[R]NC(=O)[C@H](C)CNC(=O)[C@H]([R])CNC([R])=O</smiles>

Unlike (R/S) $\beta^{2} / \beta^{2}$-peptide

ii) Peptide synthesized by alternating $\beta^{2}$-and $\beta^{3}$-amino acids with uniform chirality:<smiles>[R]C(=O)NC([R])CC(=O)NCC([R])C(=O)NC</smiles>

Like $(S / S) \beta^{3} / \beta^{2}$-peptide<smiles>[R]C(=O)N[C@@H]([R])CC(=O)NCC([R])C(=O)NC</smiles>

Like $(R / R) \beta^{3} / \beta^{2}$-peptide

Figure 3.3: Schematic overview of the possible combinations to synthesize 10/12-helical $\beta$-peptides

Since, the $\alpha$-helical secondary structures are better preserved in homochiral peptides, ${ }^{[145]}$ so the idea of designing $\beta$-peptide based transmembrane peptides by alternating chirality is immediately dropped. Therefore, the only option left is the homochiral alternate $\beta^{2} / \beta^{3}$-peptides. Therefore, the planned structures of the $\beta$-peptide based transmembrane sequences are $\mathbf{P 2}$ '.

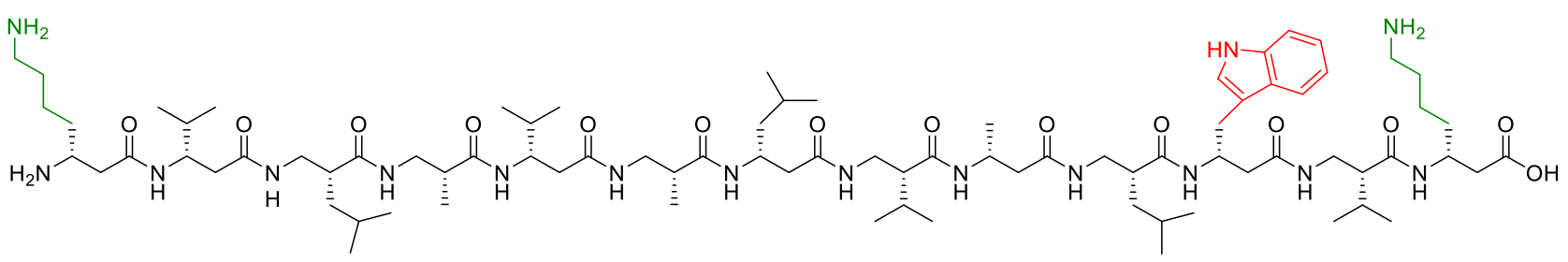

\section{P2'}

Figure 3.4: Preliminary design of the $\beta$-peptide based artificial 10/12-helical transmembrane domain, $\mathbf{P 2}$

However, the synthesis of this sequence was unachieved after several attempts with lot of different methods altering different parameters. So, some serious modifications had to be done to simplify the sequence as much as possible keeping the ultimate purpose in mind. The several modifications are:

i) Shortening the overall sequence length. 
ii) Omitting the polar amino acid residues (Lys-) at both the terminals, as the attachment of $\beta^{3}$ Lysines to the resin (2-chlorotrityl chloride), that was selected after several optimizations, was not successful. So, the end polar residues had to be eliminated from the sequence.

iii) Due to the elimination of polar amino acid residues, the hydrophobic amino acids had to be attached to the resin at the C-terminal. So, now there were two possibilities available, attaching a $\beta^{3}$-amino acid to make a $\beta^{3} / \beta^{2}$-peptide or attachment of a $\beta^{2}$-amino acid to synthesize a $\beta^{2} / \beta^{3}$ peptide. Both the peptides should technically exhibit the same 10/12-helical secondary structures, but in order to make sure that the $\beta^{3} / \beta^{2}$ and $\beta^{2} / \beta^{3}$-peptides have no different characteristics and effects on the desired physico-chemical and biochemical properties, both of the two types were designed to be synthesized and tested. Since, the rise/residue value for a 10/12-helical peptide is about $2.1 \AA$, so the length of the hydrophobic core of these peptides are approximately 18-19 A.

Therefore, the modified 10/12-helical alternate $\beta^{3} / \beta^{2}$ and $\beta^{2} / \beta^{3}$-peptides are $\mathbf{P} 2$ and $\mathbf{P} 3$.
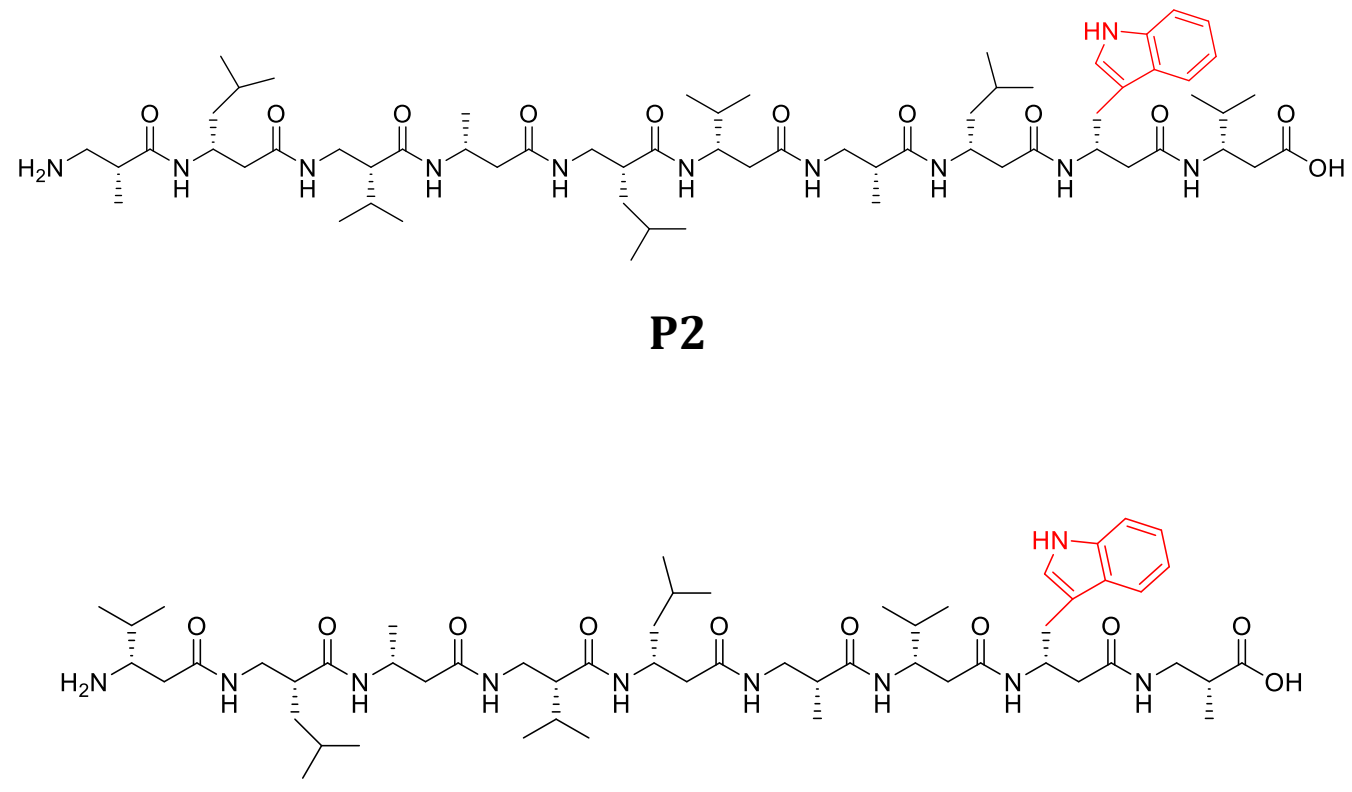

\section{P3}

Figure 3.5: Final sequences of the $\beta$-peptide based 10/12-helical artificial transmembrane domains; $\beta^{3} / \beta^{2}$-peptide (P2) and $\beta^{2} / \beta^{3}$-peptide (P3)

The main idea behind designing this peptide is to test any effect of helical macrodipole moment on transmembrane domain insertion into lipid membranes. It has already been discussed that when the hydrophobic core of the peptide is considerably shorter than the lipid bilayer thickness, a negative mismatch occurs. The negative mismatch can be compensated a number of 
ways, but one of the frequent outcomes of the negative mismatch is the non-insertion of the peptide into the lipid membrane and instead it takes a parallel orientation to the membrane at the membrane-water interface region. In order to compensate the negative mismatch, a probable solution is to reduce the bilayer thickness of the membrane. Moreover, as discussed earlier, short chain alcohols, like ethanol, has a distinctive ability to squeeze the bilayer thickness via keeping the fatty acid chains at an interdigitated state. So, the idea is to choose three different lipids in similar type with bilayer thickness gradually increasing in comparison to the length of the hydrophobic core of the transmembrane peptide. The lipid with thinnest bilayer should almost match with the hydrophobic core of the transmembrane peptide and the rest of the two lipids should have bilayer thickness gradually increasing up to a mismatch of approx. $10 \AA$. Therefore, minimum concentration of ethanol is used to compensate the negative mismatch between the peptide and the each lipid bilayers to the extent where the peptide is inserted into the membrane. Now, by comparing these results between a transmembrane peptide without macrodipole moment (10/12-helix) and a peptide with overall macrodipole moment (14-helix), if it is observed that to compensate similar negative hydrophobic mismatch (keeping in mind that the hydrophobic core length of both the peptides are the same and the three lipids used are also the same), there is difference in the required minimum concentration of ethanol, then it can easily be implied that due to the helical macrodipole moment either fascillitates or hinders the membrane insertion and spanning of the transmembrane domains (Figure 3.6)

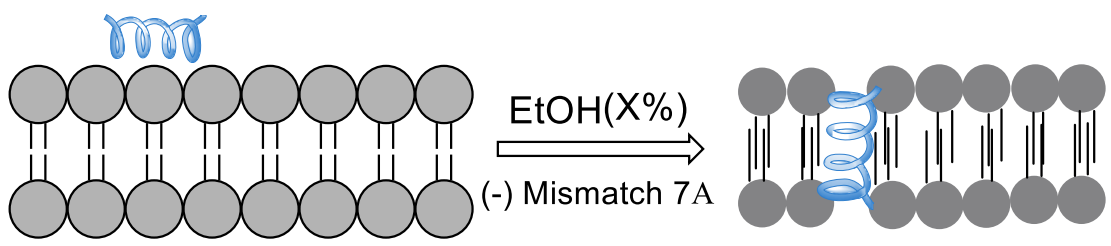

10/12-Helical Peptide with no macrodipole moment

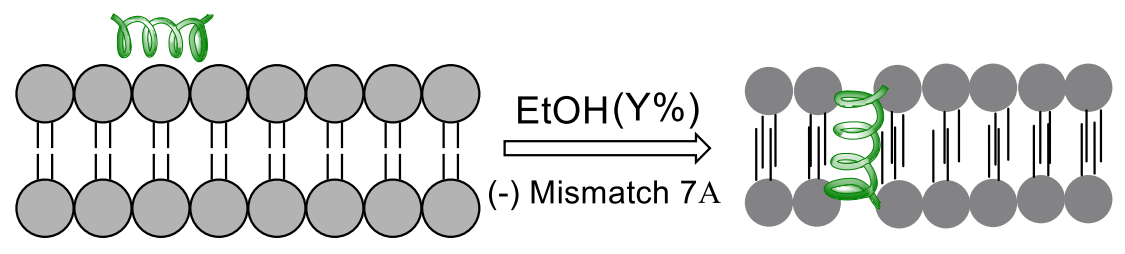

14-Helical Peptide with considerable macrodipole moment

Hydrophobic core length (10/12-helical peptide) = Hydrophobic core (10/12-helical peptide) Same lipid used for both peptides

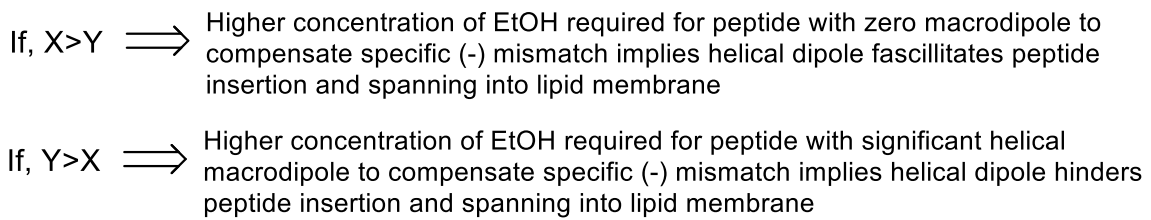


Figure 3.6: Schematic representation of the concept to determine effect of helical macrodipole moment on membrane insertion and spanning of transmembrane domains via compensation of negative hydrophobic mismatch with minimum concentration of ethanol

Besides the study on the effect of helical macrodipole moment of the designed $\beta$-peptide based transmembrane domains (P2 and P3) on lipid membrane insertion and spanning, it is also intended to investigate on the cellular uptakes of these types of neutral, hydrophobic short $\beta$ peptide sequences. Since, more than $90 \%$ of the established cell penetrating peptides carry several positive charges and are composed mostly with hydrophilic amino acid residues, like lysines or arginines, it woul dbe interesting to see if completely hydrophobic, chargeless, short $\beta$ peptides can penetrate cell membranes and enter the cytoplasm. Further to elucidate if there is any difference in uptake nature of the same cell lines between the $\beta$-peptide sequences with and without macrodipole moment. In that case, it would be possible to shed light on whether the macrodipole moment also regulate biochemical phenomena, like, cellular uptakes.

So, in this purpose, a short hydrophobic sequence of alternate $\beta^{2} / \beta^{3}$-peptide is designed that has no lysines or tryptophans in it (P6).

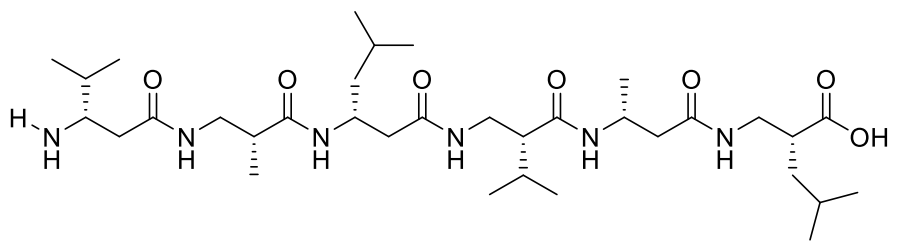

P6

Figure 3.7: 10/12 helical short $\beta$-peptide sequence as a candidate for cell penetrating peptide (C.P.P), P6

In order to quantify the cellular uptake by Fluorescence activated cell sorting (FACS) technique and visualize the peptide inside the cell by Laser confocal fluorescence microscopy, a fluorophore, 5(6)-carboxyfluorescein (5(6)-FAM) is attached to the N-terminal of the peptide P6 to give rise the fluorescently labeled derivative $\mathbf{P 7}$.

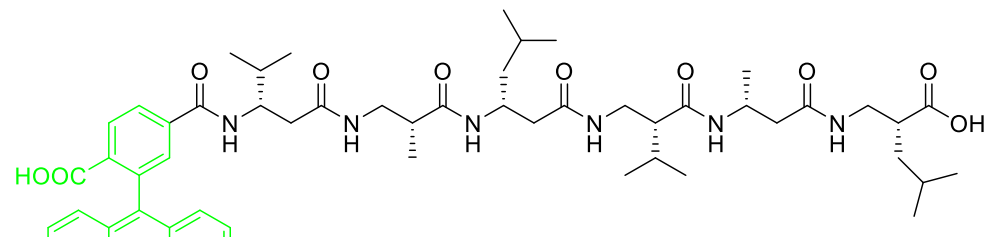


Figure 3.8: FAM-labeled 10/12 helical short $\beta$-peptide sequence as a candidate for cell penetrating peptide (C.P.P), P7

\subsection{Design of 14-helical 6-peptide based artificial transmembrane domains}

In correspondence to the designed $\beta$-peptide based artificial 10/12-helical transmembrane domain systems, the 14-helical transmembrane $\beta$-peptides are also designed following the same strategy. Since, to compare the results of the experiments with the 10/12-helical peptides, P2 and P3, a peptide P1, consists of a hydrophobic core composed of $\beta$ (Val/Ala/Leu) triad with a tryptophan towards the C-terminal. The hydrophobic length of the designed 14-helical peptide should also match with that of its 10/12-helical counterparts, so it also has to be approximately 18-19 A. The rise/residue for 14-helical peptide is about $1.6 \AA$, so the number of amino acid residues are increased to 12 amino acids in comparison to the 10/12-helical peptides, since the 10-12-helix is longer and narrower in nature with respct to the 14-helix. It has been observed that oligomer of homochiral $\beta^{3}$-amino acids form stable 14-helical secondary structures. Hence, the sequence of the $\beta$-peptide based 14-helical transmembrane peptide is shown in Figure 3.8.

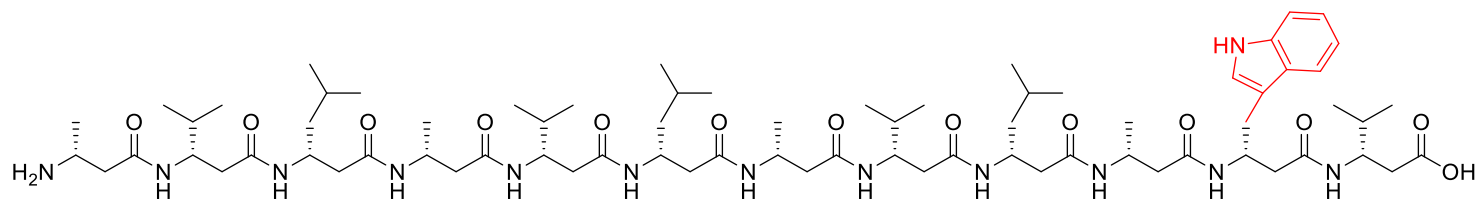

\section{P1}

Figure 3.9: $\beta$-peptide based 14-helical artificial transmembrane domain, P1

It is well established that the helical macrodipole moment is the summation of the individual dipole moments of the amide bonds of each amino acid residues. So, the longer the peptide, the more should be its overall helical macrodipole moment. Therefore, in order to make sure that dipole moment is the responsible factor behind any changes occurring during the physicochemical and biochemical investigations, two more $\beta$-peptides, P4 and P10, are designed. These hydrophobic cores of these two peptides are composed of only $\beta^{3}$-D-Val residues instead of the Val/Ala/Leu-triad. In addition, unlike P1, they have $\beta^{3}$-D-Lysines at the two termini followed by $\beta^{3}$-D-Tryptophans on each side. The only difference between the $\mathbf{P 4}$ and P10 is that hydrophobic 
core of P4 is longer with $19 \beta^{3}$-D-Val residues to reach a length of approx. 27-28 $\AA$, whereas, P10 has only $12 \beta^{3}$-D-Val residues with a length of approx. 18-19 A. So, in case helical macrodipole moment has any role in regulating the membrane insertion and spanning of the transmembrane domains, a difference in result should be noticed between two same peptide sequences with different chain length. Therefore, the two-peptide sequences P4 and P10 have been designed as shown in figure $\mathbf{3 . 1 0}$.

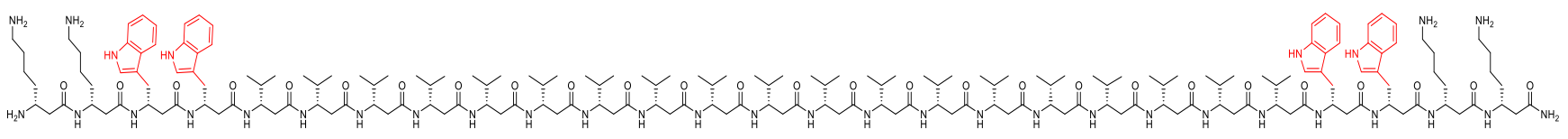

A

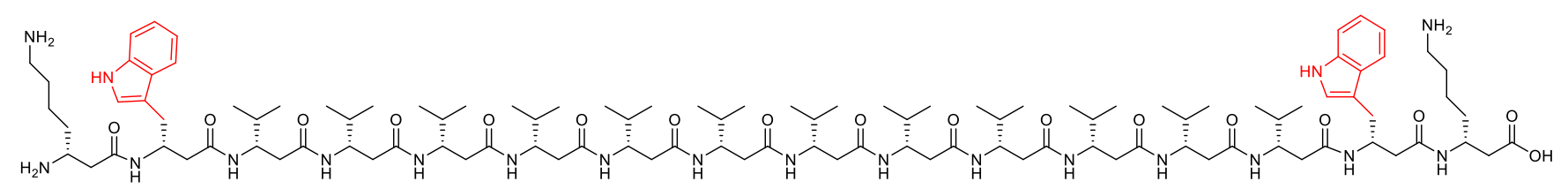

B

Figure 3.10: 14-helical $\beta^{3}(\mathrm{Val})$-peptide transmembrane domain sequences; the longer one, P4 (A) and the shorter one $\mathbf{P 1 0}(\boldsymbol{B})$

In addition of $\mathbf{P 4}$ and P10, two more short chain 14-helical $\beta^{3}$-peptides composed of Val/Ala/Leutriad, P8 and P9, were synthesized to compare the cellular uptake and cell penetration efficiency results with the short-chain alternate 10/12-helical $\beta^{2} / \beta^{3}$ (Val/Ala/Leu)-triad peptides, P6 and P7.

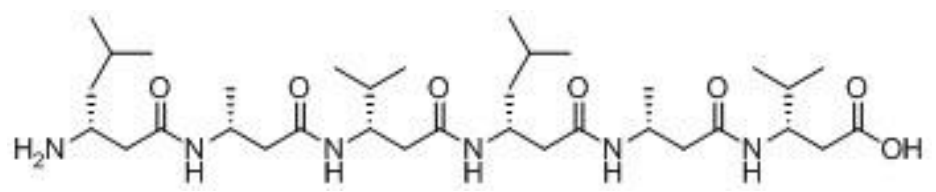




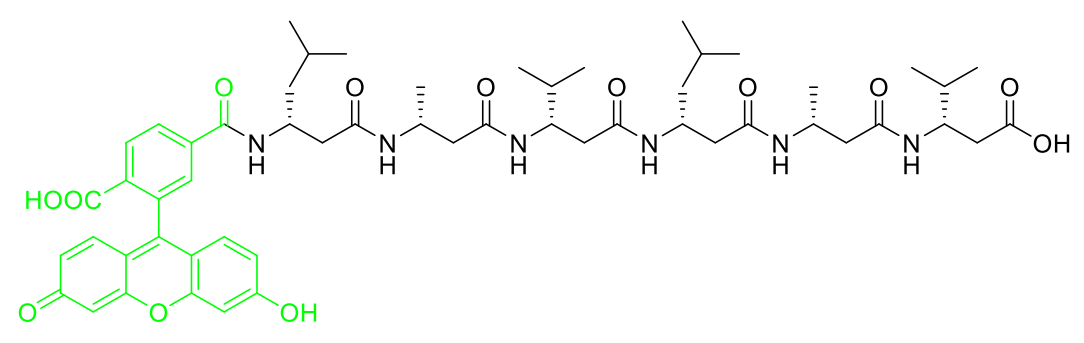

B

Figure 3.11: 14-helical $\beta^{3}$ (Val/Ala/Leu)-triad peptides for ell penetration studies; the unlabeled peptide $\boldsymbol{P 8}(\boldsymbol{A})$ and the FAM-fluorescently labeled peptide $\mathbf{P 9}(\boldsymbol{B})$

\subsection{Design of 12-helical 6-peptide based artificial transmembrane domains}

It is well known that the structural characteristic of 12-helix is profoundly different from that of a 14-helix. However, both the 14-helix and 12-helix have a common similarity amongst them and dissimilarity with the 10/12-helix: both of them have a considerable helical macrodipole moment arising due to the summation of the dipole moments of the individual amide bonds that the 10/12-helix lacks in. That is why similar to the 14-helix, study with the 12 -helix should establish the role of helical dipole moment in transmembrane peptide insertion and spanning in a stringer way. However, unlike the 14-helix, 12-helical peptide could not be designed with $\beta^{3}$ (Val/Ala/Leu) repetition and instead an oligomeric chain of $\beta^{3}$-Leu is found to form a stable 14helical peptide. Therefore, in analogy to 14-helical peptide $\mathbf{P 4}$ and P10, two 12-helical peptides, $\mathbf{P 5}$ and P11, have been designed where $\mathbf{P 5}$ is the longer one matching the hydrophobic core length of the peptide P4 and P11 with the peptide P10. The 12-helix is basically longer and narrower than that of the 14-helix with a rise/residue approx. $2.1 \AA$, so $15 \beta^{3}$-Leu residues have been used in $\mathbf{P 5}$ to approximately match the hydrophobic core length of $\mathbf{P 4}$, which is 29-30 and $9 \beta^{3}$-Leu residues have been added to P11 making it approx. 18-19 $\AA$ in length to mimic the hydrophobic core length of 14-helical peptide P10. Similar to the 14-helical $\beta^{3}$-Val peptide counterparts, P5 and P11 also have $\beta^{3}$-D-Lysines at the two termini and $\beta^{3}$-Tryptophans following the lysines at the two sides. 


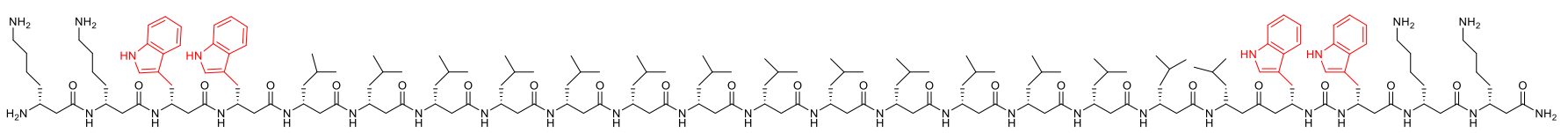

A

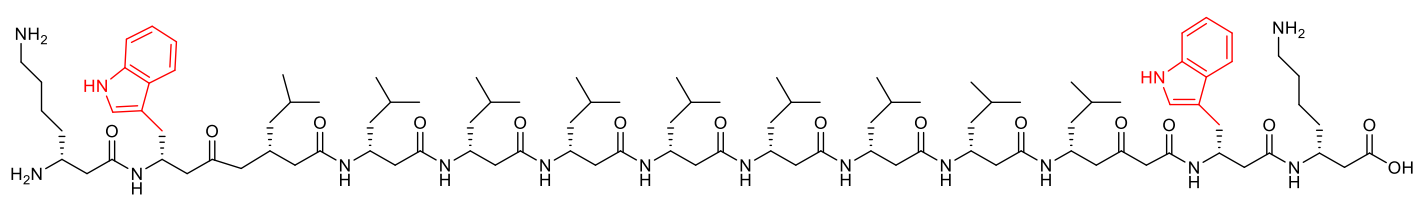

B

Figure 3.12: 12-helical $\beta^{3}$ (Val)-peptide transmembrane domain sequences; the longer one, P5 (A) and the shorter one P11 (B)

\section{4. $\beta$-Peptide: Synthesis}

\section{1 $\beta$-amino acids: a brief overview}

The synthesis of $\beta$-amino acids has been extensively studied in the last few decades and [8]. Due to the presence of an additional $\alpha$-methylene group, the $\beta$-amino acids represent a class of conformationally more flexible compounds as compared to their $\alpha$-amino analogs. Due to the extra $\alpha$-methylene group, unlike the $\alpha$-amino acids, the $\beta$-amino acids have two possible positions for functional groups; one at the $\alpha$-carbon with respect to the carbonyl group and the other at the $\beta$-carbon relative to the carbonyl group. If the substitution is at the $\beta$-carbon relative to the carbonyl group, it is known as the $\beta^{3}$-amino acids. On the other hand, if the substitution is at the $\alpha$-carbon with respect to the carbonyl group, it is known as the $\beta^{2}$-amino acids.<smiles>[R]C(N)C(=O)O</smiles>

$\alpha$-amino acids

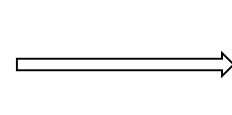<smiles>[R]OC(=O)CC([R])N</smiles>

$\beta^{3}$-amino acid

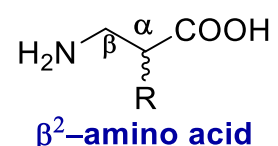

$\beta^{2}$-amino acid

Figure 4.1: Two major types of $\beta$-amino acids 


\subsection{Synthesis of $b^{3}$-amino acids}

There are several synthetic routes for the synthesis of $\beta^{3}$-amino acids. The $\beta$-amino acids can be produced enantioselectively from readily available $\alpha$-amino acids as the starting material. The $\alpha$ Amino acids are cheap, enantiomerically pure, commercially available compounds, which frequently serve as the starting materials for the synthesis $\beta$-amino acids [8]. Since the substitution is at the $\beta$-carbon with respect to the carbonyl group, so increment in one carbon chain length by Amdt-Eistert homologation reaction of corresponding N-protected $\alpha$-amino acids [9] followed by the Wolff rearrangement of the diazo ketone formed as the product of the first step, results in the formation of enantiopure $\beta^{3}$-amino acid analogs. [101] During the AmdtEistert homologation reaction, the starting D- $\alpha$-amino acids were reacted with the isobutyl chloroformate in presence of triethyl amine to obtain the activated ester. The diazomethane performed a nucleophilic attack on the activated esters to yield a diazoketone compound. In the next step of silver benzoate catalyzed Wolff rearrangement reaction, the diazoketone transformed into the desired enantiopure $\beta^{3}$-amino acids. There was no racemization observed in this two-step conversion and so the resulting $\beta^{3}$-amino acids have been enantiomerically pure. Several $\beta^{3}$-amino acids have been synthesized in bulk quantity with good yields (over 75\% on average) by this method with the help of orthogonal protection strategy to avoid unwanted side reactions.

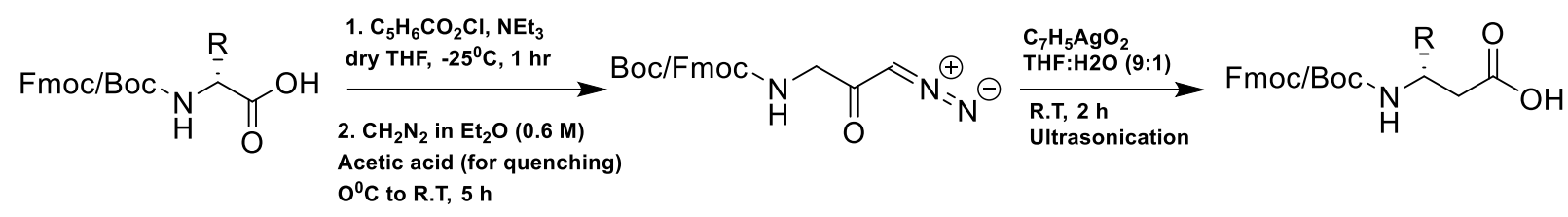

Figure 4.2: Two-step synthetic route for enantioselective synthesis of $\beta^{3}$-amino acids

\subsection{Synthesis of $\boldsymbol{6}^{2}$-amino acids}

\subsubsection{Route-1: Proline-catalyzed diastereoselective aminomethylation of aldehydes}

Unlike the $\beta^{3}$-amino acids, the $\beta^{2}$-amino acids could not be readily synthesized from the enantiopure $\alpha$-amino acid counterparts. Since, the substitution is at the $\alpha$-carbon with respect to the carbonyl group, so a simple extension of the carbon-chain length by homologation would 
only result in $\beta^{3}$-amino acids and not the desired $\beta^{2}$-amino acid analogs. Therefore, a total retrosynthetic approach was necessary that also has to be enantioselective in nature. One of the more popular routes to access protected $\beta^{2}$-amino acids was reported by Gellman et. al. $[146,147]$ via proline-catalyzed diastereoselective aminomethylation of aldehydes.

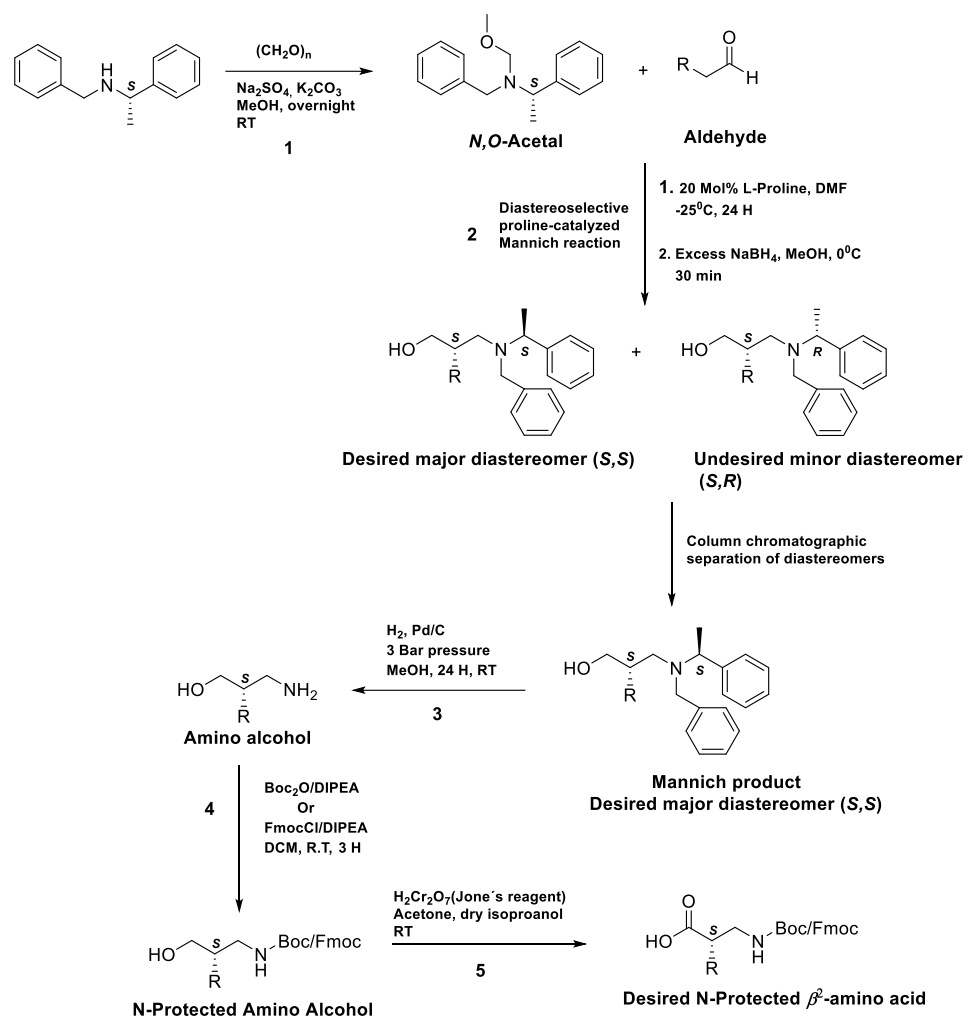

Figure 4.3: Synthetic route to access $N$-protected $\beta^{2}$-amino acids by proline-catalyzed diastereoselective aminomethylation of aldehydes via Mannich reaction

The method uses commercially available chiral N-benzyl- $\alpha$-Methylbelzylamine to react with para-formaldehyde in anhydrous $\mathrm{MeOH}$ to yield the chiral $\mathrm{N}, \mathrm{O}$-acetal compound (reaction-1 in Fig. 4.3). This chiral $\mathrm{N}, \mathrm{O}$-acetal is the precursor for the imminium cation generated in situ. Mannich reaction took place in the next step (reaction-2 in Fig. 4.3), but the aldehydes required for this reaction were sometimes commercially not available, so the commercially unavailable aldehydes were synthesized from their corresponding alcohols with PCC as the main reagent (not shown in scheme in Fig. 4.3). Mannich reaction occured between the chiral imminium electrophile and the enamine generated due to the proline-catalyzed aminomethylation of the aldehyde. Although, it was mentioned that the process is diastereoselective [146, 147], but the extent of selectivity has not been considerably good and the reaction produced significant amount of the $R, S$-diastereomer as the undesired minor product along with the desired $S, S$ diastereomer as the major product. The minor product was also visible under TLC. Therefore, 
these diastereomers needed to be separated via column chromatography. This had been a very tedious task and separation of the two almost inseparably close spots in TLC for the two diastereomers had not completely been successful. This yielded a mixture of diastereomers as the products of Mannich reaction. In the next step (reaction-3 in Fig. 4.3), the benzyl groups were reduced via hydrogenation with $\mathrm{Pd} / \mathrm{C}$ to get a $\gamma$-amino alcohol. The N-Boc or N-Fmoc protection was carried out in the next step (reaction-4 in Fig. 4.3) followed by the oxidation with Jones reagent to access the desired $N(B o c) /(F m o c)-\beta^{2}$ amino acids (reaction-5 in Fig. 4.3). The overall yield after the five steps and diastereomeric separation has not been quite impressive (below 30\% on average). The orthgonal protection strategy was used to prevent unwanted side reactions.

Although the desired $\beta^{2}$ amino acids could be synthesized by this method, but the method had several major disadvantages:

i) Involved 5 synthetic steps with poor overall yield.

ii) Diastereoselective Mannich reaction produced considerable mixture of diastereomers that could not completely be separated by column chromatography purification and had to proceed further with a product as a mixture of two diastereomers. This has a strong chance that the ultimate product, $\beta^{2}$ amino acids, might also be a mixture of two enantiomers. In that case, working with such an enantiomeric mixture would confuse the results of the further experiments and nothing can be concluded.

iii) involves heavy metal based toxic reagents like, PCC and Jones reagent, which gives rise to considerable amount of heavy metal based tar-like toxic wastes.

Therefore, development of an alternative route to access the desired $\beta^{2}$ amino acid building blocks was required.

\subsection{Route-2: Asymmetric $\alpha$-alkylation of 6 -alanine using Pseudoephedrine as a chiral auxiliary}

Due to the major disadvantages of the method that made use of proline-catalyzed diastereoselective aminomethylation of aldehydes, a different route was devised to access enantiomerically pure $\beta^{2}$-amino acids. This method relied upon asymmetric $\alpha$-alkylation of $\beta$ alanine using Pseudoephedrine as a chiral auxiliary. ${ }^{[148]}$ D-Pseudoephedrine $([1 S, 2 S]-(+))$ is a commodity chemical employed in over-the-counter medications with annual worldwide 
production in excess of 300 metric tons. L-Pseudoephedrine is also readily available in bulk and is inexpensive. Pseudoephedrine is widely employed as a chiral auxiliary in diastereoselective alkylation reactions and provides ready access to enantiomerically enriched carboxylic acids, aldehydes, ketones, and alcohols. [149-152]

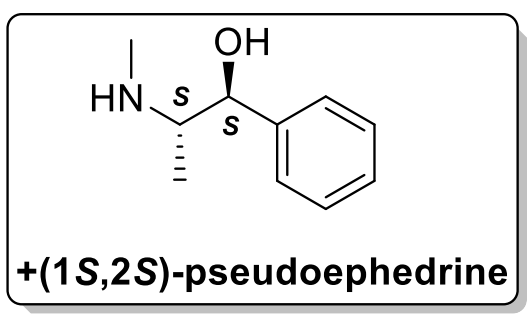

Figure 4.4: Myer's chiral auxiliary Pseudoephedrine

So, the idea was to start with inexpensive and readily available compound, N(Boc)- $\beta$-alanine and convert it acid chloride by treating with pivaloyl chloride and triethyl amine followed by the treatment with the desired specific enantiomer $[(1 S, 2 S)(+)]$ of pseudoephedrine to generate a specific enantiomer of N(Boc)-deprotected pseudoephedrine amide (reaction-1 in Fig. 4.5). In the next step, the important asymmetric $\alpha$-alkylation of the pseudoephedrine amide is carried out by treating the latter with the desired alkyl halide (selected depending upon the amino acid side chain) in presence of n-butyl lithium (2.5 M in n-hexane) and lithium chloride at $-78^{\circ} \mathrm{C}$. The same reaction was also tried with LHMDS at $-5^{0}$ to $0^{\circ} \mathrm{C}$ temperature (reaction-2 in Fig 4.5). In addition, at the last step, detachment of the chiral auxiliary, $+1 \mathrm{~S}, 2 \mathrm{~S}$-Pseudoephedrine, was carried out by refluxing in water for 3 days (reaction 3 in Fig 4.5).
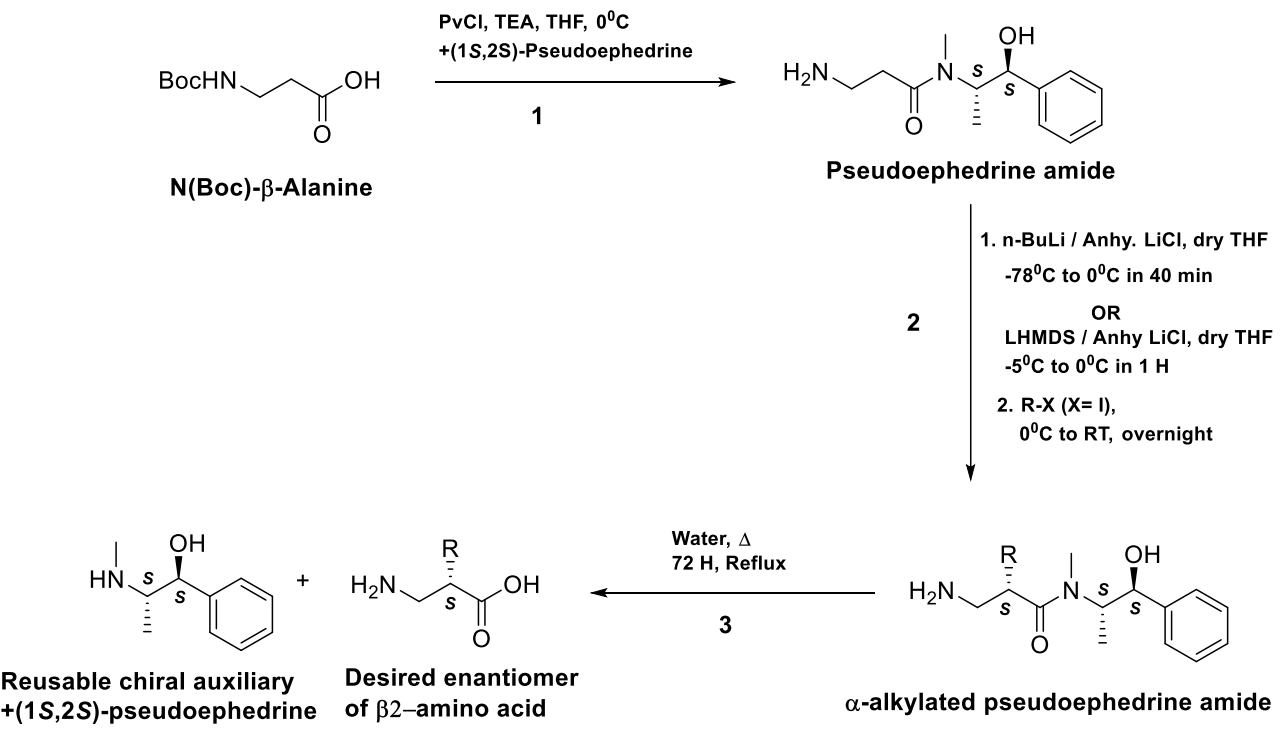
Figure. 4.5: Synthetic route to access $\beta^{2}$-amino acids by asymmetric $\alpha$-alkylation of $\beta$-alanine using Pseudoephedrine as a chiral auxiliary

The route initially came up as a very efficient one. This had several advantages over the previously described route-1:

i) It only consisted of three-steps.

ii) The overall yield was over $65 \%$ on average.

iii) Only cheap and readily available starting material and reagents were required with no use of any kind of toxic chemicals.

iv) It required less time and effort to synthesize the target.

Nevertheless, there was one big disadvantage attached to this route. The enantioselectivity of the chiral auxiliary, Pseudoephedrine, for this method was not high enough. The enantiomeric excess was determined via chiral HPLC using Chiracel-OD column and was found to be around 77:23. Therefore, considerable quantity of the undesired enantiomer was also generated as a minor product and at the end a mixture of two enantiomers were obtained. The separation of these two enantiomers was also not trivial. However, in comparison to the route-1, this method was far more successful considering all the other advantages.

\subsection{Synthesis of 14-helical 6-peptides}

\subsubsection{Synthesis of 14-helical $\boldsymbol{B}^{3}$-(Val)-peptide:}

The 14-helical $\beta^{3}$-(Val)-peptides, P4 and P10, have been synthesized by microwave-assisted manual solid phase peptide synthesis (SPPS) ${ }^{[155-158]}$ Usually the synthesis of long hydrophobic transmembrane peptide sequence is quite challenging due to their inherent inclination towards random self-aggregation because of high hydrophobicity. However, the following SPPS method (Fig. 4.6) using MBHA rink-amide resin (loading capacity $0.57 \mathrm{mmol} / \mathrm{g}$ ) has been quite successful. 


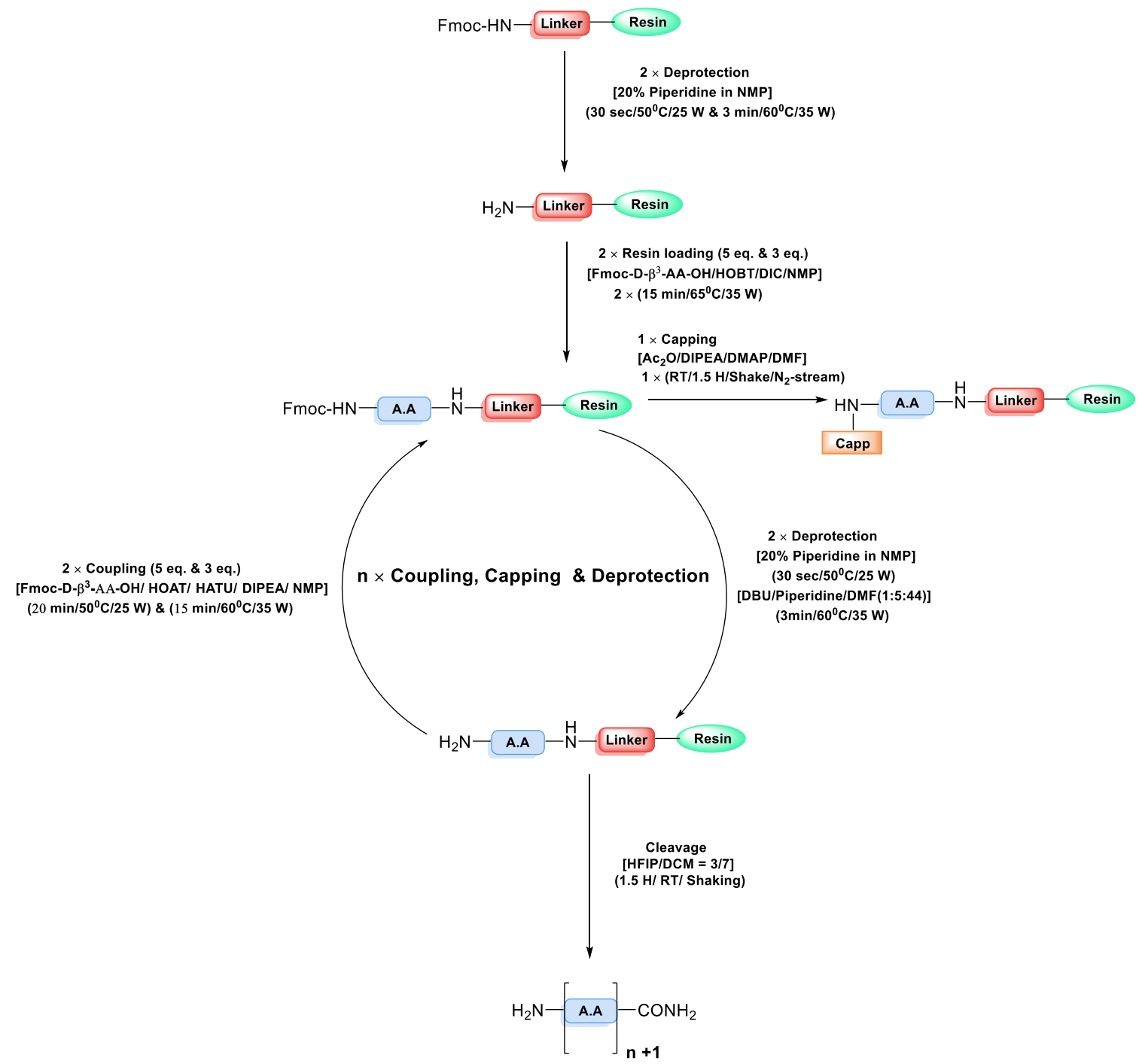

Fig. 4.6: Schematic representation of 14-helical $\beta^{3}$-(Val)-peptides by microwave-assisted manual SPPS

The SPPS cycle began with the deprotection of the Fmoc-group of the MBHA rink amide resin followed by loading the resin with the first D- $\beta^{3}$-Fmoc-Lys (Boc)-OH amino acid. The free amines were capped using a mixture of acetic anhydride, DMAP and DIPEA in NMP followed by the deprotection of the Fmoc-group under basic conditions with a cocktail of $20 \%$ piperidine in NMP and 2\% DBU in NMP. The subsequent coupling of the next amino acids were carried out twice with a coupling cocktail of HATU/HOAT/DIPEA at $60^{\circ} \mathrm{C}$ for $20 \mathrm{~min}$ each. The cycles of couplingcapping-deprotection was repeated until the target sequence was achieved. At the end, the target $\beta$-peptide was cleaved from the resin under acidic condition with a cocktail of HFIP/DCM (3:7) to obtain the free target 14-helical $\beta^{3}(\mathrm{Val})$-peptide. The overall yield for the shorter sequence, P10, was comparatvely higher than that of the longer peptide, P4. 


\subsubsection{Synthesis of 14-helical B $^{3}$-(Val/Ala/Leu) triad-peptide:}

Unlike the 14-helical $\beta^{3}$ (Val)-peptides, the $\beta^{3}$-(Val/Ala/Leu) triad peptides, P1, P8, P9, were synthesized by a non-microwave-assisted manual SPPS protocol. These sequences were also tried to synthesize with the same method as the 14-helical $\beta^{3}$ (Val)-peptides, but the yields were extremely poor. So, a different method was adopted and optimized. [8] Instead of MBHA rink amide resin, 2-chlorotrityl chloride resin (loading capacity $1.63 \mathrm{mmol} / \mathrm{g}$ ) was used (Fig 4.7).

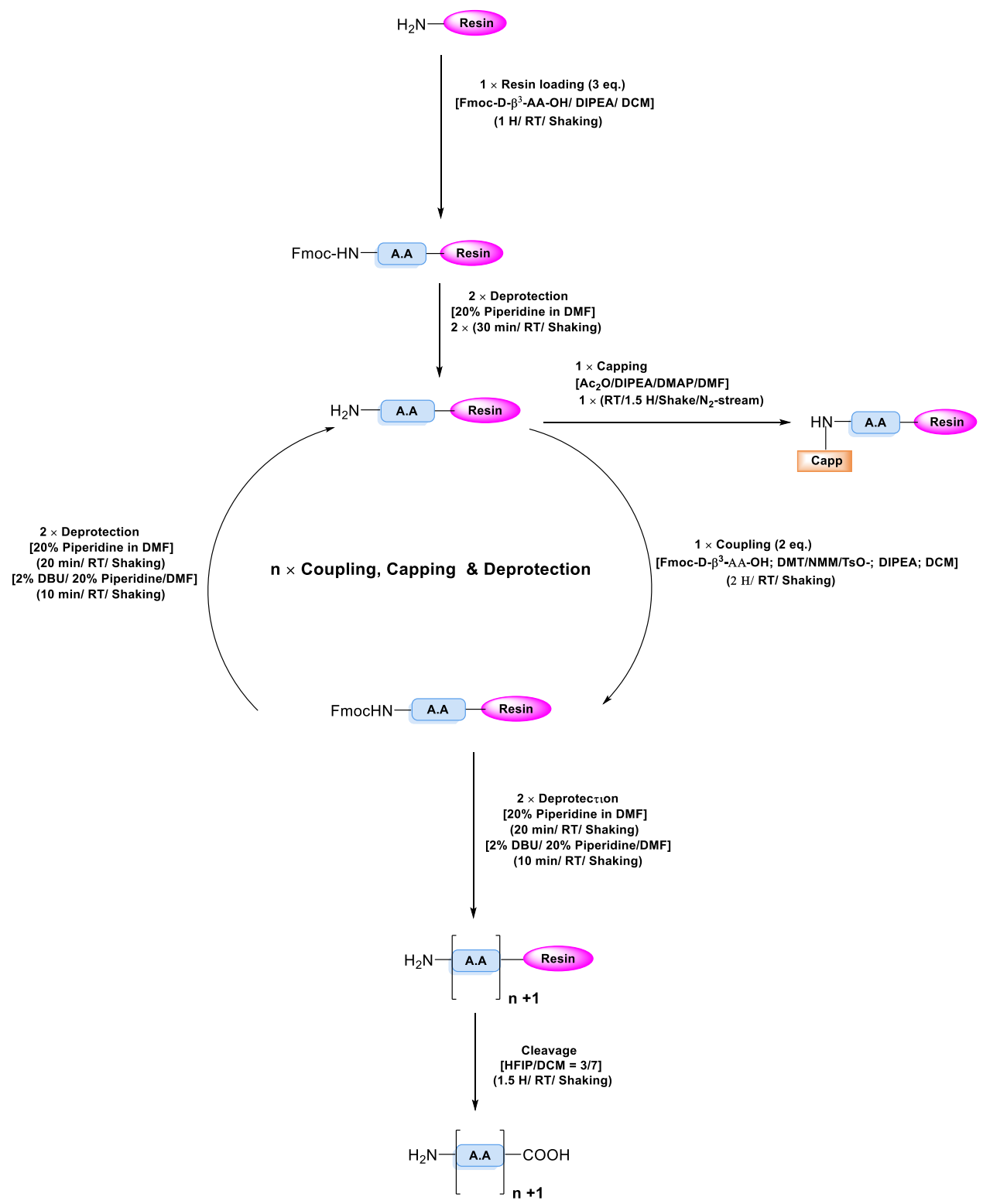

Figure 4.7: Schematic representation of the 14-helical $\beta^{3}$-(Val/Ala/Leu)-triad-peptides by nonmicrowave-assisted manual SPPS 
The synthesis of these target triad sequences begin with loading the resin with the first amino acid $\beta^{3}$-D-Fmoc-Val-OH with DIPEA shaking at room temperature. Then the unreacted free amines were capped at room temperature for $1.5 \mathrm{~h}$ using a capping mixture of acetic anhydride/DMAP/DIPEA in DMF followed by the deprotection of the Fmoc-group at room temperature in two steps under basic condition, first with $20 \%$ piperidine in DMF for 20 min and then with a cocktail of $2 \%$ DBU in DMF and 20\% piperidine in DMF for 10 min. The next coupling was subsequently carried out with the other Fmoc-protected amino acids using a synthesized coupling reagent, DMT/NMM/TsO- for $2 \mathrm{~h}$ at room temperature. This cycle of coupling-cappingdeprotection was continued until the target sequence was achieved. At the end, the peptide was cleaved from the resin with a cleavage cocktail of HFIP/DCM (3:7). The overall yield of the peptides were comparatively higher than that of the $\beta^{3}$-Val peptides by this non-microwaveassisted manual SPPS.

\subsection{Synthesis of 12-helical 6-peptides}

Similar to the 14-helical $\beta^{3}$-(Val)-peptides, the 12-helical $\beta^{3}$-(Leu)-peptides, P5 and P11, were also synthesized by microwave-assisted manual SPPS. The coupling of Fmoc-D- $\beta^{3}$-(Leu)-OH were even more difficult as the peptides with more hydrophobic side-chain were more prone towards random self-aggregation. Therefore, a different resin, Nova PEG rink amide LL, was used and higher coupling temperature as well as higher amino acid equivalence were used to successfully access the designed 12-helical peptides (Fig. 4.8). 


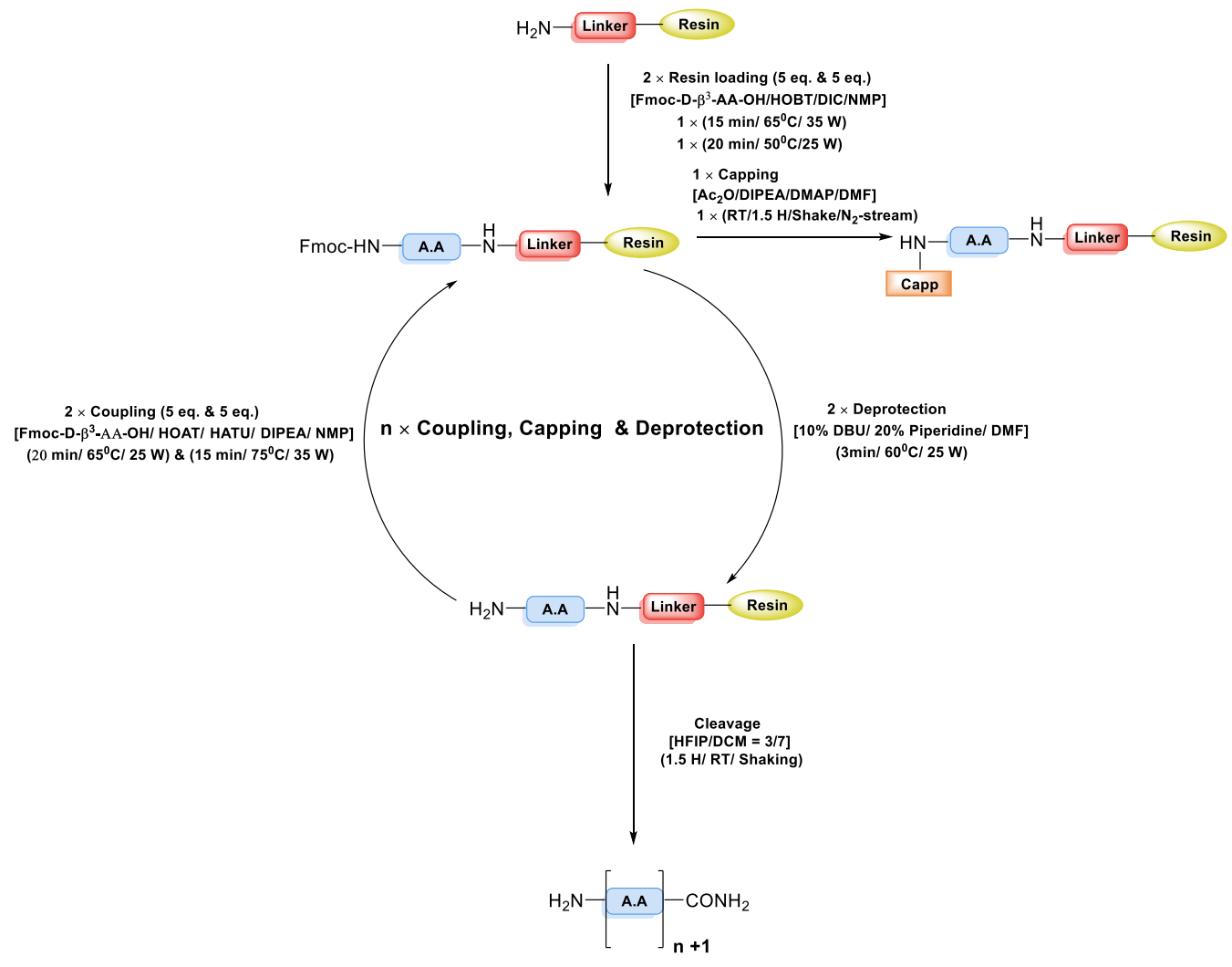

Figure 4.8: Schematic representation of 12-helical $\beta^{3}$-(Leu)-peptides by microwave-assisted manual SPPS

Unlike, the MBHA rink amide resin used to synthesize 14-helical $\beta^{3}$-(Val)-peptides, the Nova PEG rink amide LL resin does not require initial Fmoc-deprotection and hence the SPPS cycle begins with loading the resin with D- $\beta^{3}$-Fmoc-Lys (Boc)-OH amino acid. The free amines were capped using a mixture of acetic anhydride, DMAP and DIPEA in NMP under $\mathrm{N}_{2}$-gas bubbling at room temperature for $1.5 \mathrm{H}$ followed by the deprotection of the Fmoc-group under basic conditions with a cocktail of $20 \%$ piperidine and $10 \%$ DBU in NMP. The coupling of the next amino acids were performed twice each with 5 eq. of the Fmoc-amino acids. So, a higher equivalence of amino acids were used for 12-helical peptide synthesis as the efficiency of coupling is much poor than that of the 14-helical $\beta^{3}$-(Val)-peptides. Unlike the 14-helical $\beta^{3}$-(Val)-peptides, it also required much higher temperature, up to $75^{\circ} \mathrm{C}$, for a successful coupling. The cappingdeprotection-coupling cycle was continues until the target sequence length was achieved. The target sequence was cleaved from the resin using an acidic cleavage cocktail composed of HFIP/DCM (3/7). The overall yield was still much lower than that of the 14-helical $\beta^{3}$-(Val)peptides. 


\subsection{Synthesis of alternate 10/12-helical 6-peptides}

\subsubsection{Heavy-fluorous-tagged solution phase synthesis}

Unlike the 14- or 12-helical peptides, there have been very less reports on the effective synthesis of alternate 10/12-helical $\beta$-peptides of comparable sequence length. Many trials with different coupling conditions and different resins in microwave-assisted SPPS were completely unsuccessful. So, an unconventional method, based on heavy-fluorous-tagged solution phase strategy, for synthesizing the unique 10/12 helical peptides was adopted (Figure 4.9). [159]

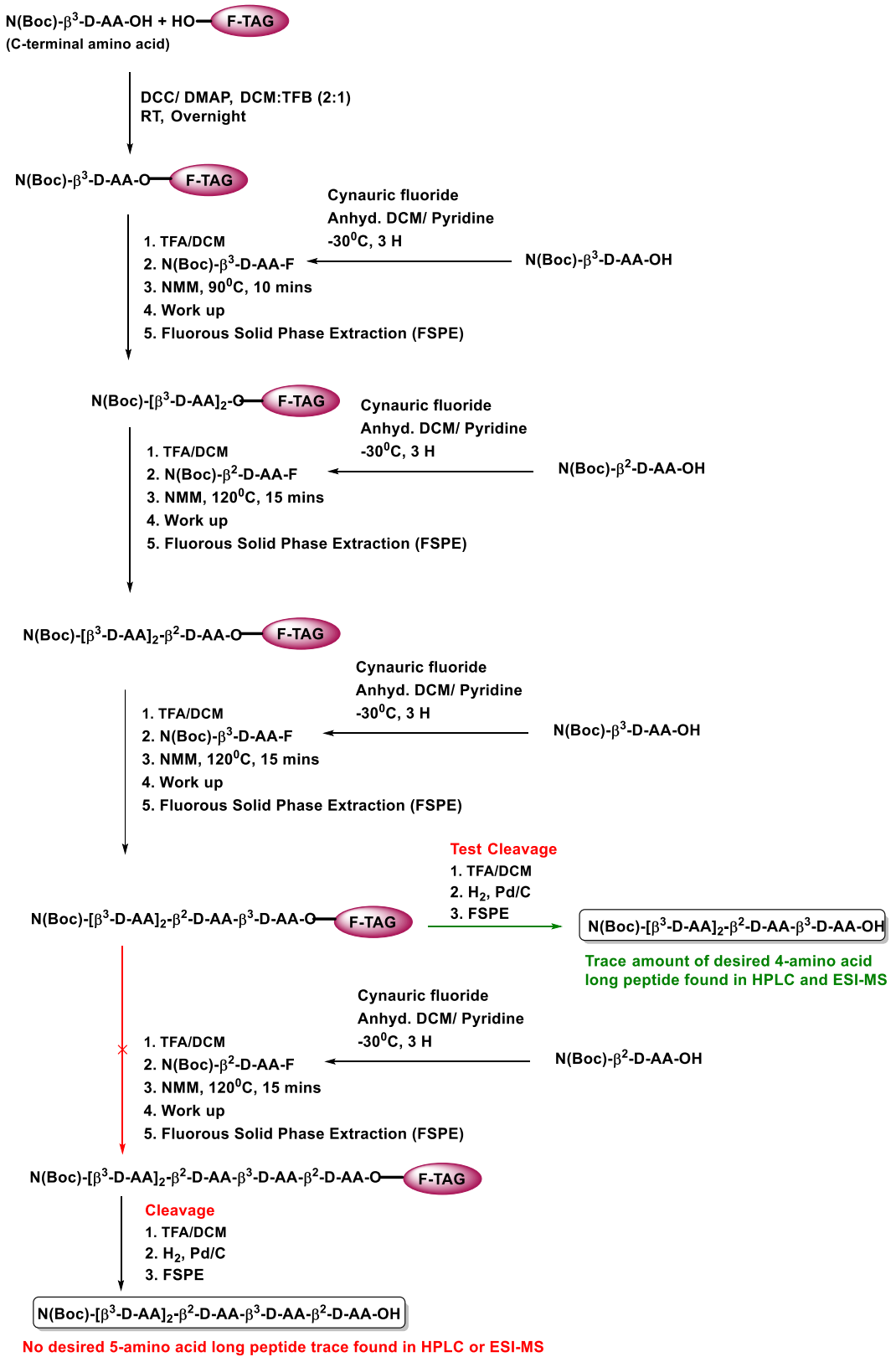


Figure 4.9: Schematic representation of the heavy-fluorous-tag-assisted solution phase peptide synthesis for alternate 10/12-helcal peptides [159]

This method was fundamentally developed for the glass micro-reactor system and was comparatively more efficient in that system. However, alternate $10 / 12$-helical $\beta$-peptides were tried no longer than 4-amino acid long sequences by this method. Since the typical glass-microreactor, system was not available for this project, so the method was tried with some minimal optimization of the conditions in solution phase using regular glass apparatus. It was a tedious method, as after each coupling step, the desired peptide fractions had to be purified by fluorous solid phase extraction (FSPE) technique. This also reduced the yield to considerable extent. But as there was no other reported method available, so it was the only option at that moment.

The method started with the attachment of the first $\mathrm{N}(\mathrm{Boc})-\beta^{3}$-D-amino acid to the heavy fluorous tag (a benzyl alcohol derivative). At the next step, the N(Boc)-was deprotected under acidic condition using TFA/DCM. All the $\beta^{3}$ and $\beta^{2}$-amino acids were converted into their acid fluoride counterparts by using cyanuric fluoride at $-30^{\circ} \mathrm{C}$ for 3 hours. The acid fluorides were more reactive than that of the acids, and since the coupling between $\beta^{3-} \beta^{2}$-amino acids were energetically not favourable, so the coupling between the two amino acids were not effective and one of them had to converted to the acid fluoride. Therefore, the coupling between the fluoroustagged $\beta^{3}$-amino acid and the incoming $\beta^{3}$-amino acid fluoride was carried out at $90^{\circ} \mathrm{C}$ for 10 minutes followed by standard work up and purification of the fluorous tagged-desired di-peptide fragment by FSPE. Next, the N(Boc)-deprotection was carried out using the same acidic condition followed by the coupling of an incoming $\beta^{2}$-amino acid fluoride. The coupling between the fluorous-tagged $\beta^{3}$-dipeptide and incoming $\beta^{2}$-amino acid fluoride was performed at $120^{\circ} \mathrm{C}$ for 15 minutes followed by work-up and purification of the desired tri-peptide fragment by FSPE. So, the cycle of coupling between $\beta^{3}$ and $\beta^{2}$-amino acids were continued until a tetrapetide was synthesized. At this stage, formation of the tetra-peptide was confirmed by RP-HPLC and ESI-MS after a small portion was cleaved from the fluorous-tag via hydrogenation with hydrogen and $\mathrm{Pd} / \mathrm{C}$ overnight under 3 Bar pressure. But unfortunately, no trace of a penta-peptide product was obtained from RP-HPLC and ESI-MS. Therefore, only an alternate 10/12-helical tetra-peptide composed of alternating $\beta^{3} / \beta^{2}$-amino acids could successfully be accessed via this heavyfluorous-tag-assisted solution phase synthesis strategy. 


\subsubsection{Non-microwave-assisted manual SPPS}

After getting unsuccessful in synthesizing the target 10/12-helical transmembrane $\beta$-peptides with microwave-assisted SPPS as well as heavy-fluorous-tag-assisted solution phase peptide synthesis strategies, a new route was tried (Figure 4.10), as reported by Kolesinska et. al. [8]

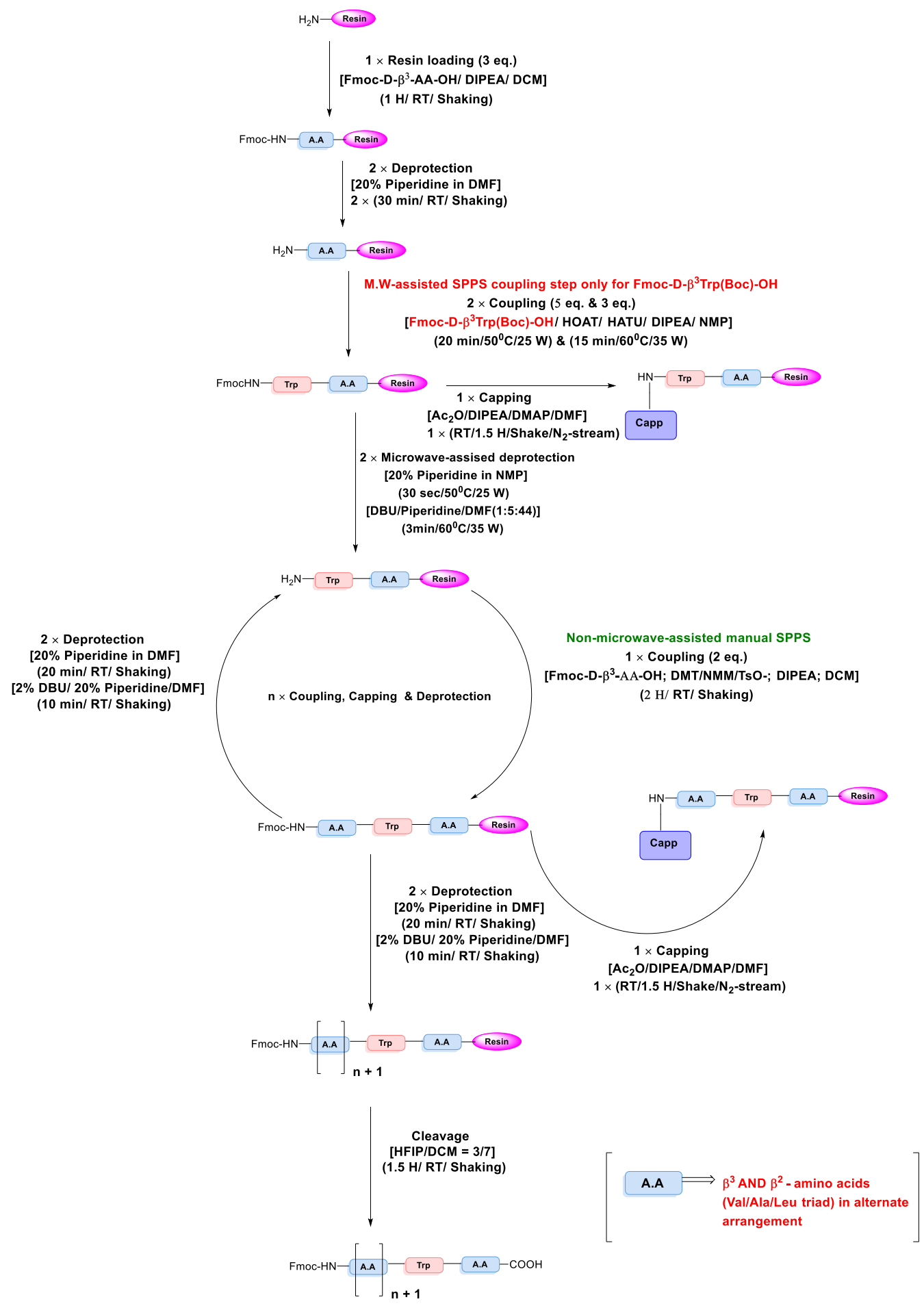


Figure 4.10: Schematic representation of non-microwave-assisted manual SPPS synthetic strategy to synthesize alternate 10/12-helical transmembrane $\beta$-peptides composed of Val/Ala/Leu triads. $[8]$

The synthesis by non-microwave-assisted SPPS was initially tried by block co-polymer synthesis strategy via coupling a resin-cleaved alternate 10/12-helical penta-peptide with another solidsupported 10/12-helical hexa-peptide under similar conditions as well as under an increased temperature to $50^{\circ} \mathrm{C}$ for 30 minutes. Since, the coupling was getting more and more inefficient with the increase of chain length starting from hexa-peptide, so the synthesis by blockcopolymer strategy was taken into account. But none of the trials resulted in a successful synthesis of the desired alternate 10/12-helical transmembrane sequence. The higher temperature reduces the probability of random peptide aggregation during SPPS, so an elevated temperature was used. However, that did not help.

Hence, the synthesis was continued in linear chain coupling fashion, the temperature was kept at room temperature, and the coupling time was increased to $2 \mathrm{H}$ along with a capping time for 1$1.5 \mathrm{H}$ at room temperature. Therefore, the method begins with loading the first amino acid, $\beta^{3}$-DVal/Ala/Leu or $\beta^{2}$-D-Val/Ala/Leu, to 2-chlorotrityl chloride resin (loading capacity $=1.63$ $\mathrm{mmol} / \mathrm{g}$ ) in presence of DIPEA at room temperature. The loaded resin was deprotected twice for 30 minutes with 20\% piperidine in DMF. Now the loaded resin was ready to undergo further coupling cycles. The synthesis of 10/12-helical hexa-peptide composed of pure hydrophobic sequences of alternate $\beta^{2} / \beta^{3}$ (Val/Ala/Leu/ triad (P6 and P7) was carried out successfully with very promising yields. The loaded resin was coupled with the target $\beta$-amino acid residue ( $\beta^{2}$ or $\beta^{3}$-Val/Ala/Leu in alternate fashion) in presence of a newly developed coupling reagent, DMT/NMM/TsO-, that had to synthesized at laboratory due to commercial unavailability ${ }^{[\mathbf{1 6 0}]}$ and DIPEA at room temperature for $2 \mathrm{H}$. The coupling was followed by capping of the unreacted free amines by the capping cocktail, acetic anhydride/DIPEA/DMAP/DMF, at room temperature under pure $\mathrm{N}_{2}$-bubbling for 1-1.5 $\mathrm{H}$. Next, the double deprotection was carried out; first with $20 \%$ piperidine in DMF for $20 \mathrm{~min}$ at room temperature and second with a cocktail of $20 \%$ piperidine in DMF and 2\& DBU in DMF for $10 \mathrm{~min}$. at room temperature. This cycle of couplingcapping-deprotection was continued until the target sequence was obtained. The peptide was cleaved form the resin under acidic conditions using HFIP/DCM (3/7) to yield the desired peptide as an acid at the C-terminal. Therefore, the target alternate 10/12-helical peptides composed of only hydrophobic alternate $\beta^{2} / \beta^{3}$-Val/Ala/Leu triads was not a problem. Nevertheless, the target peptides, P2 and P3, were 9-10 amino acid long. Synthesis of these 
longer sequences proved to be extremely challenging. The initial challenge was to elongate the chain length from 6 to 10 amino acid. After that was accomplished, the next challenge was to insert a $\beta^{3}$-D-Trp residue towards the $\mathrm{C}$ - or $\mathrm{N}$-termini. The presence of Trp was necessary, as the plan was to use Trp-fluorescence to check the position of the peptide whether in aqueous region or inside lipid vesicle (hydrophobic region). However, after several trials with various coupling conditions, the coupling of $\beta^{3}$-D-Trp to the 2-chlorotrityl resin or to other solid supported $\beta^{3}$-DVal/Ala/Leu or $\beta^{2}$-D-Val/Ala/Leu amino acid residues was not successful in non-microwaveassisted manual SPPS. Therefore, after loading the first $\beta^{2}$ or $\beta^{3}$-Val/Ala/Leu hydrophobic amino acid residue to the resin by non-microwave-assisted manual SPPS, the strategy was changed to microwave-assisted manual SPPS and the desired Trp-residue was coupled to the solid supported $\beta^{2}$ or $\beta^{3}$-amino acids by the method adapted to synthesize $\beta^{3}$-D-Val-peptides (P4 and P10). The coupling reagent was changed to DIPEA/HATU/HOAT and the coupling was performed two times with 5 eq. and 3 eq. of amino acids under $\left(20 \mathrm{~min} / 50^{\circ} \mathrm{C} / 25 \mathrm{~W}\right)$ and $\left(15 \mathrm{~min} / 60^{\circ} \mathrm{C} / 35 \mathrm{~W}\right)$ microwave conditions, respectively. The coupling was carried out manually without microwave for $1.5 \mathrm{H}$ with the same capping cocktail. The deprotection was again carried out twice with $20 \%$ piperidine in NMP and $20 \%$ piperidine in DMF + DBU/piperidin/DMF (1/5/44), under microwave conditions of $\left(30 \mathrm{sec} / 50^{\circ} \mathrm{C} / 25 \mathrm{~W}\right.$ ) and (3 $\min / 60^{\circ} \mathrm{C} / 35 \mathrm{~W}$ ), respectively. After the successful attachment of the Trp-towards the Cterminal of the growing alternate 10/12-helical peptide chain, the coupling strategy was again shifted to the non-microwave-assisted manual SPPS, just as the P6 and P7, and the couplingcapping-deprotection cycles were continued until the desired peptide sequences (P2 and P3) were obtained (Figure 4.10). The yield for the synthesis of P2 and P3 were comparatively much lower than that of $\mathbf{P 6}$ and P7, but the desired peptide could be purified by RP-HPLC and was confirmed by ESI-MS and HR-MS spectroscopy.

\subsection{Attachment of fluorophores to short-chain B-peptides}

In order to quantitively estimate the cellular uptake and cell penetration of the short-chain 14helical and alternate 10/12-helical $\beta$-peptides, P8 and P6, respectively, by a special type of flow cytometry technique, fluorescence-activated cell sorting (FACS), the peptides were labeled with 5(6)-carboxyfluorescein at the N-terminal and were converted to fluorescently labeled derivative peptides, $\mathbf{P 9}$ and $\mathbf{P 7}$. The fluorescent labeling of the peptides would allow them to be visualized under laser scanning confocal fluorescence microscopy imaging. This imaging would enable us to actually see the cellular location of the peptides and would determine whether the 
peptides were actually penetrated inside the cell to reach cytoplasm or totally outside or stuck at the cell membrane interface region. Hence, the N-termini of the peptides were labeled with 5(6)carboxyfluorescein by non-microwave-assisted manual SPPS coupling strategy and were purified with semi-preperative C18 columns at RP-HPLC.

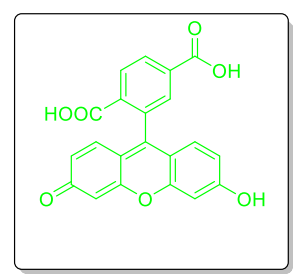

5(6)-Carboxy fluorescein
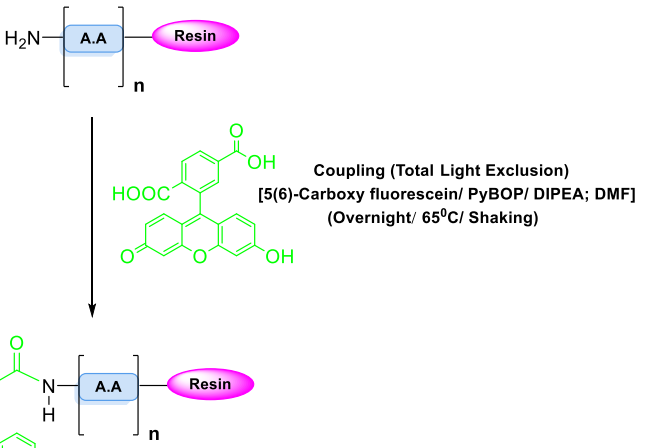

Cleavage (1.5 H/RT/ Shaking)

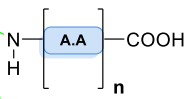

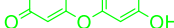

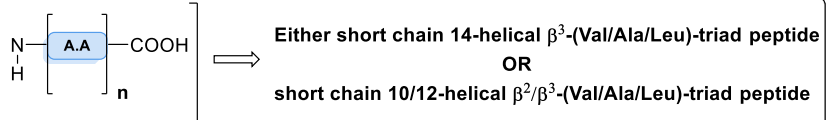

Figure 4.11: Schematic representation of the attachment of 5(6)-carboxyfluorescein to the solid supported short chain $\beta$-peptides to synthesize fluorescently labeled peptide derivatives

\subsection{Synthesized 6-Peptide: At-A-Glance}

\begin{tabular}{|c|c|}
\hline$\beta$-Peptide Sequences & $\begin{array}{c}\text { Assigned } \\
\text { Names }\end{array}$ \\
\hline HO- $\beta^{3} \mathrm{hV}-\beta^{3 \mathrm{~h} W}-\left(\beta^{3 \mathrm{~h}} \mathrm{~A}-\beta^{3} \mathrm{~h} \mathrm{~L}-\beta^{3 \mathrm{~h} V}\right)_{3-}-\beta^{3 \mathrm{~h}} \mathrm{~A}-\mathrm{NH}_{2}$ & $\mathbf{P 1}$ \\
\hline HO- $\beta^{3 \mathrm{hV}}-\beta^{3 \mathrm{~h}} \mathrm{~W}-\beta^{3 \mathrm{~h}} \mathrm{~L}-\beta^{2 \mathrm{~h}} \mathrm{~A}-\beta^{3 \mathrm{~h} V}-\beta^{2 \mathrm{~h}} \mathrm{~L}-\beta^{3 \mathrm{~h}} \mathrm{~A}-\beta^{2 \mathrm{hV}}-\beta^{3 \mathrm{~h}} \mathrm{~L}-\beta^{2 \mathrm{~h}} \mathrm{~A}-\mathrm{NH}{ }_{2}$ & $\mathbf{P 2}$ \\
\hline $\mathrm{HO}-\beta^{2 \mathrm{~h} V-} \beta^{3 \mathrm{~h}} \mathrm{~W}-\beta^{3 \mathrm{~h}} \mathrm{~L}-\beta^{2 \mathrm{~h}} \mathrm{~A}-\beta^{3 \mathrm{~h} V}-\beta^{2 \mathrm{~h} L-}-\beta^{3 \mathrm{~h}} \mathrm{~A}-\beta^{2 \mathrm{hV}-} \beta^{3 \mathrm{~h} L-}-\beta^{2 \mathrm{~h}} \mathrm{~A}-\mathrm{NH}{ }_{2}$ & P3 \\
\hline HO- $\left(\beta^{3 \mathrm{hV}}-\beta^{3 \mathrm{~h}} \mathrm{~A}-\beta^{3 \mathrm{~h}} \mathrm{~L}\right)_{2}-\mathrm{NH}_{2}$ & $\mathbf{P 8}$ \\
\hline HO- $\left(\beta^{3 \mathrm{hV}}-\beta^{3 \mathrm{~h}} \mathrm{~A}-\beta^{3 \mathrm{~h}} \mathrm{~L}\right)_{2}-\mathrm{FAM}$ & P9 \\
\hline
\end{tabular}




\begin{tabular}{|c|c|}
\hline $\mathrm{HO}-\beta^{2 \mathrm{~h} L-}-\beta^{3 \mathrm{~h}} \mathrm{~A}-\beta^{2 \mathrm{hV}}-\beta^{3 \mathrm{~h} L-}-\beta^{2 \mathrm{~h}} \mathrm{~A}-\beta^{3 \mathrm{hV}-\mathrm{NH}_{2}}$ & P6 \\
\hline HO- $\beta^{2}$ hL- $\beta^{3 \mathrm{~h}} A-\beta^{2 \mathrm{~h}} V-\beta^{3 \mathrm{~h}} \mathrm{~L}-\beta^{2 \mathrm{~h}} \mathrm{~A}-\beta^{3 \mathrm{~h} V-F A M}$ & P7 \\
\hline HO- $\left(\beta^{3 \mathrm{~h} K}\right)_{2-}\left(\beta^{3} \mathrm{~h} W\right)_{2}-\left(\beta^{3 \mathrm{~h} V}\right)_{19-}\left(\beta^{3 \mathrm{~h} W}\right)_{2-}-\left(\beta^{3} \mathrm{hK}\right)_{2}-\mathrm{NH}_{2}$ & P4 \\
\hline HO- $\left(\beta^{3 \mathrm{hK}}\right)_{2-}\left(\beta^{3 \mathrm{~h} W}\right)_{2-}-\left(\beta^{3 \mathrm{hL}}\right)_{15}-\left(\beta^{3 \mathrm{~h} W}\right)_{2-}\left(\beta^{3 \mathrm{~h} K}\right)_{2}-\mathrm{NH}_{2}$ & P5 \\
\hline HO- $\left(\beta^{3 \mathrm{~h} K}\right)_{1-}\left(\beta^{3} \mathrm{hW}\right)_{1-}\left(\beta^{3 \mathrm{~h} V}\right)_{12-}\left(\beta^{3 \mathrm{~h} W}\right)_{1-}\left(\beta^{3} \mathrm{hK}\right)_{1}-\mathrm{NH}_{2}$ & P10 \\
\hline HO- $\left(\beta^{3 \mathrm{~h} K}\right)_{1-}\left(\beta^{3 \mathrm{~h}} W\right)_{1-}\left(\beta^{3 \mathrm{~h}} \mathrm{~L}\right)_{9-}\left(\beta^{3 \mathrm{~h} W}\right)_{1-}\left(\beta^{3 \mathrm{~h}} \mathrm{~K}\right)_{1}-\mathrm{NH}_{2}$ & P11 \\
\hline
\end{tabular}

Table 4.1: Synthesized $\beta$-pepties and their fluorescently labeled analogues at-a-glance

\section{Results and Interpretation}

\subsection{Fluorescence-Spectroscopy Analysis of the $\beta$-Peptides: Transmembrane Peptide Insertion into Lipid Bilayers}

Fluorescence spectroscopy has become a crucial tool in biochemical research due to its robustness and high sensitivity. The on-going development of advanced optics and electronics has aggrevated the use of fluorophores for most biochemical analysis in preference to expensive and hazardous to handle radioactive tracers. Fluorophores absorb light of a specific wavelength $\left(\lambda_{\mathrm{EX}}\right)$, and after a brief interval of time, known as the fluorescence lifetime $(\tau)$, energy is emitted at a longer and specific wavelength $\left(\lambda_{E M}\right)$. In general, the fluorescence study of biomolecules, e.g., lipids, oligosaccharides, oligonucleotides, proteins and membranes, needs prolonged fluorescent labeling processes. Large varieties of fluorescent molecules are commercially available, that include biological fluorophores (e.g., the green fluorescent protein), organic dyes (e.g., fluorescein) and fluorescent nanoparticles (e.g., quantum dots). The selection of a fluorophore primarily depends on the photophysical properties applicable for specific research purposes and detection techniques. Besides the major shortcomings of fluorescent molecules being photolabile causing irreversible degradation or photobleaching, another major experimental drawback is the tedious and time-consuming procedures for labeling of the targets. In order to simplify these 
challenges, using intrinsic fluorescence of tryptophan residues to detect several biophysical phenomena came into play.

The photophysical properties of Trp are highly sensitive to its surrounding microenvironment. The fluorescence of Trp was found to be strongly affected by the polarity of its surrounding microenvironment. In presence of a polar hydrophilic microenvironment, a strong red-shift could be observed in Trp-fluorescence maxima $\left(\lambda_{\max }\right)$ ranging from 305-355 $\mathrm{nm}$. [167] On the other hand, if the surrounding microenvironment changed to non-polar hydrophobic, a strong blue-shift was observed in that same range. This special sensitivity of Trp-fluorescence to its local environment was used to investigate the insertion of the synthesized $\beta$-peptides into specific lipid bilayers.

Due to the high fluorescence sensitivity of the Trp-residues to their local environments, the Trpresidues in proteins showed a fluorescence maximum ( $\lambda_{\max }$, blue shift) of about $305-325 \mathrm{~nm}$ whe the residue was surrounded by non-polar hydrophobic microenvironment, such as, lipids. With the increase in the polarity of the Trp-micro-environment, the red shift in $\lambda_{\text {max }}$ started taking place and at a moderately polar/non-polar amphipathic environment, such as in membranewater interface region, the $\lambda_{\max }$ was found to be in a range of 330-345 $\mathrm{nm}$. Moreover, when the Trp-local environment was polar, as in water or any other aqeous solution, the red shift of the Trp-fluorescence was at maximum with the $\lambda_{\max }$ value ranging between $248-355 \mathrm{~nm}$. Keeping the unique Trp-fluorescence sensitivity to its local environment in mind, each of the synthesized $\beta$-peptides contained at least one $\mathrm{D}$ - $\beta^{3}$-Trp-residues towards the $\mathrm{C}$-termini to aquire knowledge on the exact location of the D- $\beta^{3}$-Trp-residues in presence of the lipid membrane (Figure 5.1). 

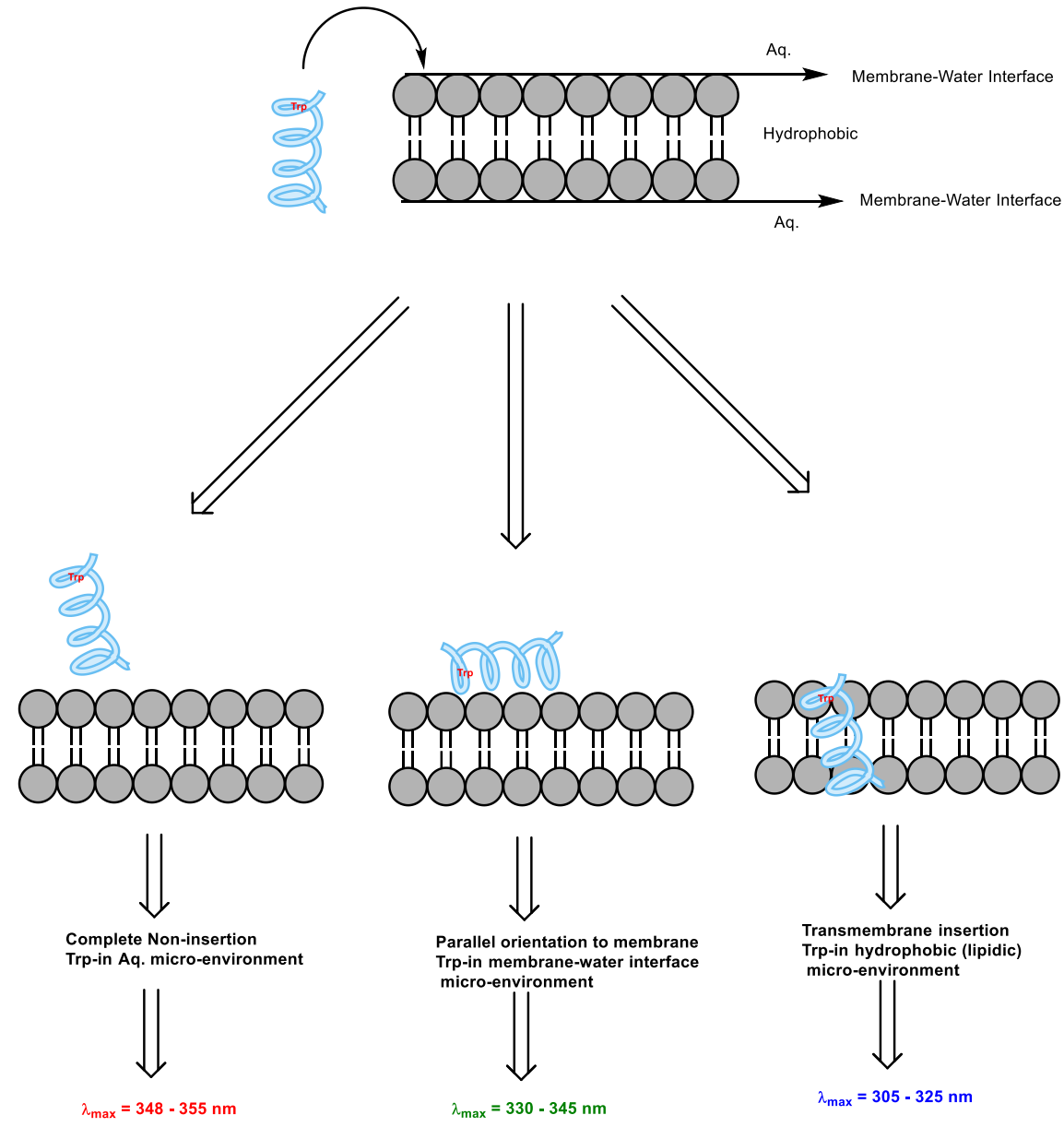

Figure 5.1: Schematic illustration for detection of peptide orientation into lipid membranes based on intrinsic Trp-fluorescence

The suitable match between the hydrophobic core length of the peptides and the bilayer thickness of the lipid membrane was one of the main factors towards effective transmembrane insertion and spanning of the peptides through the bilayer. The peptides, P1, P2, P3, P10 and P11 had comparable hydrophobic core length of around 18-19 Å. Therefore, DLPC lipid membrane was chosen to investigate on the transmembrane insertion of this peptide, as the bilayer thickness of DLPC almost matched with that of the peptides. 


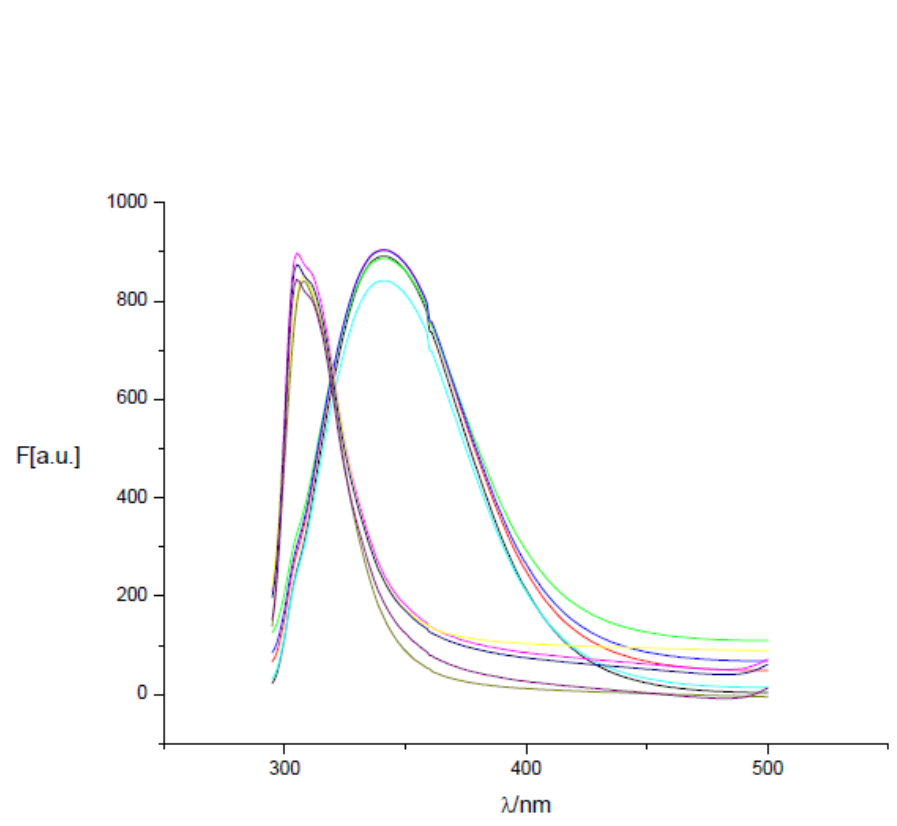

Figure 5.2: Trp-Fluorescence spectra of peptides, P1, P2, P3, P10 and P11 in solution (TRIS buffer) and in DLPC large unilamellar vesicles (LUV) with P:L ratio constant at 1:50, peptide concentration $12 \mu \mathrm{M}$ and temperature $20^{\circ} \mathrm{C}$.

From the Figure-5.2, itcould be observed that in solution (TRIS buffer), the $\lambda_{\max }$ of Trpfluorescence were in the regions of 348-352 $\mathrm{nm}$ indicating an aqueous polar surrounding of the Trp-residue at the C-termini. So, presence of the peptides in TRIS buffer was confirmed. On the other hand, after incubating the peptides with the large unilamellar vesicles of DLPC, the $\lambda_{\max }$ of all the peptides exhibited a distinctive blue shift to a range of 305-308 $\mathrm{nm}$. This blue shift at the intrinsic Trp-fluorescence confirmed that the Trp-residues at the C-termini of the peptides was in a hydrophobic (lipidic) microenvironment. This verified the transmembrane insertion and spanning of all these peptides in DLPC with almost perfect hydrophobic matching scenario. Therefore, the initial objective towards verification of the transmembrane insertion and spanning of the synthesized $\beta$-peptides, including the alternate 10/12-helical peptide, into selected lipid membranes was accomplished and confirmed by intrinsic Trp-fluorescence measurements.

After confirming the successful transmembrane insertion of the synthesized $\beta$-peptides into DLPC lipid membrane, it was time to focus on the second objective to shed light on the effect of helical macro-dipole moment on transmembrane insertion and membrane spanning of the $\beta$ peptides. This target was accomplished by estimation of the quantitative compensation of negative hydrophobic mismatch between the different target peptides and a group of lipid 
membranes using varying concentrations of EtOH. The negative hydrophobic mismatch between transmembrane proteins and lipids has already been discussed in section-1.5 and the mechanism of compensation of the negative hydrophobic mismatch by reduring the membtane bilayer thickness using EtOH has also been briefly addressed in section-1.6. The idea was to first take a group of synthesized $\beta$-peptides with different secondary helical structures, composed of similar amino acid sequences and of comparable hydrophobic core lengths. So, the peptides, P1 [14-helix, (-)-helical macro-dipole, $\beta^{3}$-(Val/Ala/Leu)-triad], P2 [10/12-helix, Zero-helical macrodipole, $\quad \beta^{3} / \beta^{2}$-(Val/Ala/Leu)-triad], P3 [10/12-helix, Zero-helical macro-dipole, $\beta^{2} / \beta^{3}$ (Val/Ala/Leu)-triad], P10 [14-helix, (-)-helical macro-dipole, $\beta^{3}$-(Val)] and P11 [12-helix, (-)helical macro-dipole, $\beta^{3}$-(Leu)] were selected. All these peptides had a comparable hydrophobic core length of about 18-19 $\AA$ and peptides P1, P2, P3 were composed of triad sequences (Val/Ala/Leu) while the hydrophobic cores of P10, P11 were made of only $\beta^{3}$-(Val) and $\beta^{3}$-(Leu), respectively. All these peptides had a $\beta^{3}$-(Trp)-residue attached towards the C-termini. A set of three lipids were chosen, DLPC, DMPC and POPC for these peptides. These lipids were structurally not very different from each other, so the results with these lipids should be comparable to each other.
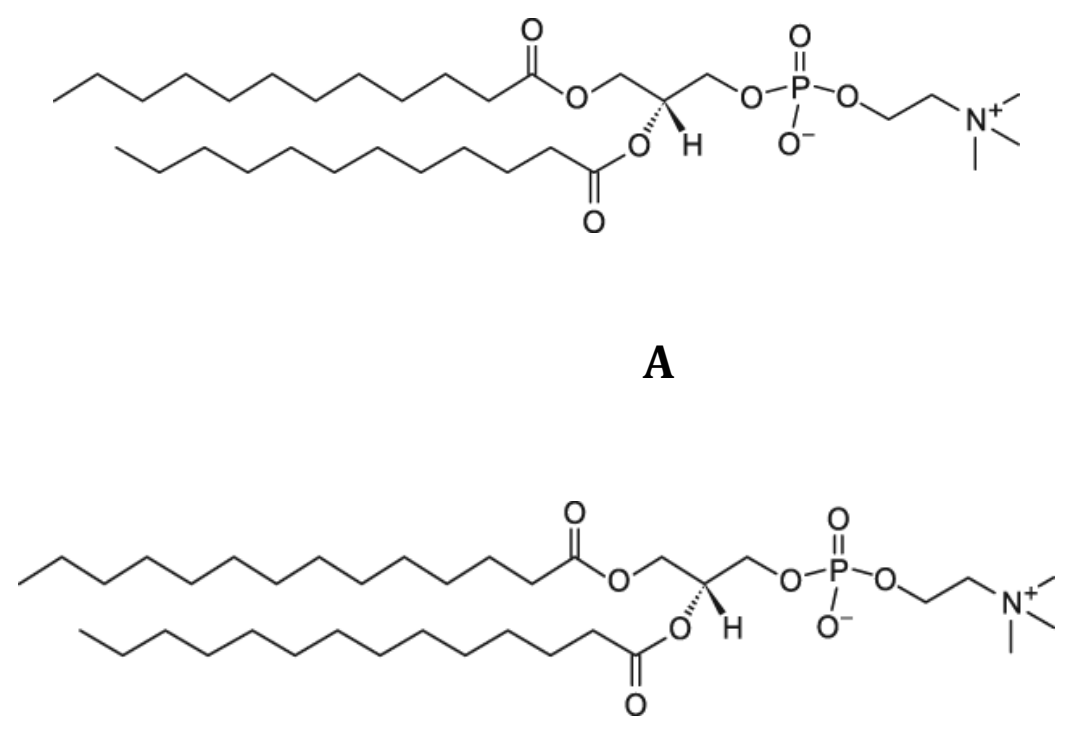

B

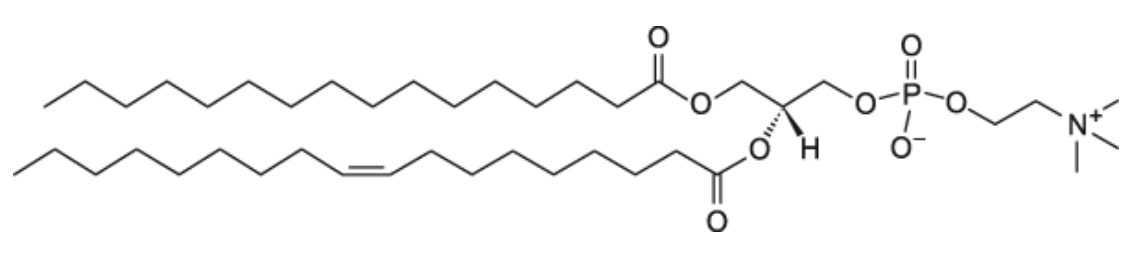


Figure 5.3: Chemical structures of the three chosen lipids, DLPC $(\boldsymbol{A}), D M P C(\boldsymbol{B})$ and $P O P C(\boldsymbol{C})$

The lipis were chosen in such a way that the bilayer thickness of DLPC, being the thinnest one, almost perfectly matched with that of the peptides. Gradually, the bilayer thicknesses of DMPC and POPC got incresed such a way that a negative hydrophobic mismatch occured between the peptides and these lipids with an extent of 6-7 $\AA$ to 9-10 $\AA$, rspectively. According to previous reports, the peptide-lipid system can compensate the negative hydrophobic mismatch upto 3-4 $\AA$ and the peptide could be inserted into the membrane. But if the negative mismatch was higher than this range, there was a large possibility that either the peptide would orient itself parallel to the membrane and stick to the membrane-water interface region or would not get inserted at all and remain in the aqueous region only. $[45,50]$ So, the peptides were expectected to get inserted into the DLPC lipid bilayer due to favourable hydrophobic matching, but in case of DMPC and POPC, the mismatch should be large enough and membrane insertion was not expected. In order to minimize the hydrophobic mismatch, $\mathrm{EtOH}$ was used to reduce the bilayer thicknesses of the lipid membranes, so that by compensation of hydrophobic mismatch transmembrane insertion of the peptides could be possible. Now the peptides in the selected group differed to each other mainly in their secondary helical structures, which corresponded to the helical macro-dipole moment. So, the minimum concentration of EtOH for minimum compensation of hydrophobic mismatch required for successful insertion of each types of synthesized peptides into each lipid membrane was monitored carefully. This gave an indication that the higher the minimum concentration of EtOH was needed, the more compensation of the hydrophobic mismatch was required to successfully insert a peptide with a specific secondary structure into the specific lipid bilayer. It in turn implied that the more the mismatch comensation by EtOH was required, the more difficult it was for the peptide to be inserted and spanned through the lipid bilayer system. Hence, if a difference in ease of transmembrane insertion between peptides with similar hydrophobic core length and different secondary helical structures into a specific lipid bilayer system could be detected by the strategy that would indicate an effect of the secondary helical structures of the peptides towards transmembrane insertion and spanning. In this case, one of the major distinctive difference between the various secondary structures being the helical macro-dipole moment, such an obserbvation would definitely verify possible role of the helical macro-dipole moment in transmembrane insertion and spanning of the $\beta$-peptides. 


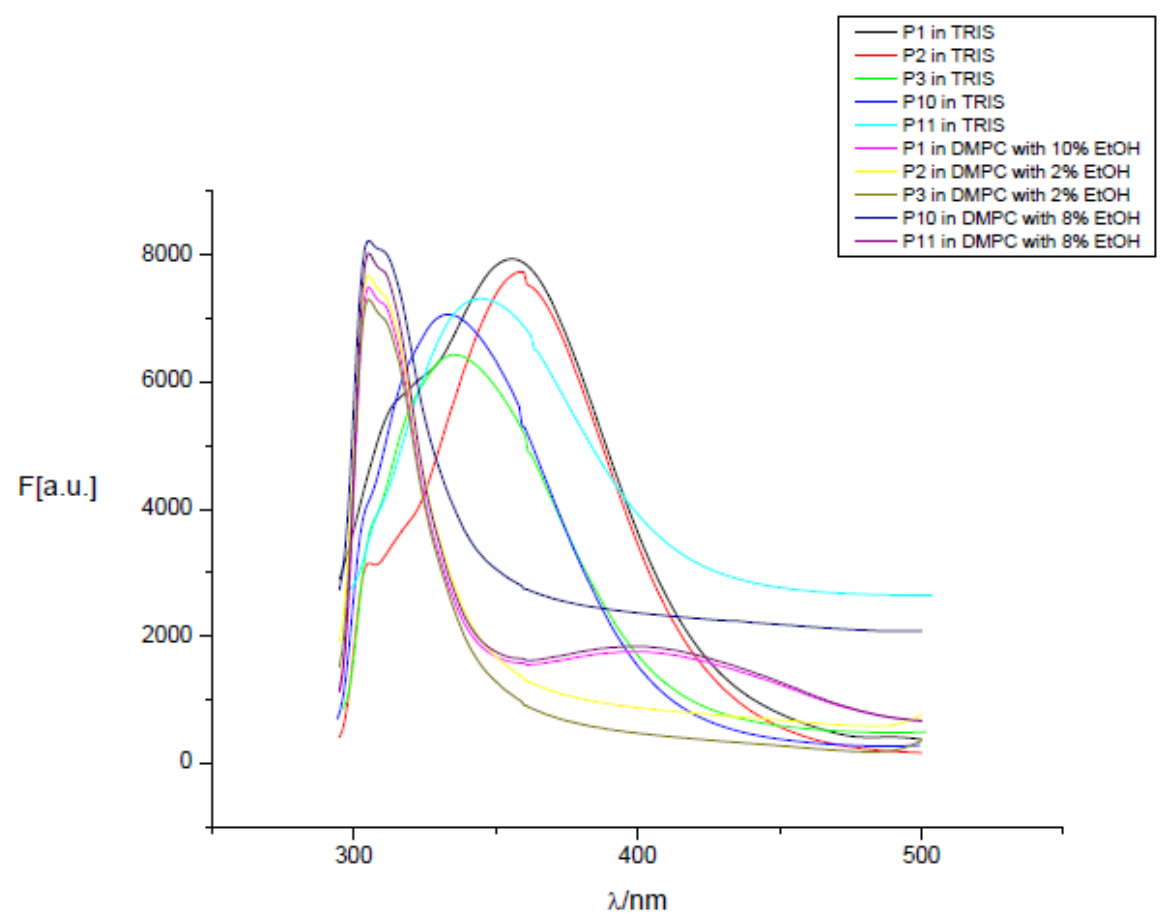

A

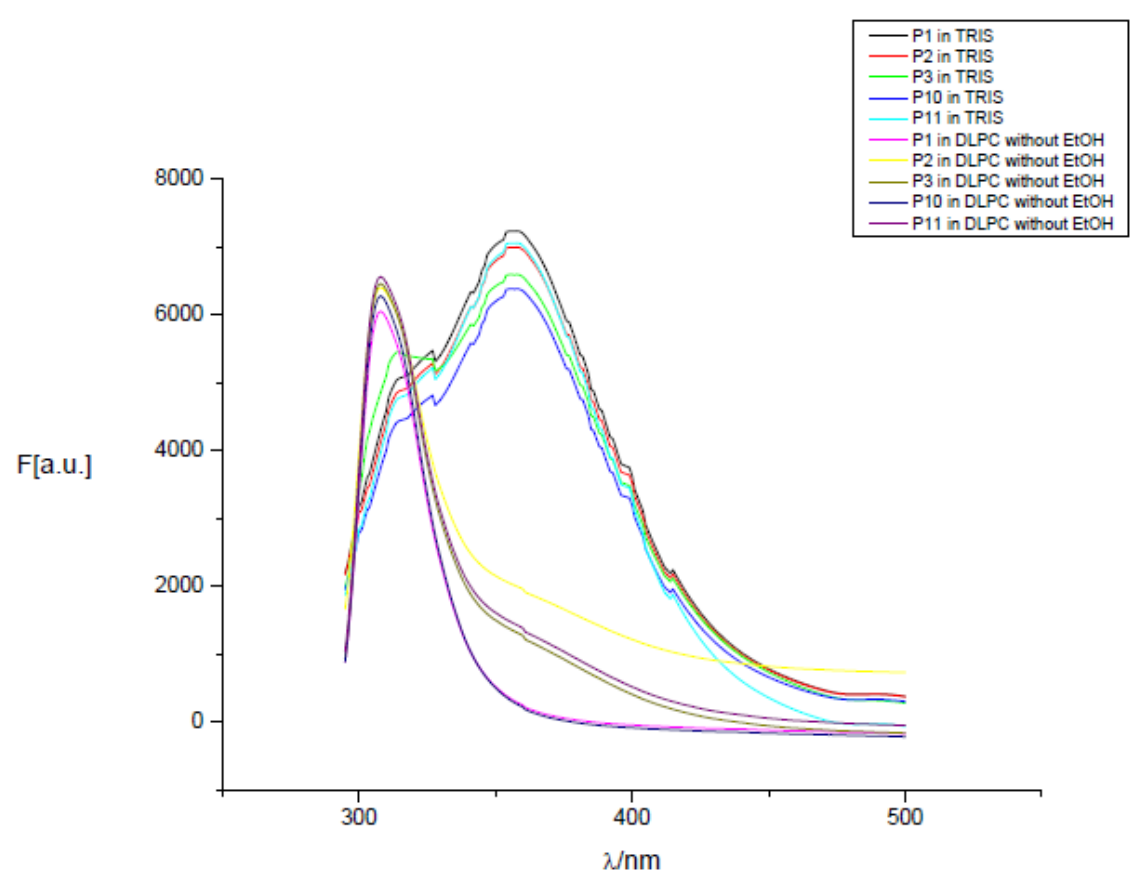

B 


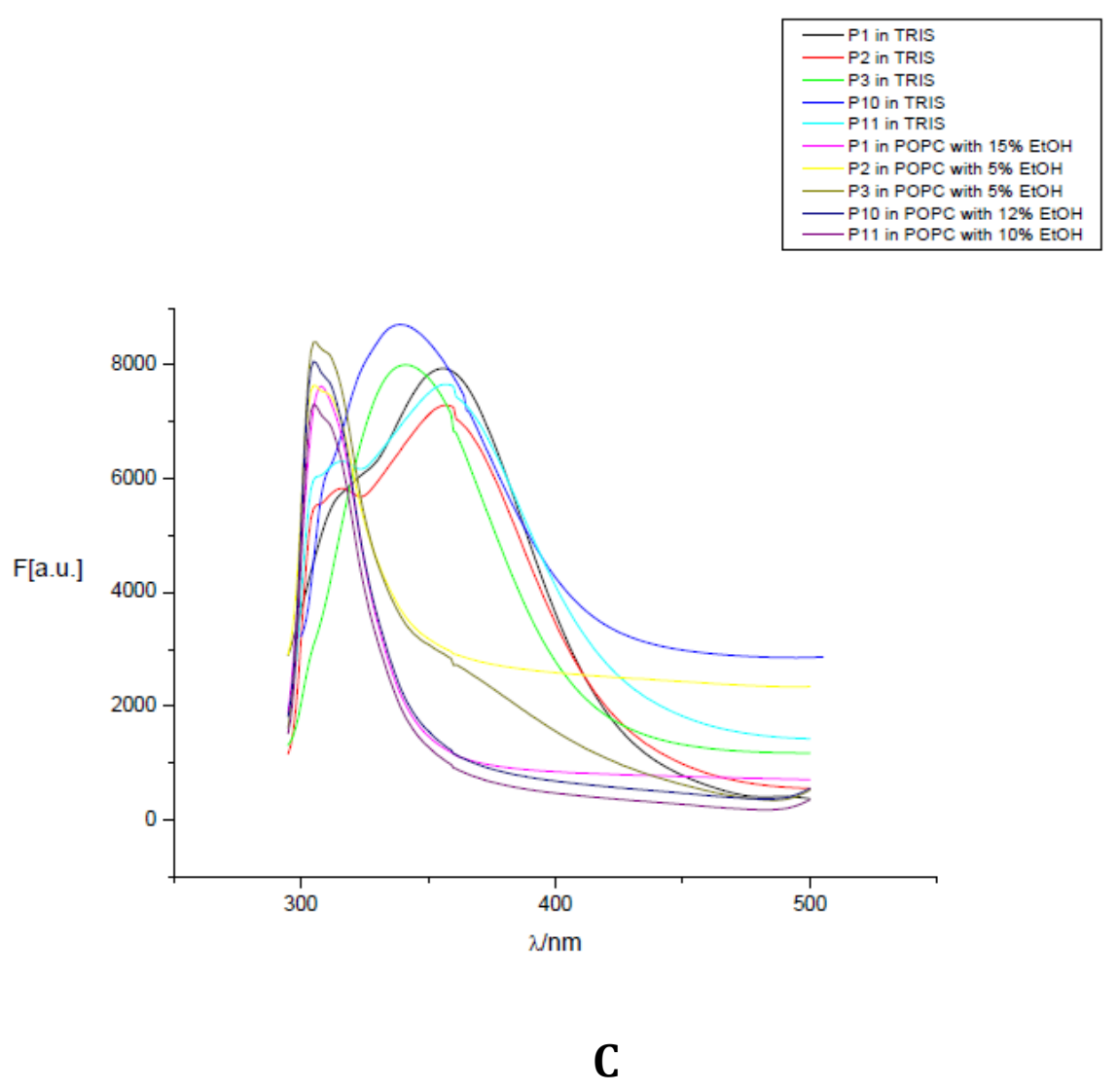

Figure 5.4: Comparative fluorescence spectra of $\boldsymbol{P 1}, \boldsymbol{P 2}, \boldsymbol{P} 3, \boldsymbol{P} 10$ and $\boldsymbol{P 1 1}$ in TRIS buffer and in three different lipid bilayers, DLPC (A), DMPC $(\boldsymbol{B})$ and POPC $(\boldsymbol{C})$, using constant P:L ration 1:50, peptide concentration $12 \mu \mathrm{M}, 50^{\circ} \mathrm{C}$ temperature and different minimum concentrations of EtOH for transmembrane insertion of the peptides via compensation of negative hydrophobic mismatch

From the Figure-5.4 (A), it was evident that due to proper hydrophobic match between the hydrophobic cores lenghths of the peptides and the bilayer thickness of DLPC, all the pepides were inserted into DLPC unilamellar vesicle without using EtOH. The negative hydrophobic mismatch between the peptides and DMPC was about 5-7 A. Since, none of the peptides could be inserted naturally due to this higher mismatch, EtOH was used to reduce the bilayer thickness of DMPC to compensate the negative hydrophobic mismatch. But it was observed that different peptides required different minimum concentration of EtOH to suceessfully insert the peptides into DMPC bilayer. The 14-helical peptides, P1 and P10 along with the 12-helical peptide, P11, required 10\%, 8\% and $8 \% \mathrm{EtOH}$, respectively, to insert the peptides into the DMPC bilayer. On the other hand, the alternate 10/12-helical peptides, P2 and P3, required only 2\% EtOH for the insertion. So, there was a clear difference in requirement for compensation of negative hydrophobic mismatch between the 10/12-helical petides with zero macro-dipole moment and the 14- or 12-helical peptides with significant helical macro-dipole moments. The 10/12-helical 
peptides required much less minimum concentration of EtOH, that is, less compensation of mismatch was required to successfuly insert the peptides into the DMPC bilaer. While the 14and 12-helical peptides required much higher minimum concentration of EtOH, that is, more compensation of mismatch was required to successfully insert the peptides into DMPC bilayer. Similarly, to get introduced into POPC bilayer, the 10/12-helical peptides, P2, P3, needed only 5\% minimum concentration of EtOH while the 14-helical peptides, P1, P10 and 12-helical peptide, P11, required 10\%, 12\% and $12 \%$ of minimum concentration of EtOH, respectively. In case of POPC, the negative hydrophobic mismatch was even higher of about 8-10 ̊. So, as expected, higher minimum concentration of EtOH was needed to compensate the negative mismatch in higher extent for successful transmembrane insertion of the peptides into POPC bilayer. In analogy to DMPC, the 10/12-helical peptides needed much lower minimum concentration of $\mathrm{EtOH}$, that is, less compensation of negative mismatch to insert the peptides, whereas the 14- or 12-helical peptides required much higher minimum concentration of EtOH, that is, higher extent of negative mismatch compensation was needed to successfully introduce the peptides into POPC bilayer.

From this experimental observation, it could well be assumed that one of the major differences between the 10/12-heliclal peptides and the 14- or 12-helical peptides, being the absence and presence of helical macrodipole moment, respectively, the latter could have a significant effect on transmembrane introduction and spanning of the peptides to lipid bilayers. In order look for a justified explanation towards this experimental observation, the secondary structures of the different peptides were one again cultivated. It was already discussed that the hydrophobic side chains of the 10/12-helical peptides in their secondary structure were distributed covering a higher surface area, while that of the 14-helical peptides were distributed more evenly in three faces covering less surface area. This made the 10/12-helical peptides more hydrophobic in nature. On the other hand, the lack of helical macro-dipole moment made the secondary structure of 10/12-helical peptides more apolar or hydrophobic in nature. But the 14- or 12helical peptides possessed considerable helical macro-dipole moment that introduced partial charged characters at the two termini in the secondary helical structures of these peptides and this charged character made the overall structure less hydrophobic. 


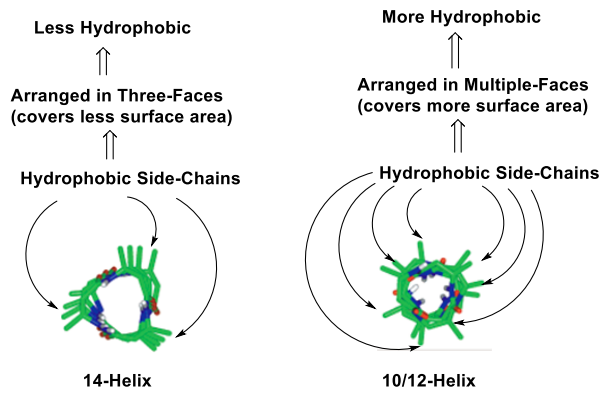

A

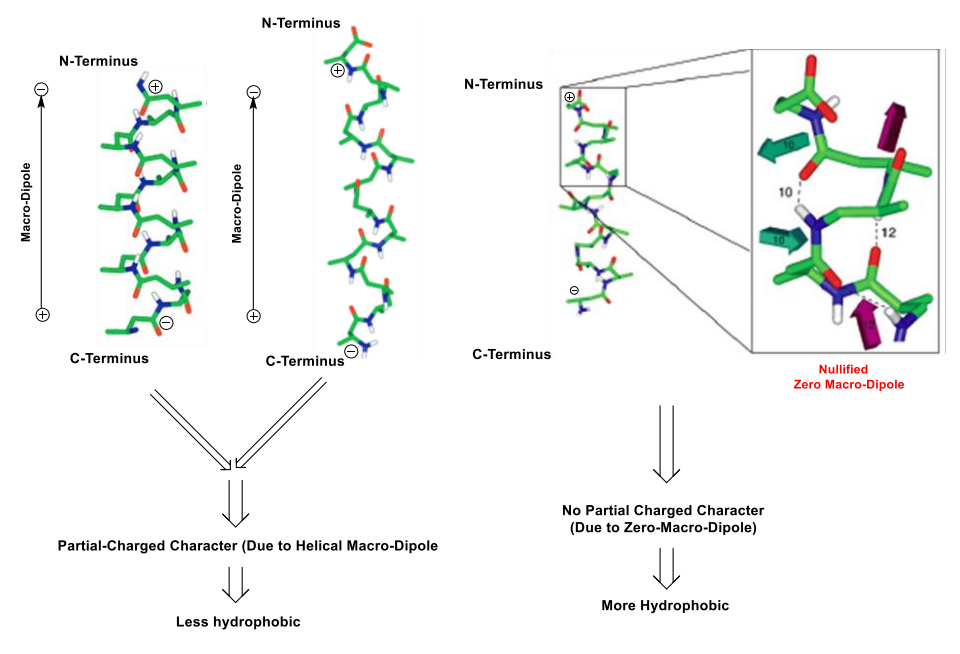

B

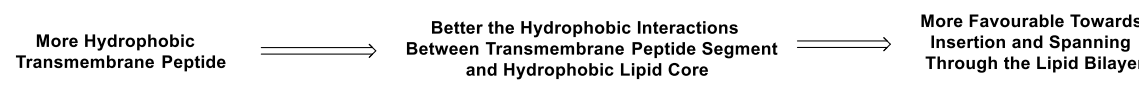

c

Figure 5.5: Comparative schematic illustration towards the difference in hydrophobic character of 14-helix and 10/12-helical $\beta$-peptides due to distribution of side chains (A) and helical macrodipole moment (B); Concludingrelationship between the hydrophobic character of the transmembrane peptides and the propensity towards membrane insertion and spanning (C)

Since, the inner part of the lipid bilayers was completely hydrophobic in nature, so the more the hydrophobic character of the secondary helical structures of the peptide, the more favourable it was to get inserted and spanned through the lipid membrane due to energetically favourable hydrophobic interactions between the transembrane peptide segment and the fatty acid chains in the lipid core. So, the 10/12-helical peptides being more hydrophobic in nature, the insertion and spanning of these pepides should be more favourable than that of the 14- or 12-helical peptides. In addition, this assumption was properly supported by the experimental results depicted in Figure-5.4. Therefore, it could be concluded that the helical macro-dipole moment 
could have a major role in transmembrane insertion and spanning of membrane peptides into lipid bilayers. The more the overall helical macro-dipole moment of the peptide, the more the hydrophobic character of the peptide and the more favourable it was to insert and span the peptides through the lipid membranes. Therefore, the 10/12-helical peptdes with zero macrodipole moment were more suitable as $\beta$-peptide based model transmembrane domain systems and the nullified macro-dipole moment in its secondary helical structure was the major advantage behind this conclusion (Figure 5.5).

As discussed previously, as the overall helical macro-dipole moment was the summation of the individual dipole moment of the amide bonds of each amino acid residues, so the overall helical macro-dipole moment of transmembrane peptides increased with the elongation of peptide chain lenghth. To make sure that the macro-dipole indeed influenced the transmembrane insertion and spanning of the peptides indeed, two more peptides, a 14-helical (P4) and a 12helical (P5), of longer transmembrane core length of about 27-29 $\AA$ and $30 \AA$, respectively, were investigated by the same strategy via estimation of the minimum compensation of negative hydrophobobic mismatch required using $\mathrm{EtOH}$ to successfully insert into a class of three different lipids. As the length of the hydrophobic cores of the peptides were much longer, so a different group of three lipids, POPC, 22:1(Cis)PC and 24:1(Cis)PC, were used. The bilayer thickness of POPC (28-29 $\AA$ ) almost matched with the transmembrane lengths of the peptides [166] and the negative hydrophobic mismatch gradually increased from 22:1(Cis)PC [34-35 ̊̊] to 24:1(Cis)PC [37.5-38 $\mathrm{A}$ ] to an extent of $7 \AA, 10 \AA$ and $5 \AA$, $8 \AA$ for the peptides P4 and P5, respectively.

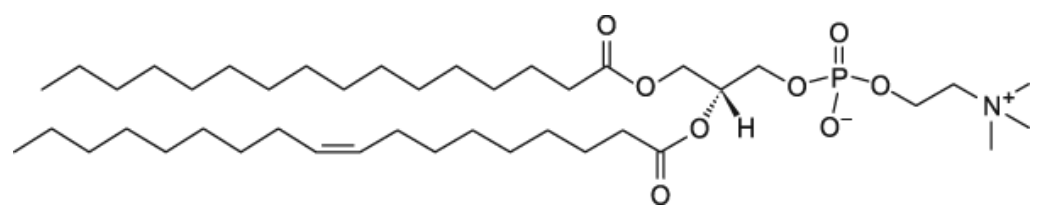

A

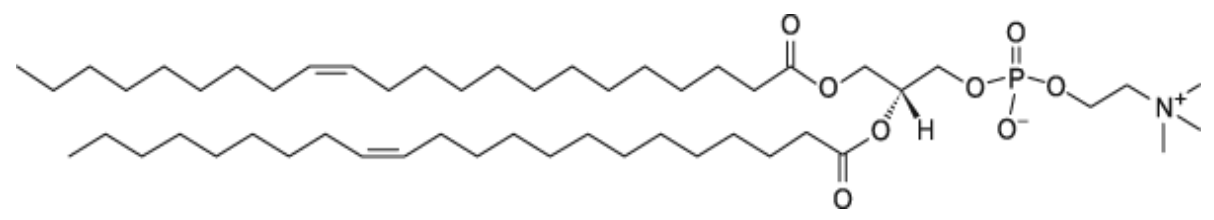




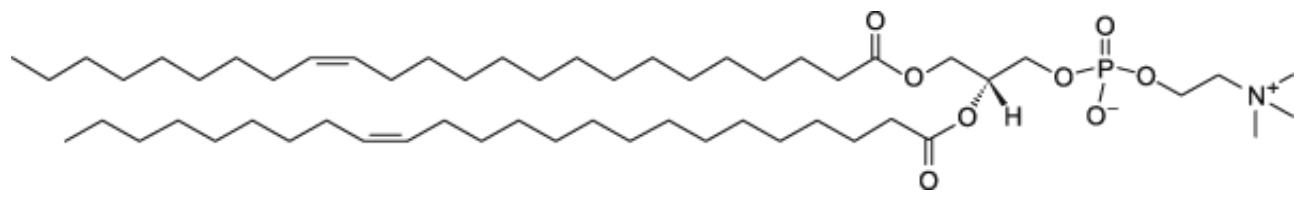

C

Figure 5.6: Chemical structures of the three chosen lipids, POPC (A), 22:1(Cis) PC (B) and 24:1(Cis) $P C(C)$

So the peptide and lipid systems were selected in such a way that the gradual increase in negative hydrophobic mismatches reflected the case with peptides, P10 (14-helical) and P11 (12-helical), against the DLPC. DMPC, POPC lipid systems. The only differences between these two different peptide-lipid systems were the transmembrane lenghths of the peptides that corresponded to the change in increased helical macro-dipole moment along with the increase in transmembrane peptide chain lengths. The hydrophobic core of peptides P4 and P10 were composed with only D- $\beta^{3}$-Val while that of P5 and P11 were with only D- $\beta^{3}$-Leu residues. Since the negative mismatch between P10-DMPC corresponded approximately to that of P422:1(Cis)PC, P10-POPC to P4-24:1(Cis)PC, P11-DMPC to P5-22:1(Cis)PC and P11-P0PC to P524:1(Cis)PC, so any difference in the minimum concentration of EtOH required to introduce the peptides between the systems P10-DMPC/ P4-22:1(Cis)PC, P10-POPC/P4-24:1(Cis)PC, P11DMPC/P5-22:1(Cis)PC and P11-POPC/ P5-24:1(Cis)PC, would verify that it was indeed the length of the transmembranepeptide segments, connected to the magnitude of helical macrodipole moment, that acted as one of the regulatory parameters for transmembrane peptide insertion and spanning into lipid bilayers.

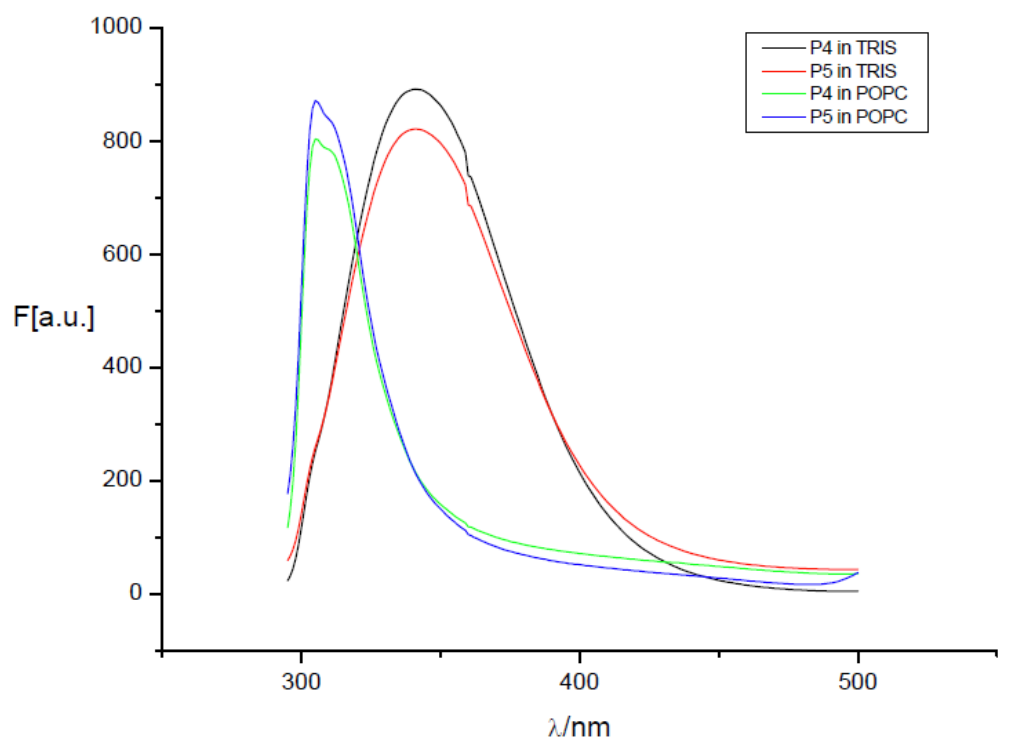


A

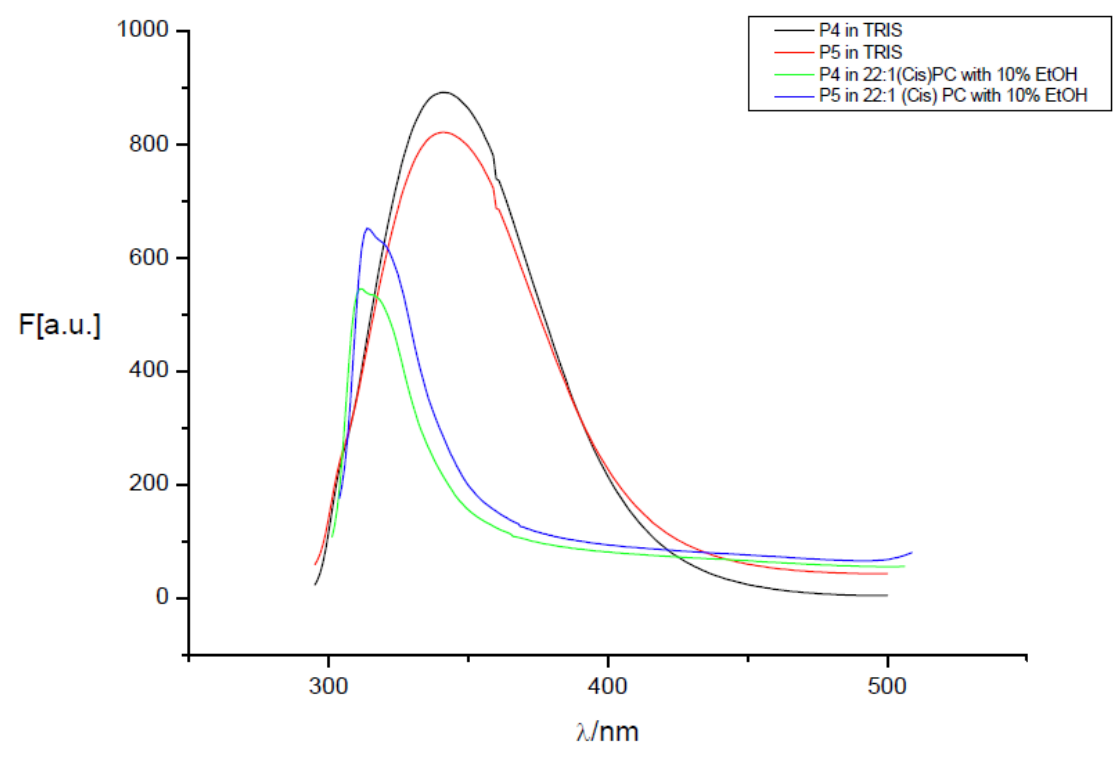

B

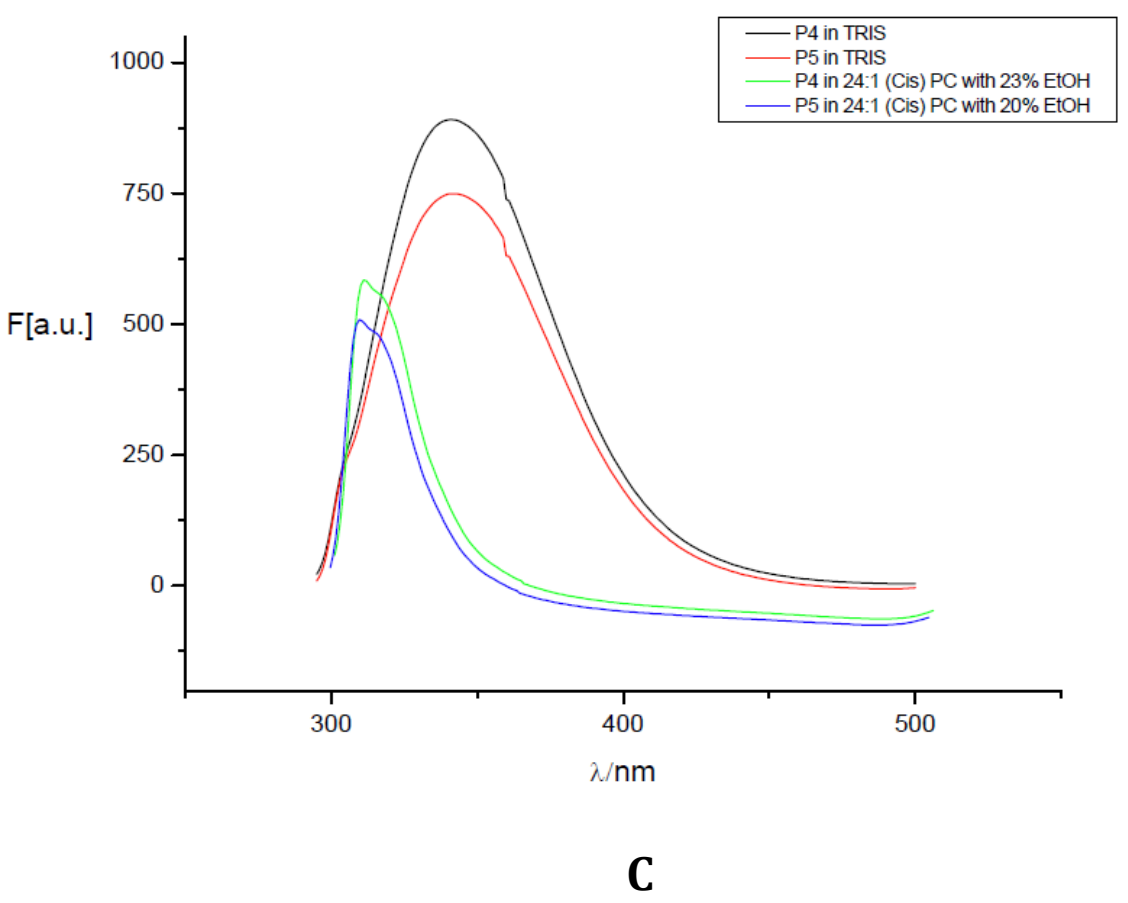

Figure 5.7: Comparative fluorescence spectra of $\mathbf{P 4}$ and $\mathbf{P 5}$ in TRIS buffer and in three different lipid bilayers, POPC (A), 22:1(Cis)PC (B) and 24:1(Cis)PC (C), using constant P:L 1:50, peptide concentration $12 \mu \mathrm{M}$, temperature $50^{\circ} \mathrm{C}$ and different minimum concentrations of EtOH for transmembrane insertion of the peptides via compensation of negative hydrophobic mismatch 


\begin{tabular}{|c|c|c|c|c|}
\hline $\begin{array}{c}\text { A. 14-Helical } \\
\text { Peptides }\end{array}$ & \multicolumn{2}{|c|}{ P10/DMPC -P4/22:1(Cis)PC } & \multicolumn{2}{|c|}{ P10/POPC- P4/24:1(Cis)PC } \\
\hline $\begin{array}{c}\text { (-)-Hydrophobic } \\
\text { Mismatch }\end{array}$ & $6-7 \AA$ & $5-6 \AA$ & $10-11 \AA$ & $9-10 \AA$ \\
\hline $\begin{array}{c}\text { Min.Conc }{ }^{\mathrm{n}} \text { EtoH for } \\
\text { transmembrane } \\
\text { peptie insertion }\end{array}$ & $8 \%$ & $10 \%$ & $12 \%$ & $23 \%$ \\
\hline
\end{tabular}

\begin{tabular}{|c|c|c|c|c|}
\hline $\begin{array}{c}\text { B. 12-Helical } \\
\text { Peptides }\end{array}$ & \multicolumn{2}{|c|}{ P11/DMPC -P5/22:1(Cis)PC } & \multicolumn{2}{|c|}{ P10/POPC- P5/24:1(Cis)PC } \\
\hline $\begin{array}{c}\text { (-)-Hydrophobic } \\
\text { Mismatch }\end{array}$ & 5-6 Å & 4-5 $\AA$ & 9-10 & $7-8 \AA$ \\
\hline $\begin{array}{l}\text { Min.Conc }{ }^{\mathrm{n}} \text { EtoH for } \\
\text { transmembrane } \\
\text { peptie insertion }\end{array}$ & $8 \%$ & $10 \%$ & $10 \%$ & $20 \%$ \\
\hline
\end{tabular}

Table. 5.1: Tabular illustration of a comparison of the negative hydrophobic mismatch and the minimum concentration of EtOH required for transmembrane peptide insertion between the 14helical peptides, P4, P10 (A) and 12-helical peptide, P5, P11 (B)

It was evident from the comparative tabular descriptions exhibited in Table-5.1 (A), although the negative mismatch was greater in P10/DMPC system than that in P4/22:1(Cis) PC system, the latter required higher minimum concentration of $\mathrm{EtOH}$ to compensate the lower negative mismatch for successful insertion. Similarly, in P10/POPC the negative mismatch was higher than that in P10/24:1(Cis) PC system, but the latter required much higher minimum concentration of EtOH for the compensation of lower negative mismatch for efficient transmembrane insertion. As the difference between the two comparing peptides was in their chain lengths, which related to their overall helical macro-sipole moment, so it could be concluded that for 14-helical peptides, the longer the peptide chain length, the higher was the overall helical macro-dipole moment and the higher the macro-dipole moment in the peptide, 
the more was the charged character imposed into the sequence, that is, the less hydrophobic it was in nature. As it was already discussed that with the increase in the hydrophobicity of the transmembrane sequence, the transmembrane insertion was more favourable due to hydrophobic interactions between the hydrophobic core of the peptide and the acyl chains co nstituting the lipid bilayer core. Therefore, the less hydrphobic the systhesized 14-helical transmembrane $\beta$-peptides were, the more difficult it was to be inserted into the lipid bilayer and hence the more negative mismatch compensation was required using higher minimum concentration of EtOH for comparatively lower mismatch.

In analogy to the observations for 14-helical peptides, the comparative studies for the 12-helical peptides also showed similar results that could be observed in Table-5.1(B). Although the negative mismatch between the P11/DMPC was higher than that of P5/22:1(Cis) PC, the latter required higher minimum concentration of EtOH for successful transmmebrane peptide insertion. On the other hand, with a relatively lower negative mismatch between the P5/24:1(Cis) PC than that of P11/POPC, the former required higher minimum concentration of EtOH to successfully be inserted into the lipid bilayers. Hence, the 12 -helical $\beta$-peptides also held the fact that with the increase in helical macro-dipole moment due to increased peptide chain length, the hydrophobic character of the peptide got reduce which in turn made it comparatively diffult to get inserted and spanned across the lipid bilayers and thus required higher compensation of the negative hydrophobic mismatch using higher minimum concentration of EtOH.

\subsection{CD-Spectroscopic Analysis of the $\beta$-Peptides}

\subsubsection{Fundamentals of CD-spectroscopy}

Circular dichroism (CD) is the difference in the absorbance of left-handed circularly polarized light (L-CPL) and right-handed circularly polarized light (R-CPL). It occurs when a molecule contains one or more chiral chromophores (light absorbing groups). The theoretical details on the principles of CD-spectroscopy has long been well established in several literature reviews and hence that is out of the scope of this work. [161-164] 


\section{Where $\lambda$ is the wavelength}

The phenomenon of circular dichroism is very sensitive to the secondary structure of polypeptides and proteins. So, the evaluation of the secondary structures of the target peptides by CD-spectoscopy was performed. In general, the CD-spectrum in the near UV region (320-250 $\mathrm{nm}$ ) provides information about the surrounding environments of the peptide side chains and the protein tertiary structure contents. On the otehr hand, CD-spectrum in the far UV-region (240-180 nm) that relates to the absorption of the amide chromophore gives information on the secondary helical structures of the peptides. Hence, the CD-spectroscopy proved to be an important tool to elucidate the actual secondary helical patterns of the target peptides both in solution as well as inside lipid membranes.

\subsubsection{Secondary structure of target $B$-peptides in solution}

The CD-spectroscopic investigations on the synthesized $\beta$-peptides were carried out initially in solution (TFE) to check on the characteristic secondary structures of the peptides. It has been well established that the right-handed 14-helix exhibit a maxima near 210-215 nm and a minima near 195-203 nm. Just the opposite happens for a left-handed 14-helix. The characteristic CDspectra for a right-handed 12-helix displays a maxima near 200-205 nm and two minima near 220 and $190 \mathrm{~nm} .[\mathbf{1 0 1}, \mathbf{1 0 3}, \mathbf{1 5 8}]$ On the other hand, the right-handed alternate 10/12-helix depicts only one characteristic maxima near 199-203 nm. Of course, the helix content largely controls the magnitude of the ellipticity $(\theta)$. It has also been observed that the more the peptide length the more the helical content and higher the intensity of the ellipticity.

The $\beta$-peptides were examined in 2,2,2-trifluoroethanol (TFE) solvent. The TFE has been known to induce the helical pattern to the native conformation of the peptides because the dielectric constant of TFE (1-2 approx.) resembles the interior of the peptides and proteins, which in turn favours the formation of intramolecular hydrogen bonds to induce the stable favourable secondary helical structures. Hence using TFE stabilizes the secondary helicity of the peptides. [165]

The secondary structures of all the synthesized peptides were examined by CD-spectroscopy in TFE. It has already been discussed in section-3 that the peptide P1, composed of D$\beta^{3}$ (Val/Ala/Leu)-triad, was theoreticall expected to fold into a right-handed 14-helical secondary 
structure. Since, all the $\beta^{3}$ amino acid building blocks have a D-conformation, so a right-handed helix should be formed. Now, after the CD-measurements have been carried out for P1 in TFE (peptide concentration - $30 \mu \mathrm{M}$ ), it showed a characteristic maxima at $212 \mathrm{~nm}$ and a minima at $194 \mathrm{~nm}$. Similarly, the D- $\beta^{3}(\mathrm{Val})$-peptides, $\mathbf{P 4}$ and P10, that also was theoretically expected to form a rigt-handed 14-helix, exhibited a maxima at $210 \mathrm{~nm}, 208 \mathrm{~nm}$ and a minima at $194 \mathrm{~nm}$ and $193 \mathrm{~nm}$, respectively. Therefore, it was confirmed by CD-spectroscopic measurements the peptides with transmembrane hydophobic core composed of either only D- $\beta^{3}($ Val) or D$\beta^{3}$ (Val/Ala/Leu)-triad, all fold into characteristic right handed 14-helices in TFE.

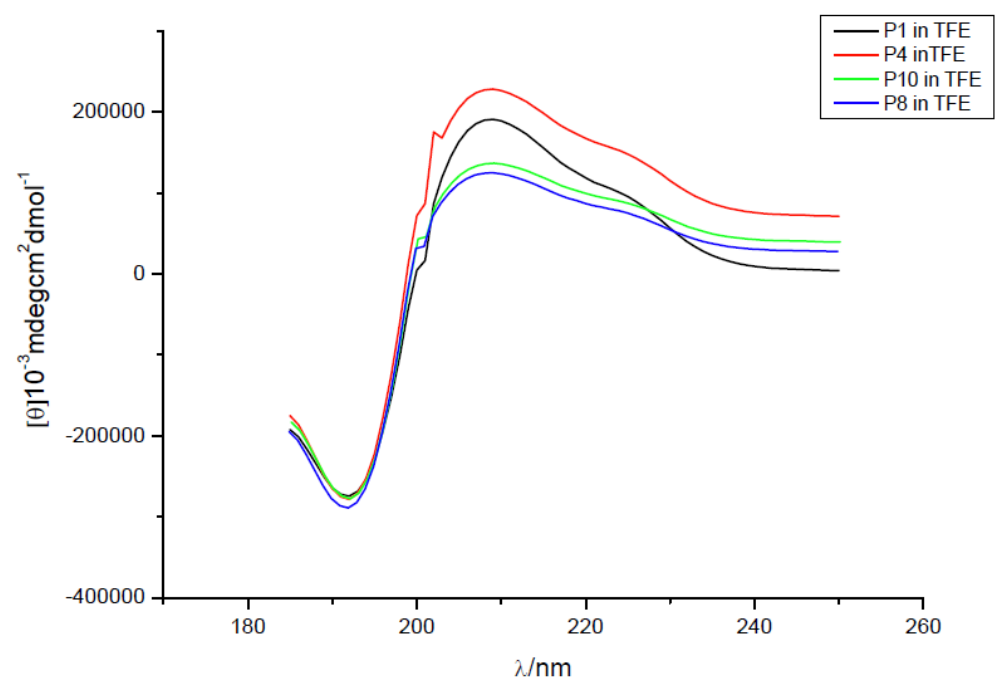

Figure 5.8: CD-spectroscopy plot for the three peptides, P1, P4, P8 and P10 exhibiting characeristic spectra patterns for right-handed 14-helical secondary structure

It can easily be noticed on Figure 5.8 that the ellipticities of P1, P8 and P10 are distinctivly less than that of P4. The P4 has a transmembrane hydrophobic core of maximum length (approx. 27$29 \AA$ ) while that of P1, P10 and P8 are approx. 18-19 $\AA, 18-19 \AA$ and $12 \AA$, respectively. So, this result established that although unlike $\alpha$-peptides, $\beta$-peptides did fold into stable 14 -helical secondary structures with as shorter sequence length as 6-amino acids (P8), but the ellipticity decrased gradually with the transmembrane core length. Another aspect that came into notice was although having the similar transmembrane peptide length, the ellipticity of peptide P1 was clearly higher than that of peptide P10 and that of P8 was almost same as P10, taking into account that the transmembrane length of $\mathbf{P 8}$ was much shorter than that of P10. This could only take place if the composition of the transmembrane peptide played a role in controlling the ellipticity, as the P1 and P8 were composed of D- $\beta^{3}-(\mathrm{Val} / \mathrm{ala} / \mathrm{Leu})$-triad while transmembrane 
segment of P10 was composed of $\mathrm{D}-\beta^{3}$-(Val) only. This indicated that the transmembrane sequence of $\mathrm{D}-\beta^{3}-(\mathrm{Val} / \mathrm{ala} / \mathrm{Leu})$-triad formed a more predominant 14-helical secondary structure with higher percentages of helical content compared to the peptides composed on D$\beta^{3}$-(Val)-oligomers.

On th eother hand, the peptides P5 and P11 with the transmembrane hydrophobic core composed of D- $\beta^{3}$ (Leu) were theoreticall expected to fold into left-handed 12-helices. The CDspectroscopic measurements were carried out on the peptides, P5 and P11, and the spectra displayed characteristic maxima at $203 \mathrm{~nm}$ and $204 \mathrm{~nm}$ as well as two minima at (218 nm, 219 $\mathrm{nm}$ ) and (192 nm, $191 \mathrm{~nm})$, respectively. These characteristic spectral patterns evidently showed that the two peptides, P5 and P11, folded into left-handed 12-helices in TFE.

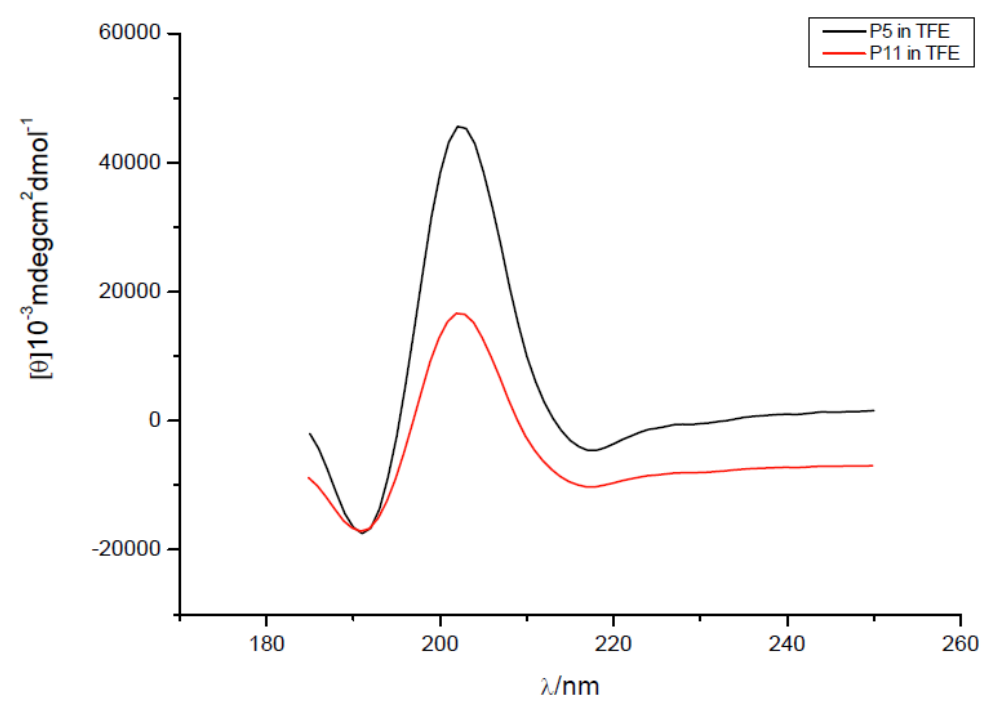

Figure 5.9: $C D$-spectroscopy plot for the two peptides, $P 5$ and $P 11$ exhibiting characeristic spectra patterns for left-handed 12-helical secondary structure

It is also quite evident from the Figure 5.9 that similar to the 14-helical peptides, the ellipticity or the helical content of the secondary structure evident depended on the transmembrane peptide length as the P5 with transmembrane core thickness of around 30-31 A has distinctive higher helical content that that of the shorter peptide, P11, with transmembrane core length of approx. 18-19 ̊. 
The primary target peptides, P2, P3 and P6, were also investigated with CD-spectroscopy in order to verify whether they actually folded into the rare alternate 10/12-helical secondary structure.

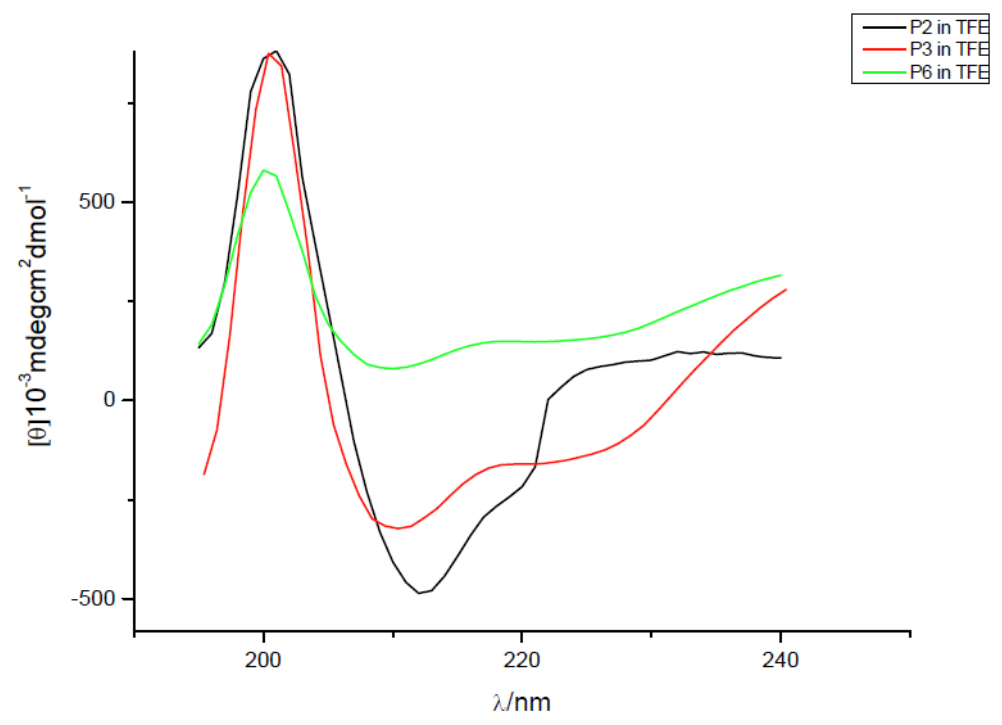

Figure 5.10: $C D$-spectroscopy plot for the three peptides, P2, P3 and $\mathbf{P 6}$ exhibiting characeristic spectra patterns for right-handed altrnate 10/12-helical secondary structure

The CD-spectral pattern for the three peptides were found to converge at a single common point of maxima between $200-202 \mathrm{~nm}$. This characteristic spectral pattern proved that all the three peptides did fold into right-handed alternate 10/12-helical secondary structure in TFE. It was alos evident from the spectra that the magnitude of ellipticity for P6 was much lower than that of P2 and P3. P2 and P3 peptides having transmembrane core length of approx. 18-19 ̊ had clearly higher helical content than the peptide $\mathbf{P 6}$ that was composed of only 6 -amino acids of alternate $\beta^{2} / \beta^{3}$-(Val/Ala/Leu)-triad with a length of around $12 \AA$. This also showed that only an oligomer of six- alternate $\beta^{2} / \beta^{3}$-amino acids could form stable 10/12-helical secondary structure in TFE.

\begin{tabular}{|l|c|c|c|c|}
\hline TFE/14-Helix & P1 & P4 & P8 & P10 \\
\hline Maxima & $208 \mathrm{~nm}$ & $206 \mathrm{~nm}$ & $209 \mathrm{~nm}$ & $211 \mathrm{~nm}$ \\
& & & & \\
\hline Minima & $193 \mathrm{~nm}$ & $195 \mathrm{~nm}$ & $191 \mathrm{~nm}$ & $194 \mathrm{~nm}$ \\
\hline
\end{tabular}


A

\begin{tabular}{|c|c|c|}
\hline TFE/12-Helix & P5 & P11 \\
\hline Maxima & $205 \mathrm{~nm}$ & $206 \mathrm{~nm}$ \\
\hline Minima & $219 \& 191 \mathrm{~nm}$ & $217 \& 193 \mathrm{~nm}$ \\
\hline
\end{tabular}

B

\begin{tabular}{|l|c|c|c|}
\hline TFE-10/12-Helix & P2 & P3 & P6 \\
\hline Maxima & $201 \mathrm{~nm}$ & $202 \mathrm{~nm}$ & $201.5 \mathrm{~nm}$ \\
\hline Minima & $213 \mathrm{~nm}$ & $210 \mathrm{~nm}$ & $212 \mathrm{~nm}$ \\
\hline
\end{tabular}

C

Table 5.2: CD-spectroscopy results of peptides, P1, P4, P8, P10 (A) P5, P11 (B) and P2, P3, P6 (C) in TFE at-a-glance

After the confirmation towards the secondary structures of the synthesized $\beta$-peptides in TFE from the results depicted in Table 5.2, the next target was to shed light on the stability of the 14helical and 10/12-helical transmembrane $\beta$-peptides and compare between these two classes to investigate on whether the peptide helical macrodipole moment has any role in stabilizing the secondary helical structures of the peptides. CD-spectroscopy of a 14-helical and a 10/12-helical $\beta$ peptides of comparable length and similar sequences were carried out at different temperatures, starting from $20^{\circ} \mathrm{C}$ to $80^{\circ} \mathrm{C}$ in TFE. 


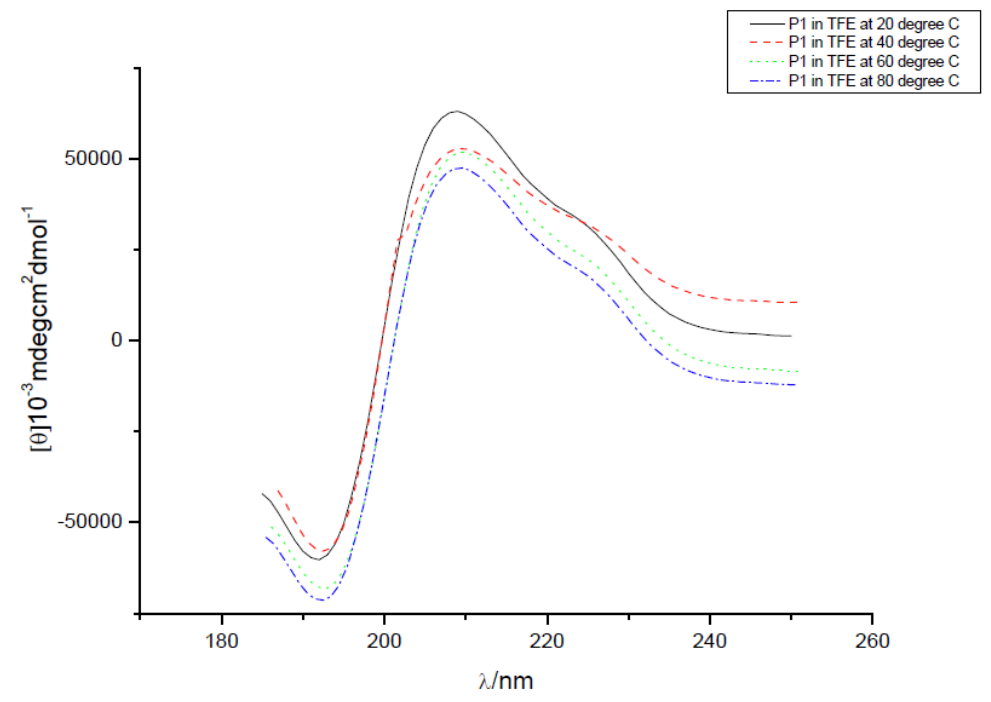

A

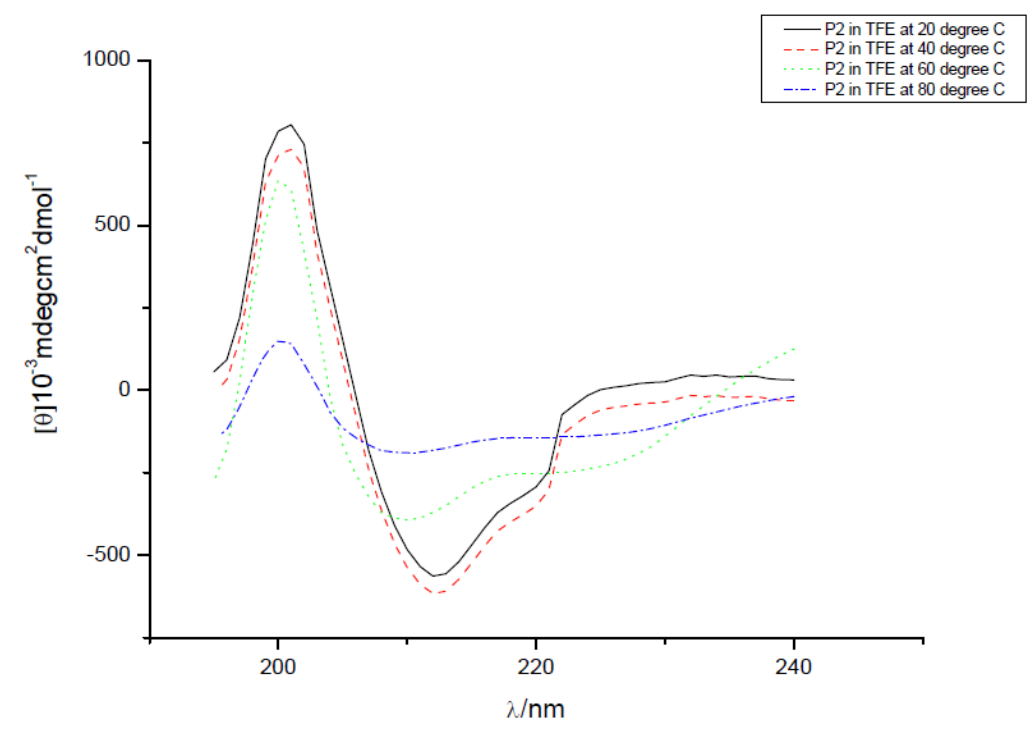

B

Figure 5.11: A comparison CD-spectroscopic profile of a 14-helical peptide, P1 (A) and a 10/12helical peptide, $\boldsymbol{P 2}(\boldsymbol{B})$, at different temperatures in TFE to determine the peptide stability

It is evident from the CD-profile (A), that although the helical content (ellipticity) of the 14helical transmembrane peptide with a definitive helical macrodipole moment, P1, got slightly reduced with the increase in temperature from $20^{\circ} \mathrm{C}$ to $80^{\circ} \mathrm{C}$, but the 14-helical peptide was still quite stable at temperature as high as $80^{\circ} \mathrm{C}$ and retained its 14-helical conformation in TFE.

On the other hand, the CD-profile (B) clearly depicted a major change in pattern. The helical content (ellipticity) of the 10/12-helical transmembrane peptide with zero-macrodipole 
moment, P2, was gradually decreased with the increase of temperature from $20^{\circ} \mathrm{C}$ to $60^{\circ} \mathrm{C}$, but from $60^{\circ} \mathrm{C}$ to $80^{\circ} \mathrm{C}$, the helical content dropped drastically, indicating a major destabilization in the helical secondary structure in TFE.

So, comparing the two outcomes from the temperature dependent CD-spectra between the 14helical and 10/12-helical peptides, it could be concluded that the major difference between the two secondary helical structures being the dipole moment, it must have played a crucial role in stabilization of the helical secondary strctures. In case of the 14-helical peptide, P1, the inverted helical macrodipole moment (with respect to the $\alpha$-peptides) held the secondary structure energetically more rigid and so even the higher temperature as $80^{\circ} \mathrm{C}$, could not destabilize the helical structure and the peptide retained its original helicity in TFE. Nevertheless, in case of the 10/12-helical peptide, P2, had almost no macrodipole momnet present due to the nullification of the latter beacuse of the alternate parallel and perpendicular orientations of the individual amide dipole moments. So, the 10/12-helicla secondary structure was less energetically rigid and it required comparativly less energy to destabilize the conformation. Therefore, between $60^{\circ}-80^{\circ} \mathrm{C}$ temperatures, the $10 / 12$-helical structure was considerably destabilized, which was evident from the drastic reduction in helicla content (ellipticity) in this temperature range.

Hence, it could be finally concluded that the helical macrodipole moment did have a fundamental role in stabilizing the helical secondary structure in solution (TFE). The more the helical macrodipole moment of the peptide, the more is it stable against temperature in slution (TFE).

\subsubsection{Secondary structure of target 6 -peptides inside lipid vesicles}

As the insertion of the target transmembrane peptides into specific lipid vesicles at definite conditions has already been confirmed by the Trp-fluorescence spectroscopy, the next task was to investigate whether the inserted $\beta$-peptides maintained their characteristic helical secondary structures inside the lipidic environment. In analogy to the CD-spectroscopic investigations performed for the peptides into solution (TFE), the same has been carried out to shed light of the secondary structures of those peptides when inside the lipid vesicle.

The peptides, P1 (14-helical D- $\beta^{3}$-Val/Ala/Leu triad peptide), P10 (14-helical D- $\beta^{3}$-Val-peptide), P11 (12-helical D- $\beta^{3}$-Leu-peptide), P2 (10/12-helical D- $\beta^{3} / \beta^{2}$-Val/Ala/Leu-peptide) and P3 (10/12-helical D- $\beta^{2} / \beta^{3}$-Val/Ala/Leu-peptide), were inserted in three different lipid vesicles, DLPC, DMPC and POPC with peptide to lipid (P:L) ratio kept constant at 1:50 along with the peptide concentration at $30 \mu \mathrm{M}$. The choice of the lipid vesicles and the peptides were based on the extent of negative hydrophobic mismatch betwwen the peptide and the lipids. The lengths of 
all these peptides were comparable while the hydrophobic bilayer thickness of DLPC almost perfectly matched with the hydrophobic core lengths of this peptide and the negative mismatch increased gradually from DMPC to POPC to an extent of $6 \AA$ to $10 \AA$, respectively. Specific percentages of $\mathrm{EtOH}$ was used to compensate the negative hydrophobic mismatch by reducing the lipid bilayer thickness. After the insertion of the peptides into these lipid vesicles at different conditions were confirmed by Trp-fluorescence spectroscopy, the CD-spectroscopic investigations were carried out to check on whether the inserted transmembrane peptides also retained their original secondary helical structures inside lipid environment.

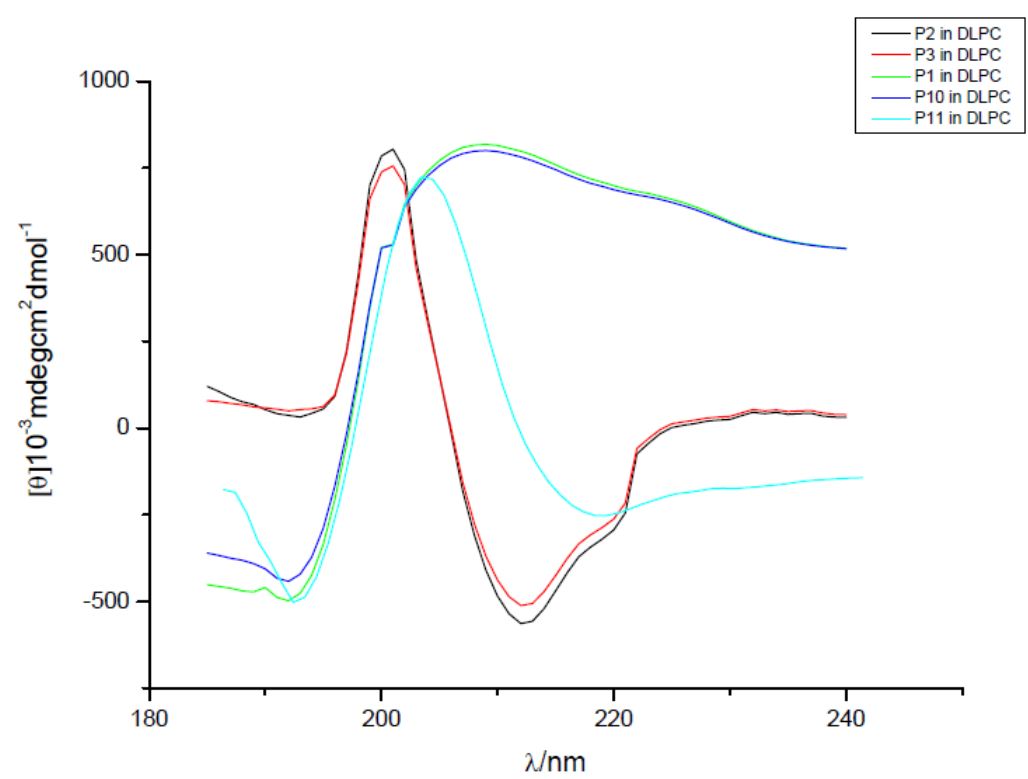

A

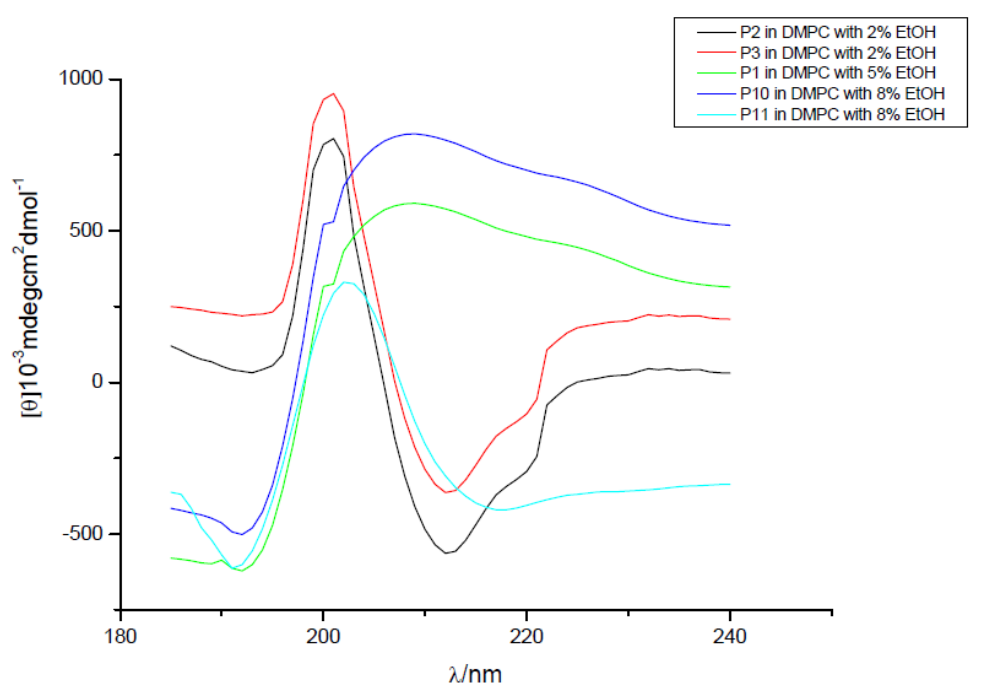




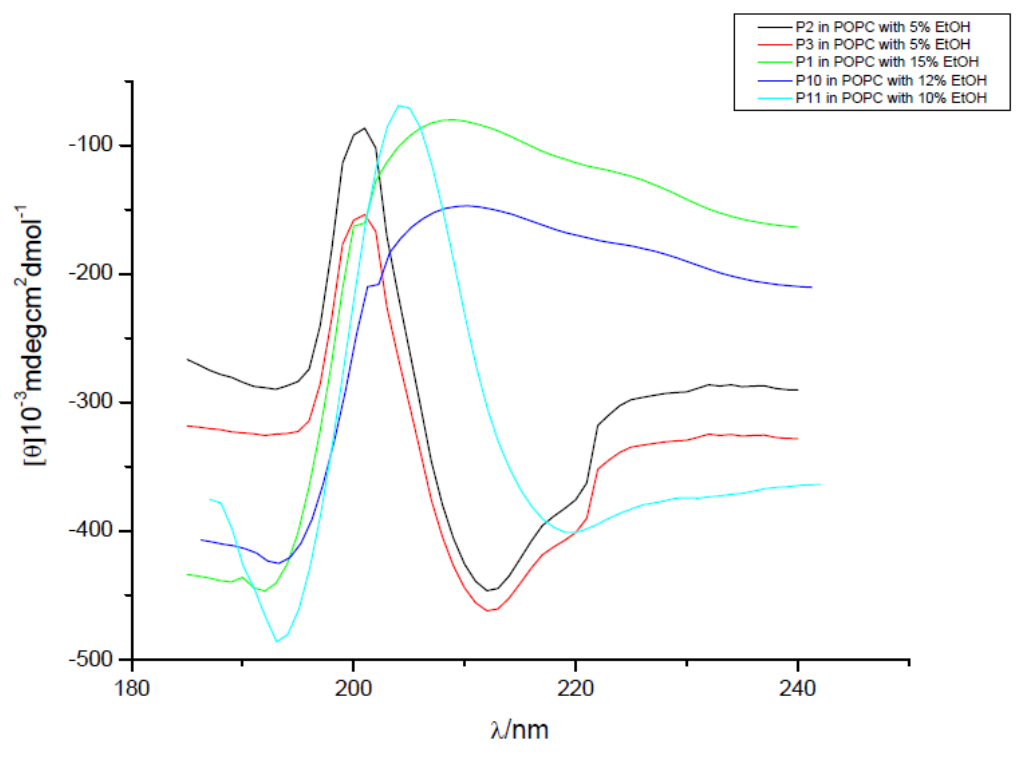

C

Figure 5.12: $C D$-spectroscopic profiles of peptides $\boldsymbol{P 1}, \boldsymbol{P} 2, \boldsymbol{P} 3, \boldsymbol{P} 10$ and $\boldsymbol{P} 11$ in $D L P C(\boldsymbol{A}), D M P C(\boldsymbol{B})$ and POPC (C) large unilamellar vesicles with $P: L=1: 50$, temperature $50^{\circ} \mathrm{C}$ and Concentration(peptide) $=30 \mu \mathrm{M}$

\begin{tabular}{|c|c|c|c|c|c|}
\hline DLPC & P1 & P2 & P3 & P10 & P11 \\
\hline Maxima & $208 \mathrm{~nm}$ & $201 \mathrm{~nm}$ & $202 \mathrm{~nm}$ & $209 \mathrm{~nm}$ & $206 \mathrm{~nm}$ \\
\hline Minima & $193 \mathrm{~nm}$ & $212 \mathrm{~nm}$ & $213 \mathrm{~nm}$ & $192 \mathrm{~nm}$ & $193 \& 218 \mathrm{~nm}$ \\
\hline
\end{tabular}

\begin{tabular}{|c|c|c|c|c|c|}
\hline DMPC & P1 & P2 & P3 & P10 & P11 \\
\hline Maxima & $207 \mathrm{~nm}$ & $202 \mathrm{~nm}$ & $201.5 \mathrm{~nm}$ & $208 \mathrm{~nm}$ & $207 \mathrm{~nm}$ \\
\hline Minima & $192 \mathrm{~nm}$ & $210 \mathrm{~nm}$ & $212 \mathrm{~nm}$ & $191 \mathrm{~nm}$ & $194 \& 219 \mathrm{~nm}$ \\
\hline
\end{tabular}

\begin{tabular}{|c|c|c|c|c|c|}
\hline POPC & P1 & P2 & P3 & P10 & P11 \\
\hline Maxima & $205 \mathrm{~nm}$ & $201.5 \mathrm{~nm}$ & $201 \mathrm{~nm}$ & $206 \mathrm{~nm}$ & $205 \mathrm{~nm}$ \\
\hline Minima & $191 \mathrm{~nm}$ & $211 \mathrm{~nm}$ & $213 \mathrm{~nm}$ & $193 \mathrm{~nm}$ & $193 \& 217 \mathrm{~nm}$ \\
\hline
\end{tabular}

Table 5.3: CD-spectroscopy results of peptides, P1, P2, P3, P10 and P11 in three different - lipids, DLPC, DMPC and POPC at-a-glance

From the results depicted in Table 5.3, it was evident that all the peptides retained their original secondary helical structures inside three different lipids. So, it was confirmed that all the three 
three types of synthesized $\beta$-peptides with secondary helical structures, 14-helix, 12-helix and alternate 10/12-helix, retained their native secondary helical structures inside lipid bilayer systems. These experiments also showed that when the peptides were inserted inside the lipids with the use of EtOH to compensate the negative hydrophobic mismatch, that did not affect the secondary structures and the native helicities were maintained compared to that in solution (TFE).

Similarly the longer thansmembrane peptides P4 (14-helical $\beta^{3}$-Val peptide) and P5 (12-helical $\beta^{3}$-Leu peptide), were also tested in three diferent lipid systems, POPC, 22:1(Cis)PC and 24:1(Cis)PC due to the same strategy as described for the peptides P1, P2, P3, P10 and P11 previously. The two peptides were almost of cmparable hydrophobic core length and the bilayer thickness of POPC almost match with the transmembrane core lenghths of P4 and P5. But the negative hydrophobic mismatch gradually increased between P4, P5 and 22:1(Cis) PC and 24:1(Cis) PC from $6 \AA$ to 8-9 $\AA$, respectively. Different percentages of EtOH was used to compensate the negative hydrophobic mismatches by reducing the bilayer thickness of the lipids in order to insert the transmembrane peptides into the lipid membranes.

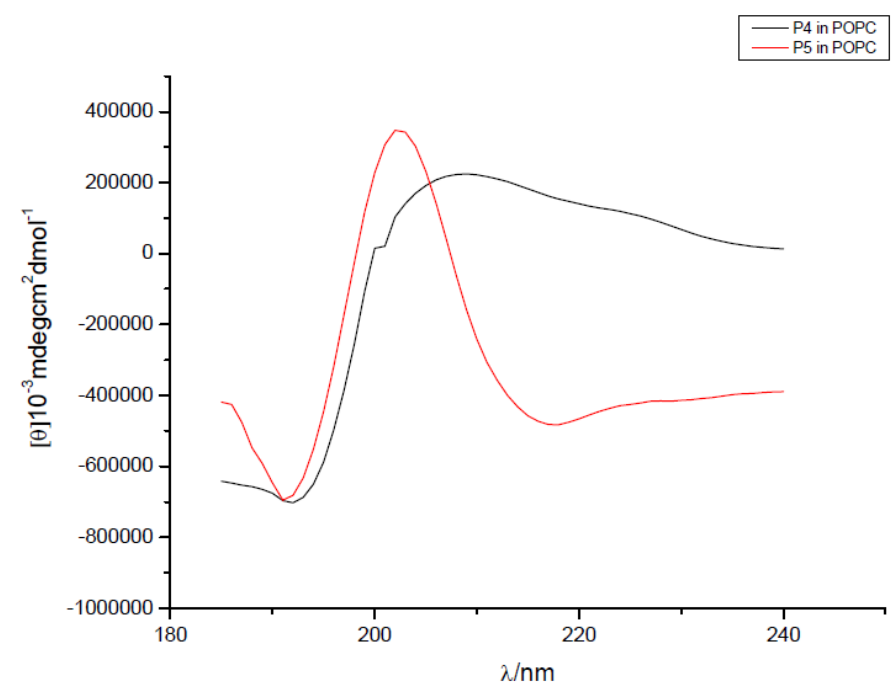




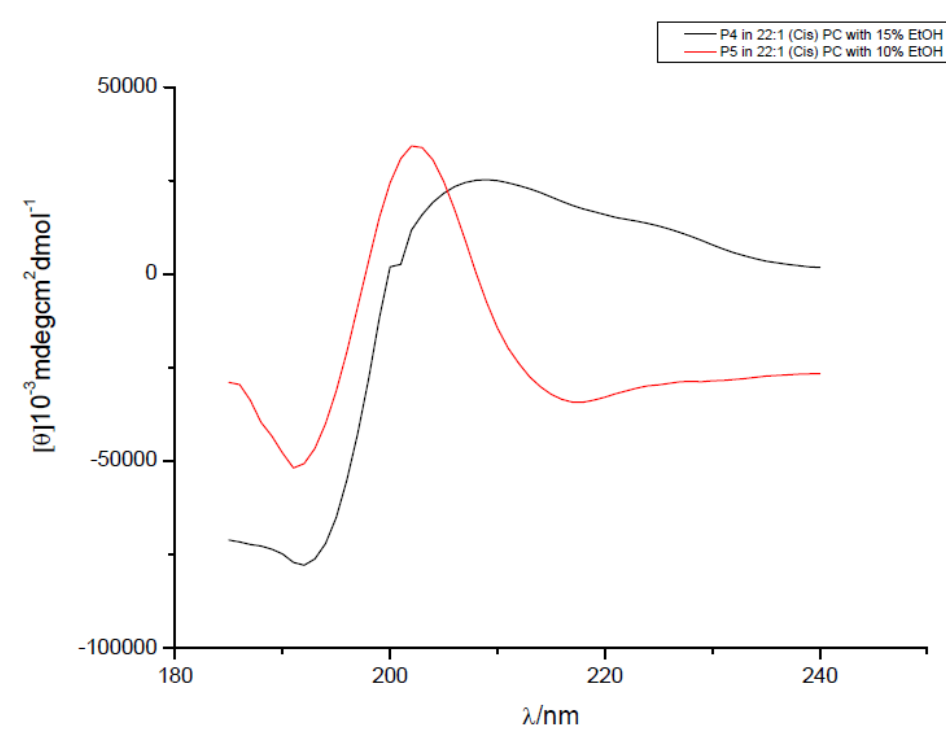

B

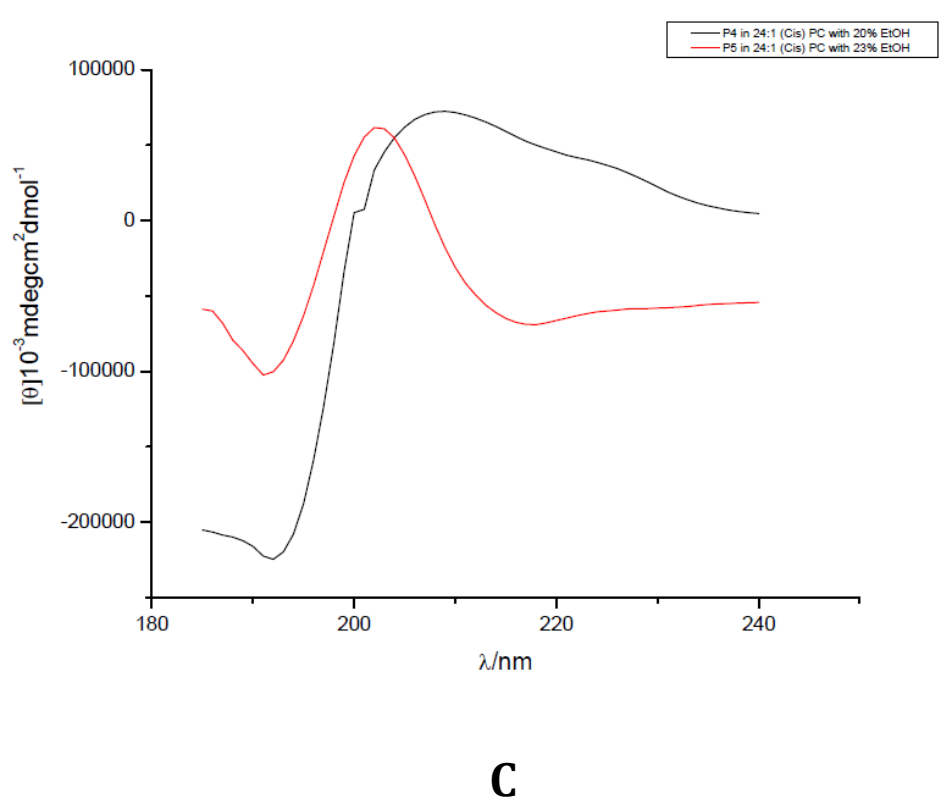

Figure 5.13: CD-spectroscopic profiles of peptides P4 and P5 in POPC (A), 22:1(Cis)PC (B) and 24:1(Cis)PC (C) large unilamellar vesicles with constant P:L ratio and peptide concentration at 1:50 and $30 \mu M$, respectivel 


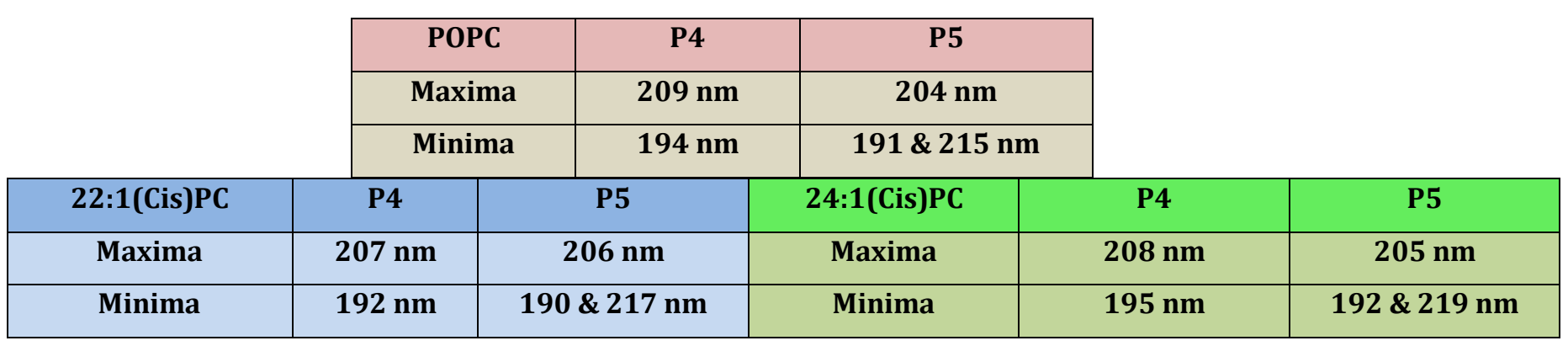

Table 5.4: CD-spectroscopy results of peptides, $\mathbf{P 4}$ and $\mathbf{P 5}$ in three different - lipids, POPC, 22:1(Cis) PC and 24:1(Cis) PC at-a-glance

From the CD-spectroscopy profiles showed in Figure 5.13 and the results depicted in Table 5.4, it was confirmed that the longer transmembrane peptides, P4 and P5, retaind their native secondary helical structures inside three different lipid systems, POPC, 22:1(Cis)PC and 24:1(Cis)PC. The results also evidently exhibited that even in case of nagative hydrophobic mismatch, when the peptides were inserted by compensating the mismatch by using EtOH, the native secondary helical structures inside the lipid environment remained the same.

Therefore, to conclude, the native secondary helical structures of the three different types of $\beta$ peptides were retained and it was further confirmed by using different lipid systems that resembled from perfect hydrophobic matched sstems to gradual negative hydrophobic mismatch scenario. But in all cases, the secondary helical structures were retained similar to that in solution (TFE).

After the confirmation of the retention of secondary elical structures inside lipid environments, it was further studied whether the stability of the 14-helical and 10/12-helical transmembrane peptides of comparable lengths in a smae lipid differed at different temperatures. In analogy to the stability studies carried out in TFE, the transmembrae peptides, P1 and P2 were selected for the similar stability studies at different temperatures starting from $20^{\circ} \mathrm{C}$ to $80^{\circ} \mathrm{C}$ inside DLPC lipid vesicle. DLPC lipid vesicle was chosen as the length of hydrophobic cores of the peptides, P1 P2, almost perfectly matched with the bilayer thickness of the DLPC large unilamellar vesicle. 


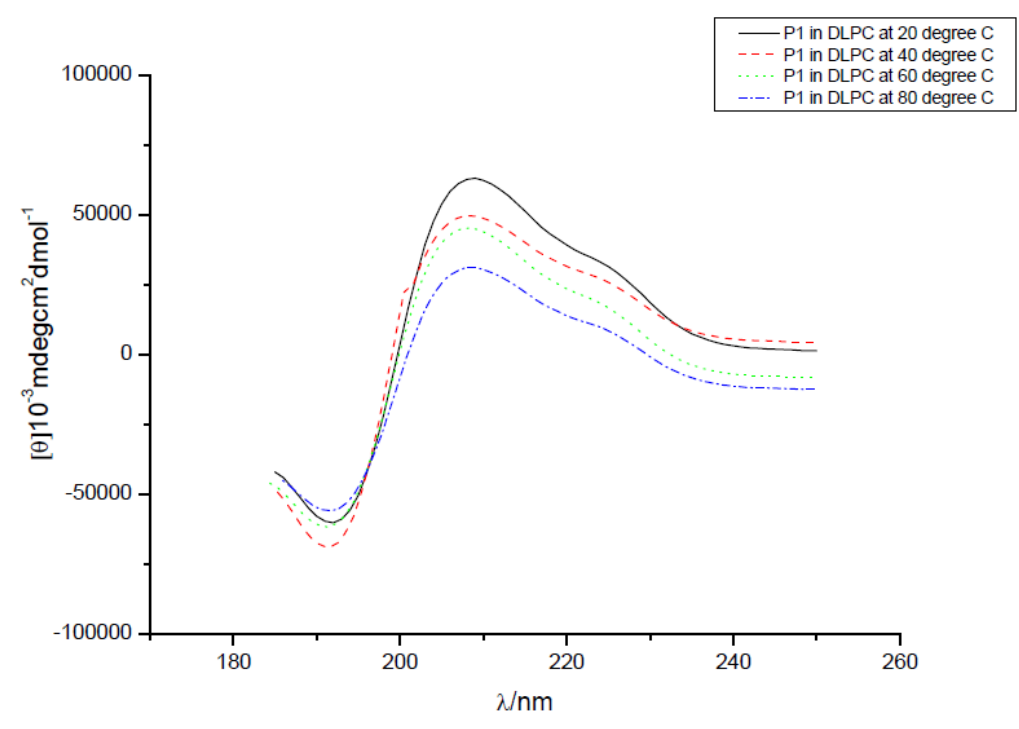

A

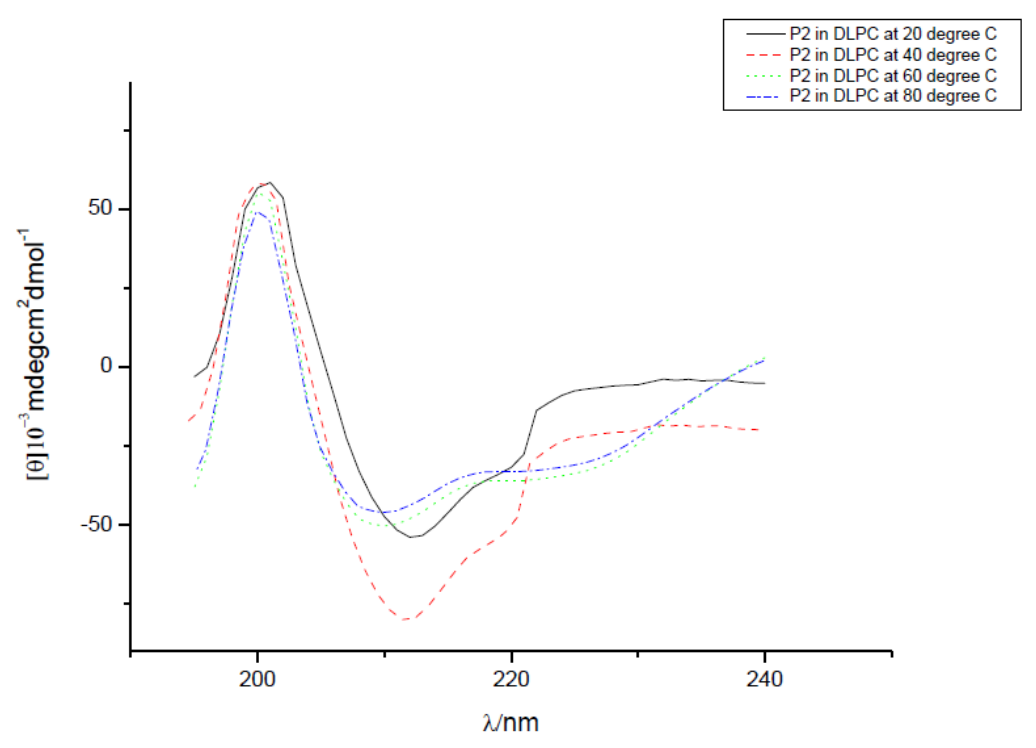

B

Figure 5.14: A comparison CD-spectroscopic profile of a 14-helical peptide, P1 (A) and a 10/12helical peptide, P2 (B), at different temperatures in DLPC to determine the peptide stability keeping $P: L$ ration and peptide concentration constant at 1:50 and $30 \mu M$, respectively

It was evident from the Figure 5.14 that even at temperaure as high as $80^{\circ} \mathrm{C}$, the peptides $\mathbf{P 1}$ and $\mathbf{P} 2$ retained their native 14- and 10/12-helical secondary helical structures, respectively. Although the helical contents were reduced gradualy with increasing temperature, but the peptides were stable enough to show their native secondary helical structures even at $80^{\circ} \mathrm{C}$. Nevertheless, there was a striking difference in stability when the 10/12-helical peptide, P2, was measured by CD-spectroscopy in solution (TFE) and inside hydrophobic lipid environment (DLPC). The difference was clearly visible by comparing Figure-5.14 (A) and Figure-5.14 (B). 
When in solution environment (TFE), the 10/12-helical peptide drastically lost its helical content and stability in the range of $60^{\circ} \mathrm{C}-80^{\circ} \mathrm{C}$. But this destabilizato was missing inside DLPC hydrophobic lipid environment. The 10/12-helical peptide was as stable as the 14-helical peptide when inside the hydrophobic lipid environment (DLPC) at hight temperature $\left(80^{\circ} \mathrm{C}\right)$. Therefore, it could be assumed that the lack of helical dipole moment made the 10/12-helical peptide more hydrophobic in nature than that of the 14-helical peptide. So, the hydrophilic environment in solution (TFE) stabilized the 14-helix more than the 10/12-helix and due to this extra stabilization of 14-helix in solution, the stability of 14-helix was more at higher temperature. On the other hand, the environment inside lipid bilayer was hydrophobic which stabilized the more hydrophobic zero-dipole 10/12-helical peptide, P2, more than that of the 14helical peptide, P1. Due to this extra stabilization of the 10/12-helical zero-dipole peptide inside lipid bilayer, it was equally stable as the 14-helical peptide even when lacking the helical dipole moment at high temperatures $\left(80^{\circ} \mathrm{C}\right)$. Therefore, it was concluded that inside lipid bilayer, the lack of helical dipole moment played a key role in the increased stability of the 10/12-helical peptides

against high

temperatures.

\section{6. $\beta$-Peptides: An Outlook towards Cell Penetration and Possible Effect of Macro- Dipole Moment}

\subsection{General}

The biological membrane regulates the permeation of substances across and acts as an intelligent limiting barrier between the intra- and extra cellular environments. This regulation is very important for individual cells to function properly and surviving. There are several therapeutic agents developed by the scientific community that are not permitted by the biological membrane to enter into the cells. $[167,168]$ So, the therapeutic application of these drugs are predominantly dependent on the development of effective vector agents that are able to get attached to the target drug molecules and deliver them into the cells through the biological 
membrane. There have been numerous approaches to carry out this function by electroporation, microinjection, liposomal formulation and use of viral vectors. [169, 170] But none of the approaches proved to be efficient due to poor cell-specificity and arising side-effects due to cytotoxicity. ${ }^{[171,172]}$ So, a class of short-chain (less than 30-amino acid long) peptides evolved as the potential candidates to carry the membrane impermeable therapeutic agents into the cell. These intra-cellular drug delivery vehicles were known as the `cell penetrating peptides' (C.P.P). The cell penetrating peptides are widely used due to their unique capability to deliver a cargo across the membrane both in vitro and in vivo, where the cargo itself is impermeable through the membrane. The cell penetrating peptides became extremely popular and of great scientific interest since the discovery of its first type, TAT derived from human immunodefficiency virus (HIV), in 1988. [173] The CPPs were primiarily short-chain peptides composed mostly of cationic amino acid residues, like, Arg, Lys and His, that particularly had the capability to traslocate through the cell membrane via different mechanistic routes, including direct penetration by membrane pore formation, to deliver a variety of cell-impermeable therapeutic cargos across the biological membrane[174], including proteins[175], nucleic acids[176], peptide-nucleic acids[177], small molecule therapeutics[178], siRNA[179], quantum dots[180] and MRI-contrasting agents[181]. The extensive research on developing numerous types of cell penetrating peptides was pursued during last three decades mainly due to their versitality coupled with easy functionalization of the linked target cargo molecules along with the high delivery efficiency into various cell lines, overcoming the challenges often faced with the other delivery strategies. $[182,183]$ Although there are plenty of potential CPPs available for intra-cellular cargo delivery at this moment, their lack of proteolytic stability and cationic nature gave rise to numerous cytotoxic effects in long term. ${ }^{[184]}$ The positively charged amino acid residues in the cell penetrating pepides played a key role in direct membrane penetration via pore formation but the accummulation of the ositively charged cell penetrating peptides inside liver and other organs posed a significant threat. [185, 186] On the other hand, highly hydrophobic cell penetrating peptides had issues of poor solubility and self-aggregation in aqueous environment. $[187,188]$

To address these shortcomings of most of the standard positively charged cell penetratig peptides available, the synthesized two types of $\beta$-peptide sequences, P6, P7 (10/12-helical) and P8, P9 (14-helical), were tested to investigate whether they possessed any possibble cell penetrating efficiencies. These peptides were 6-amino acid long and were composed only of a triad of hydrophobic amino acid sequences (Val/Ala/Leu). The peptides P8, P9, consisted only D- $\beta^{3}$-(Val/Ala/leu) triad whereas the P6, P7, of alternating D- $\beta^{3} / \beta^{2}(\mathrm{Val} / \mathrm{Ala} / \mathrm{Leu})$ triad. These peptides had no hydrophilic or any charged residues, so the threat of cytotoxicity and physiological damage by accummulation of the charged peptide residues in liver and other 
organs could be omitted. On the other hand, $\beta$-peptides in general were reported to be more stable than that of the $\alpha$-peptide analogue and were especially stable under proteolytic enzymes. Hence, the two major shortcomings of most of the avilable cell penetrating peptides could efficiently be addressed by investigating any possible efficiency of the two different types of $\beta$ peptides in cell penetration.

\subsection{Cellular Uptake Mechanisms: A Brief Overview}

Despite extensive studies made on different classes of cell penetrating peptides, the actual mechanism by which the CPPs penetrate and enter cells could not yet be completely resolved. One reason behind such complexity was that the process of cellular uptake did not follow a single mechanistic route and often found to occur as a combination of different mechanistic routes. Besides, the mechanistic route greatly daviated depending on the changes in the parameters, such as, the peptide concentration, peptide to lipid ratio, temperature, cell lines, incubation time, etc. $[189,190,191]$ Hence, it was indeed difficult to make a general conclusion on the mechanism of cellular uptake even for a single CPP because all the possible mechanistic routes were somehow connected to each other some of them also occured simultaneously. Therefore, a brief idea on some of the major possible cellular uptake mechanistic routes involving direct translocation strategy that were thought to be significant for the cell penetration studies of the synthesized $\beta$-peptides and the reference CPP, HIV-1 TAT, have been discussed in the following section.

\subsubsection{Inverted Micelle Formation}

This mechanistic route was first proposed to explain the direct penetration of the peptides by translocation. [192] The model suggested that the positively charged residues of the cell penetrating peptide would interact with the negatively charged phospholipids in the biological membrane and the hydrophobic residues subsequently interacted with the apolar core of the lipid membrane. This in turn induced a destabilization in the membrane system giving rise to a negative curvature. This phenomenon was also known as 'invagination of the membrane'. [193] The reorganization of the neighbouring lipid molecules took place due to the invagination and this led to the formation of inverted micelle structures that encapsulated the peptides in their interior. [194] The membrane destabilization and formation of inverted micelle structures ultimately released the encapsulated peptides at the intracellular side. Thus the peptides got 
penetrated through the lipid membrane by forming inverted micelle structures (Figure 6.1A).[195, 196]

\subsubsection{Adaptive Translocation}

The cell membrane consisted of an array of negative charges, so the significant role of cationinc amino acid residues in CPP design were thoroughly exploited. Long time ago, Ryser and Hancock found out that the cellular uptake of albumin was drastically increased if the protein was mixed with high molecular weight poly-lysines. [197] The effect of the positively charged amino acid residues in CPP to penetrate cell membranes could logically be explained by the attachment of the cationic amino acid residues to the membrane by the electrostatic interactions between the basic amino acid residues and the negatively charged polysachharides and lipids in membrane followed by the internalization of the CPPs into the intra-cellular media. This explanation was furthr supported by several other experiments, such as, substitution of any positively charged amino acid residues by the hydrophobic neutral alanine residue in TAT-peptides significantly reduced the rate of cellular uptake.[198] It was further observed that not only the positive charge was important, but the functional group also had crucial effects in cellular uptake. Lysine and Arginine both being basic in nature, replacement of lysine residues with the arginine readily increased the rate of uptake.[198, 199] So, the increased uptake efficiency in this case could be attributed to the guanidium headgroup of the arginine side chain rather than the positive charge alone. [200, 201] The guanidium group could form bidentate hydrogen bonds with the negatively charged phosphate, carboxylate and sulfate groups on the cell surface, whereas the ammonium cations in lysines could only form monodentate hydrogen bonds. This gave rise to counteranion scavenging that supported in attenuating the poarity of the guanidium functional group by generating an ion-pair complex capable of diffusing into the membrane. ${ }^{[201]}$ The ability of the guanidium headgroups to form bidentate hydrogen bonds gave the oligoarginines either a hydrophilic or a hydrophobic character depending on the associated counteranions. By virtue of this capability, the interaction between the peptides containing multiple guanidium side chains and the phosphate lipid head groups would mask the peptide charge, attenuating its polarity and allow it to adaptively diffue through the membrane. [202] The diffusion was always driven by the membrane potential and hence the peptide must carry a positive net charge to be able to penetrate cell membrane by adaptive translocation (Figure 6.1C). [201] 


\subsubsection{Pore Formation}

The molecular dynamic studies and electrophysiology experiments have already indicated the formation of transient pores in the membrane allowing passive diffusion of ariginine-rich and TAT-peptides across the plasma membrane (Figure 6.1B). [203, 204] The electrostatic interaction between the positively charged amino acid residues and negatively charged phosphate headgroups in lipids resulted in the accummulation of the peptides in the outer leaflet of the membrane, between the phosphate and carbon-chains of the lipids. The accummulation caused a perturbation and overall thinning of the plasma membrane. The electrostatic force of attraction between the positively charged side chains of arginine or lysines and the negatively charged phosphate groups in lipid led to the formation of transient toroidal pores in the membrane through which the peptides got actively translocated accross the plasma membrane. Further investigations on this mechanism with other type of CPPs, also suggsted that formation of transient pores were a direct translocation mechanism.[205, 206, 207, 208] Palm-Apergi et al. proposed that perturbation of the plasma membrane by the formation of transient pores triggered the membrane-repair-response (MRR) process induced by calcium ion efflux into the cells. In due course the internal vesicles got fused together to serve as a patch to reseal the membrane pores. ${ }^{[209]}$

A
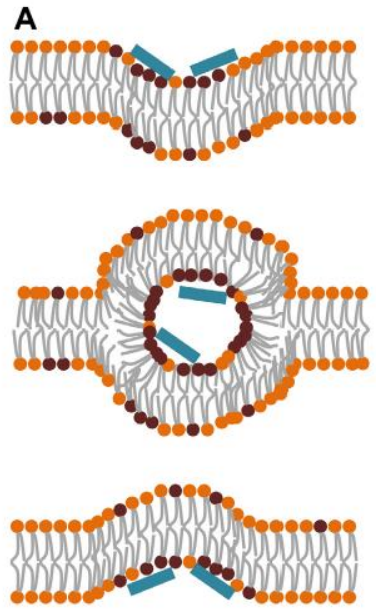

B
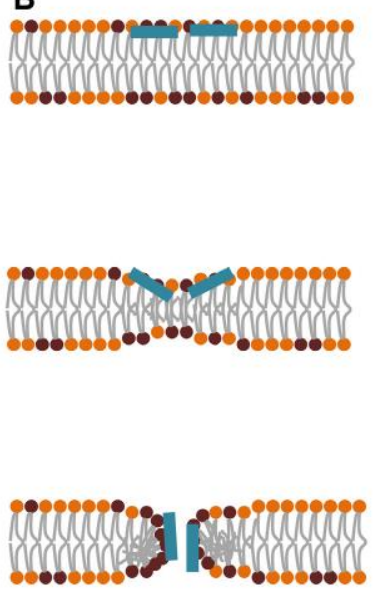
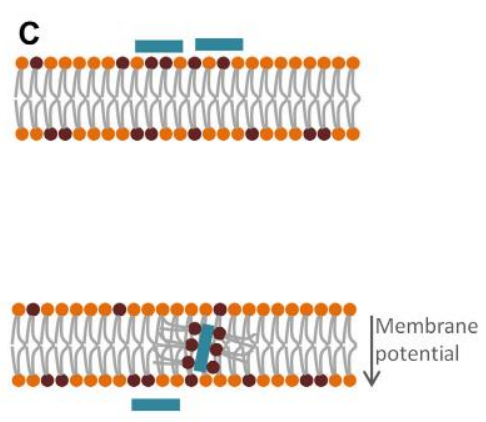

Positively charged CPP

Negatively charged phospholipid

Zwitterionic phospholipid

Figure 6.1: Schematic illustration of the possible mechanistic routes for direct membrane translocation of the cell penetrating peptides by forming inverted micelles $(\boldsymbol{A})$, forming transient pores $(\boldsymbol{B})$ and by adaptive translocation $(\boldsymbol{C})^{[210]}$ 


\subsection{Design}

It has repeatedly been indicated by the scientific reports that $\beta$-peptides were one of the most promising candidates to mimic natural $\alpha$-peptides in different biological processes with enhanced proteolytic stability, there have been very few experimental investigations carried out to test the cellular uptake efficiencies of short-chain $\beta$-peptides. Sufficient efforts have not been made to shed light on the capabilities of different types of $\beta$-peptides in cell membrane penetration. One of the main reasons behind the lack of reports in this field was the synthetic challenges to access different types of $\beta$-peptides and their propensity to get folded into different types of helical secondary structures. So, a preliminary trial was carried out to test cytotoxicity at different peptide concetrations, cellular uptake efficiency of two different types of short-chain $\beta$ peptides and also to investigate on any differential behaviours by the two types of $\beta$-peptides in connection to cellular uptake efficiency.

Although it was previously discussed in section 6.2, that presence of overall positive charged charater in the candidate peptide had crucial roles in cellular uptakes by direct translocation accross the plasma membrane, but it was also been indicated in section 6-1, that one of the major physiological side effects caused by the accummulation of the candidate peptides in different organs, like, liver, evolved due to the positively charged nature of the peptides. On the other hand, most of the cell penetrating peptides requied very high dosage as only a smaller fraction of these could ultimately deliver the cargo to the intra-cellular environment and the rest were decomposed by the proteolytic enzymes. To overcome these two major disadvantages, the idea of testing the following two types of $\beta$-peptide sequences (P6, P7 and P8, P9) as initial candidates for cellular uptake came into play. A standard and historically known cell penetrating peptide, HIV-1 TAT and its fluorescently labeled analogue, 5-FAM-HIV-1 TAT, were also selected as standards for all the measurement done with the $\beta$-peptide sequences for comparison.

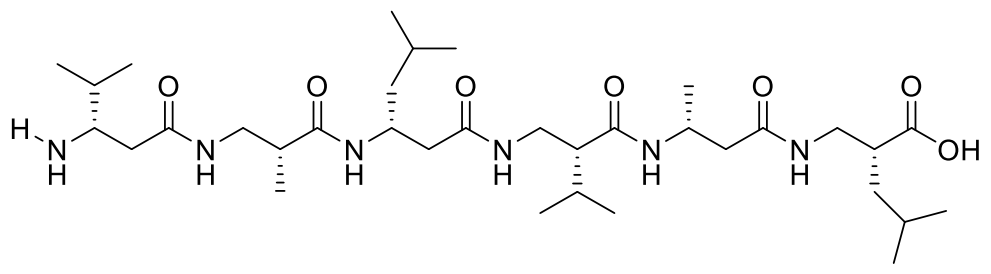




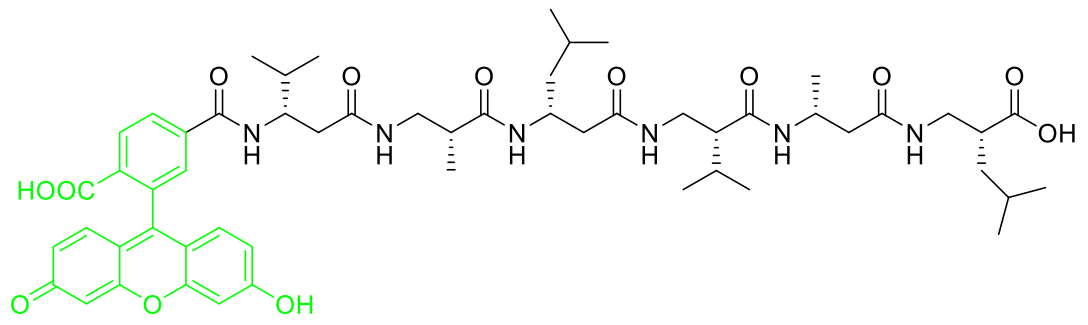

P7

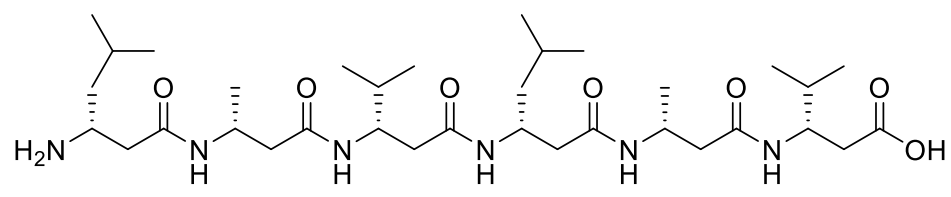

P8

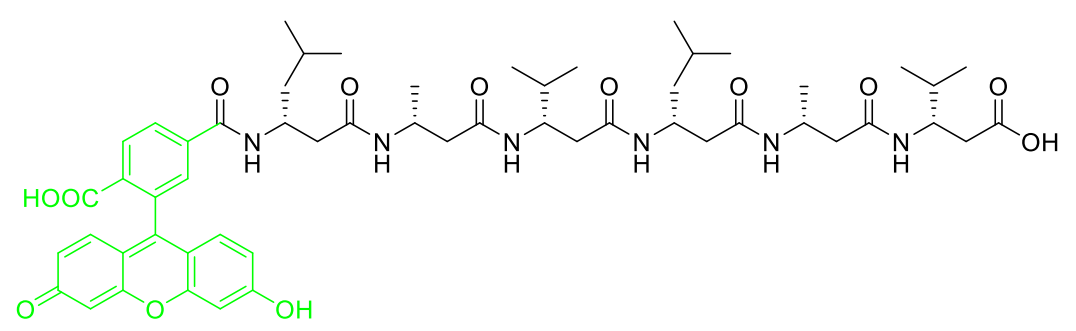

P9

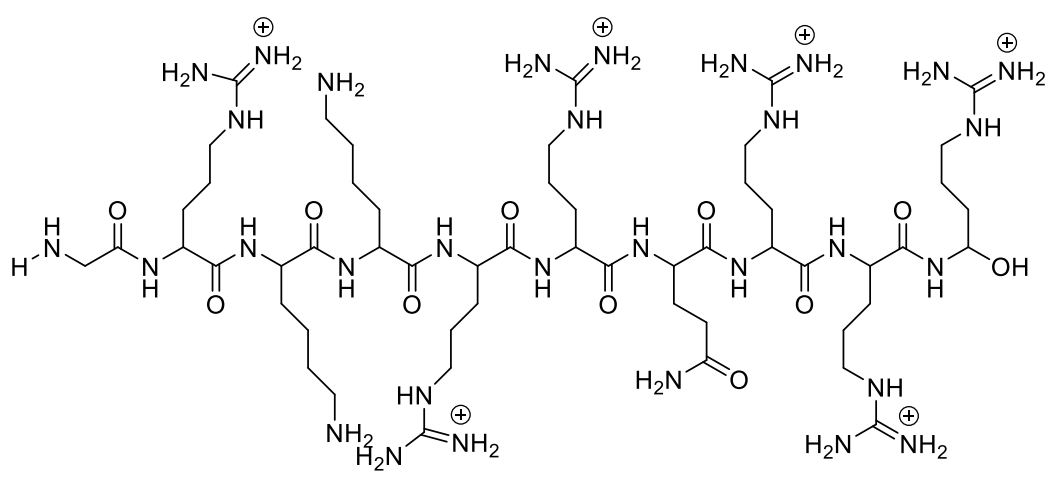

HIV-1 TAT-Peptide 


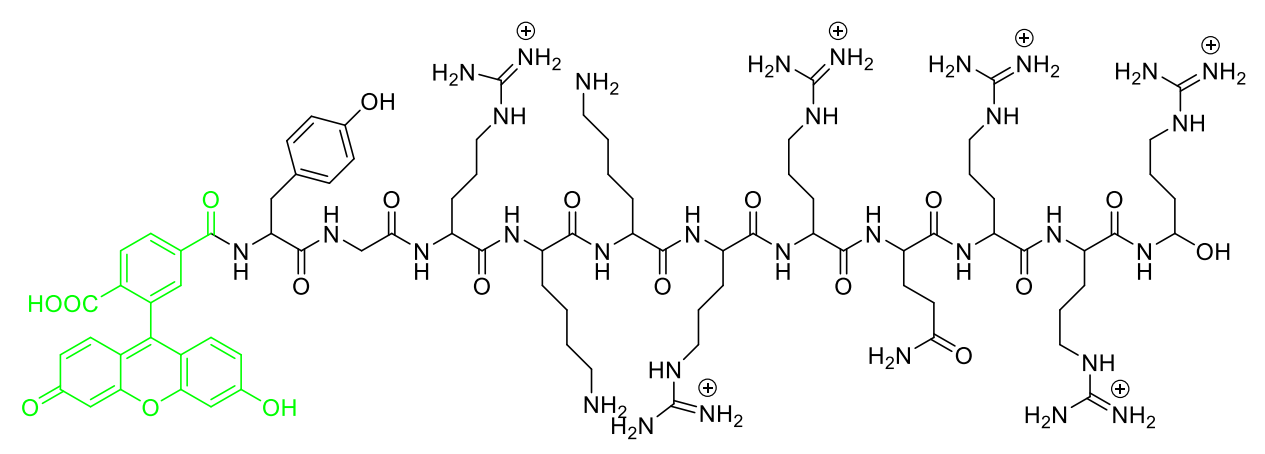

\section{5-FAM-HIV-1 TAT-Peptide}

The candidate $\beta$-peptides were designed in such a way that both of the types were devoid of any positive charges and were composed of completely hydrophobic sequences. The peptide P6 consisted an alternate $\beta^{2} / \beta^{3}$-(Val/Ala/Leu) triad sequences whereas the P8 was composed of only $\beta^{3}$-(Val/Ala/Leu) triad sequence. P7 and P9 were the 5(6)-carboxyfluorescein labeled analogues of the peptides, P6 and P8, respectively. The peptide P6 folded into a rare alternate 10/12-helical secondary structure while the P8 into a 14-helical pattern. Although both the $\beta$ peptides were devoid of any positive charges, but there were some differences between them. The peptide P8 folded into a 14-helix and had overall inverted helical macro-dipole moment in comparison to the $\alpha$-peptides. This macro-dipole moment induced a charged character into the peptides P8 and its fluorescently labeld analogue, P9. On the other hand, the peptide P6 folded into an alternating 10/12-helix where the individual dipole moments of each amides were nullified due to the alternate parallel ans perpendicular orientations of the amide dipole moments with respect to the helical axis in 12- and 10-membered hydrogen bonded rings, respectively. Therefore the overall partially charged character was completely absent in case of the 10/12-helical peptide, P6 and its fluorescently labeled analogue, P7. This made the 10/12helical peptides even more hydrophobic in nature than that of the 14-helical peptides. Although there were many other structural differences between the 10/12- and 14-helical $\beta$-peptides, but the presence and absence of overall helical macro-dipole moment was definitely one of the major ones. It was already experimentally verified that the helical macro-dipole moment could have major function in transmembrane insertion and spanning into lipid bilayers. It was observed that the more the helical macro-dipole moment, the less was the hydrophobic character of the peptide due to induction of partial charged character in it and the less was its propensity to get inserted and spanned into lipid bilayers due to unfavourable interaction between the 
hydrophobic lipid core and the less hydrophobic transmembrane segment. Now membrane insertion and spanning was a completely different procedure than that of membrane penetration and cellular uptake. So, it would be interesting to investigate whether there were any differences in cytotoxicity or cellular uptake efficiency between the two types of $\beta$-peptides and that would indicate a possible role of helical macro-dipole moment in cellular uptake. The results could further be compared to that of the membrane insertion and spanning to check whether the same trend was maintained.

\subsection{Results:}

\subsubsection{Cytotoxicity Tests by Clonogenic Assay}

Prior to proceeding with any other experiments to shed light on any possible cellular uptake efficiencies of the different types of $\beta$-peptides, it was essential to ensure whether these $\beta$ peptides had any cytotoxic effects on cells. A clonogenic assay was performed to investigate on the cytotoxicity of the two types of $\beta$-peptides in comparison to the historically known cell penetrating peptide, HIV-1 TAT. A clonogenic assay was a cell biology technique to study the possible effects of target chemical or radioactive agents on survival and proliferation of cells. This colony forming assay was long been established in 1956. ${ }^{[211]}$ Basically, the clonogenic assay enabled an assessment of the differences in reproductive viability (capacity of cells to reproduce; a single cell forming a colony of at least 50 or more cells) between untreated control cells and the treated cells with the target cehmical agents. The clonogenic survival assay was carried out in vitro using adherent human bronchial adenocarcinoma cell line A549 (ATCC CCL-185). It consisted of three distinct components;

i) Treatment of the cell monolayer in tissue culture flasks

ii) Preparation of single cell suspensions and plating an appropriate number of cells in petri dishes

iii) Fixing and staining the colonies following a relevant incubation period of 1 week. 


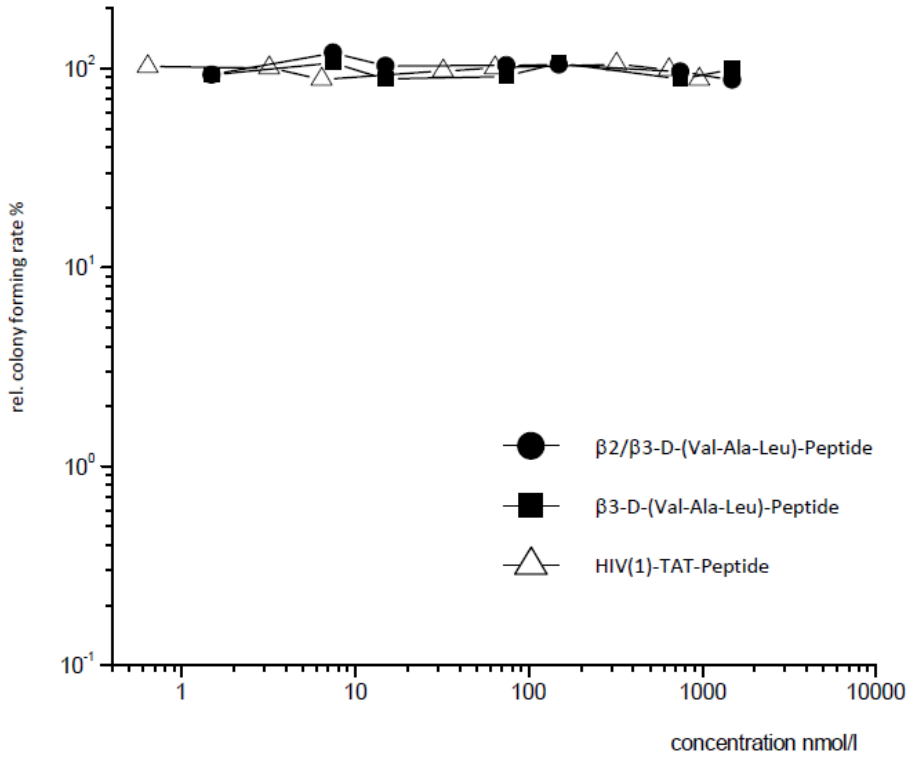

A

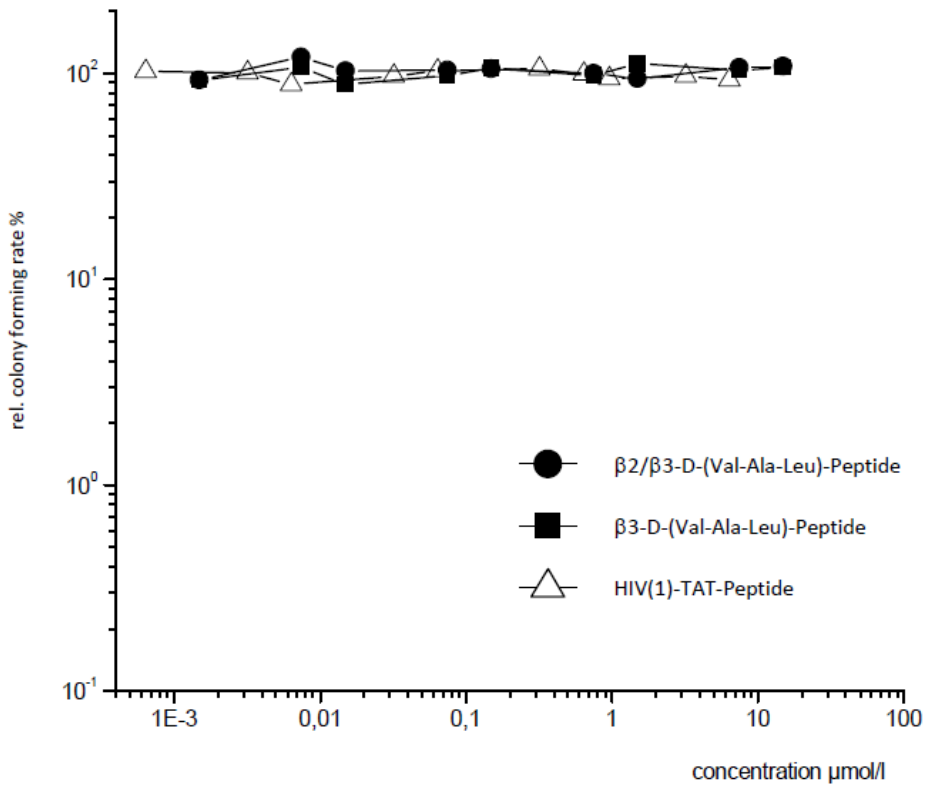

B

Figure 6.2: Cytotoxic activities of three different peptides in vitro on human adenocarcinoma cells A549 in a Clonogenic Assay; Peptide concentration upto $1 \mu \mathrm{M}$ (A) and upto $15 \mu \mathrm{M}$ (B)

\section{In the Beginning (Before Incubation)}



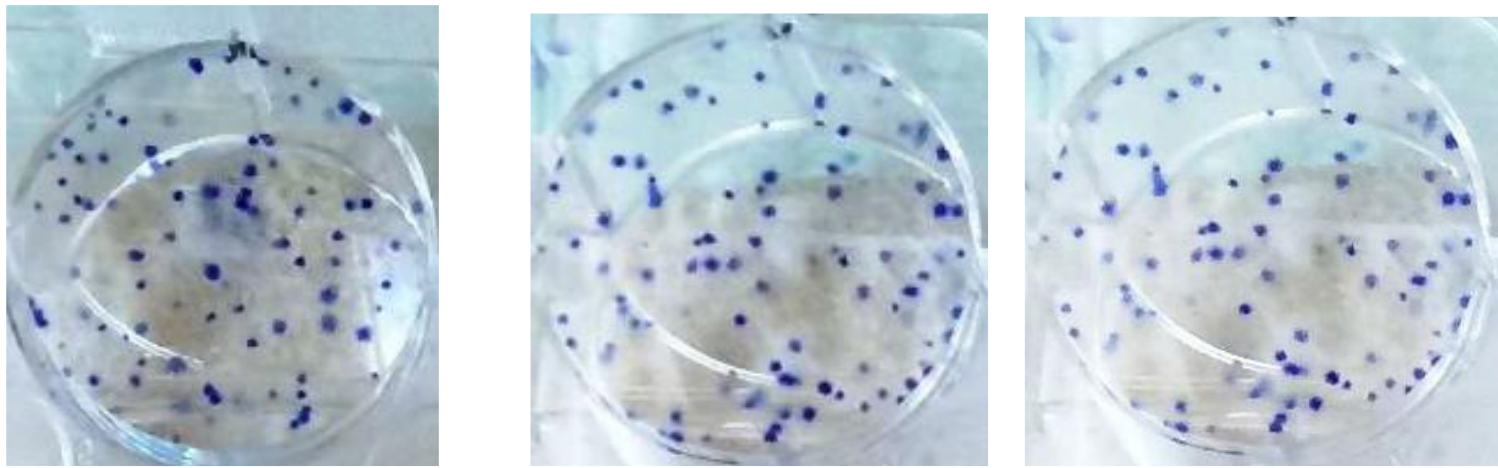

\section{After 1 Week of Incubation}

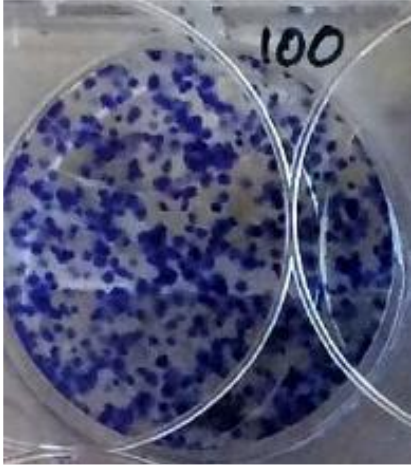

A

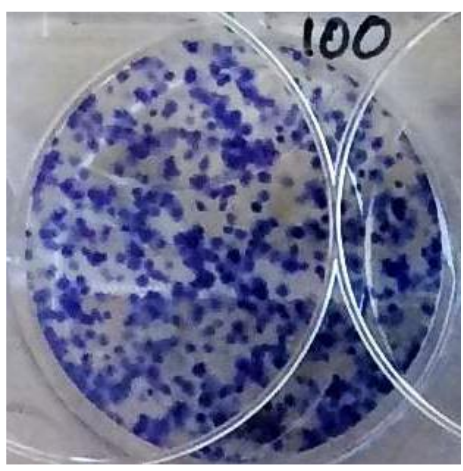

B

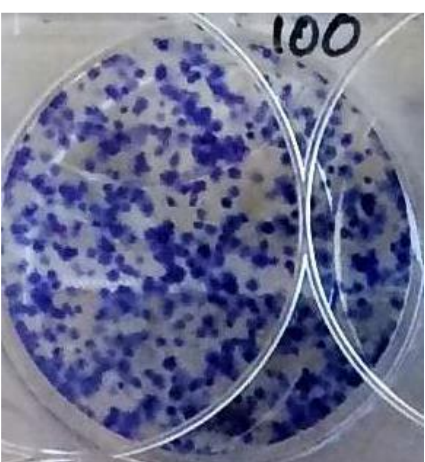

C

Figure 6.3: Digital images showing clonogenic cell colonies produced by human adenocarcinoma cells A549 following seeding of $10^{2}-10^{4}$ cells per well and one week of incubation; HIV-1 TAT Peptide (A), $\beta^{3}$-Peptide (B) and $\beta^{3} / \beta^{2}$-Peptide (C)

It was evident from the clonogenic assay that the target $\beta^{3}$-Peptide and the $\beta^{3} / \beta^{2}$-Peptide did not affect the cell proliferation efficiency of human adenocarcinoma cells A549 in comparison to the known non-cytotoxic cell penetrating peptide, HIV-1 TAT. Initially the clonogenic assay was carried out with maximum peptide concentration of $1 \mu \mathrm{M}$ (Figure 6.2 A) and when there was no cytotoxicity observed, the higher concentration was increased gradually to $15 \mu \mathrm{M}$. Still there was no cytotoxic effect observed for any of the $\beta$-peptides as well as the HIV-1 TAT peptide (Figure 6.2 B). Therfore, it could be concluded that both the $\beta$-peptides were non-cytotoxic in nature upto a high concentration of $15 \mu \mathrm{M}$. Therefore, the experiments to shed light on the quantitative cellular uptake efficiencies of the $\beta$-peptides and the HIV-1 TAT peptide were planned. 


\subsubsection{Quantitative Cellular Uptake: Fluorescence Activated Cell Sorting (FACS) Study}

In order to investigate the cellular uptake of different $\beta$-peptides and the HIV-1 TAT peptide by the fluorescence activated cell sorting (FACS) technique, fluorescently labeled analogues of all the peptides were needed. Therefore, the fluorescently labeled analogues of peptides P6 and P8, i.e, P7 and P9, respectively, were used for this experiment. FACS was basically a specialized type of flow cytometry. It provided an efficient and dependeable method to sort a heterogeneous mixture of biological cells into two or more containers, one cell at a time, based on the specific light scattering and fluorescent characteristics of each cell.

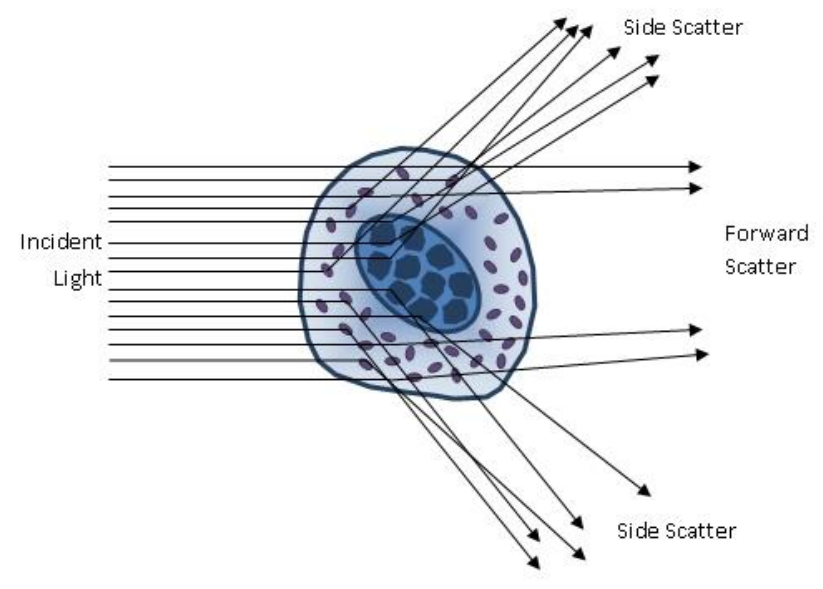

Figure 6.4: General principle of flow cytometry

In the FACS experiment, a negative control was used with untreated human adenocarcinoma A549 cells. Gating of the forward and side scatter was performed using the FACS results for the untreated negative control cells. Fluorescently labeled peptides were added to the cell line and allowed to attach for $1 \mathrm{H}$ followed by washing with the growth media and detachment from the multiplate with trypsin. Afterwards, the FACS experiments were carried out. Fluorescence was the emission of radiation following excitation by a higher energy of radiation. For example, when 5(6) carboxyfluorescein (FAM) was excited by laser of a wavelength of $490 \mathrm{~nm}$ (blue light), it emitted light at a wavelength of 519nm (green light). When the specific cells passed through the specific laser beam, they were monitored. Droplets containing single cells were given a positive or negative charge, based on whether the cell had limited the fluorescently tagged peptide or not. Droplets containing a single cell were then detected by an electric field into collection tubes according to their charge. 


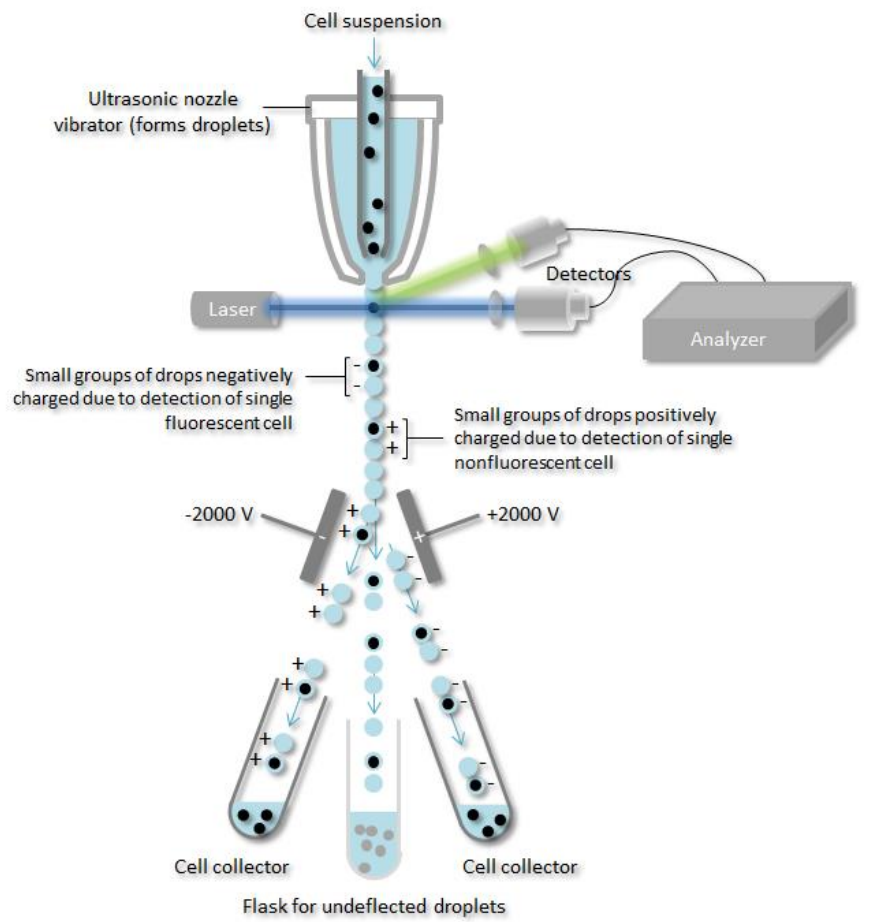

Figure 6.5: Schematic illustration of the principle of FACS

\section{Negative Control: FACS Results}

\begin{tabular}{|c|c|c|}
\hline Population & Events & \% Total \\
\hline All Events & 10370 & $\mathbf{1 0 0 . 0}$ \\
\hline Viable Events (P1) & 9694 & 93.5 \\
\hline FITC Positive & 33 & 0.3 \\
\hline Q1-1 & 243 & 2.3 \\
\hline Q2-1 & 19 & 0.2 \\
\hline Q3-1 & 9331 & 90.0 \\
\hline Q4-1 & 102 & 1.0 \\
\hline
\end{tabular}

Table 6.1: FACS Results for the Negative Control At-A-Glance 


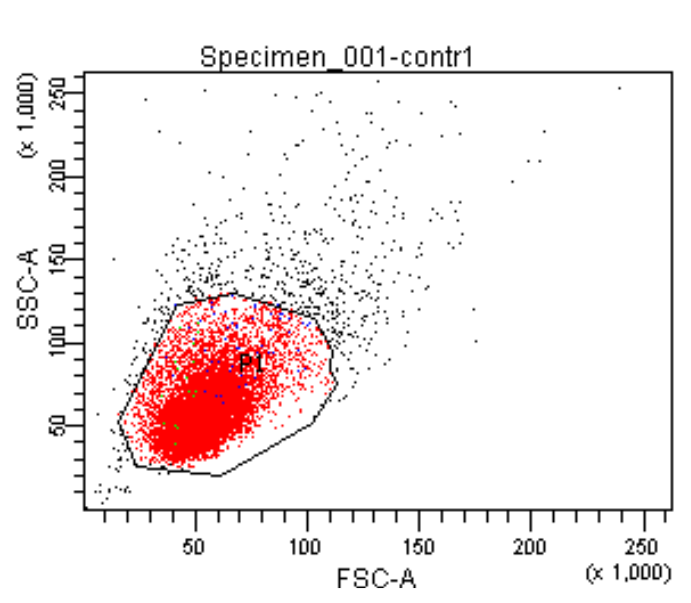

A

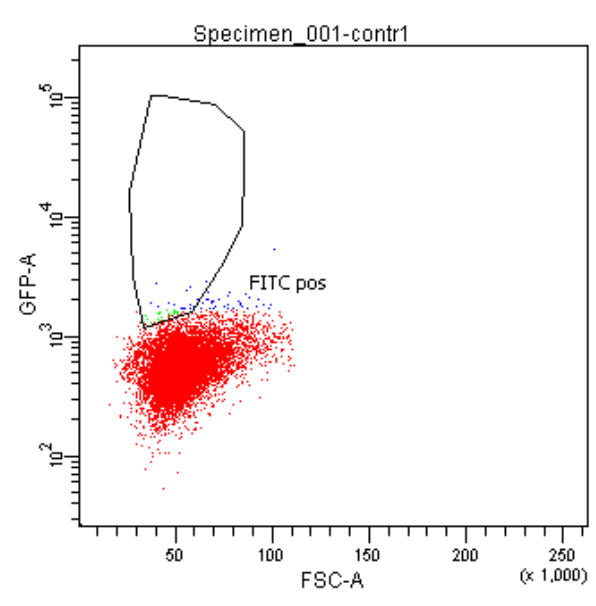

B

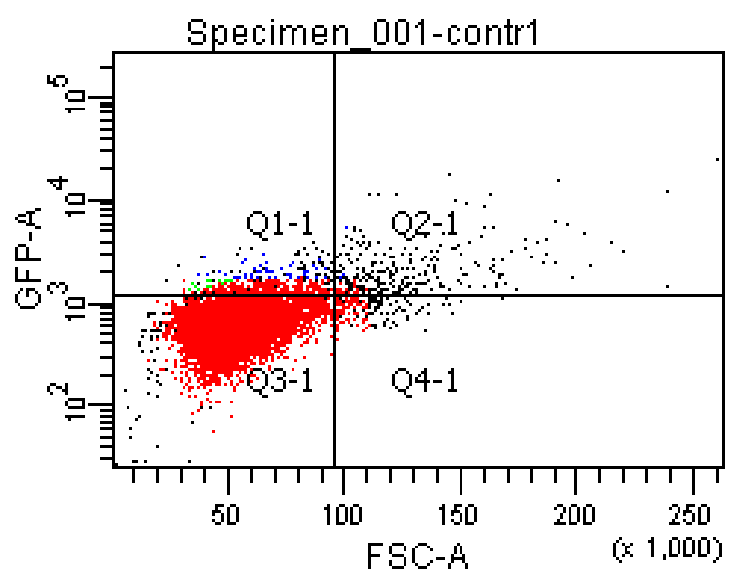

C

Figure 6.6: FACS results for untreated negative control human adenocarcinoma A549 cell lines; cell-size vs viablity (A), cell size vs. fluorescence intensity $(\boldsymbol{B})$ and quadrant divided plot of cell size vs. fluorescence intensity (C)

The Figure 6.6 and Table 6.1 cumulatively depicted the FACS results for the untreated negative control human adenocarcinoma cells A549. In Fig. 6.6A, the cell viability (SSC-A) within a limited cell sizes between 10-115 (FCS-A) were only considered, as the cell population beyond this cel size limit was perhaps not the single cells but the clusters. Therefore, a cell size boundary for the viable cells was drawn from the negative control experiment that was later used for all the other experiments. In Fig. 6.6B, the fluorescence intensity was plotted against the cell size and only the fluorescence signal was to be considered that fell into the pre-determined cell size boundary. However, as expected for the negative control cells, due to absence of any fluorescently labeled components, there was no fluorescence signal recorded inside the cell-size 
boundary. The Fig. 6.6C was only another type of illustration by a plot of fluorescence intensity against cell-size and the result was divided into four quadrants. The fluorescence signal in Q1-1 represented the single cells within the cell-size boundary with high fluorescence, Q3-1showed very low to almost nil fluorescence signal from the cells within the cell-size boundary. On the other hand, Q2-1 represented high fluorescence signal from the cells outside the cell-size boundary, whereas Q4-1 depicted very low to nil fluorescence signal from cells outside the cellsize boundary. Therefore, the population from the Q1-1 could only be considered for the positive fluorescence results, as it was the only quadrant, which showed high fluorescence signals from the single cells that fell into the pre-determined cell-size boundary. For the negative control, there was almost no population in Q1-1, as there was no fluorescently labeled species present. This could well be fitted to the results shown in Table 6.1, where the FITC positive event was negligible. Some feeble signals were recorded due to background.

\section{5-FAM-HIV-1 TAT Peptide: FACS Results}

\begin{tabular}{|c|c|c|}
\hline Population & Events & \% Total \\
\hline All Events & $\mathbf{1 0 3 8 0}$ & $\mathbf{1 0 0 . 0}$ \\
\hline Viable Events (P1) & 9607 & 93.3 \\
\hline FITC Positive & 9126 & 87.9 \\
\hline Q1-1 & 9546 & 92.0 \\
\hline Q2-1 & 118 & 1.1 \\
\hline Q3-1 & 23 & 0.2 \\
\hline Q4-1 & 0 & 0.0 \\
\hline
\end{tabular}

Table 6.2: FACS Results for the 5-FAM-HIV-1 TAT Peptide At-A-Glance 


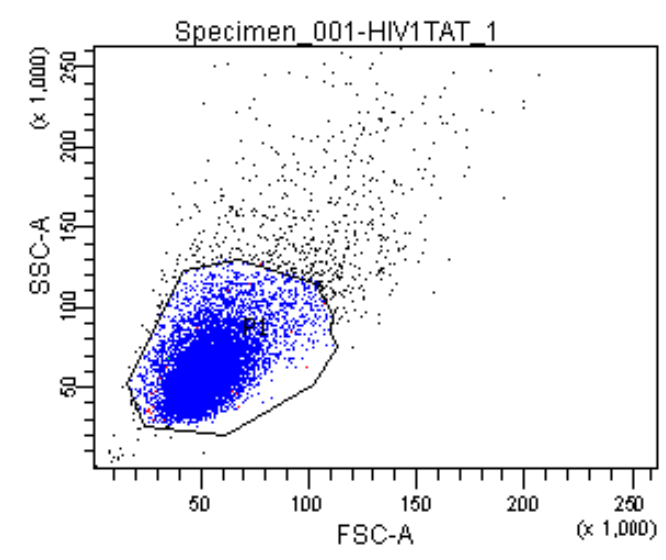

A

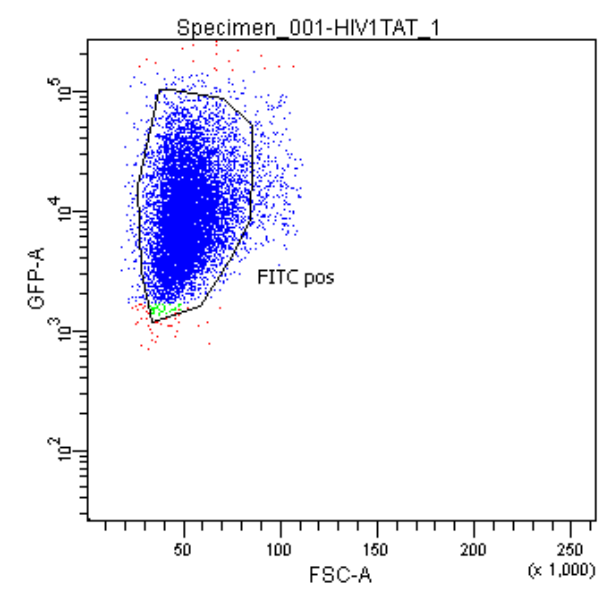

B

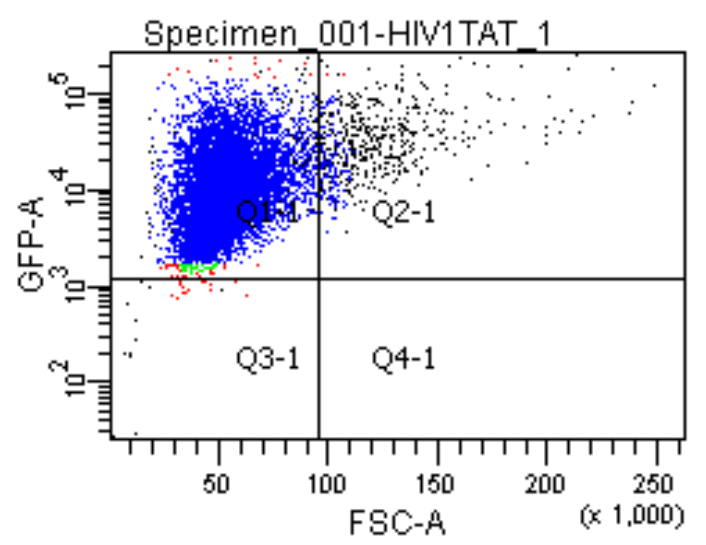

C

Figure 6.7: FACS results for HIV-1 TAT peptide treated human adenocarcinoma A549 cell line; cellsize vs viablity $(\boldsymbol{A})$, cell size vs. fluorescence intensity $(\boldsymbol{B})$ and quadrant divided plot of cell size vs. fluorescence intensity $(\mathrm{C})$

Similar to the results for negative control, the FACS results for cells treated with HIV-1 TAT peptide could also be interpreted. Fig. 6.7A showed the cell-size boundary for the viable cell population. The cell size boundary was taken from the negative control. The number of events for viable cells withing the cell-size boundary was 9607 among the total events 10380 as could be observed from Table 6.2. This showed that alomst $94 \%$ of the cells were viable within the range. In Fig. 6.7B, it could be observed that the viable cells within the cell-size boundary exhibited high fluorescence signal after washing and this finding was well supported by the 
count of 9125 FITC-positive events among the 9607 total events from the viable cells. Therefore, this observation suggested that there was almost $90 \%$ of the viable cells within the cell-size boundary showed fluorescence positive signal inside cell after washing. On the other hand, similar observation was also noticed from the Fig. 6.7C and Table 6.2, where the number of events in Q1-1 exhibiting high positive fluorescence signal by the cells within the cell-size boundary was 9546, which was almost $92 \%$ of the total viable events. Therefore, combining these observations from the FACS results, it could be interpreted that there was a high increase of fluorescence signal (approx. 90\%) within the cell after washing. This in turn suggested a close 90\% cellular uptake efficiency for 5-FAM-HIV-1 TAT peptides in human adenocarcinoma cells A549. It was worth mentioning that the cut-off ranges of fluorescence that could be considered for a FITC-positive event was determined based on the fluorescence range for 5-FAM-HIV-1 TAT peptide shown in Fig. 6.7B $\left(\right.$ GFP-A $\left.=10^{3}-10^{5}\right)$. Since, the 5-FAM-HIV-1 TAT peptide was selected as the standard, so this cut off range was taken for all the other peptides.

\section{5(6)-FAM labeled $\beta^{3}$-Peptide (P9): FACS Results}

\begin{tabular}{|c|c|c|}
\hline Population & Events & \% Total \\
\hline All Events & 10326 & $\mathbf{1 0 0 . 0}$ \\
\hline Viable Events (P1) & 9651 & 93.5 \\
\hline FITC Positive & 2046 & 20.0 \\
\hline Q1-1 & 5240 & 50.7 \\
\hline Q2-1 & 142 & 1.4 \\
\hline Q3-1 & 4264 & 41.3 \\
\hline Q4-1 & 5 & 0.0 \\
\hline
\end{tabular}

Table 6.3: FACS Results for the 5(6)-FAM- $\beta^{3}$-Peptide (P9) At-A-Glance 


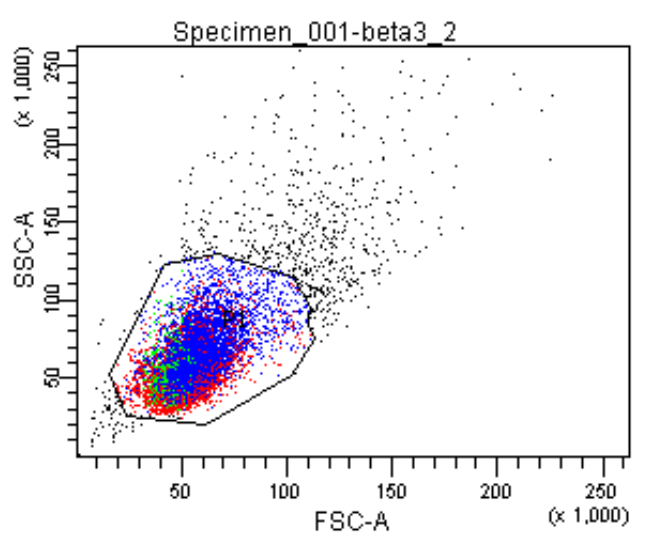

A

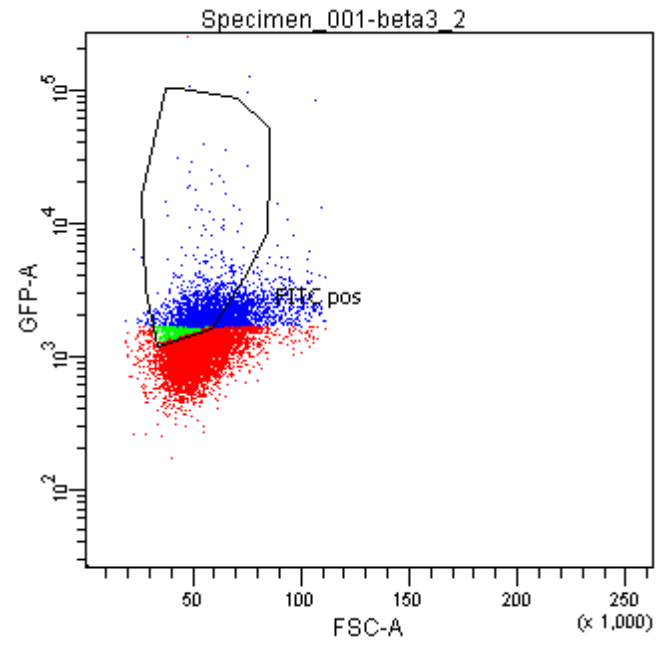

B

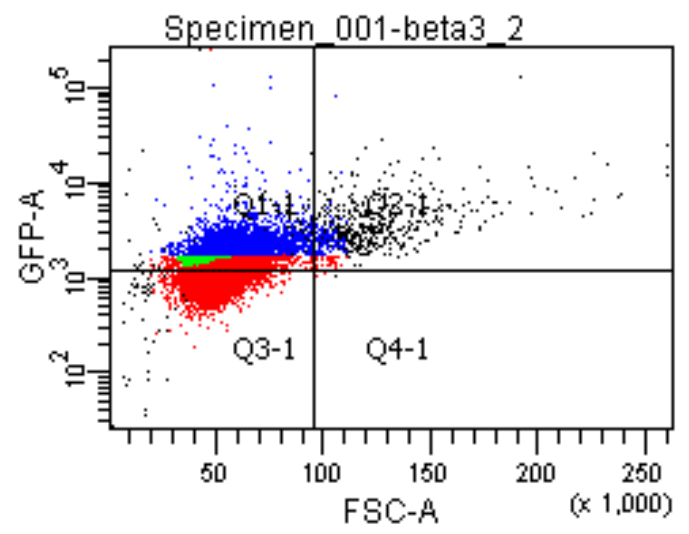

C

Figure 6.8: FACS results for 5(6)-FAM- $\beta^{3}$-Peptide (P9) treated human adenocarcinoma A549 cell line; cell-size vs viablity (A), cell size vs. fluorescence intensity (B) and quadrant divided plot of cell size vs. fluorescence intensity $(\boldsymbol{C})$

The Fig. 6.8A and Table 6.3 showed that the population of viable events within the cell-size boundary did not decrease and was kept at a high 93.5\%. This proved once again that the 5(6)FAM- $\beta$-Peptide (P9) was not cytotoxic and cellular viability was well maintained in its presence. It was quite evident from Fig. 6.8B and the Table 6.3 that there was much lower events recorded for viable cells within the cut-off positive fluorescence range. The FITC-positive event was reduced to only 20\%. It was also evident from Fig. 6.8C as well as Table 6.3 that the number of events in Q1-1, which was the only quandrant among the four quadrants where the events corresponded to viable single cell population within the cell-size boundary and positive fluorescence intensity cut-off, was only 50\%. Therefore, the combining results depicted that 
although the considerable positive fluorescence signal within the cells after washing was much reduced compared to the 5-FAM-HIV-1 TAT peptide, but there was definitely some significant cellular uptakes occuring for the 5(6)-FAM- $\beta^{3}$-Peptide (P9).

\section{5(6)-FAM labeled $\beta^{2} / \beta^{3}$-Peptide (P7): FACS Results}

\begin{tabular}{|c|c|c|}
\hline Population & Events & \% Total \\
\hline All Events & 10391 & 100.0 \\
\hline Viable Events (P1) & 9537 & 91.6 \\
\hline FITC Positive & 88 & 0.8 \\
\hline Q1-1 & 551 & 5.3 \\
\hline Q2-1 & 70 & 0.7 \\
\hline Q3-1 & $\mathbf{8 8 2 4}$ & 84.9 \\
\hline Q4-1 & 92 & 0.9 \\
\hline
\end{tabular}

Table 6.4: FACS Results for the 5(6)-FAM- $\beta^{2} / \beta^{3}$-Peptide (P7) At-A-Glance

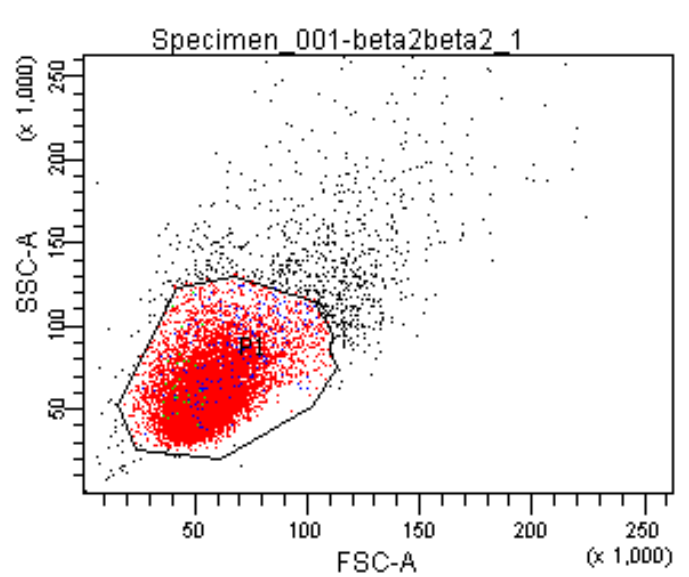

A

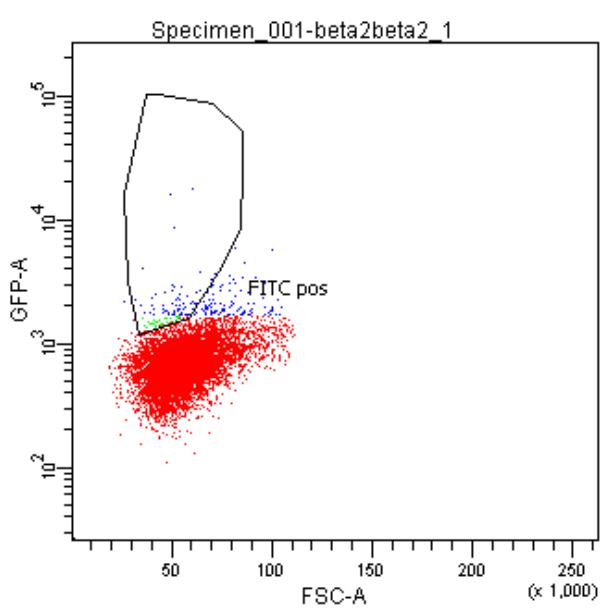

B 


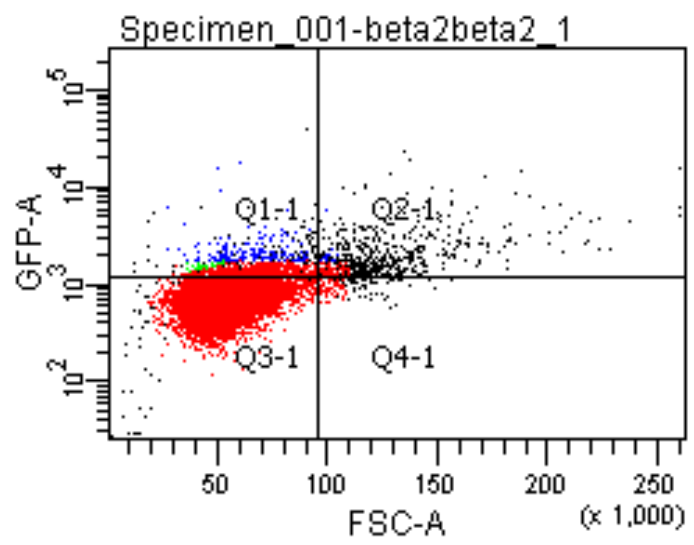

C

Figure 6.9: FACS results for 5(6)-FAM- $\beta^{2} / \beta^{3}$-Peptide (P7) treated human adenocarcinoma A549 cell line; cell-size vs viablity (A), cell size vs. fluorescence intensity (B) and quadrant divided plot of cell size vs. fluorescence intensity $(\boldsymbol{C})$

Just like the 5-FAM-HIV-1 TAT and the 5(6)-FAM- $\beta^{3}$-Peptide (P9), the 5(6)-FAM- $\beta^{2} / \beta^{3}$-peptide (P7) was also found to be completely non-cytotoxic as the viable events within the cell-size boundary was recorded to be almost 92\% (Figure 6.9A and Table 6.4). But unlike in the case of the 5(6)-FAM- $\beta^{3}$-Peptide (P9), the Fig. 6.9B and the Table 6.4 showed that FITC-positive events within the single cell-size boundary was only $0.8 \%$, which was almost negligible. Similary, the quadrant-divided plot in Fig. 6.9C as well the Table 6.4 showed that the positive fluorescence signal in the viable cells within the single cell-size boundary and the positive fluorescence intensity cut-off after washing was extremely low (approx. 5\%). These observations strongly suggested that unlike the 5(6)-FAM- $\beta^{3}$-Peptide (P9), there was almost no cellular uptake observed for the 5(6)-FAM- $\beta^{2} / \beta^{3}$-peptide (P7).

Therefore, from the FACS studies, it could be indicated that there was a stark difference between the cellular uptake efficiencies between the standard cell penetrating peptide, HIV-1 TAT peptide and the two different types of target $\beta$-peptides. 


\begin{tabular}{|l|c|c|c|c|c|c|c|c|}
\hline & $\begin{array}{c}\text { Control } \\
\text { Average }\end{array}$ & $\begin{array}{c}\text { Standard } \\
\text { Deviation }\end{array}$ & $\begin{array}{c}\text { HIV- } \\
\text { TAT } \\
\text { Average }\end{array}$ & $\begin{array}{c}\text { Standard } \\
\text { Deviation }\end{array}$ & $\begin{array}{c}\beta^{3-} \\
\text { Peptide } \\
\text { Average }\end{array}$ & $\begin{array}{c}\text { Standard } \\
\text { Deviation }\end{array}$ & $\begin{array}{c}\beta^{3} / \beta^{2}- \\
\text { Peptide } \\
\text { Average }\end{array}$ & $\begin{array}{c}\text { Standard } \\
\text { Deviation }\end{array}$ \\
\hline $\begin{array}{l}\text { Total } \\
\text { No. } \\
\text { Cells }\end{array}$ & 10323.5 & 46.5 & 10349 & 31.0 & 10348 & 22.0 & 10472.0 & 81.0 \\
\hline $\begin{array}{l}\text { Vital } \\
\text { Cells }\end{array}$ & 9698.0 & 4.0 & 9652.5 & 34.5 & 9651.5 & 0.5 & 9491.0 & 46.0 \\
\hline $\begin{array}{l}\text { No. } \\
\text { FITC } \\
\text { Post. } \\
\text { Cells }\end{array}$ & 30.0 & 3.0 & 9082.0 & 44.0 & 3154.0 & 146.0 & 167.0 & 29.0 \\
\hline $\begin{array}{l}\text { \% Vital } \\
\text { 93.94 }\end{array}$ & 0.46 & 93.27 & 0.05 & 93.2 & 0.19 & 90.64 & 1.14 \\
\hline $\begin{array}{l}\text { Positive } \\
\text { Cells }\end{array}$ & & & & & & & & \\
\hline
\end{tabular}

Table 6.5: Comparative FACS results for all three target pepides at-a-glance

The TAT peptide had a very high propensity to get internalized, perhaps mainly due to the presence of several positively charged amino acid residues, like arginines, whereas both the two $\beta$-peptides, being completely neutral and hydrophobic in nature, as expected, had much less capability for cellular uptake. But interestingly, there was also a distinct difference in cellular uptake efficiency between the two types of $\beta$-peptides. The $\beta^{3}$-peptide (P9) showed much higher cellular uptake efficiency (at least $20-30 \%$ of that of HIV-1 TAT peptide) than that of the $\beta^{2} / \beta^{3}$ peptide (P7). This finding indicated that although both the $\beta$-peptides were of similar lenghth and were composed of similar sequences, one showed much higher cellular uptake efficiency than the other. These differential cellular uptake efficiencies between two types of $\beta$-peptides could be contributed by the different secondary helical patterns. But prior to that, it was necessary to check whether this differences observed in FACS experiments were indeed statistically valid. In order to comment on whether the difference between the results of the two types of $\beta$-peptides were significant, the statistical method of Student's T-Test were performed on the available FACS data for the two petides. 


\section{Student's T-Test:}

Student's T-Test was a statistical method that compared two means to statistically verify if they were different from each other and how different they were. So, this test indicated how significant the difference between the two results were and whether the difference had occurred by chance.

There were two main parameters for this test, ' $\mathrm{T}$ '- and ' $\mathrm{P}$ '-values. The $\mathrm{T}$-value was the ratio between the difference between the two groups and difference within the two groups. The lasrger the T-score, the more differences were there between the two groups. Whereas the more important ' $P$ '-score was the probaility that the results from the sample data occurred by chance. The P-value could be from $0 \%$ to $100 \%$. The P-values were usually denoted in decimal forms. The lower the P-value, the lower was the probability that the difference between the results occurred by chance and hence the more significant was the difference in results. In most cases, a P-value of 0.05 (5\%) was accepted to be significant, as it indicated that there was only $5 \%$ probability that the difference in results occurred by chance.

Therefore, in order to ascertain whether the diference between the cellular uptakes between the two types of $\beta$-peptides observed from FACS experiments was significant and could be considered for further interpretation, the Student's T-Test was carried out. The result for the test came as, $\mathrm{P}=0.0254$. It statistically verified that there was only $2.5 \%$ probability that the difference in results between the two types of $\beta$-peptides for the cellular uptakes came by chance. Hence, the difference in results was statistically proven significant enough for further consideration and interpretation.

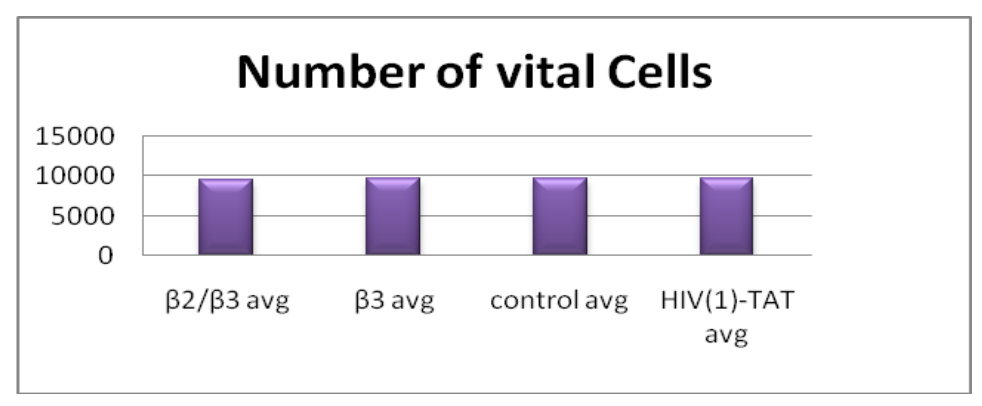




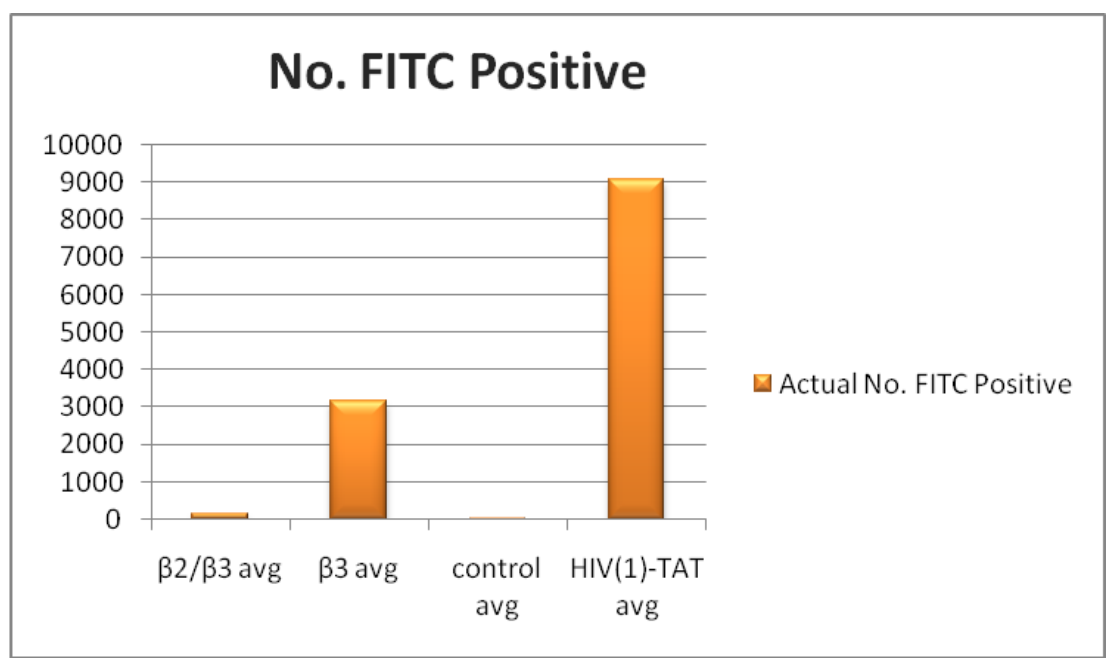

B

Figure 6.10: A comparative diagramatic illustration of the number of vital cells (A) and number of FITC-positive cells (B) for all the three target peptide and the negative control

It was promising to find that although being completely neutral in nature and composed of only hydrophobic amino acid residues, the $\beta^{3}$-peptide (P9) exhibited a possibility for significant cellular uptake. Besides, there was a stark difference in cellular uptake efficiencies between the two types of $\beta$-peptides, while both of the peptides were neutral, composed of completely hydrophobic amino acids and were of similar lengths. But from the FACS studies it could not specifically be ascertained whether all of the positive fluorescence signals recorded after washing were actually generated due to the proper penetration of the peptides through the plasma membrane into the intra-cellular media. Because similar positive fluorescence signals could also emerge if the peptides were somehow stuck to the membrane and not actually penetrated. In order to eliminate this possibility, confocal laser scanning fluorescence microscopy imaging was carried out to visualize the location of the peptides.

\subsubsection{Visualization: A Confocal Laser Scanning Fluorescence Microscopy Imaging Study}

The results for the cellular uptake study by FACS was further complemented by confocal laser scanning fluorescence microscopy imaging technique to confirm whether the positive fluorescence signal obtained by the FACS experiments were actually due to the internalization of 
the labeled peptides or some it was generated due to sticking of the peptides to the plasma membrane.

The imaging was carried out with freshly prepared FAM-labeled peptide samples and human bronchial adenocarcinoma A549 cell line. A set of imaging was performed imaging before the washing and another set after the washing. It was expected that after the washing, all the extracellular fluorescence would be completely quenched and only intra-cellular fluorescence would be visible fluorescent under the microscope. Therefore, initially a phase-contrast imaging was done to image the cells with their exact outlines with the normal white light laser. This was known as the brightfield imaging. Then the imaging was done in green channel that only showed the green fluorescence emitted by the fluorescent dyes as well as the fluorescently labeled peptides. This was called FITC. Next, another set of composite images were generated by combining the brightfield and FITC images for a better understanding of the location of the labeled peptides. All these images were taken before and after the washing for all the three target labeled peptides. Hence, the selected images for all the three peptides were depicted below.

\subsubsection{5-FAM-HIV-1 TAT Peptide}

\section{i) Before Washing:}

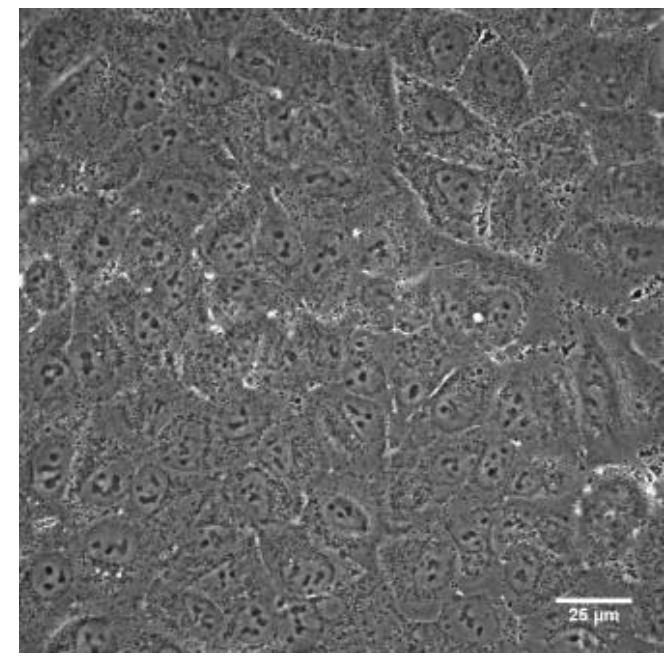

Phase-Contrast Brightfield Image

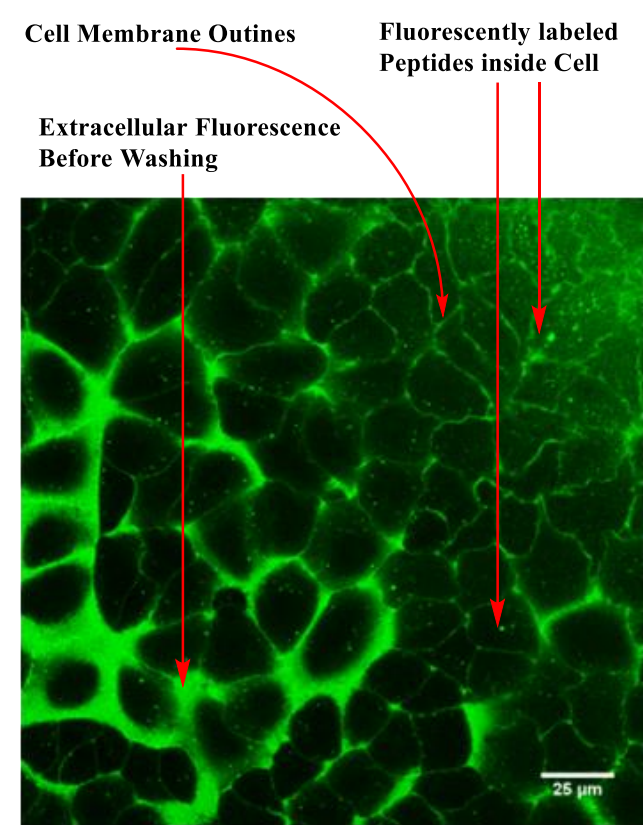

FITC Image 


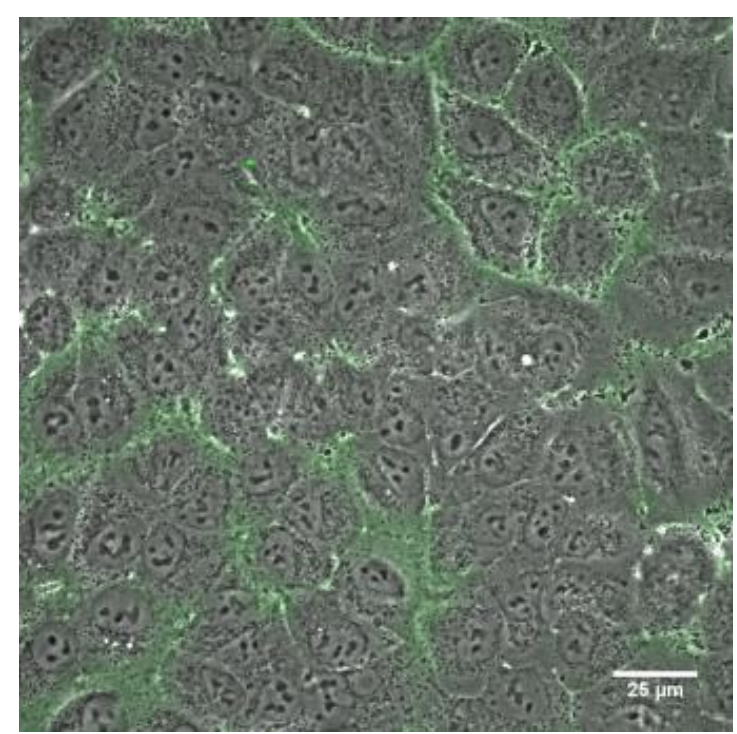

\section{Composite Image of Brightfield and FITC}

\section{ii) After Washing:}

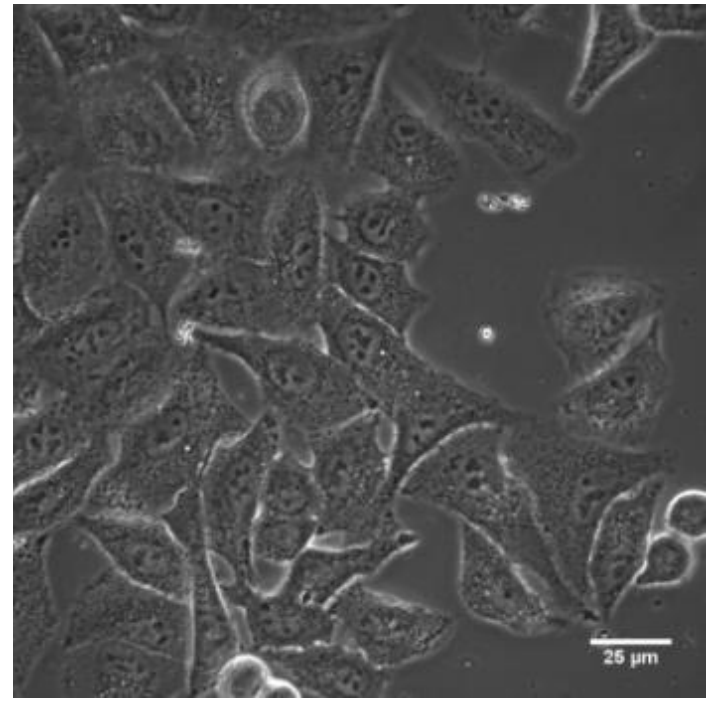

Phase-Contrast Brightfield Image
Intra-Cellular Fluorescent TAT Peptide after Washing

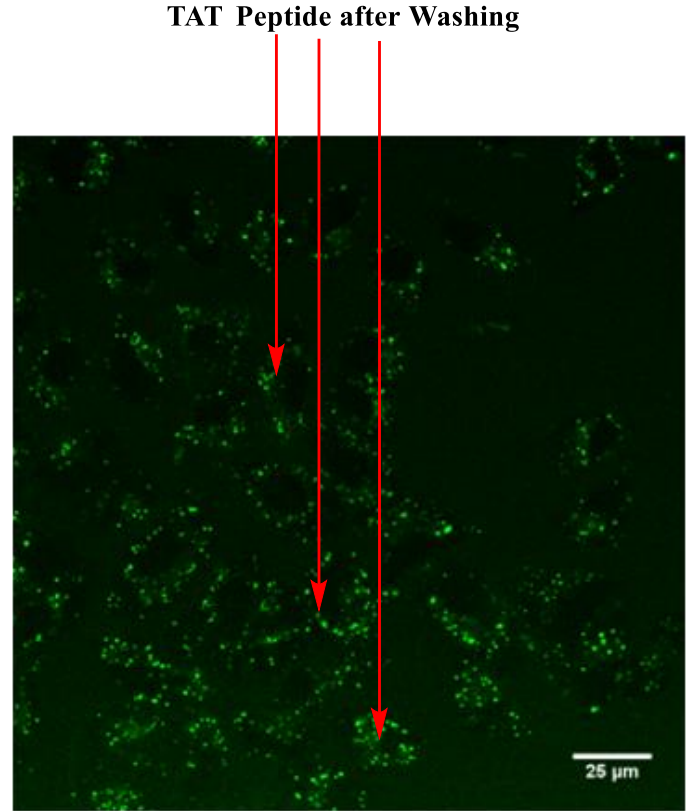

FITC Image 


\section{Cell Membrane Outlines}

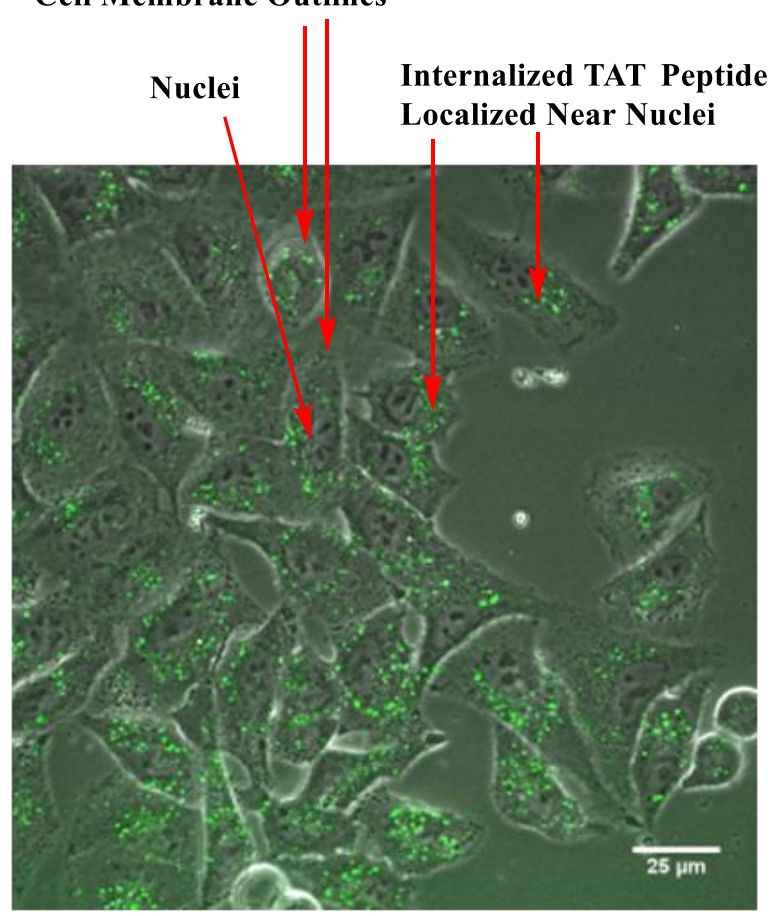

\section{Composite of Brightfield and FITC Images after Washing}

It was clearly seen that in case of 5-FAM-HIV-1 TAT peptide, the fluorescently labeled peptides were successfully internalized even before washing. In addition, after the washing and quenching of any extracellular fluorescence, strong fluorescence signal was observed in FACS experiments, which was well supported by the confocal laser scanning fluorescence microscopy images of the same pepide. After washing FITC image showed internalized fluorescent peptides and the composite image of the FITC and the brightfield distinctively showed the outlines of the plasma membrane and the exact location of the internalized peptides could be confirmed to be not stuck to the membrane but properly internalized into the intra-cellular environment. Moreover, it was also observed that the internalized peptides were localized at the cell nucleus.

\subsubsection{5(6)FAM-63-Peptide (P7)}




\section{i) Before Washing}

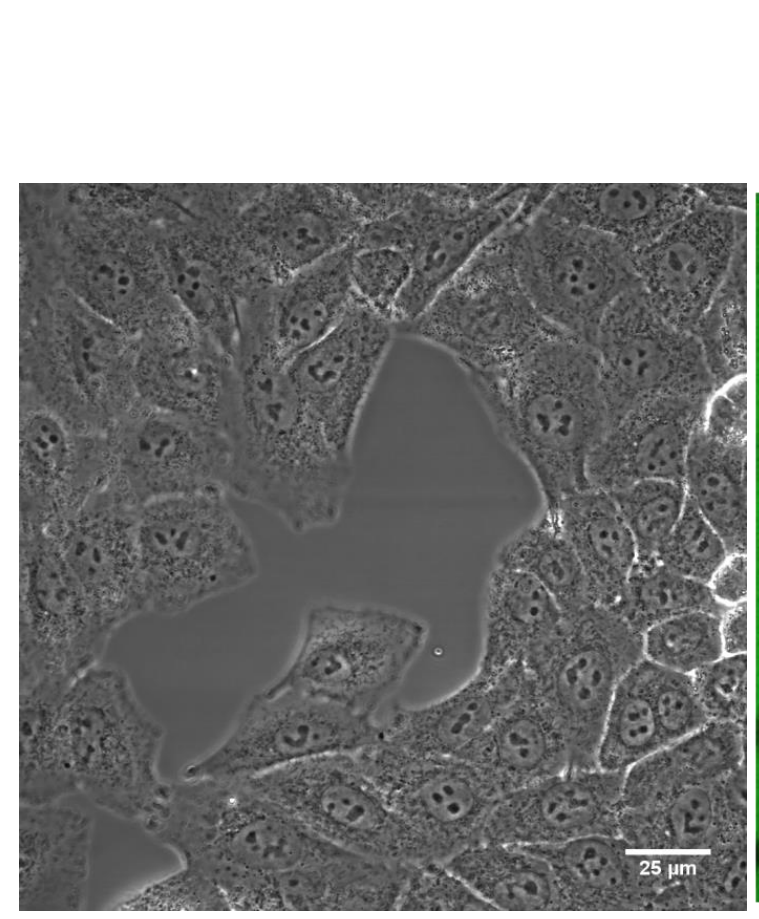

Phase-Contrast Brightfield Image

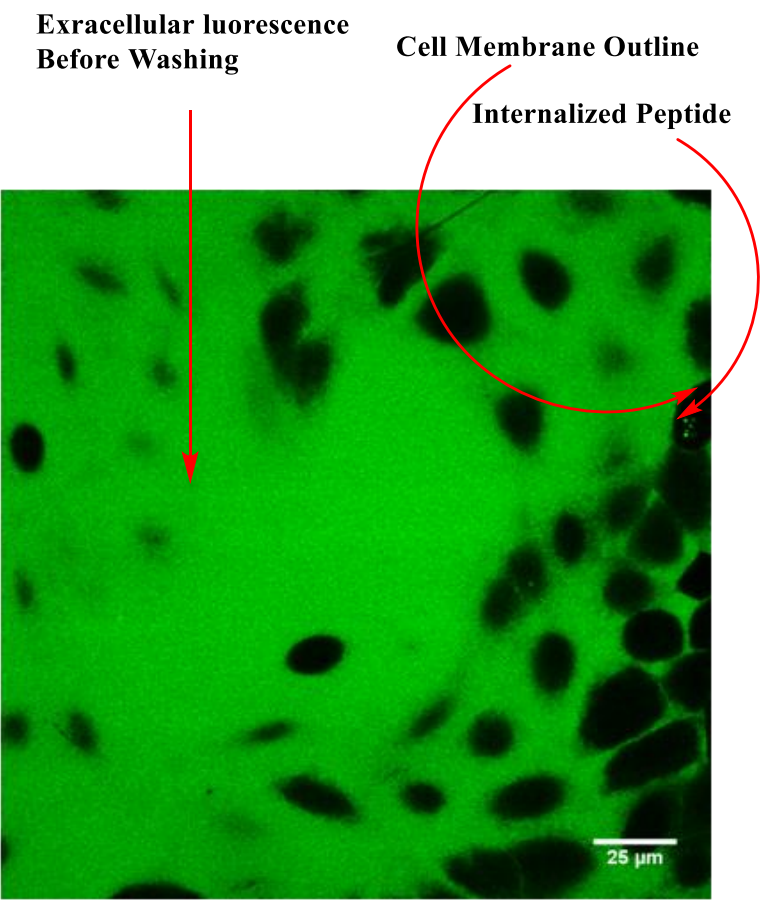

FITC Image

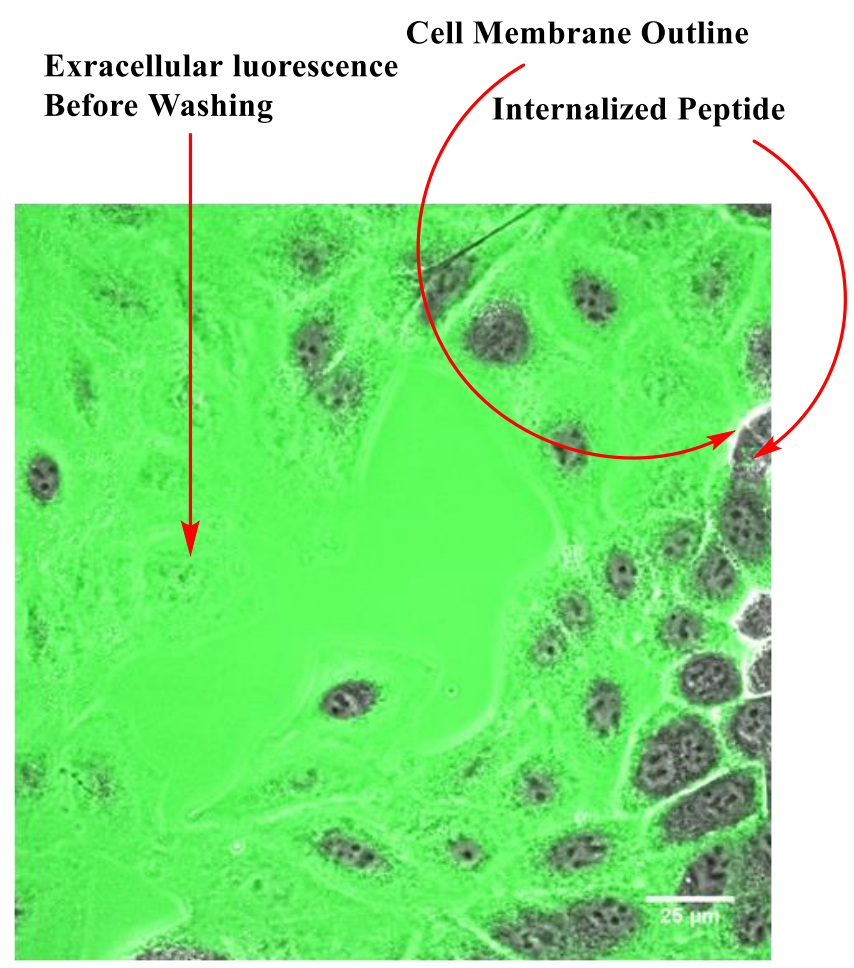

Composite of Brightfield and FITC Images before Washing 
ii) After Washing

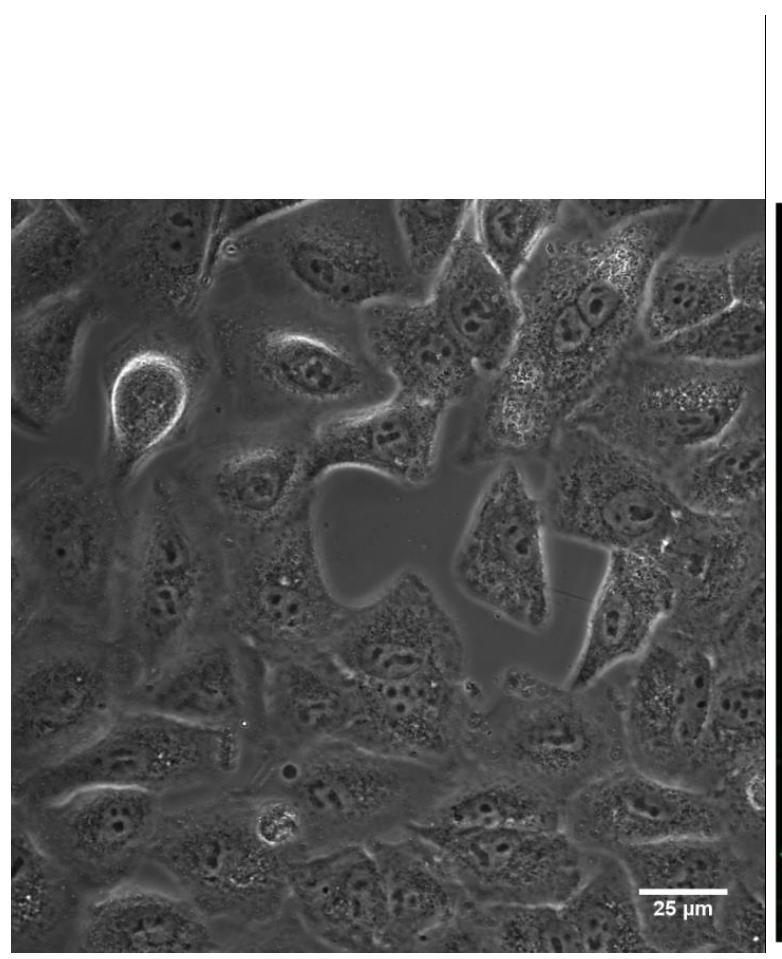

Phase-Contrast Brightfield Image

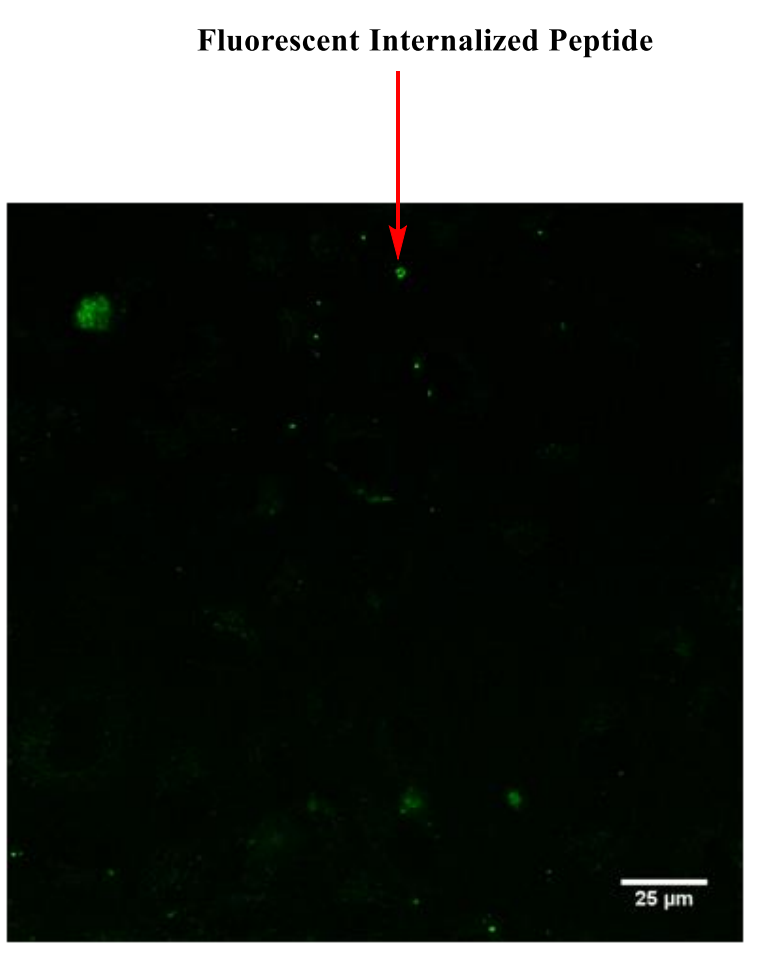

FITC Image

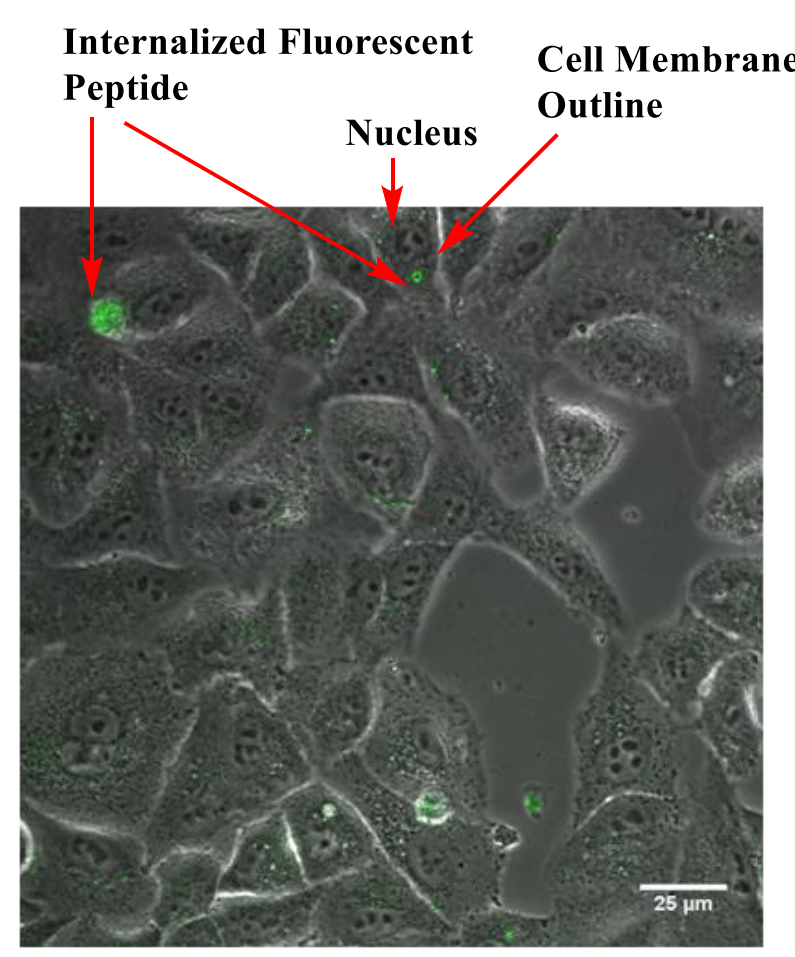

Composite of Brightfield and FITC Images 
The confocal laser scanning fluorescence microscopy images of the 5(6) FAM- $\beta^{3}$-peptide (P7) showed that although the extent of cellular uptake was reduced to $20-30 \%$ of that of the standard cell penetrating peptide, 5-FAM-HIV-1 TAT, but it clearly indicated that even for a neutral and completely hydrophobic short $\beta^{3}$-peptide sequence, there was considerable cellular uptake. Some fluorescent peptides could already been seen internalized into the cell before washing from the FITC image. Moreover, after washing and quenching of the extracellular fluorescence, there could be some green fluorescent peptide observed in FITC image and the proper location of the peptides could be ascertained to be inside the cell and definitely not stuck to the plasma membrane from the composite image, where the outline of the plasma membrane for each cell was clearly distinguished. It was further observed that like the TAT-peptide, the target $\beta^{3}$-Peptide also showed a tendency to get accummulated around the cellular nuclei.

\subsubsection{5(6)FAM-B²/B3-Peptide (P9)}

\section{i) Before Washing:}

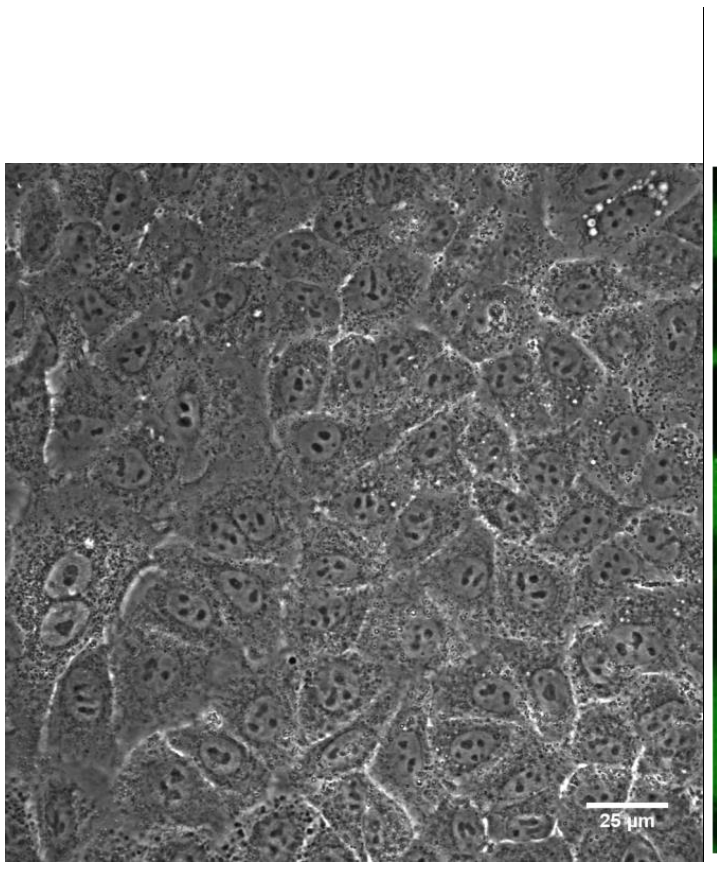

Phase-Contrast Brightfield Image

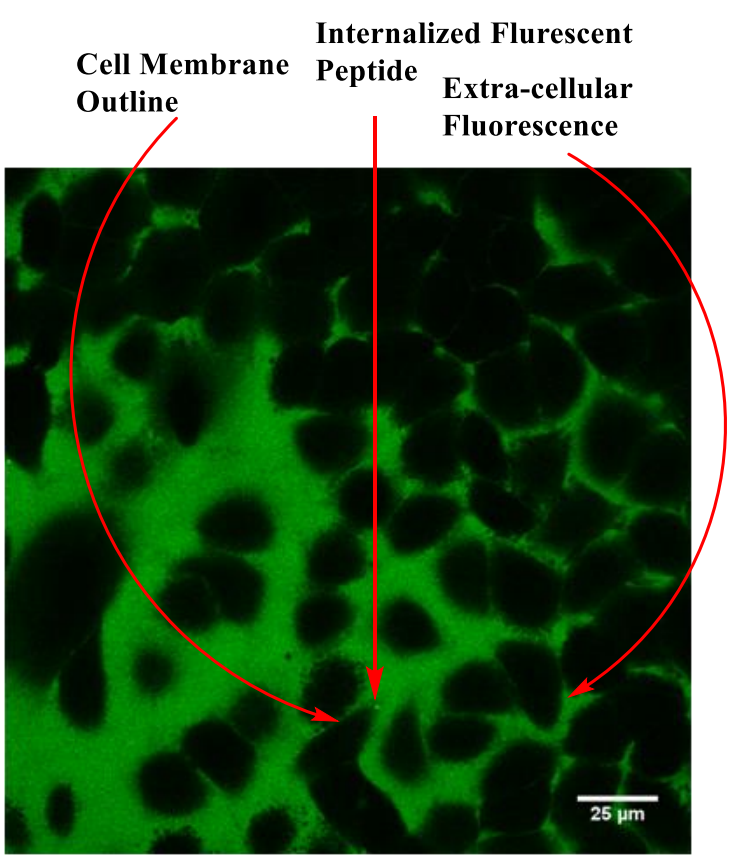

FITC Image 


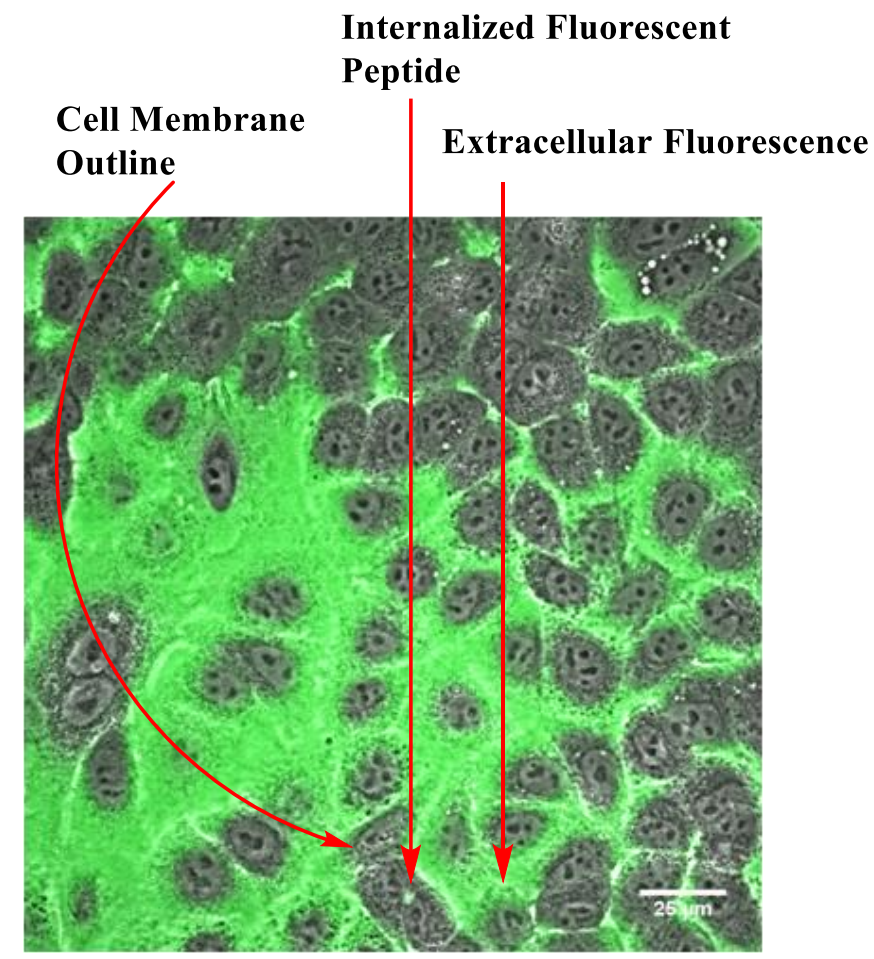

\section{Composite of Brightfield and FITC Images}

\section{ii) After Washing:}

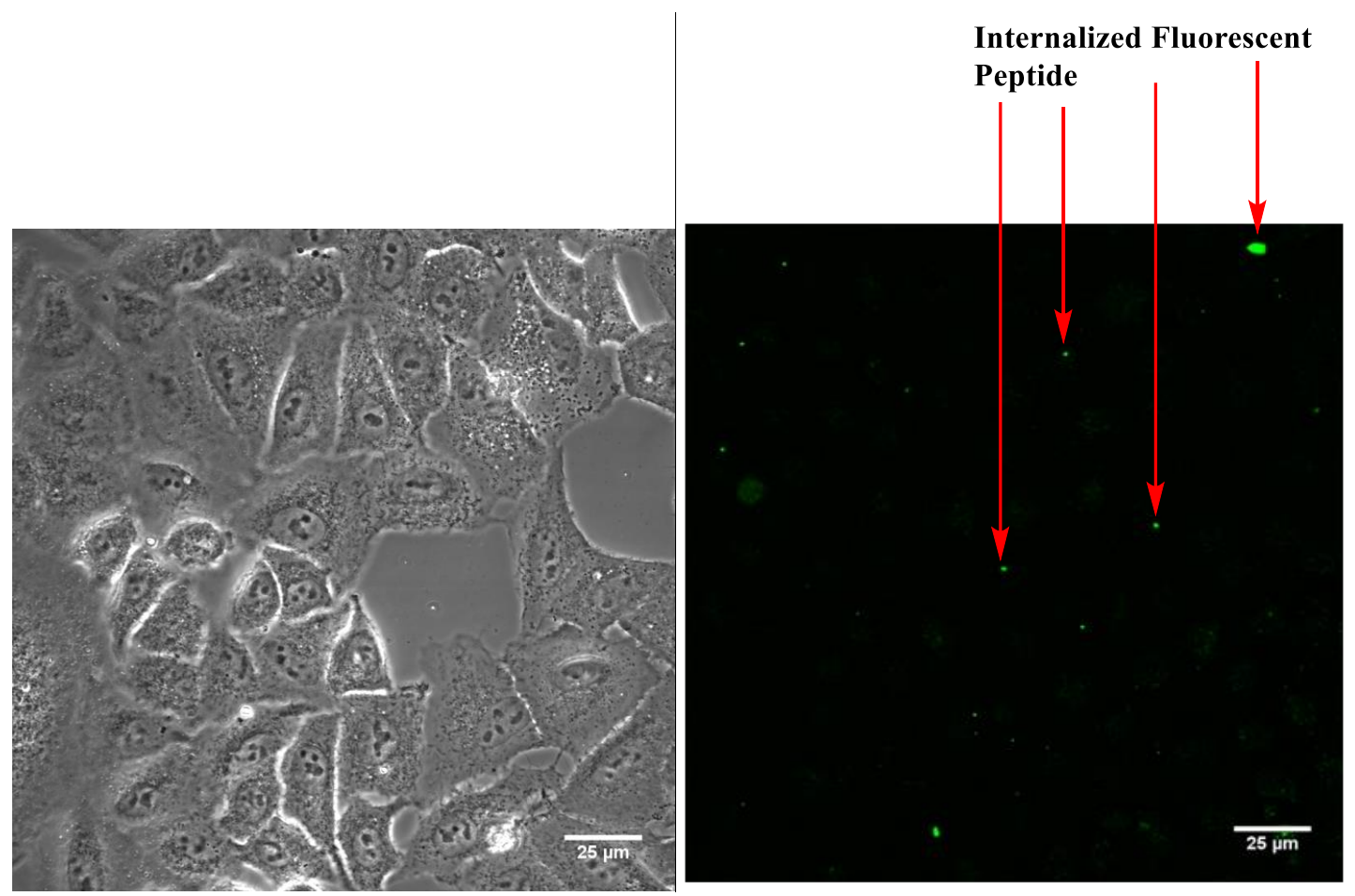

Phase-Contrast Brightfield Image

FITC Image 


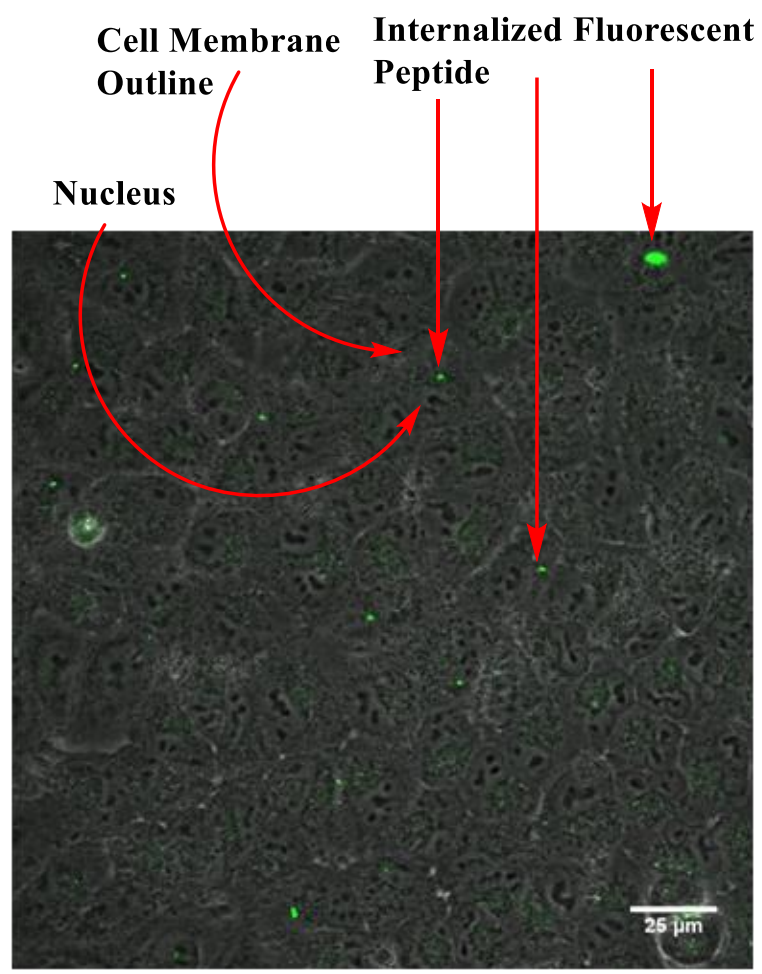

\section{Composite of Brightfield and FITC Images}

From the FITC and composite images before washing and quenching of extra-cellular fluorescence, some green fluorescent peptides could already been seen internalized into the cell. After the washing, all the extracellular fluorescence was observed to be quenched and the FITC image exhibited green fluorescent peptides at the intra-cellular environment. To make sure that the peptides indeed got properly internalized and were not stuck to the membrane, the composite image after washing confirmed it further, where the outlines of the plasma membrane for each cells were distinguishable and so the internalized target peptides were observed to be inside the cell and were accummulated near the cellular nuclei. Even compared to the target $\beta^{3}$. peptide, the visible internalized target $\beta^{2} / \beta^{3}$-peptide was extremely low under confocal laser scanning fluorescence microscopy imaging. This observation complemented the FACS results. 


\section{Conclusion}

It has long been known that peptides and proteins largely rely on cooperative effects to remain in their definite folded structures. ${ }^{[212-214]}$ An optimal balance between the charged interactions, hydrophobicity and most importantly hydrogen bonding is responsible for stable native folding of proteins. The intra-peptide hydrogen bonding can be dependent on an electrostatic dipoledipole interaction. The specific orientaton of the aminde dipoles for each residue leads to particular type of hydrogen bondings in a peptide chain that in turn becomes the guiding factor behind the formation of a specific type of folded secondary structure.[215] It has also long been predicted and proposed theoretically that besides forming and stabilizing secondary structures, the helical macro-dipole moment in a peptide chain also plays key role in other physico-chemical processes, like, transmembrane insertion and spanning[112] and dipole-induced selfassembly.[113] Therefore, it is well understood that helical macro-dipole moment in peptides acts as one of the more important regulating factors in several biochemical and physico-chemical processes. In the field of peptidomimetics, $\beta$-peptides have recently drawn much attenton and has proved to be one of the most promising synthetic mimics for the native $\alpha$-peptides. These non-natural polymers are known to fold into several types of stable helical secondary structures with as few as only four-six amino acids. On the other hand, these special mimics for native $\alpha$ peptides are also known to be stable towards degradation under the influence of proteolytic enzymes. [84, 216]

This study mainly focuses on the indirect experimental elucidation of any possible role of peptide helical macro-dipole moment on transmembrane insertion and spanning of different types of $\beta$-peptides. In order to shed light on whether the helical macro-dipole indeed has any significant role, along with the synthesis of 14-helical and 12-helical $\beta$-peptides with significant macro-dipole, the successful synthesis of a type of $\beta$-peptide that had almost zero macro-dipole moment, was one of the most important tasks. After long review, $\beta$-peptides composed of alternating $\beta^{2}$ and $\beta^{3}$-amino acids were found to fold into a rare 10/12-helical secondary structure. Unlike the 12-helix (12-membered H-honded rings), the dipole moments is 10-helix (10-mmbered H-bonded rings) were oriented perperndicular to the helical axis. Hence, alternate orientation of the macro-dipole in parallel and perpendicuar direction nullied the overall dipole moment, yielding a unique $\beta$-peptide with overall zero-helical macro-dipole moment. Now comparing the results of transmembrane insertion and spanning propensity between the $\beta$ peptides with macro-dipole and the one without gave a clear indication on whether the helical macro-dipole indeed had any function on these physico-chemical procedures. 
Two major groups of differet types of $\beta$-peptides were synthesized. In the first group, the bacterial protein Gramicidin A model inspired the sequence. The transmembrane $\beta$-peptide sequences were mainly composed of an alternate triad of $\beta^{3-}$ or $\beta^{2-D}$-(Val/Ala/Leu) amino acid sequences with at least one $\mathrm{D}$ - $\beta^{3}$-Trp residue at the $\mathrm{C}$-terminal to check transmembrane insertion by microenvironment polarity sensitive Trp-fluorescence. In the second group, 14helical peptides composed of D- $\beta^{3}$-Val with D- $\beta^{3}$-Trp followed by D- $\beta^{3}$-Lys towards the two termini and 12-helical peptides consisted of D- $\beta^{3}$-Leu with $\mathrm{D}-\beta^{3}$-Trp followed by D- $\beta^{3}$-Lys towards the two termini were synthesized. Two of this type of 14- and 12-helical peptides each were synthesized where one of the each types was longer and the other shorter in lengths. Since the macro-dipole is dependent of the sequence length, the change in peptide length would even strongly indicate whether any differences observed were indeed due to the difference in helical macro-dipole moment.

It is worth mentioning that the synthesis of all the enantio-pure $\beta$-amino acid building blocks had to be synthesized. The synthesis of $\beta^{3}$-amino acid building blocks were relatively simpl and could be accessed via Arndt-Eistert Homologation reaction starting with the corresponding $\alpha$-amino acid analogues. The yield were also above $75 \%$ on an average. But the synthesis of $\beta^{2}$-amino acid building blocks were extremely challenging, as unlike the $\beta^{3}$-amino acids, it could not be synthesized via Arndt-Eistert Homologation and different enantioselective strategis were adapted, including L-Proline catalyzed enantiselective aminomethylation of aldehydes and asymmetric $\alpha$-alkylation using $+(1 S, 2 S)$-Pseudoephedrine as chiral auxiliary. But each of the methods had series of synthetic disadvantages with overall very poor yield. After the synthesis of sufficient quantity of $\beta$-amino acid building blocks, the next challenging task was to synthesize the major target alternate 10/12-helical peptides composed of alternate $\beta^{3}$ - and $\beta^{2}$-amino acid residues. The synthesis of this type of transmembrane peptide sequences proved to even more challenging than that of the 14- or 12-helical peptides $\beta$-peptides. One reason could be the absence of overall helical macro-dipole moment made it even more hydrophobic in nature compared to the 14- or 12-helical peptides. The more hydrophobic the peptides were, the more was their tendency to self-aggregate and reduce the overall surface area in the resin while being synthesized by SPPS. Therefore, it already gave an indication during the synthesis that if the helical macro-dipole possessed such an effect on the hydrophobicity of the transmembrane peptides, it might also prove to be crucial to regulate other physico-chemical processes. After getting unsuccessful to synthesize the zero-dipole 10/12-helical target peptides in microwaveassisted SPPS and in heavy-fluorous-tagged solution phase synthesis strategy with elevated temperature, finally, a mixed strategy using non-microwave assisted manual SPPS at room 
temperature and microwave-assited manual SPPS at elevated temperature worked out to access the desired transmembrane peptides successfully with considerable yield.

Now the primary target was to investigate on whether the target peptides could be introduced inside large unilamellar vesicles of selected lipids. This was done using Trp-fluorescence spectroscopy. Trp-fluorescence is well known to be sensitive to the polarity of its microenvironment. Therefore, from the Trp-fluorescence emmision maxima of the peptides, it could be determined whether the Trp-residue was surrounded by a polar (aqueous), non-polar (hydrophobic/lipidic) or a membrane-water interface media. The Trp-fluorescence emission maxima changed according to the polarity of its microenvironment. In the begininning to check whether the different types of $\beta$-peptides could be inserted into the lipid bilayers, lipids were chosen so that the thickness of the lipid bilayers matched almost perfectly with the hydrophobic core length of the different types of $\beta$-peptides of comparable lengths. This was done to avoid any hydrophobic mismatch situation. Comparing the intrinsic Trp-fluorescence of the peptides in TRIS-buffer and in presence of lipid vesicles, it was observed that all the types of $\beta$-peptides were reconstituted into the lipid bilayers. Now the next target was to investigate on whether there was any difference in the ease of transmembrane insertion between the $\beta$-peptides with macrodipole moment (14- or 12-helix) and the one without a macro-dipole moment (10/12helix). Any difference in the tendency of transmembrane insertion between these two classes of $\beta$-peptides of similar length and sequences would indicate a possible role of the helical macrodipole moment in transmembrane insertion and spanning. To accomplish the target, two different lipids were chosen that had gradually increased bilayer thicknesses than that of the two types of $\beta$-peptides of similar transmembrane core lengths. So, there was a gradual increase in negative hydrophobic mismatches between the peptides and the two different lipids. It has been well known that EtOH has an ability to pertrub the lipid bilayer system in such a way that the lipids attain an interdigitated orientation and the midplane region is decreased. As a result, the bilayer thickness of the lipid becomes reduced. So, the idea was to find the minimum concentration $(\mathrm{v} / \mathrm{v})$ of EtOH required for each peptides for the minimum compensation of the negative hydrophobic mismatch by minimal reduction of the bilayer thickness of the lipids, so that the peptide could successfully be reconstituted into the membrane system. Now, any difference in the minimum concentration of EtOH required for reconstituting the two major types of $\beta$-peptides (with and without macro-dipole moment) of similar transmembrane lengths into the same lipid bilayer would indicate that one required more reduction of the bilayer thickness, i.e, more compenation of the negative hydrophobic mismatch than the other for successful transmembrane reconstitution and spanning. This would imply that one type was more prone towards transmembrane insertion than the other was. It was observed from the 
quantitattive estimation of EtOH induced negative hydrophobic mismatch compensation for successful reconstitution of transmembrane peptide segments into model lipid bilayers via polarity sensitive Trp-fluorescence spectroscopy that the 14-helical as well as 12-helical $\beta$ peptides with significant helical macro-dipole moment required much higher minimum concentration of EtOH, i.e, much higher compensation of negative hydrophobic mismatch than that of the alternate 10/12-helical transmembrane peptides with overall nullified macro-dipole moment. The lack of macro-dipole probably made this type of peptides more hydrophobic than any other $\beta$-peptide analogues and this extreme hydrophobicity helped it to interact with the hydrophobic lipidic core in a more energetically favourable manner. This led to the favourable transmembrane insertion and spanning capabilities of the zero-dipole alternate 10/12-helical $\beta$ peptides compared to the other types, such as, the 14- or 12-helices. This obsrvations experimentally indicated that not only the helical macro-dipole moment has a very important role in transmembrane peptide insertion and spanning but the zero-dipole peptides are perhaps the better candidate than that of the other types as model $\beta$-peptide based transmembrane domain systems.

To ensure that dipole moment was indeed responsible, similar experiments between 14-and 12helical long and short chain peptides were carried out. It has been well known that the longer the peptide chain, the higher the helical macro-dipole moment. So, in this experiment, all the other parameters, like the peptide sequences, conditions, etc, were kept constant and only the chain length was varied. As a result, it was observed that both in cases of 14- and 12-helical peptides, the longer the peptide chain length, the higher minimum concentration of EtOH, i.e, higher compensation of negative hydrophobic mismatch was required for reconstitution of the peptides into model lipid bilayer systems. Therefore, with the increase in chain length, the magnitude of helical macro-dipole moment also was increased proportionally and the less was the tendency to be reconstituted and spanned across the model lipid bilayer system. The result from these experiments also suggested that the helical macro-dipole could be one of the more important regulatory factors for transmembrane insertion and spanning of membrane peptides.

The next aim was to investigate on the secondary structures of the synthesized $\beta$-peptides. The secondary structures ere confirmed by CD-spectroscopic measurements. At first secondary structures were determined in aqueous solution (TRIS buffer) for all the peptides in question. The peptides showed their expected characteristic secondary structures in solution. Now the further task was to check on whether the respective helical secondary structures were maintained when inside lipid bilayer. It was observed that not only all the peptides maintained 
their native secondary helical structures inside lipid bilayers in case of hydrophobic matched peptide-lipid systems, but also they were also perfectly retained when the peptides were introduced inside lipid bilayer using minimum concentration of EtOH via negative hydrophobic mismatch compensation strategy. Therefore, the secondary structures of all the $\beta$-peptides were very stable in solution as well as in hydrophobic lipid environment. The stability of the helical secondary structures of the peptides were also tested against temperature starting from $20^{\circ} \mathrm{C}$ to as high as $80^{\circ} \mathrm{C}$ both aqueous solution and inside lipidic environment. In aqueous solution, it was found that even at high temperature as $80^{\circ} \mathrm{C}$, the 14 -helical $\beta$-peptides did not lose much of their helical contents, so were stable enough, whereas the alternate 10/12-helical $\beta$-peptides maintained their helicity perfectly upto $60^{\circ} \mathrm{C}$, but between $60^{\circ} \mathrm{C}-80^{\circ} \mathrm{C}$, it lost its helicity drastically. Hence, the 10/12-helical peptides were relatively unstable at temperature between $60^{\circ} \mathrm{C}-80^{\circ} \mathrm{C}$ in aqueous solution. Interestingly, when the peptides were inside lipidic environment, the 14-helical peptide almost uniformly held its helical secondary structure without losing much helicity, but surprisingly unlike in aquous solution, the 10/12-helical peptides did not lose its helicity betwen $60^{\circ} \mathrm{C}-80^{\circ} \mathrm{C}$, and rather were unformly stable as the 14 -helices. Therefore, it could be concluded from these observations that the 14-helical peptides were uniformly stable at high temperature as $80^{\circ} \mathrm{C}$ both in aqueous solution and in lipidic environment, while the alternate 10/12-helical peptides were unstable at temperature betwen $60^{\circ} \mathrm{C}-80^{\circ} \mathrm{C}$ in aqueous solution, but were very stable at this temperature range whn inside lipidic environment. So, in a way, the hydrophobic lipid core stabilized the 10/12-helical peptides more than that of the 14-helical peptides. This pronounced stability of the 10/12-helical peptides over the 14-helical ones in hydrophobic lipid environment could possibly be explained by the more hydrophobic nature of the 10/12-helices due to absence of any helical macro-dipole moment. The more hydrophobic peptides could interact more favourably with the hydrophobic lipid core and so were stabilized more than that of the other types of transmembrane peptides.

Therefore, it could now be verified experimentally that the helical macro-sipole moment indeed might have very important role in regulating transmembrane insertion and spanning of membrane proteins. The lower the magnitude of the helical macro-dipole in designed transmembrane domains, the higher the tendency for membrane insertion and spanning and higher is the stability inside hydrophobic lipidic core environment. Keeping the important role of helical macro-dipole moment in mind, the further design of future $\beta$-peptide based model transmembrane domain systems could be more projected towards the alternate 10/12-helical peptides. 
Due to the effective stability of the $\beta$-peptides against proteolytic degradation in presence of protease enzymes, these synthetic class of peptides capable of mimicking the functions of native $\alpha$-peptides were a promising candidate as cell penetrating peptides. The two types (14-helical and 10/12-helical) of short chain $\beta$-peptides were tested to check on whether they possessed any cell penetrating capabilities at all. Almost all the reported cell-penetrating peptides contain multiple positive charges in it that act as an important tool to create membrane pores for direct tranlocation across the plama membrane. But the positive charges show severe physiological adverse-effects in long run via gradual accummulation into different organs, like liver. So, the target $\beta$-peptides were designed to adress two main disadvantages of conventional cell penetrating peptides, i.e., prone towards enzymatic degradation in presence of proteases and the multiple positively charges. Since, the designed peptides were completely neutal hydrophobic in nature, so it was quite unlikely that they would succeed in translocating across membrane and would show some significant cellular uptake at all. A historically known cell penetrating peptide, HIV-1 TAT, was used as a standard for the two types of target $\beta$-peptides.

The target peptides were tested for any potent cytotoxic effect against the human bronchial adenocarcinoma cell lines (A549) and no cytotoxicity was observed by clonogenic and MTT assay upto $15 \mu \mathrm{M}$ peptide cncentrations. Thereafter, the cellular uptake was quantitatively measured by fluorescence-activated cell sorting (FACS), a special type of flow cytometry technique using the same cell line. It was found that most importantly both the types of $\beta$ peptides showed visible positive fluorescence increase signal into cells after washing indicating a considerable cellular uptake. But more surprisingly, there was a big difference in cellular uptake efficiencies between the two types of $\beta$-peptides. The 14-helical $\beta^{3}$-peptide showed almost $30 \%$ uptake compared to HIV-1 TAT whereas the alternate 10/12-helical $\beta^{2} / \beta^{3}$-peptide showed only $1 \%$. The difference in cellular uptake efficiency between the two types of $\beta$-peptides was statistically verified to be significant enough with only $2.5 \%$ probable chance that the experimental difference came by chance. The peptides were also imaged with confocal laser scanning fluorescence microscopy to strongly propose that the peptide were indeed internalized into the cell and were not stuck to the plasma membrane.

It was evident from the experiments that the target $\beta$-peptides could be internalized into cell even when they were completely neutral and hydrophobic with their added stability against proteolytic degradation. Especially the 14-helical $\beta$-peptides composed of D- $\beta^{3}$-amino acids were more promising as candidates for cell penetrating peptide design. With further modifications of the sequences, the cellular uptake efficiencies could possibly be increased. Therefore, the $\beta$ - 
peptides were found to be a promising target candidate to design neutral and proteolytically stable cell penetrating peptides.

On the other hand, the stark differences in cellular uptake efficiencies between the two types of $\beta$-peptides indicated that similar to the transmembrane insertion, the cellular uptake efficiency could also be regulated by the helical macro-dipole moment of the target peptides. In this case, the 14-helical $\beta^{3}$-peptides with significant macro-dipole moment exhibited much higher cellular uptake than that of the alternate 10/12-helical $\beta^{2} / \beta^{3}$-peptides with nullified macro-dipole. Both the target peptides were of similar length and were composed of similar amino acid sequences. So, the major difference between the two peptides were their secondary helical structures, in which one of them possessed significant helical macro-dipole and the other almost completely lacked in it. Therefore, any differencial uptake efficiency attributed towards the major structural difference betwen them, i.e., the presence and absence of helical macro-dipole moment due to their different secondary helical patterns. Although both the peptides were completely neutral and hydrophobic, but the overall helical macro-dipole moment created a partial charged character in 14-helical $\beta^{3}$-peptide, making it slightly more hydrophilic in nature. Since it has already been discussed that the positive charged character played a crucial role in internalization of the peptides across plasma membrane via direct translocation, so it could be assumed that the partial charged character due to helical-dipole moment in 14-helical peptides was one of the major guiding factor that induced higher cellular uptake efficiency. While the 10/12-helical alternate $\beta^{2} / \beta^{3}$-peptides completely lacked in helical-dipole moment and so had no partial charged character in it. The absence of this charged character might have played a key role in reduced cellular uptake potential of this type of peptides. Since the difference in uptake results between the two $\beta$-peptides were statistically verified to be significant, so it could ultimately be propsed that the higher the magnitude of helical macro-dipole moment in peptides, the higher its potential for cellula uptake and internalization.

In conclusion, the helical macro-dipole moment was found to be really significant in several physico-chemical processes, including membrane insertion, spanning and cellular uptake, penetration. Interestingly, the effect of macro-dipole moment was found to be opposite for these two processes. In case of designing, $\beta$-peptide based artificial transmembrane domain systems capable of membrane insertion and spanning, peptides with lower magnitude of helical macrodipole moment was more promising, whereas in case formulating $\beta$-peptide based novel cell penetrating peptides, peptides with significant magnitude of helical macro-dipole moment was more potent. These important experimental observation and results could be extremely important both for future development in the field of designing $\beta$-peptide based artificial 
membrane channels as well as in formulation of novel cell penetrating peptides as vectors to deliver target cell impermeable cargo molecules within the cells that would potentially be proteolytically stable and free of accummulation related physiological sie effects.

\section{Experimental Section:}

\subsection{General}

\section{Solvents}

The dry solvents, such as, THF, DMF, DCM, MeOH, iPrOH and $\mathrm{CHCl}_{3}$ were purchased from Sigma Aldrich (Taufkirchen, Germany) that were stored over molecular sieves (4Å). All the technical grade solvents were distilled and purified prior using. Some solvents like, EtOH, pentane, dodecane, pyridine, 1,4-dioxane, toluene, EtOAc, DCM, MeOH, DMF, NMP, TFE, diethyl ether, acetonitrile $\mathrm{CHCl}_{3}$, were available in highest purity and were used without further purification. These solvents were obtained from GL Biochem, TCI, Alfa Aesar, Iris Biotech GmbH, Carl Roth Ltd, VWR International Ltd, Fischer Scientific, Merck and Sigma Aldrich. Piperidine was purchased from Riedel de Haen and used without further purification. For HPLC purification, Acetonitrile and $\mathrm{MeOH}$ were available as HPLC-grade and ultra-pure water (Mili-Q-Water) was obtained using a water purification system Simplicity (Millipore, Bredford, UK).

\section{Reagents}

All reagents were of highest grade and used in the experiments as supplied. The protected amino acids derivatives, HIV-1 TAT peptides, coupling reagents, other reagents, catalysts, fluporophores and resins for solid phase peptide synthesis (SPPS) were purchased from Sigma Aldrich (Taufkirhen, Germany), Bachem AG (Heidelberg, Germany), VWR International (Fontenay-sous-Bios, France), ABCR (Karlsruhe, Germany), Merch (Darmstadt, Germany), Alfa Aesar (Karlsruhe, Germany), Acros Organics (Geel, Belgium), TCI (Zwijndrecht, Belgium), Grüssing GmbH (Filsum, Germany), Fluorochem (Hadfield, UK), GL Biochem (Shanghai, China), 
Carl Roth GmbH (Karlsruhe, Germany), ABCR (Karlsruhe, Germany) and Polypeptide Group (Strassbourg, France). The fluorinated compounds, such as heavy fluorous tags, fluorinated silica gel 40, were purchased from Fluoroflash ${ }^{\circledR}$ (Fluorous Technologies Incorporated, Ambridge, USA). The lipids were purchased from Avanti Polar Lipids (Alabaster, USA), Sigma Aldrich (St. Louis, USA) and Bachem (Bubendorf, Switzerland).

\section{Reactions}

Air and/or moisture sensitive reactions were carried out under argon atmosphere using Schlenk technique. The glass apparatus used for the reactions were initially heated up with a heat-gun under inert atmosphere, dried completely under vacuum, and at last flushed with argon (5 $\times$ ).

\section{Lyophilization}

Christ Alpha-2-4 Lyophilizer (Osterode am Harz, Germany) equipped with a high-vacuum pump was used to freeze-dry (lyophilize) the building blocks and the fractions of HPLC-purified peptides in aqueous solutions containing minor proportions of acetonitrile or dioxane. For smaller fractions stored in Eppendorf vials an evacuable Christ RVC 2-18 or Christ RVC 2-18 CD Plus centrifuge connected to the lyophilizer was applied.

\section{Storage}

Amino acid building blocks, protected amino acid derivatives, peptides, lipids and some of the asymmetric NHC-carbene catalysts were stored as solids at $-22^{\circ} \mathrm{C}$ in the freezer. The SPPS-resins were stored at $4-8^{\circ} \mathrm{C}$ inside normal refrigerator. The chemicals were warmed to room temperature prior to use.

\section{Chromatography Techniques}

\section{a. Thin Layer Chromatography (TLC)}

Analytical thin-layer chromatography (TLC) was carried out on Merck (Darmstadt, Germany) silica gel $60 \mathrm{~F}_{254}$ aluminium plates. The detection of the spots were done either under UV 
radiation at $254 \mathrm{~nm}$ (in case of UV-active compounds) or dipping into a solution of ninhydrin (2.0 g ninhydrin, $1 \mathrm{~mL} \mathrm{AcOH}$ and $100 \mathrm{~mL} \mathrm{EtOH)} \mathrm{or} \mathrm{KMnO}_{4}(1.0 \mathrm{~g} \mathrm{KMnO}$, $1.5 \mathrm{~mL}$ \% aq. NaOH, $6.75 \mathrm{~g} \mathrm{~K}_{2} \mathrm{CO}_{3}$ and $100 \mathrm{~mL} \mathrm{EtOH}$ ) followed by heating to detect amines.

\section{b. Flash Column Chromatography}

Flash column chromatography was carried out using Merck silica gel 60 (40-60 $\mu \mathrm{m})$ under reduced pressure (01.-1 bar) to facilitate the process with increased efficacy. In some cases, the column chromatography has been performed only under gravitational force without applying reduced pressure. All solid compound were dissolved and adsorbed to the silica gel (50.100 times weight excess compared to the weight of the loaded sample) filled inside the fritted glass column.

\section{c. Fluorinated Solid Phase Extraction (FSPE)}

Fluorinated solid phase extraction was done especially to purify heavy fluorous-tagged peptides from the non-fluorinated impurities after each coupling steps in solution phase peptide synthesis. Fluorinated silica gel $40(40 \mu \mathrm{m})$ from Fluorous Technologies Incorportaed (Ambridge, USA) is used in this purpose. The impure sample was loaded into the fritted glass syringes and adsorbed to the fluorinated silica gel.

\section{d. High Performance Liquid Chromatography}

Reversed Phase HPLC: The crude peptides were further purified using reversed phase (RP)HPLC systems (ÄKTA BASIC 900, pump type P900, variable wavelength detector UV-900 and JASCO-FC-2088-30, pump type PU-2086 plus, multiwavelength detector MD 2010 plus) from Amersham Pharmacia Biotech(Freiburg, Germany) and JASCO Deutschland GmbH (Pfungstadt, Germany), respectively. The UV-absorption was detected at $215 \mathrm{~nm}, 254 \mathrm{~nm}$ and $280 \mathrm{~nm}$ in case of non-labeled peptides, whereas for NBD and 5(6)-Carboxyfluorescein labeled peptide derivatives, the UV-absorptions were recorded at $464 \mathrm{~nm}$ and $490 \mathrm{~nm}$, respectively instead of the detection at $254 \mathrm{~nm}$. The crude samples were dissolved in $\mathrm{MeOH} / \mathrm{H}_{2} \mathrm{O}$ and filtered prior to use in HPLC. The following C-18 columns were used for the purification of the crude peptides with a linear gradient of $\mathrm{A}$ (water $+0.1 \% \mathrm{TFA})$ to $\mathrm{B}(\mathrm{MeCN}+0.08 \% \mathrm{TFA})$ 
- Analytical:

- Semi-preparative:
MN Nucleodur ${ }^{\circledR} 100-5-C 18,250 \mathrm{~mm} \times 4.6 \mathrm{~mm}, 5 \mu \mathrm{m}$, flow rate: $1 \mathrm{~mL} / \mathrm{min}$

MN Nucleodur ${ }^{\circledR} 100-5-C 18,250 \mathrm{~mm} \times 10 \mathrm{~mm}, 5 \mu \mathrm{m}$, flow rate: $3 \mathrm{~mL} / \mathrm{min}$

MN Nucleodur ${ }^{\circledR} 100-5-C 18,250 \mathrm{~mm} \times 21 \mathrm{~mm}, 5 \mu \mathrm{m}$, flow rate: $10 \mathrm{~mL} / \mathrm{min}$.

Normal Phase (Chiral) HPLC: The enantiopurity of the intermediate products of NHC-carbene asymmetric catalysis was determined using analytical HPLC system (JASCO LC.NetII/ADC, binary pump type Jasco PU-2080 plus, PDA wavelength detector MD-2010 plus and AS-2055 plus intelligent sampler). The UV-absorption was detected at $215 \mathrm{~nm}, 254 \mathrm{~nm}$ and $280 \mathrm{~nm}$. The crude samples were dissolved in $\mathrm{PrOH} / \mathrm{Hexane}$ and filtered prior to use. The following chiral columns were used to determine the enantiopurity of the intermediate products with a linear gradient of C (iPrOH) to D (n-Hexane):

- Analytical:

i) Daicel ChiralPak ${ }^{\circledR}$ IA Immobilized chiral column, $250 \times 4.6,5 \mu \mathrm{m}$, flow rate: $1 \mathrm{~mL} / \mathrm{min}$

ii) Daicel ChiralPak ${ }^{\circledR}$ IB Immobilized chiral column, $250 \times 4.6,5 \mu \mathrm{m}$, flow rate: $1 \mathrm{~mL} / \mathrm{min}$

iii) Daicel ChiralPak ${ }^{\circledR}$ OD-H Normal phase chiral column, $250 \times 4.6,5 \mu \mathrm{m}$, flow rate: $1 \mathrm{~mL} / \mathrm{min}$

- Semi-preparative:

i) Daicel ChiralPak ${ }^{\circledR}$ IA Immobilized chiral column, $250 \times 10.0,5 \mu \mathrm{m}$, flow rate: $3 \mathrm{~mL} / \mathrm{min}$

ii) Daicel ChiralPak ${ }^{\circledR}$ OD-H Normal phase chiral column, $250 \times 10.0,5 \mu \mathrm{m}$, flow rate: $3 \mathrm{~mL} / \mathrm{min}$

\subsection{Characterization}

\subsubsection{Nuclear Magnetic Resonance Spectroscopy (NMR)}

The ${ }^{1} \mathrm{H}$ - and ${ }^{13} \mathrm{C}-\mathrm{NMR}$ spectra were recorded with Varian spectrometers (Unity 300 and Inova 500). The chemical shifts $(\delta)$ are denoted in parts per million (ppm) downfield of TMS. The resonance of the residual areas of different deuterated solvents were used as internal standards. The sample temperature was ambient for $\mathrm{CD}_{3} \mathrm{OD}$ and $308 \mathrm{~K}$ for DMSO- $\mathrm{D}_{6}$. Abbreviations for 
multiplicities are 's' - singlet; ' $d$ ' - doublet; ' $t$ ' - triplet; ' $q$ ' - quartret; 'm' - multiplet and 'br' broad. The coupling constants are quoted as ' ${ }^{\prime} J_{x y}$ ' in Hertz (Hz), where ' $n$ ' is the order of coupling between ' $x$ ' and ' $y$ '.

\subsubsection{Mass Spectrometry}

The electrospray-ionization (ESI) and high-resolution ESI mass spectra (HRMS-ESI) were recorded with Bruker devices (MicroTOF or maXis).

\subsubsection{Fluorescence Spectrometry}

Fluorescence spectra were obtained using Jasco FP 6200 and Jasco FP 8500 (Groß-Umstadt, Germany) under temperature control with Jasco thermostat (ETC-272T) and Julabo thermostat (F250), respectively.

The excitation wavelength for the detection of tryptophan fluorescence emission spectra was set at $280 \mathrm{~nm}$ and the fluorescence emission was detected between 290-500 $\mathrm{nm}$ with a micro quartz glass precision cell Suprasil ${ }^{\circledR}$ (type: 115F-QS, 10mm). The tryptophan fluorescence was measured either in TRIS buffer or within large unilamellar vesicles of DLPC, DMPC, POPC, 22:1 (cis) PC and 24:1 (cis) PC (peptide to lipid ration kept constant at 1:50). The excitation- and emission-bandwidths were set to $5.0 \mathrm{~nm}$, the response was 'fast', the data pitch was $1.0 \mathrm{~nm}$; the sensitivity was kept 'medium', the scanning speed was $100 \mathrm{~nm} / \mathrm{min}$ to $150 \mathrm{~nm} / \mathrm{min}$, and the temperature of measurement was varied depending on the transition temperatures of the lipids (from $25^{\circ} \mathrm{C}$ to $50^{\circ} \mathrm{C}$ ). The concentration of the peptide was kept at $12 \mu \mathrm{M}$.

To record the 5(6)-Carboxyfluorescein fluorescence, the excitation wavelength was set to 494 $\mathrm{nm}$ and fluorescence emission was obtained between 450-650 nm with a micro quartz glass precision cell Suprasil ${ }^{\circledR}$ (type: 115F-QS, 10mm). The excitation- and emission-bandwidths were set to $5.0 \mathrm{~nm}$, the data pitch was $1.0 \mathrm{~nm}$, the response was 'fast', the sensitivity was 'medium' or 'high', the scanning speed was set to $100 \mathrm{~nm} / \mathrm{min}$, and the temperature was varied depending on the transition temperatures of the lipids (from $25^{\circ} \mathrm{C}$ to $50^{\circ} \mathrm{C}$ ). Special care was taken to exclude any contact of light to the sample due to high photosensitivity of the fluorophore. The labeledpeptide concentration was maintained constant at $12 \mu \mathrm{M}$.

\subsubsection{UV/VIS Spectroscopy}

The UV spectra at fixed wavelength was recorded to accurately estimate the loading density of the first amino acid to the resin or to obtain the peptide concentration using Lambert-Beer's Law: 


$$
C=\frac{A}{\varepsilon \cdot 1}
$$

Where $\boldsymbol{C}$ is the concentration of the target compound ( $\left.\mathrm{mol} \mathrm{L}^{-1}\right)$

$\boldsymbol{A}$ is the absorbance

$\boldsymbol{\varepsilon}$ is the molar absorptivity (Lit. $\mathrm{mol}^{-1} \mathrm{~cm}^{-1}$ )

$\boldsymbol{I}$ is the path length of the cuvette containing the sample $(\mathrm{cm})$

Concentration of the stock solution was measured with Thermo Scientific NanoDrop 2000c spectrophotometer at $25^{\circ} \mathrm{C}$.

The molar absorptivity of a single tryptophan at $280 \mathrm{~nm}$ excitation wavelength was taken as $\boldsymbol{\varepsilon}_{\mathbf{2 8 0}}$ $=5690 \mathrm{M}^{-1} \mathrm{~cm}^{-1}$, whereas that of a single 5(6)-Carboxyfluorescein at $494 \mathrm{~nm}$ excitation

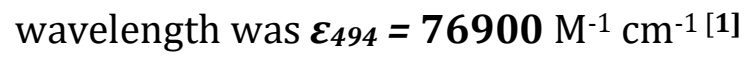

\subsubsection{Estimation of loading density}

The resin loading was estimated via UV absorption measurements at fixed wavelength. A UV/VIS-Spectrometer V-550 from Jasco (Groß-Umstadt, Germany) was used in this purpose. To 5 mg of dry loaded resin, $2 \mathrm{ml}$ of DMF and $40 \mu \mathrm{L}$ of DBU [1,8-diazabicyclo (5.4.0) undec-7-en] were added and was shaken for $2 \mathrm{~h}$ in a shaker at room temperature while the loaded resin beads were always immersed into the deprotection solution. Acetonitrile was added to make it $10 \mathrm{ml}$ and then the mixture was further diluted with acetonitrile to $25 \mathrm{ml}$. Another same system was prepared but without the loaded resin to use it for blank measurement. The final diluted solution was transferred to a UV precision cuvette $(1 \mathrm{~cm} \times 1 \mathrm{~cm})$. The UV-absorption of the cleaved Fmocdibenzofulvene species was detected at $304 \mathrm{~nm}$ assuming $\boldsymbol{\varepsilon}_{304}=7624 \mathrm{~L} \mathrm{~mol}^{-1} \mathrm{~cm}^{-1}[2]$. The actual loading of the first amino acid to the resin was calculated based on Lambert-Beer's law:

$$
\rho(\text { resin })\left[\frac{m m o l}{g}\right]=\frac{1}{m(\text { resin })[g]} \times\left(\frac{A[A U] \cdot V[L] \cdot f}{\varepsilon\left[M^{2} \mathrm{~cm}^{2}\right] \cdot d[\mathrm{~cm}]}\right) \times 10^{3}
$$

Where, $\boldsymbol{\rho}(\boldsymbol{r e s i n})$ is the actual loading density of the resin

$\boldsymbol{A}$ is the absorption

$\boldsymbol{V}$ is the volume of the flask (in this case $1 \mathrm{ml}$ )

$f$ is the thinning factor

$\varepsilon$ is the absorption coefficient of cleaved Fmoc-dibenzofulvene species 
$\boldsymbol{m}$ is the mass of the resin

$\boldsymbol{d}$ is the cuvette path length

\subsubsection{Circular dichroism spectroscopy (CD)}

CD spectroscopy measurements were carried out using a Jasco-1500 (Groß-Umstadt, Germany) spectropolarimeter equipped with a Julabo F250 (Seelbach, Germany) temperature controlling unit. The sample cells were flushed with nitrogen gas approximately 30 minutes prior to beginning of the measurements. The sample measurements were done in a quartz glass precision cuvette Suprasil ${ }^{\circledR}$ Hellma (type: 110-QS, $1.0 \mathrm{~mm}$ ). The spectra were recorded at $25^{0}$ and $50^{\circ} \mathrm{C}$ in a wavelength range of $250-185 \mathrm{~nm}$ with a data pitch of $1.0 \mathrm{~nm}, 1.0 \mathrm{~nm}$ bandwidth, using continuous mode, scanning time of $50 \mathrm{~nm} / \mathrm{min}$, a response time of $1.0 \mathrm{sec}$ and an average of 30 spectra. The CD-measurements were performed either in organic solvents like TFE or in vesicle suspensions containing P:L ration as 1:50 in TRIS buffer (10 $\mathrm{mMolL}^{-1}$ TRIS / $100 \mathrm{mMolLit}^{-}$ ${ }^{1} \mathrm{NaCl}, \mathrm{pH}=7.4$ ) The peptide concentrations were kept constant at $30 \mu \mathrm{M}$. The samples were background corrected against a vesicle suspension without incorporated peptide as blank and the spectra were smoothed using Savitzky-Golay function. The spectra were converted into molar ellipticity $\theta\left(\mathrm{deg} . \mathrm{cm}^{2} . \mathrm{dmol}^{-1}\right)$ by Greenfield's equation taking the concentration into account:[3]

$$
\theta\left[\mathrm{deg} \cdot \mathrm{cm}^{2} \cdot \mathrm{dmol}{ }^{-1}\right]=\frac{C D}{\mathrm{c} \cdot \mathrm{d} \cdot N_{A A}}
$$

$\begin{array}{ll}\text { CD: } & \text { ellipticity [mdeg] } \\ \boldsymbol{c}: & \text { concentration of the peptide }\left[\mathrm{mol}^{-1} \mathrm{~L}^{-1}\right] \\ \boldsymbol{d}: \quad \text { path length of the cuvette }[\mathrm{mm}] \\ \boldsymbol{N}_{\boldsymbol{A A}:} \quad \text { number of residues }\end{array}$

\subsubsection{Gas chromatography (GC)}

Gas chromatography was performed on an Agilent Technologies (Waldbronn, Germany) chromatograph 7890A GC System (type: Supelcowax 10 Fused Silica Capillary Column; 30 m x 
$0.32 \mathrm{~mm} \times 0.25 \mu \mathrm{m})$. GC calibrations were carried out with authentic samples and dodecane was used as an internal standard.

\subsubsection{Gas chromatography Mass Spectroscopy (GC-MS)}

GC-MS spectra were recorded on ThermoFinnigan (Schwerte, Germany) Spectrometers TRACE (Varian GC Capillary Column; wcot fused silica coated CP-SIL 8CB for amines; $30 \mathrm{~m} \mathrm{x} 0.32 \mathrm{~mm}$ x $0.25 \mu \mathrm{m}$ ) and DSQ (Varian FactorFour Capillary Column; VF-5ms $30 \mathrm{~m}$ x $0.32 \mathrm{~mm}$ x $0.25 \mu \mathrm{m}$ ) equipped with a TRIPLUS RSH autosampler.

\subsection{General Procedures (G.P)}

\subsubsection{General procedure to synthesize $\boldsymbol{\beta}^{3}$-D-homo-amino acids}

\subsubsection{G.P -1: Synthesis of diazoketone from corresponding $\alpha$-amino acids}

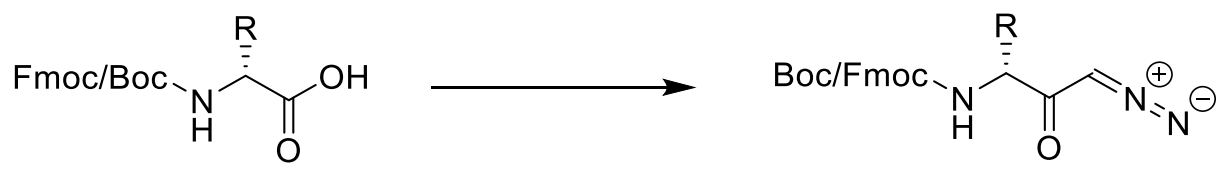

Based on the method developed by Guichard et.al, the N-Fmoc/Boc protected $\alpha$-amino acid (1.0 eq) was dissolved in dry THF under argon atmosphere with continuous stirring and was cooled to $-25^{\circ} \mathrm{C}$ followed by the addition of triethylamnine $(1.10 \mathrm{eq})$ and isobutylchloroformate $(1.10$ eq). After stirring the reaction mixture at $-25^{\circ} \mathrm{C}$ for 45 minutes, diazomethane (0.6.0.7 $\left.\mathrm{M}, 2.0 \mathrm{eq}\right)$ was carefully added to the reaction vessel under complete exclusion of light and the mixture was stirred at $0^{\circ} \mathrm{C}$ for 30 minutes followed by stirring at room temperature for $4.5 \mathrm{hrs}$. The reaction progress was monitored by TLC in the interim period. After the reaction was completed with almost no starting material left, glacial acetic acid (2.0 eq) was added to quench the excess diazomethane. The reaction mixture was further washed with aqueous $6 \% \mathrm{NaHCO}_{3}$ solution (approx. $3 \times 80 \mathrm{~mL}$ ) and extracted with EtOAc $(3 \times 100 \mathrm{~mL})$. The combined organic phases were washed with saturated aqueous $\mathrm{NH}_{4} \mathrm{Cl}(3 \times 60 \mathrm{~mL}$ ), saturated aqueous brine solution (aq. Nacl, 3 $\times 60 \mathrm{~mL}$ ) and dried over $\mathrm{Na}_{2} \mathrm{SO}_{4}$. The excess solvent was removed under educed pressure and 
the resulting product (yellow oil/solid) was used in the subsequent step without further purification.

\subsubsection{G.P -2: Synthesis of $\beta^{3}$-D-amino acid from the corresponding diazo-ketone intermediate}<smiles></smiles>

According to the methodology developed by Guichard et al., the diazo-ketone intermediate (1.00 eq.) was dissolved in a solvent mixture composed of THF/water $(9: 1, v / v)$ and silver benzoate (0.10 eq.) was added under complete exclusion of light. The reaction vessel is cooled in an icebath while adding the silver benzoate and afterwards placed in an ultrasonication water-bath for the reaction to continue for $2 \mathrm{H}$ under sonication. After completion of the reaction, the mixture was diluted with water (approx. $50 \mathrm{~mL}$ ); pH was adjusted to 2-3 by adding 1.0 M HCL (aq.) and extracted with EtOAc $(3 \times 80 \mathrm{~mL})$. The combined organic phases were dried over $\mathrm{Na}_{2} \mathrm{SO}_{4}$ and the solvent was removed under reduced pressure. The final rude product was purified either by flash column chromatography (Table 1) or by precipitation (crude product taken in DCM and was poured drop-wise into ice-cold pentane. The precipitate was filtered off, washed further with cold pentane for two times and dried overnight under reduced pressure).

\begin{tabular}{|c|c|c|}
\hline $\boldsymbol{\beta}^{3}$-D-Homo-Amino Acids & Yield in \% & Method of Purification \\
\hline Fmoc- $\beta^{3}$-D-Lys(Boc)-OH & 83 & $\begin{array}{c}\text { Precipitation in cold pentane } \\
\text { Pentane : DCM (12:1) }\end{array}$ \\
\hline Fmoc- $\beta^{3}$-D-Val-OH & 88 & $\begin{array}{c}\text { Precipitation in cold pentane } \\
\text { Pentane : DCM (12:1) }\end{array}$ \\
\hline Fmoc- $\beta^{3}$-D-Leu-OH & 73 & $\begin{array}{c}\text { Flash column chromatography } \\
\text { DCM : MeOH (10:1) }\end{array}$ \\
\hline
\end{tabular}




\begin{tabular}{|c|c|c|}
\hline Fmoc- $\beta^{3}$-D-Ala-OH & 90 & $\begin{array}{l}\text { Precipitation in cold pentane } \\
\text { Pentane : DCM (12:1) }\end{array}$ \\
\hline Fmoc- $\beta^{3}$-D-Trp(Boc)-OH & 72 & $\begin{array}{c}\text { Flash column chromatography } \\
\text { DCM : MeOH }(24: 1)\end{array}$ \\
\hline Boc- $\beta^{3}$-D-Lys(Fmoc)-OH & 70 & $\begin{array}{c}\text { Flash column chromatography } \\
\text { DCM : } \mathrm{MeOH}(18: 1)\end{array}$ \\
\hline Boc- $\beta^{3}$-D-Val-OH & 85 & $\begin{array}{c}\text { Flash column chromatography } \\
\text { DCM : } \mathrm{MeOH}(12: 1)\end{array}$ \\
\hline Boc- $\beta^{3}$-D-Leu-OH & 64 & $\begin{array}{c}\text { Flash column chromatography } \\
\text { DCM : MeOH }(18: 1)\end{array}$ \\
\hline Boc- $\beta^{3}$-D-Ala-OH & 89 & $\begin{array}{l}\text { Precipitation in cold pentane } \\
\text { Pentane : DCM }(12: 1)\end{array}$ \\
\hline Boc- $\beta^{3}$-D-Trp(For)-OH & 63 & $\begin{array}{c}\text { Flash column chromatography } \\
\text { DCM : } \mathrm{MeOH}(24: 1)\end{array}$ \\
\hline
\end{tabular}

Table 8.1. Methods to purify different $\beta^{3}$-D-homo-amino acid building blocks.

\subsubsection{General procedure to synthesize $\boldsymbol{\beta}$-peptides}

\subsubsection{G.P - 3: General procedure for Fmoc-deprotection in microwave assisted manual SPPS}

The resin was washed thoroughly with NMP or DMF (5 x $5 \mathrm{~mL}$ ) followed by addition of a cocktail of $20 \%$ piperidine in NMP. First deprotection was carried out in microwave with 30 $\mathrm{sec} / 25 \mathrm{~W} / 50^{\circ} \mathrm{c}$ condition. Subsequently the second deprotection was performed by adding a deprotection cocktail of DBU/piperidine/DMF (1:5:44) into the resin and carried out with 3 $\mathrm{min} / 35 \mathrm{~W} / 60^{\circ} \mathrm{C}$ condition in the microwave. The resin was washed with DMF (7 x $\left.5 \mathrm{~mL}\right)$, DCM $(4 \times 5 \mathrm{~mL})$ and NMP (4 $55 \mathrm{~mL})$. 


\subsubsection{G.P - 4: General procedure for Fmoc-deprotection in manual SPPS without microwave}

The resin was washed thoroughly with DMF (5 x $5 \mathrm{~mL}$ ) followed by addition of a cocktail of $20 \%$ piperidine in DMF and shaken in a shaker at room temperature for 15-20 min. The second Fmocdeprotection was carried out by the subsequent addition of a cocktail of $20 \%$ piperidine and $2 \%$ DBU in DMF and shaken at room temperature for 5-10 min. The resin was then washed thoroughly with solution of $\{$ DCM/MeOH/DIPEA (17/32/1)\} (5 x $5 \mathrm{~mL})$, DMF (5 x $5 \mathrm{~mL})$, DCM (5 x $5 \mathrm{~mL}$ ).

\subsubsection{G.P - 5: General procedure to load first amino acid ( $\beta^{3}$-D-homo-amino acid) to rink- amide MBHA or NovaPEG rink-amide low loaded resins}

Rink amide MBHA resin with a loading capacity of $0.57 \mathrm{mmol} / \mathrm{g}$ (used for $\beta^{3}$-D-Val peptide of 14 helical secondary structure) or rink amide NovaPEG resin with a loading capacity of 0.18 $\mathrm{mmol} / \mathrm{g}$ (used for $\beta^{3}$-D-Leu peptide of 12-helical secondary structure) (0.1 mmol, 1 equiv) was placed in a $10 \mathrm{~mL}$ PE-frit equipped Becton-Dickinson(BD) discardit II syringe (Heidelberg, Germany) and was swollen with NMP or DMF (6 mL) for $60 \mathrm{~min}$. To the rink-amide MBHA resin was then added Fmoc-deprotection mixture $(5 \mathrm{~mL})(20 \%$ piperidine in NMP or DBU/piperidine/DMF (1:5:44) + LiCl 0.3 M) and the deprotection was carried out in microwave assistance in a CEM Discover apparatus. The Fmoc-deprotection was carried out in 2 steps; at first for $30 \mathrm{sec} / 25 \mathrm{~W} / 50^{\circ} \mathrm{c}$ and then for $3 \mathrm{~min} / 35 \mathrm{~W} / 60^{\circ} \mathrm{C}$ in the microwave. No deportation was required for rink amide NovaPEG resin. After the last step, the resin was washed successively with DMF (7 x $5 \mathrm{~mL})$, DCM (4 x $5 \mathrm{~mL}$ ) and NMP (4 x $5 \mathrm{~mL})$. Afterwards the coupling of the first amino acid was performed by dissolving the first Fmoc- $\beta^{3}$-D-homo amino acid (5 eq.) and anhydrous HOBt (5 eq.) in $1.8 \mathrm{~mL} \mathrm{NMP}$ and to the solution was added DIC (5 eq.) The mixture was vortexed for $5 \mathrm{sec}$ and transferred to the resin and the coupling reaction took place with microwave assistance $\left(15 \mathrm{~min} / 35 \mathrm{~W} / 65^{\circ} \mathrm{C}\right)$. Re-coupling of the first amino acid was carried out in microwave assistance ( $20 \mathrm{~min} / 25 \mathrm{~W} / 50^{\circ} \mathrm{C}$ ) using the amino acid (3 eq.), anhydrous $\mathrm{HOBt}$ ( 3 eq.) and DIC (3 eq.) in $1.5 \mathrm{~mL}$ NMP. After each coupling step, the resin was washed successively with DMF (7 x $5 \mathrm{~mL}), \mathrm{MeOH}(4 \times 5 \mathrm{~mL})$ and DCM (4 x $5 \mathrm{~mL}$ ). The resin was dried under vacuum overnight. The estimation of the exact loading efficacy was carried out by UV-spectroscopic method as described in $\mathbf{6} . \mathbf{2} .4$ section. 


\subsubsection{G.P - 6: General procedure to cap free amino groups in microwave-assisted SPPS}

For capping of free amino groups, acetic anhydride (10 eq.), DIPEA (15 eq.) and DMAP (0.1 eq.) were dissolved in $1.5 \mathrm{~mL}$ DMF in a $2.0 \mathrm{~mL}$ - eppendorf vial and the mixture was purged with Arstream for 3 min followed by transferring to the resin. The resulting mixture was shaken mechanically for $1.5 \mathrm{~h}$ at RT. Then the resin was washed successively with a solution of \{DCM/MeOH/DIPEA (17/32/1)\} (5 x $5 \mathrm{~mL})$, DMF (5 x $5 \mathrm{~mL}$ ), DCM (5 x $5 \mathrm{~mL}$ ) and subsequently was dried and stored under vacuum.

\subsubsection{G.P - 7: General procedure to load first amino acid ( $B^{3}$-D-homo-amino acid or $B^{2}-D$ - homo-amino acid) to 2-Chlorotrityl chloride resin ${ }^{[8]}$}

The Fmoc- $\beta$-D-amino acid ( 3 eq. to the resin) and DIPEA (6 eq. to the resin) were dissolved in DCM $(10 \mathrm{~mL} / \mathrm{g}$ resin). The dry resin was taken into a BD-syringe equipped with a PE filter from Becton-Dickinson and pre-swollen in DCM for $1 \mathrm{hr}$. After the resin was properly swollen, the solution containing the amino acid was added and the resin was shaken at room temperature for $1 \mathrm{hr}$. The resin was thoroughly with a solution of $\{\mathrm{DCM} / \mathrm{MeOH} / \mathrm{DIPEA}(17 / 32 / 1)\}(5 \times 3 \mathrm{~mL})$, DMF (5 $33 \mathrm{~mL})$, DCM ( $5 \times 3 \mathrm{~mL})$. Deprotection of the Fmoc-group of the first loaded amino acid was carried out by applying a solution of $20 \%$ piperidine in DMF ( 2 x $30 \mathrm{~min})$. After the deprotection, the resin was again washed with a solution of $\{\mathrm{DCM} / \mathrm{MeOH} / \mathrm{DIPEA}(17 / 32 / 1)\}(5 \mathrm{x}$ $3 \mathrm{~mL}$ ), DMF (5 x $3 \mathrm{~mL}$ ), DCM (5 x $3 \mathrm{~mL}$ ) subsequently.

\subsubsection{G.P - 8: General procedure to coupling in microwave assisted manual SPPS ${ }^{[9,10]}$}

\section{For rink amide MBHA resin:}

Microwave-assisted manual SPPS was carried out in a PE-frit equipped Becton-Dickinson (BD) discardit II syringe (Heidelberg, Germany) with a Discover microwave (MW) reaction cavity (CEM, Kamp-Lintfort, Germany). The pre-loaded resin was swollen in NMP for $2 \mathrm{~h}$ in a shaker followed by addition of the deprotection cocktail to deprotect the Fmoc-group of the pre-loaded amino acid and the two-step deprotection was carried out as described in G.P - 3. After the post 
deprotection washing of the resin with DMF $(7 \times 5 \mathrm{~mL})$, DCM (4 x $5 \mathrm{~mL})$ and NMP (4 x $5 \mathrm{~mL})$, the peptide coupling was performed twice with Fmoc-deprotected amino acid (5 eq., 3.0 eq.) HATU (4.5 eq., 2.7 eq.), HOAt (5.0 eq., 3.0 eq.) and DIPEA (10.0 eq. x 2) dissolved in 2 mL NMP. The microwave conditions for first and second couplings were $\left(20 \mathrm{~min} / 50^{\circ} \mathrm{C} / 25 \mathrm{~W}\right)$ and $(15 \mathrm{~min} /$ $60^{\circ} \mathrm{C} / 35 \mathrm{~W}$ ), respectively. After the coupling the resin was washed with DMF (7 x $\left.5 \mathrm{~mL}\right)$, DCM (4 x $5 \mathrm{~mL}$ ) and NMP (4 x $5 \mathrm{~mL}$ ) and a capping of the free amino groups was performed as described in G.P - 6. The Fmoc-deprotection of the coupled amino acid was done in correspondance with the method described in G.P - 3.

\section{For rink amide NovaPEG LL resin:}

This particular resin with low loading capacity was used to synthesize very hydrophobic peptide composed of $\beta^{3}$-D-Leu amino acids. To remove the Fmoc-group a deprotection cocktail of 10\% DBU, $20 \%$ piperidine in NMP was used twice in $3 \mathrm{~min} / 60^{\circ} \mathrm{C} / 25 \mathrm{~W}$ microwave conditions following G.P - 3. Next, the resin was thoroughly washed with DMF (7 x $5 \mathrm{~mL}$ ), DCM (4 x $5 \mathrm{~mL}$ ) and NMP ( 4 × $5 \mathrm{~mL}$ ). The coupling was performed twice with the desired amino acid ( 5 eq. 5 eq.), HATU (4.5 eq. 4.5 eq.), HOAt (5.0 eq. 5.0 eq.), anhydrous LiCl (0.3 M) and DIPEA (10.0 eq. 10.0 eq.) dissolved in $3 \mathrm{~mL}$ NMP with microwave conditions $\left(20 \mathrm{~min} / 65^{\circ} \mathrm{C} / 25 \mathrm{~W}\right)$ and $(15 \mathrm{~min} /$ $75^{\circ} \mathrm{C} / 35 \mathrm{~W}$ ), respectively. The resin was again washed with DMF (7 x $5 \mathrm{~mL}$ ), DCM (4 x $\left.5 \mathrm{~mL}\right)$ and NMP ( 4 x $5 \mathrm{~mL}$ ) and a capping of the free amino groups was performed as described in G.P - 6 .

\subsubsection{G.P - 9: General procedure to coupling in non- microwave assisted manual SPPS in room temperature ${ }^{[8]}$}

Manual SPPS was carried out in a PE-frit equipped Becton-Dickinson (BD) discardit II syringe (Heidelberg, Germany). The dry preloaded resin was swollen in DCM for $1 \mathrm{~h}$ and the Fmocdeprotection was carried out following G.P - 4. The resin was washed with a solution of \{DCM/MeOH/DIPEA (17/32/1)\} (5 x $3 \mathrm{~mL})$, DMF (5 x $3 \mathrm{~mL})$, DCM (5 x $3 \mathrm{~mL})$. The desired $\beta^{3}$ or $\beta^{2}$-D-amino acids (2.0 eq. to the resin) followed by DMT/NMM/TsO- (coupling reagent, 2 eq. to the resin) and DIPEA (4 eq. to the resin) were dissolved in $3 \mathrm{~mL}$ of DCM and added to the resin. The coupling reaction was performed for $2 \mathrm{~h}$ at room temperature in a shaker. The resin was washed again with $\{$ DCM/MeOH/DIPEA (17/32/1)\} (5 x $3 \mathrm{~mL})$, DMF (5 x $3 \mathrm{~mL}), \mathrm{DCM}(5 \times 3 \mathrm{~mL})$ and capping of the free amino groups was carried out following G.P - 6. 


\subsubsection{G.P - 10: Kaiser Test: A general method for qualitative detection of coupling}

Kaiser test is a simple qualitative test to verify whether the amino acids were efficiently coupled to the resin via determination of the free amino groups. It was carried out by putting a few small beads of resin into a small test tube and adding two-three drops of the following Kaiser Test solutions into it:

Solution 1: Phenol in ethanol $(4 \mathrm{~g} / \mathrm{mL})$

Solution 2: Ninhydrin in ethanol $(0.05 \mathrm{~g} / \mathrm{mL})$

Solution 3: Aqueous potassium cyanide (1 mM, $2 \mathrm{~mL})$ in pyridine (98 $\mathrm{mL})$

After adding the Kaiser Test solutions to the resin beads, the suspension was heated for 5 minutes at $100^{\circ} \mathrm{C}$. If the solution turned blue in colour, it suggests a positive test implying presence of free amino groups in the resin. This signifies that the coupling is incomplete and non-efficient. On the other hand, if the solutions becomes yellow in colour, that suggests a negative Kaiser Test implying absence of free amino groups. This qualitatively estimates that the coupling has been complete and efficient.

\subsubsection{G.P - 11: General procedure for the attachment of fluorophores to peptide}

The resin-bound Fmoc-deprotected peptide (0.0005 mol, 1 eq.) was swollen in DCM for 2 hr. A solution of 5(6)-Carboxyfluorescein ( $0.0025 \mathrm{~mol}, 5.0$ eq.) in DMF (1 mL) was prepared and was activated with PyBOP ${ }^{\circledR}$ (0.0023 mol, 4.7 eq.) and DIPEA (0.0049 mol, 9.8 eq.). The reaction was carried out overnight at $65^{\circ} \mathrm{C}$ inside a temperature controlled Thermo-Shaker under complete exclusion of light. Next day, a solution of piperidine in DMF (20:80) was added to the resin in order to remove phenol esters produced and the resin was shaken for $30 \mathrm{~min}$ at room temperature. The resin was washed with $\mathrm{DMF}, \mathrm{MeOH}, \mathrm{DCM}$ and dried under reduced pressure.

\subsubsection{G.P - 12: General procedure to cleave the peptide from the resin}

The peptides were cleaved from the resin using a cleavage cocktail of HFIP/DCM (3:7) (approx. 5 $\mathrm{mL} / 0.1 \mathrm{~g}$ resin). The cleavage cocktail was added to the dried resin and shaken at room temperature for $1-1.5 \mathrm{hr}$. The resin was filtered off, and washed with DCM (3 x $3 \mathrm{~mL})$ followed 
by the evaporation of the filtrate under pure $\mathrm{N}_{2}$-stream. To the residue, was added cold $\left(-21^{\circ} \mathrm{C}\right)$ $\mathrm{Et}_{2} \mathrm{O}$ to precipitate the peptide. The mixture was centrifuged at $9000 \mathrm{rpm}$ for $20 \mathrm{~min}$ and the supernatant was again evaporated under $\mathrm{N}_{2}$-stream. The crude peptide was further purified by R.P-HPLC and collected fractions were lyophilized to yield the pure desired peptide. The entire process was carried out very cautiously in absence of light by covering the syringes and vials with aluminum-foil.

\subsubsection{G.P - 13: General procedure for $\mathrm{N}$-(Boc)-deprotection in solution phase}

The N(Boc)-protected amino acid was dissolved in TFA ( $5 \mathrm{~mL} / 1 \mathrm{mmol})$ at $0^{\circ} \mathrm{C}$. The reaction mixture was stirred for $45 \mathrm{~min}$ at $0^{\circ} \mathrm{C}$ and for $1.5 \mathrm{~h}$ at room teperature. Afterwards the excess solvent was evaporated under reduced pressure while a co-evaporation with toluene ( $2 \mathrm{x}$ ) was performed. Then the residue was dried under high vacuum for overnight, which yielded the crude TFA salt of the desired peptide. This was used in the next coupling without further purification.

\subsubsection{G.P - 14: General procedure for acid fluoride coupling in solution phase}

The N(Boc)-deprotected amino acid (1 eq.) was dissolved in DMF (20 mL/1 mmol) under sonication and NMM (4 eq.) as well as the N(Boc)-protected acid fluoride (2 eq.) was added to it. The reaction was carried out at room temperature to high temperature (between $90^{\circ}-120^{\circ} \mathrm{C}$ ) for $3 \mathrm{~h}$ to $5 \mathrm{~min}$, respectively. On completion, the mixture was diluted with dichloromethane and washed with aqueous $\mathrm{KH}_{2} \mathrm{PO}_{4}$ solution $\left(1 \mathrm{M}\right.$ ), aqueous $\mathrm{K}_{2} \mathrm{HPO}_{4}$ solution $(1 \mathrm{M})$, and brine. The combined organic fractions were dried over $\mathrm{Na}_{2} \mathrm{SO} 4$ and the solvent was removed under reduced pressure. The crude peptide was purified by fluorous solid phase extraction (FSPE) prior to the beginning of the next coupling cycle.

\subsubsection{G.P - 15: General procedure for fluorous solid phase extraction (FSPE) purification [14]}



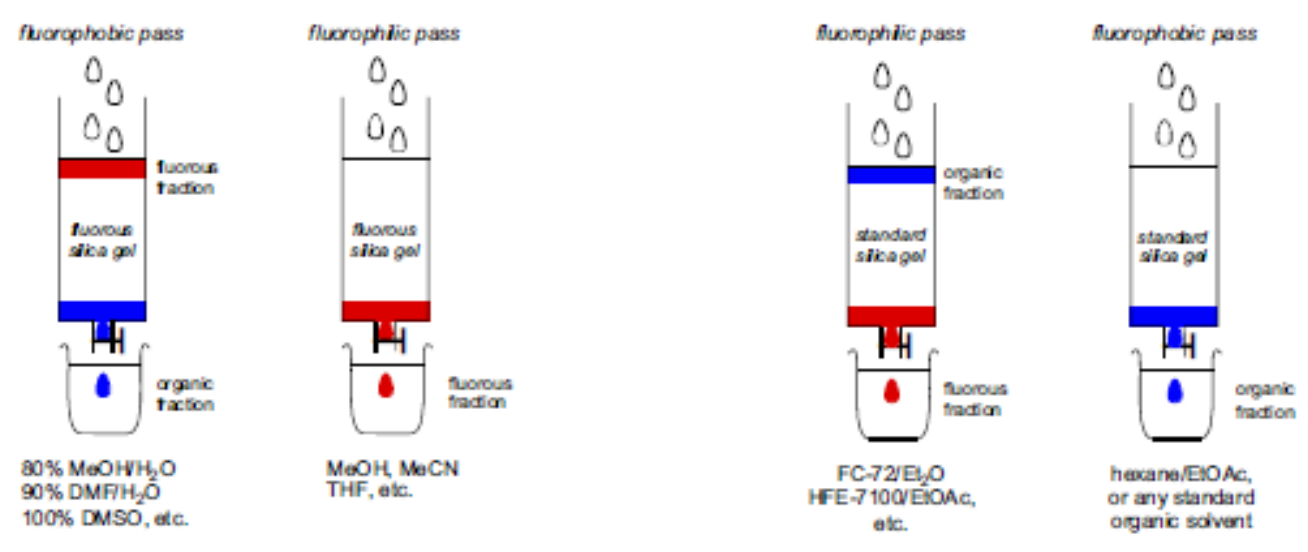

Figure 8.2: Taken from W. Zhang et. al. Tetrahedron 62 (2006) 11837-11865[15]

The glass cartridge $(5 \mathrm{~mL})$ was packed with the fluorous silica gel (Fluoroflash ${ }^{\circledR}$ Silica Gel $40 \mu \mathrm{m}$ ) by making a slurry in DMSO. Then the packed column was washed with $2 \mathrm{~mL}$ of DMF under vacuum. Next, a pre-conditioning of the column was required and 6-8 $\mathrm{mL}$ of $100 \%$ DMSO was passed through it and discarded. Thereafter the heavy fluorous-tagged crude sample peptide (100-200 mg) was dissolved in 0.4 mL DMF and loaded onto the column using vacuum to ensure that the sample was thoroughly adsorbed onto the packed column. Here it was made sure that the weight of the sample loaded must be less than $30 \%$ of the weight of the fluorous silica gel to prevent 'breakthrough' issue, where a small amount of the fluorous tagged sample gets eluted right in the beginning while the rest of the sample gets retained. Next, the fluorophobic wash was carried out to elute the non-fluorous fractions. The fluorophobic wash was performed with 6-8 $\mathrm{mL}$ of $100 \%$ DMSO or $80: 20 \mathrm{MeOH} /$ water. Now after the non-fluorous fractions were collected, a fluorophilic wash was carried out with 8-9 mL of fluorophilic solvents like $100 \% \mathrm{MeOH}$ or $100 \%$ acetone to collect the pure heavy-fluorous-tagged fractions of the desired peptide. Thus, the fluorous fractions were concentrated under pure $\mathrm{N}_{2}$-stream to get the pure fluorous-tagged peptide that was used in the next acid fluoride coupling cycle in solution phase.

\subsubsection{General procedure to prepare peptide/lipid vesicles}

\subsubsection{G.P - 16: General procedure to generate multilamellar vesicles (MLVs)}

The lipid systems, such as, 1-palmitoyl-2-oleoyl-sn-glycero-3-phosphocholine (POPC), 1,2dilauroyl-sn-glycero-3-phosphocholine (DLPC), 1,2-dimyristoyl-sn-glycero-3-phosphocholine 
(DMPC), 1,2-dierucoyl-sn-glycero-3-phosphocholine \{22:1 (cis) PC $\}$ and 1,2-dinervonoyl-snglycero-3-phosphocholine, were dissolved either in pure $\mathrm{CHCl}_{3}$ or in $\mathrm{CHCl}_{3}$ with different percentages of EtOH. The peptides were dissolved in TFE and thereafter the lipid and peptide solutions were mixed together attaining different peptide-to-lipid ratios (P:L). This peptide/lipid mixture was evaporated under pure nitrogen stream at temperature above the specific transition temperatures $\left(T_{m}\right)$ of the lipids $\left(\boldsymbol{T}_{\boldsymbol{m}}{ }^{P O P C}=-2^{\circ} \mathrm{C} ; \boldsymbol{T}_{\boldsymbol{m}}{ }^{D L P C}=-1^{0} \mathrm{C} ; \boldsymbol{T}_{\boldsymbol{m}}{ }^{D M P C}=24^{0} \mathrm{C} ; \boldsymbol{T}_{\boldsymbol{m}^{22: 1}}\right.$ (cis)PC $=13.2^{\circ} \mathrm{C} ; \boldsymbol{T}_{m}{ }^{24: 1}\left(\right.$ cis)PC $=2^{\circ} \mathrm{C}$ ).[11, 12] $\mathrm{TFE}$ was added to the dried peptide-lipid film in order to support the peptide to form secondary structure and was again evaporated under similar condition. The thin peptide-lipid film on the inner wall of the glass vial was further dried overnight in high vacuum oven at temperature higher than that of the respective transition temperatures of the lipids to generate the transparent peptide-lipid films. The latter was rehydrated with TRIS $^{\circledR}$ buffer solution (100 mM NaCl, $10 \mathrm{mM}$ TRIS $^{\circledR}, \mathrm{pH}$ 7.4) and incubated for 2 $\mathrm{h}$ at temperature above the transition temperature. The suspension was vertexed a few times for 30 seconds with subsequent incubation for 5 min (5 cycles). The peptide-lipid multilamellar vesicle suspension was prepared and stored at temperature higher than that of the transition temperatures for respective lipids.

\subsubsection{G.P - 17: General procedure to generate large unilamellar vesicles (LUVs) [13]}

The suspension of multilamellar vesicles prepared by G.P - 13 was extruded 31 times through a polycarbonate membrane (100 nm nominal pore size) with an Avesatin Liposofast mini extruder (Ottawa, Canada) to generate clear large unilamellar vesicle (LUV) suspension containing the desired peptide in specific $\mathrm{P} / \mathrm{L}$ ratio.

\subsubsection{General procedures for in vitro studies of $\boldsymbol{\beta}$-peptides as potent cell penetrating peptide candidates on human malignant cell lines}

\subsubsection{G.P - 18: General procedure to evaluate cytotoxicity of different $\beta$-peptides in vitro on human lung carcinoma cell line A549 in a colonogenic assay}


The adherent human bronchial adenocarcinoma cells, A549 (ATCC CCL - 185), were cultured in DMEM (Lonza) supplemented with 10\% (v/v) fetal calf serum, $4 \mathrm{mmol} \mathrm{L-glutamine} \mathrm{(Biochrom)}$ at $370 \mathrm{C}$ in $7.5 \%$ carbon dioxide environment. Tissue culture dishes (6-well multiplates, TPP) were seeded with $10^{2}-10^{4}$ cells per well. The cells were allowed to attach for $24 \mathrm{~h}$. Then the medium was replaced with a serum free environment (Ultra Culture, Lonza) with freshly prepared compounds ( $\beta$-peptides, pre-dissolved in DMSO, 1\% final concentration in serum free medium) for $24 \mathrm{~h}$ at different concentrations. Afterwards, the drug ( $\beta$-peptides) treated cells were cultured in normal culture medium for 7 days to form colonies. They were fixed and stained with methylene blue (Merck) and cell counting was carried out. Plating efficiencies of the drug ( $\beta$-peptides) treated cells, relative to non-treated cells, were calculated and plotted.

\subsubsection{G.P - 19: General procedure to evaluate cytotoxicity of different $\beta$-peptides in vitro on human lung carcinoma cell line A549 and human mammary gland adenocarcinoma cell line MCF-7 (ATCC HTB-22) in a MTS assay}

The cell lines used in MTS assay were the adherent human bronchial adenocarcinoma cell line A 549 (ATCC CCL-185) and human mammary gland adenocarcinoma cell line MCF-7 (ATCC HTB22). A549 cells were cultured in DMEM (Lonza) supplemented with $10 \%$ (v/) fetal calf serum, 4 mmol L-glutamine (Biochrom) at $37^{\circ} \mathrm{C}$ in a humidified, $7.5 \%$ carbon dioxide atmosphere whereas the MCF-7 cells were cultured in RPMI 1640 (Lonza) supplemented with 10\% (v/v) fetal calf serum, $2 \mathrm{mmol}$ L-glutamine (Biochrom) at $37^{\circ} \mathrm{C}$ in a humidified, $5 \%$ carbon dioxide atmosphere. The tissue culture dishes (96-well multiplates, TPP) were seeded with 12000 cells per well in $50 \mu \mathrm{L}$ of serum-free medium (Ultra Culture, Lonza) with freshly prepared compounds ( $\beta$-peptides, pre-dissolved in DMSO, 1\% final concentration in medium) for $48 \mathrm{~h}$ at different concentrations at $37^{\circ} \mathrm{C}$ in a humidified, $5 \%$ carbon dioxide atmosphere. $20 \mu \mathrm{L}$ per well of CellTiter ${ }^{\circledR} 96 \mathrm{AQ}_{\text {ueous }}$ One Solution Reagent (Promega) were added and incubated for $2 \mathrm{~h}$ at $37^{\circ} \mathrm{C}$ in a humidified, 5\% carbon dioxide atmosphere. The absorbance were recorded at $490 \mathrm{~nm}$ (and $650 \mathrm{~nm}$ as a reference) using a 96-well plate reader. Plating efficiencies of drug ( $\beta$-peptides)treated cells, relative to untreated cells, were calculated and plotted. 


\subsubsection{G.P - 20: General procedure fluorescence-activated cell sorting (FACS) flow cytometry technique for quantitative estimation of cellular uptakes using human bronchial adenocarcinoma cell line (A549)}

The cell lines used were the adherent human bronchial adenocarcinoma cell line A549 (ATCC CCL-185). The cell lines were cultured in DMEM (Lonza) supplemented with $10 \%$ (v/v) fetal calf serum, $4 \mathrm{mmol}$ L-Glutamine (Biochrom) at $37^{\circ} \mathrm{C}$ in a humidified, $7.5 \% \mathrm{CO}_{2}$ atmosphere. Tissue culture disches (6-well multiplates, TPP) were seeded with 125000-150000 cells per well. Cells were allowed to attach for 48 hours, hen the wells were washed once with serum-free medium (Ultra Culture, Lonza). Then the freshly prepared compounds (15 $\mu \mathrm{M}$ pre-solved in DMSO, 1\% final DMSO concentration in serum-free medium) were added for 1 hour at $37^{\circ} \mathrm{C}$ in a humidified, $7.5 \% \mathrm{CO}_{2}$ atmosphere. Subsequently, the samples were washed twice in growth media and detached from the multiplate using $1 \mathrm{~mL}$ Trypsin for $2-3 \mathrm{~min}$ at $37^{\circ} \mathrm{C}$ in a humidified, $7.5 \% \mathrm{CO}_{2}$ atmosphere. Trypsination was inhibited using a 1:1 mixture of growth medium and fetal calf serum. For washing, cells were centrifuged at $1200 \mathrm{rpm}$ for $3 \mathrm{~min}$. and resuspended in growth medium. This washing step was repeated twice, resuspending cells in PBS both times. Cell counting was performed in a BD FACS Canto II using the BD FACS Diva Software. Gating for forward and side scatter was done with control (non-treated) cells, for FITC positive cells using HIV(1)-TAT treated cells as a control.

\subsubsection{G.P - 21: General procedure confocal laser scanning fluorescence microscopy to visualize the cellular uptakes of different target peptides on human bronchial adenocarcinoma cell line (A549)}

The cell lines used were the adherent human bronchial adenocarcinoma cell line A549 (ATCC CCL-185). The cell lines were cultured in DMEM (Lonza) supplemented with $10 \%(\mathrm{v} / \mathrm{v}$ ) fetal calf serum, $4 \mathrm{mmol}$ L-Glutamine (Biochrom) at $37^{\circ} \mathrm{C}$ in a humidified, $7.5 \% \mathrm{CO}_{2}$ atmosphere. Tissue culture disches (6-well multiplates, TPP) were seeded with 150000 cells per well. Cells were allowed to attach for 48 hours, hen the wells were washed once with serum-free medium (Ultra Culture, Lonza). Then the freshly prepared compounds (15 $\mu \mathrm{M}$ pre-solved in DMSO, 1\% final DMSO concentration in serum-free medium) were added for 1 hour at $37^{\circ} \mathrm{C}$ in a humidified, $7.5 \%$ $\mathrm{CO}_{2}$ atmosphere. Fluorescence of the samples were checked prior to washing by confocal microscopy. Confocal laser scanning fluorescence microscopy was performed using FluoView FV1200 (Olympus, Tokyo, Japan) equipped with a 60x oil immersion objective numerical 
aperture $(\mathrm{NA})=0.65-1.25\}$. Subsequently the samples were washed three times in growth media and imaged again using confocal laser scanning fluorescence microscopy. Image analysis was performed using Fiji [Schindelin, J.; Arganda-Carreras, I. \& Frise, E. et al. (2012) "Fiji: an open source platform for biological-image analysis", Nature methods 9(7): 676-682].

\subsection{Syntheses}

\subsubsection{Synthesis of amino acid derivatives}

\subsubsection{1 (S)-3-((((9H-fluoren-9-yl)methoxy)carbonyl)amino)-4methylpentanoic acid}

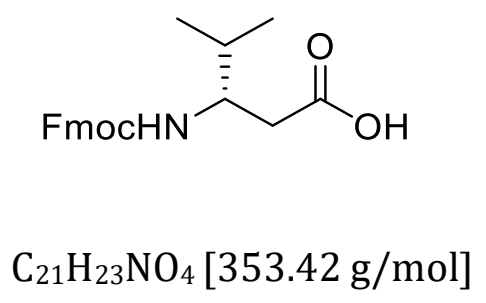

Synthetic Procedure: The N(Fmoc)- $\beta^{3}-\mathrm{D}-\mathrm{Val}-\mathrm{OH}$ was efficiently synthesized following the G.P 1 and G.P - 2 (see Table -1 for purification strategy and overall yield).

${ }^{1}$ H-NMR: $\left(300 \mathrm{MHz}, \mathrm{CDCl}_{3}\right.$ ): $\delta=0.95$ and $0.96\left(2 \mathrm{~d}, J=6.3,6.3 \mathrm{~Hz}, 6 \mathrm{H}, 2 \mathrm{CH}_{3}\right), 1.91$ (dqq, J=7.1 Hz, $1 \mathrm{H}$,

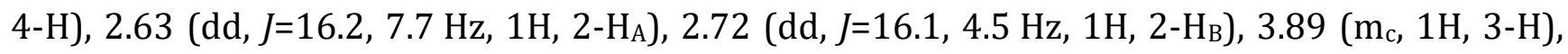
5.04-5.13 (m, 3H, $\left.\mathrm{OCH}_{2}, \mathrm{NH}\right), 7.31-7.38(\mathrm{~m}, 5 \mathrm{H}, \mathrm{Ph})$

ESI-MS: $(\mathrm{m} / \mathrm{z}): 354.4[\mathrm{M}+\mathrm{H}]^{+}, 376.3[\mathrm{M}+\mathrm{Na}]^{+}, 392.3[\mathrm{M}+\mathrm{K}]^{+}, 707.4[2 \mathrm{M}+\mathrm{H}]^{+}, 729.3[2 \mathrm{M}+\mathrm{Na}]^{+}$, $745.3[2 \mathrm{M}+\mathrm{K}]^{+}, 352.4[\mathrm{M}-\mathrm{H}]^{-}, 705.4[2 \mathrm{M}-\mathrm{H}]^{-}$.

HRMS (ESI): calc. for $\left[\mathrm{C}_{21} \mathrm{H}_{24} \mathrm{NO}_{4}\right]^{+}\left([\mathrm{M}+\mathrm{H}]^{+}\right): 354.4189$, found: 354.185 ; calc. for $\left[\mathrm{C}_{21} \mathrm{H}_{23} \mathrm{NO}_{4} \mathrm{Na}\right]^{+}$ $\left([\mathrm{M}+\mathrm{Na}]^{+}\right)$: 376.3309, found: 376.3315; calc. for $\left[\mathrm{C}_{21} \mathrm{H}_{22} \mathrm{NO}_{4}\right]^{-}\left([\mathrm{M}-\mathrm{H}]^{-}\right): 352.4011$, found: 352.4003 . 


\subsubsection{2 (3S)-3-(((9H-Fluoren-9-ylmethoxy)carbonyl)amino)-5-methylhexanoic acid}

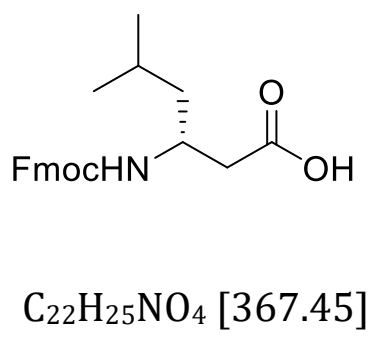

Synthetic Procedure: The N(Fmoc)- $\beta^{3}$-D-Leu-OH was efficiently synthesized following the G.P 1 and G.P - 2 (see Table -1 for purification strategy and overall yield).

${ }^{1} \mathrm{H}$ NMR (500.0 MHz, $\mathbf{C D C l}_{3}$ ): $\delta=0.90$ and 0.91 (2d, $J=6.2,6.2 \mathrm{~Hz}, 6 \mathrm{H}, 2 \mathrm{CH}_{3}$ ), 1.24-1.38, 1.51-1.64 ( $2 \mathrm{~m}, 3 \mathrm{H} ; 5-\mathrm{H}, 4-\mathrm{H}_{2}$ ), $2.61\left(\mathrm{dd}, J=16.5,5.5 \mathrm{~Hz}, 1 \mathrm{H}, 2-\mathrm{H}_{\mathrm{A}}\right.$ ), $2.72\left(\mathrm{dd}, J=16.5,5.1 \mathrm{~Hz}, 1 \mathrm{H}, 2-\mathrm{H}_{\mathrm{B}}\right), 4.07$

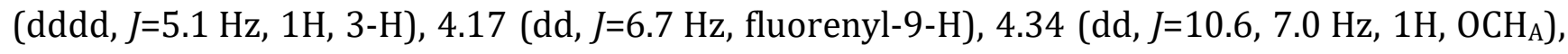
4.38 (dd, J=10.7, $\left.7.6 \mathrm{~Hz}, 1 \mathrm{H}, 0 \mathrm{CH}_{\mathrm{B}}\right), 5.05(\mathrm{~d}, J=9.1 \mathrm{~Hz}, 1 \mathrm{H}, \mathrm{NH}), 7.28$ (dd, J=7.8 Hz, 2H, fluorenyl-H), 7.37 (dd, J=7.3 Hz, 2H, fluorenyl-H), 7.55 (d, J=7.1 Hz, 2H, fluorenyl-H), 7.74 (d, $J=7.5 \mathrm{~Hz}, 2 \mathrm{H}$, fluorenyl-H).

ESI-MS: $(\mathrm{m} / \mathrm{z}): 368.4[\mathrm{M}+\mathrm{H}]^{+}, 390.5[\mathrm{M}+\mathrm{Na}]^{+}, 406.5[\mathrm{M}+\mathrm{K}]^{+}, 735.4[2 \mathrm{M}+\mathrm{H}]^{+}, 757.5[2 \mathrm{M}+\mathrm{Na}]^{+}$, $774.5[2 \mathrm{M}+\mathrm{K}]^{+}, 366.4[\mathrm{M}-\mathrm{H}]^{-}, 733.4[2 \mathrm{M}-\mathrm{H}]^{-}$.

HRMS (ESI): calc. for $\left[\mathrm{C}_{22} \mathrm{H}_{26} \mathrm{NO}_{4}\right]^{+}\left([\mathrm{M}+\mathrm{H}]^{+}\right)$: 368.4513, found: 368.4519; calc. for $\left[\mathrm{C}_{22} \mathrm{H}_{25} \mathrm{NO}_{4} \mathrm{Na}\right]^{+}\left([\mathrm{M}+\mathrm{Na}]^{+}\right): 390.5088$, found: 390.5079 ; calc. for $\left[\mathrm{C}_{22} \mathrm{H}_{24} \mathrm{NO}_{4}\right]^{-}\left([\mathrm{M}-\mathrm{H}]^{-}\right): 366.4121$, found: 366.4126

\subsubsection{3(S)-3-((((9H-fluoren-9myl)methoxy)carbonyl)amino)butanoic acid}<smiles>C[C@H](CC(=O)O)NC(F)F</smiles> 


$$
\mathrm{C}_{19} \mathrm{H}_{19} \mathrm{NO}_{4}[325.36 \mathrm{~g} / \mathrm{mol}]
$$

Synthetic Procedure: The N(Fmoc)- $\beta^{3}$-D-Ala-OH was efficiently synthesized following the G.P 1 and G.P - 2 (see Table -1 for purification strategy and overall yield).

${ }^{1} \mathrm{H}$ NMR (300.0 MHz, $\mathbf{C D C l}_{3}$ ): $\delta=1.30$ (d, J=6.7 Hz, 3H, 4- $\mathrm{H}_{3}$ ), 2.62 (br. s, 2H, 2- $\mathrm{H}_{2}$ ), 4.15 (br. s, 1H, 3-H), 5.10-5.24 (m, 3H, OCH $2, \mathrm{NH}), 7.32-7.42$ (m, 5H $\mathrm{H}_{\mathrm{Ar}}$ ), 10.5 (br. s, 1H, COOH).

ESI-MS: $(\mathrm{m} / \mathrm{z}): 326.3[\mathrm{M}+\mathrm{H}]^{+}, 348.3[\mathrm{M}+\mathrm{Na}]^{+}, 364.3[\mathrm{M}+\mathrm{K}]^{+}, 651.4[2 \mathrm{M}+\mathrm{H}]^{+}, 673.4[2 \mathrm{M}+\mathrm{Na}]^{+}$, $689.5[2 \mathrm{M}+\mathrm{K}]^{+}, 324.3[\mathrm{M}-\mathrm{H}]^{-}, 649.4[2 \mathrm{M}-\mathrm{H}]^{-}$.

HRMS (ESI): calc. for $\left[\mathrm{C}_{19} \mathrm{H}_{20} \mathrm{NO}_{4}\right]^{+}\left([\mathrm{M}+\mathrm{H}]^{+}\right): 326.3611$, found: 326.3619; calc. for $\left[\mathrm{C}_{19} \mathrm{H}_{19} \mathrm{NO}_{4} \mathrm{Na}\right]^{+}\left([\mathrm{M}+\mathrm{Na}]^{+}\right): 348.3579$, found: 348.3575; calc. for $\left[\mathrm{C}_{19} \mathrm{H}_{18} \mathrm{NO}_{4}\right]^{-}\left([\mathrm{M}-\mathrm{H}]^{-}\right): 324.3513$, found: 324.3516

\subsubsection{4(S)-3-(((9H-Fluoren-9-ylmethoxy)carbonyl)amino)-4-( $\mathrm{N}$-((tert-butoxy)carbonyl)indol-}

\section{3-yl)butanoic Acid}

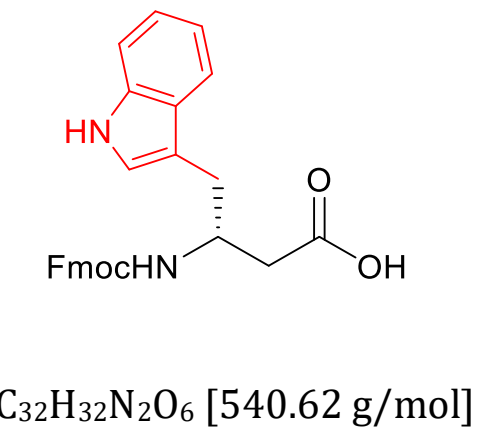

Synthetic Procedure: The N(Fmoc)- $\beta^{3}-\mathrm{D}-\mathrm{Trp}(\mathrm{Boc})-\mathrm{OH}$ was efficiently synthesized following the G.P - 1 and G.P - 2 (see Table -1 for purification strategy and overall yield).

1H NMR (300.0 MHz, $\mathbf{C D C l}_{3}$ ): $\delta 1.65$ (s, 9H, $t$-Bu), 2.33-2.45 (m, 2H, CH${ }_{2} \mathrm{CO}$ ), 2.61-2.85 (m, 1H, CHN), 3.07-3.19 (m, 2H, CH2-indol-3-yl), 4.14-4.25 (m, 1H, $\left.\mathrm{CHCH}_{2} \mathrm{O}\right), 4.24-4.56$ (m, 2H, $\mathrm{CH}_{2} \mathrm{O}$ ), $5.66(\mathrm{~d}, J=8.4 \mathrm{~Hz}, 1 \mathrm{H}, \mathrm{NH}), 7.24-7.60\left(\mathrm{~m}, 10 \mathrm{H}_{\mathrm{Ar}}\right), 7.70-8.12(\mathrm{~m}$, $\left.3 \mathrm{H}_{\mathrm{Ar}}\right)$. 
ESI-MS: $(m / z): 541.6[\mathrm{M}+\mathrm{H}]^{+}, 563.6[\mathrm{M}+\mathrm{Na}]^{+}, 579.6[\mathrm{M}+\mathrm{K}]^{+}, 1082.6[2 \mathrm{M}+\mathrm{H}]^{+}, 1104.6[2 \mathrm{M}+\mathrm{Na}]^{+}$, $1120.5[2 \mathrm{M}+\mathrm{K}]^{+}, 539.6[\mathrm{M}-\mathrm{H}]^{-}, 1080.6[2 \mathrm{M}-\mathrm{H}]^{-}$.

HRMS (ESI): calc. for $\left[\mathrm{C}_{32} \mathrm{H}_{33} \mathrm{~N}_{2} \mathrm{O}_{6}\right]^{+}\left([\mathrm{M}+\mathrm{H}]^{+}\right)$: 541.6297, found: 541.6290; calc. for $\left[\mathrm{C}_{32} \mathrm{H}_{32} \mathrm{~N}_{2} \mathrm{O}_{6} \mathrm{Na}\right]^{+}\left([\mathrm{M}+\mathrm{Na}]^{+}\right): 563.6272$, found: 563.6266; calc. for $\left[\mathrm{C}_{32} \mathrm{H}_{31} \mathrm{~N}_{2} \mathrm{O}_{6}\right]^{-}\left([\mathrm{M}-\mathrm{H}]^{-}\right.$ ):539.6698, found: 539.6689

\subsubsection{5(3S)-7-(((1,1-Dimethylethoxy)carbonyl)amino)-3-(((9H-fluoren-9-} ylmethoxy)(arbonyl)amino)heptanoic acid

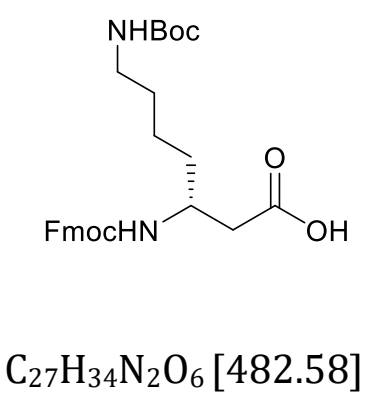

Synthetic Procedure: The N(Fmoc)- $\beta^{3}$-D-Lys(Boc)-OH was efficiently synthesized following the G.P - 1 and G.P - 2 (see Table - 1 for purification strategy and overall yield).

1H NMR (300 MHz, $\mathbf{C D C l}_{3}$ ): $\delta$ 1.15-1.41 (m, 15H, 3CH3, 3CH$)$, 2.29-2.37 (m, 2H, $2 \alpha-\mathrm{CH}_{2}$ ), 2.85$2.97\left(\mathrm{~m}, 2 \mathrm{H}, \mathrm{CH}_{2}\right), 3.61-3.76(\mathrm{~m}, 1 \mathrm{H}, \beta-\mathrm{CH}), 4.19-4.37\left(\mathrm{~m}, 3 \mathrm{H}\right.$, fluorenyl- $\mathrm{CH}_{2}$, fluorenyl $\left.-\mathrm{CH}\right), 6.65$ (s, 1H, NH), $7.13(\mathrm{~d}, 1 \mathrm{H}, J=8.7 \mathrm{~Hz}, \mathrm{NH}), 7.22-7.31(\mathrm{~m}, 2 \mathrm{H}, 2$ fluorenyl $-\mathrm{CH}), 7.45$ (t, J = 7.4 Hz, 2H, 2 fluorenyl -CH), 7.65 (d, 2H, J= 7.5 Hz, 2 fluorenyl -CH), 7.87 (d, J = 7.4 Hz, 2H, 2 fluorenyl -CH), $12.06\left(\mathrm{~S}_{\mathrm{br}}, 1 \mathrm{H}, \mathrm{COOH}\right)$.

ESI-MS: $(m / z): 483.6[\mathrm{M}+\mathrm{H}]^{+}, 505.5[\mathrm{M}+\mathrm{Na}]^{+}, 521.5[\mathrm{M}+\mathrm{K}]^{+}, 966.5[2 \mathrm{M}+\mathrm{H}]^{+}, 988.5[2 \mathrm{M}+\mathrm{Na}]^{+}$, 1004.6[2M+K] $]^{+}, 481.6[\mathrm{M}-\mathrm{H}]^{-}, 964.5[2 \mathrm{M}-\mathrm{H}]^{-}$.

HRMS (ESI): calc. for $\left[\mathrm{C}_{27} \mathrm{H}_{35} \mathrm{~N}_{2} \mathrm{O}_{6}\right]^{+}\left([\mathrm{M}+\mathrm{H}]^{+}\right): 483.6005$, found: 483.6014; calc. for $\left[\mathrm{C}_{27} \mathrm{H}_{34} \mathrm{~N}_{2} \mathrm{O}_{6} \mathrm{Na}\right]^{+}\left([\mathrm{M}+\mathrm{Na}]^{+}\right): 505.5892$, found: 505.5882; calc. for $\left[\mathrm{C}_{27} \mathrm{H}_{33} \mathrm{~N}_{2} \mathrm{O}_{6}\right]^{-}\left([\mathrm{M}-\mathrm{H}]^{-}\right.$ ):481.6121, found: 481.6129 


\subsubsection{6 (S)-3-((tert-butoxycarbonyl)amino)-4-methylpentanoic acid}

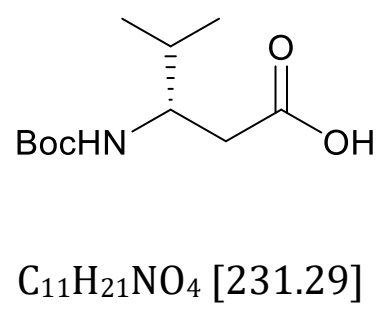

Synthetic Procedure: The N(Boc)- $\beta^{3}$-D-Val-OH was efficiently synthesized following the G.P - 1 and G.P - 2 (see Table -1 for purification strategy and overall yield).

1H NMR (300 MHz, $\mathbf{C D C l}_{3}$ ): $\delta 4.93(\mathrm{~d}, J=9.1 \mathrm{~Hz}, 1 \mathrm{H} ; \mathrm{NH}), 3.83-3.69(\mathrm{~m}, 1 \mathrm{H} ; \mathrm{NHCH}), 2.63-2.40(\mathrm{~m}$, $\left.2 \mathrm{H} ; \mathrm{CHCH}_{2}\right), 1.94-1.74(\mathrm{~m}, 1 \mathrm{H} ; \mathrm{NHCHCH}), 1.44\left(\mathrm{~s}, 9 \mathrm{H} ; \mathrm{C}\left(\mathrm{CH}_{3}\right)_{3}\right), 0.93(\mathrm{~d}, J=6.7 \mathrm{~Hz}, 6 \mathrm{H}$; $\left.\mathrm{CHC}\left(\mathrm{CH}_{3}\right)_{2}\right)$.

ESI-MS: $(\mathrm{m} / \mathrm{z}): 232.3[\mathrm{M}+\mathrm{H}]^{+}, 254.3[\mathrm{M}+\mathrm{Na}]^{+}, 270.3[\mathrm{M}+\mathrm{K}]^{+}, 463.2[2 \mathrm{M}+\mathrm{H}]^{+}, 485.2[2 \mathrm{M}+\mathrm{Na}]^{+}$

HRMS (ESI): calc. for $\left[\mathrm{C}_{11} \mathrm{H}_{22} \mathrm{NO}_{4}\right]^{+}\left([\mathrm{M}+\mathrm{H}]^{+}\right)$: 232.3166, found: 232.3159; calc. for $\left[\mathrm{C}_{11} \mathrm{H}_{22} \mathrm{NO}_{4} \mathrm{Na}\right]^{+}\left([\mathrm{M}+\mathrm{Na}]^{+}\right): 254.3156$, found: 254.3150 .

\subsubsection{7 (S)-3-((tert-butoxycarbonyl)amino)-5-methylhexanoic acid}

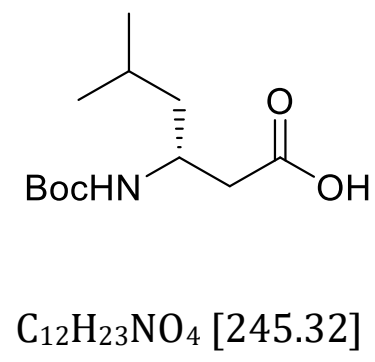

Synthetic Procedure: The N(Fmoc)- $\beta^{3}$-D-Leu-OH was efficiently synthesized following the G.P 1 and G.P - 2 (see Table -1 for purification strategy and overall yield). 
${ }^{1} \mathbf{H}$ NMR (300 MHz, $\left.\mathbf{C D C l}_{3}\right): \delta 5.02(\mathrm{~d}, J(\mathrm{H}, \mathrm{H})=8.6 \mathrm{~Hz}, 1 \mathrm{H} ; \mathrm{NH}), 4.07-3.89(\mathrm{~m}, 1 \mathrm{H}$;

$\mathrm{NHCH}$ ), 2.64-2.40 (m, 2H; $\left.\mathrm{CH}_{2} \mathrm{CO}\right), 1.78-1.56\left(\mathrm{~m}, 1 \mathrm{H} ; \mathrm{CHC}\left(\mathrm{CH}_{3}\right)_{2}\right), 1.57-1.37$ (m, 1H;

CHHCHC(CH$\left.)_{2}\right), 1.44\left(\mathrm{~s}, 9 \mathrm{H} ; \mathrm{C}\left(\mathrm{CH}_{3}\right)_{3}\right), 1.38-1.19\left(\mathrm{~m}, 1 \mathrm{H} ; \mathrm{CHHCHC}\left(\mathrm{CH}_{3}\right)_{2}\right), 0.92(\mathrm{~d}, J(\mathrm{H}, \mathrm{H})=6.5$ $\left.\mathrm{Hz}, 6 \mathrm{H} ; \mathrm{C}\left(\mathrm{CH}_{3}\right)_{2}\right)$

ESI-MS: $(m / z): 246.3[\mathrm{M}+\mathrm{H}]^{+}, 268.2[\mathrm{M}+\mathrm{Na}]^{+}, 284.3[\mathrm{M}+\mathrm{K}]^{+}, 491.3[2 \mathrm{M}+\mathrm{H}]^{+}, 513.3[2 \mathrm{M}+\mathrm{Na}]^{+}$, $529.3[2 \mathrm{M}+\mathrm{K}]^{+}$

HRMS (ESI): calc. for $\left[\mathrm{C}_{12} \mathrm{H}_{24} \mathrm{NO}_{4}\right]^{+}\left([\mathrm{M}+\mathrm{H}]^{+}\right)$: 246.3265, found: 246.3258; calc. for $\left[\mathrm{C}_{12} \mathrm{H}_{23} \mathrm{NO}_{4} \mathrm{Na}\right]^{+}\left([\mathrm{M}+\mathrm{Na}]^{+}\right): 268.2977$, found: 268.3058

\subsubsection{Tert-butyl (S)-(4-fluoro-4-oxobutan-2-yl)carbamate}

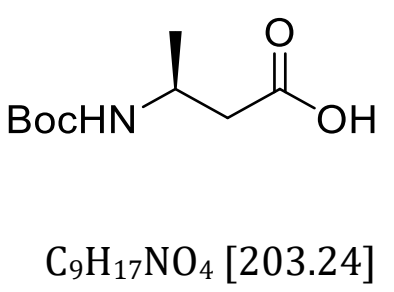

$\mathrm{C}_{9} \mathrm{H}_{17} \mathrm{NO}_{4}[203.24]$

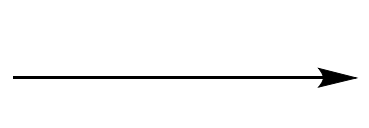

$\mathrm{C}_{9} \mathrm{H}_{16} \mathrm{FNO}_{3}[205.23]$

Synthetic Procedure: The N(Boc)-protected $\beta^{3}$-D-Ala-OH (1 eq., 3.0 g, $14.7 \mathrm{mmol}$ ) was dissolved in anhydrous dichloromethane $(8 \mathrm{~mL} / 1 \mathrm{mmol})$ and was treated with pyridine (1 eq., $1.1 \mathrm{~mL}, 14.7$ mmol) and cyanuric fluoride (0.5 eq., $0.63 \mathrm{~mL}, 7.35 \mathrm{mmol})$, respectively. The reaction mixture was stirred for $3 \mathrm{~h}$ at $-30^{\circ} \mathrm{C}$ temperature in a cryostat $\left(-30^{\circ} \mathrm{C}\right.$ temperature was used in order to avoid the formation of Leuch's anhydride). The reaction was monitored by TLC and on completion, it was was diluted with dichloromethane and washed twice with a small amount of ice water. The organic fraction was dried under $\mathrm{Na}_{2} \mathrm{SO}_{4}$ and the excess solvent was removed under reduced pressure. The resulting acid fluoride was used for the reaction without further purification.

${ }^{1}$ H NMR (300 MHz, $\left.\mathbf{C D C l}_{3}\right): \delta 1.29(d, J=6.6 \mathrm{~Hz}, 3 \mathrm{H}) ; 1.44(s, 9 \mathrm{H}) ; 2.62-2.83(m, 2 \mathrm{H})$; $3.98-4.15(m, 1 \mathrm{H}) ; 4.70$ (br.s, NH). 
HRMS (ESI): calc. for $\left[\mathrm{C}_{9} \mathrm{H}_{17} \mathrm{FNO}_{3}\right]^{+}\left([\mathrm{M}+\mathrm{H}]^{+}\right)$: 206.2338, found: 206.2346; calc. for $\left[\mathrm{C}_{9} \mathrm{H}_{16} \mathrm{FNO}_{3} \mathrm{Na}\right]^{+} \quad\left([\mathrm{M}+\mathrm{Na}]^{+}\right): \quad 228.2544$, found: $228.2537 ;$ calc. for $\left[\mathrm{C}_{9} \mathrm{H}_{16} \mathrm{FNO}_{3} \mathrm{~K}\right]^{+}$ $\left([\mathrm{M}+\mathrm{K}]^{+}\right): 244.2144$, found: 244.2150

\subsubsection{9 tert-butyl-(S)-(4-fluoro-4-oxo-1-phenylbutan-2-yl)carbamate}

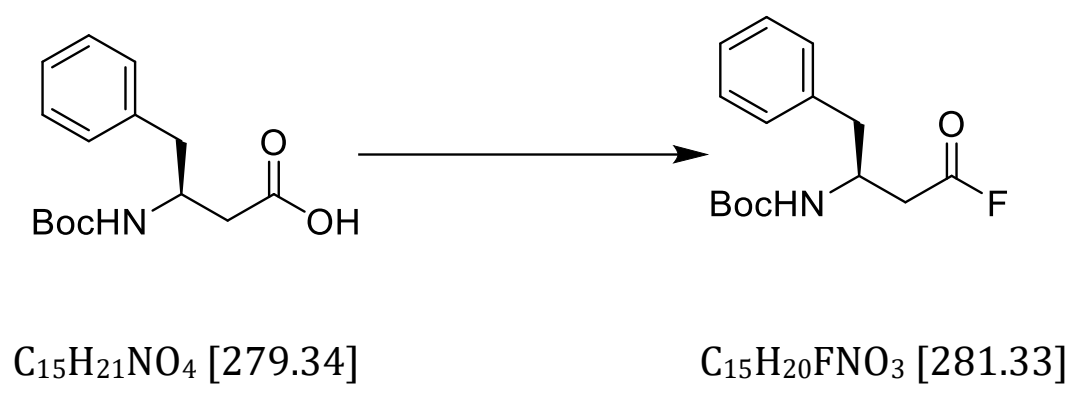

Synthetic procedure: The N(Boc)-protected $\beta^{3}$-D-Phe-OH (1 eq., 3.0 g, $10.73 \mathrm{mmol}$ ) was dissolved in anhydrous dichloromethane $(8 \mathrm{~mL} / 1 \mathrm{mmol})$ and was treated with pyridine (1 eq., $0.86 \mathrm{~mL}, 10.73 \mathrm{mmol}$ ) and cyanuric fluoride (0.5 eq., $0.46 \mathrm{~mL}, 5.36 \mathrm{mmol})$, respectively. The reaction mixture was stirred for $3 \mathrm{~h}$ at $-30^{\circ} \mathrm{c}$ temperature in a cryostat $\left(-30^{\circ} \mathrm{C}\right.$ temperature was used in order to avoid the formation of Leuch's anhydride). The reaction was monitored by TLC and on completion, it was was diluted with dichloromethane and washed twice with a small amount of ice water. The organic fraction was dried under $\mathrm{Na}_{2} \mathrm{SO}_{4}$ and the excess solvent was removed under reduced pressure. The resulting acid fluoride was used for the reaction without further purification.

1H NMR (300 MHz, $\left.\mathbf{C D C l}_{3}\right): \delta 1.42(s, 9 \mathrm{H}) ; 2.55$ - 3.05 (m, $\left.4 \mathrm{H}\right) ; 4.18$ (br.s, $1 \mathrm{H}$ ); 4.81 (br.s, NH); $7.16-7.36(m, 5 \mathrm{H})$.

ESI-MS: $(\mathrm{m} / \mathrm{z}): 282.3[\mathrm{M}+\mathrm{H}]^{+}, 304.3[\mathrm{M}+\mathrm{Na}]^{+}, 320.2[\mathrm{M}+\mathrm{K}]^{+}, 563.3[2 \mathrm{M}+\mathrm{H}]^{+}$

HRMS (ESI): calc. for $\left[\mathrm{C}_{15} \mathrm{H}_{21} \mathrm{FNO}_{3}\right]^{+}\left([\mathrm{M}+\mathrm{H}]^{+}\right)$: 282.3378, found: 282.3370; calc. for $\left[\mathrm{C}_{15} \mathrm{H}_{20} \mathrm{FNO}_{3} \mathrm{Na}\right]^{+}\left([\mathrm{M}+\mathrm{Na}]^{+}\right): 304.3455$, found: 304.3447 


\subsubsection{Benzyl-(S)-(1-fluoro-4-methyl-1-oxopentan-3-yl)carbamate}

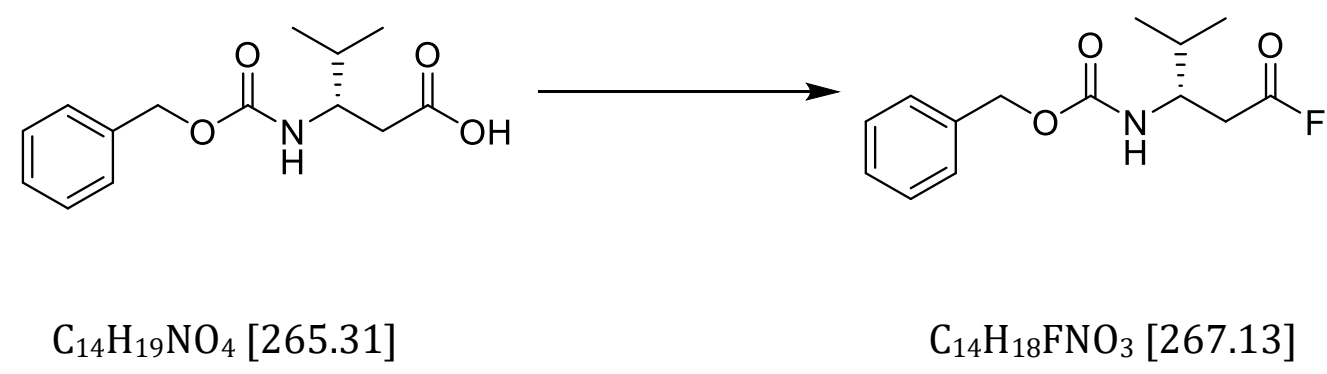

Synthetic procedure: The $\mathrm{N}(\mathrm{Cbz})$-protected $\beta^{3}$-D-Val-OH (1 eq., 3.0 g, $11.3 \mathrm{mmol}$ ) was dissolved in anhydrous dichloromethane $(8 \mathrm{~mL} / 1 \mathrm{mmol})$ and was treated with pyridine (1 eq., $0.91 \mathrm{~mL}, 11.3 \mathrm{mmol})$ and cyanuric fluoride $(0.5$ eq., $0.48 \mathrm{~mL}, 5.65 \mathrm{mmol})$, respectively. The reaction mixture was stirred for $3 \mathrm{~h}$ at $-30^{\circ} \mathrm{c}$ temperature in a cryostat $\left(-30^{\circ} \mathrm{C}\right.$ temperature was used in order to avoid the formation of Leuch's anhydride). The reaction was monitored by TLC and on completion, it was was diluted with dichloromethane and washed twice with a small amount of ice water. The organic fraction was dried under $\mathrm{Na}_{2} \mathrm{SO}_{4}$ and the excess solvent was removed under reduced pressure. The resulting acid fluoride was used for the reaction without further purification.

1H NMR (300 MHz, CDCl 3 ): $\delta 0.93-1.02(m, 6 \mathrm{H}) ; 1.82-2.02(m, 1 \mathrm{H}) ; 2.66-2.87(m, 2 \mathrm{H}), 3.78$ - 3.93 (m, 1 H); 4.95 (br.s, NH); 5.09 (br.s, 2 H); 7.33 - 7.40 (m, 5 H).

ESI-MS: $(m / z): 268.1[\mathrm{M}+\mathrm{H}]^{+}, 290.0[\mathrm{M}+\mathrm{Na}]^{+}, 306.1[\mathrm{M}+\mathrm{K}]^{+}, 535.3[2 \mathrm{M}+\mathrm{H}]^{+}$

HRMS (ESI): calc. for $\left[\mathrm{C}_{14} \mathrm{H}_{19} \mathrm{FNO}_{3}\right]^{+}\left([\mathrm{M}+\mathrm{H}]^{+}\right):$268.1326, found: 268.1329; calc. for $\left[\mathrm{C}_{14} \mathrm{H}_{18} \mathrm{FNO}_{3} \mathrm{Na}\right]^{+}\left([\mathrm{M}+\mathrm{Na}]^{+}\right): 290.0922$, found: 290.0930 


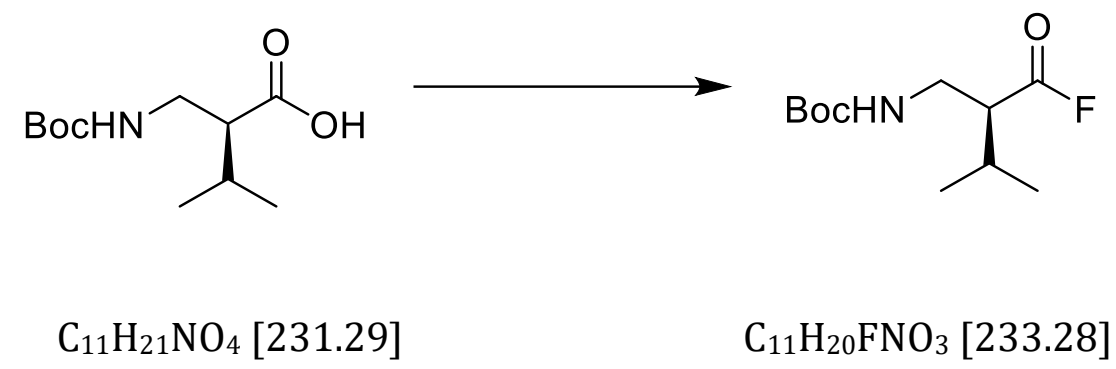

Synthetic procedure: The N(Boc)-protected $\beta^{2}$-D-Val-OH (1 eq., 3.0 g, $12.9 \mathrm{mmol}$ ) was dissolved in anhydrous dichloromethane $(8 \mathrm{~mL} / 1 \mathrm{mmol})$ and was treated with pyridine (1 eq., $1.04 \mathrm{~mL}$, $12.9 \mathrm{mmol})$ and cyanuric fluoride $(0.5$ eq., $0.55 \mathrm{~mL}, 6.4 \mathrm{mmol})$, respectively. The reaction mixture was stirred for $3 \mathrm{~h}$ at $-30^{\circ} \mathrm{C}$ temperature in a cryostat $\left(-30^{\circ} \mathrm{C}\right.$ temperature was used in order to avoid the formation of Leuch's anhydride). The reaction was monitored by TLC and on completion, it was was diluted with dichloromethane and washed twice with a small amount of ice water. The organic fraction was dried under $\mathrm{Na}_{2} \mathrm{SO}_{4}$ and the excess solvent was removed under reduced pressure. The resulting acid fluoride was used for the reaction without further purification.

${ }^{1} \mathrm{H}$ NMR (300 MHz, CDCl 3$): \delta 1.04(d, J=6.6 \mathrm{~Hz}, 6 \mathrm{H}) ; 1.44(s, 9 \mathrm{H}) ; 1.99-2.15(m, 1 \mathrm{H})$;

2.75 (br.s, $1 \mathrm{H}), 3.13-3.32$ (m, $1 \mathrm{H}) ; 3.42-3.60$ (m, $1 \mathrm{H}) ; 4.81$ (br.s, NH).

ESI-MS: $(m / z): 234.2[\mathrm{M}+\mathrm{H}]^{+}, 256.3[\mathrm{M}+\mathrm{Na}]^{+}, 272.2[\mathrm{M}+\mathrm{K}]^{+}, 467.5[2 \mathrm{M}+\mathrm{H}]^{+}$

HRMS (ESI): calc. for $\left[\mathrm{C}_{11} \mathrm{H}_{21} \mathrm{FNO}_{3}\right]^{+}\left([\mathrm{M}+\mathrm{H}]^{+}\right): 234.2811$, found: 234.2820; calc. for $\left[\mathrm{C}_{11} \mathrm{H}_{20} \mathrm{FNO}_{3} \mathrm{Na}\right]^{+}\left([\mathrm{M}+\mathrm{Na}]^{+}\right): 256.3077$, found: 256.3084

\subsubsection{2(S)-3-tert-Butoxycarbonylamino-4-phenyl-butyricacid[4}

$(3,3,4,4,5,5,6,6,7,7,8,8,9,9,10,10,10$-heptadecafluorodecyl)- benzyl ester]<smiles>C[C@H](CC(=O)O)NC(=O)OCc1ccccc1</smiles><smiles>CC(C)N[C@@H](C)CC(=O)OCc1ccc(CC[18F])cc1</smiles> 


$$
\mathrm{C}_{9} \mathrm{H}_{17} \mathrm{NO}_{4}[203.24] \quad \mathrm{C}_{26} \mathrm{H}_{26} \mathrm{~F}_{17} \mathrm{NO}_{4}[739.47]
$$

Synthetic preocedure: The N-protected amino acid (1 eq., 2.0 g., $9.8 \mathrm{mmol}$ ) was first dissolved in a solvent mixture composed of dichloromethane $(80 \mathrm{~mL})$ and trifluorobenzene $(40 \mathrm{~mL})$ in $2: 1$ ratio and DMAP (0.15 eq., 0.17 g., $1.4 \mathrm{mmol})$, the heavy fluorous tag [4 (3,3,4,4,5,5,6,6,7,7,8,8,9,9,10,10,10-heptadecafluorodecyl)benzylalcohol] [0.7 eq., 3.8 g., 6.8 mmol) and diisopropylcarbodiimide (1.1 eq., 2.2 g., $10.7 \mathrm{mmol}$ ) were added to the reaction mixture, respectively. The reaction mixture was stirred overnight at room temperature. Next day, the reaction mixture was diluted with dichloromethane $(250 \mathrm{~mL})$ and washed with aqueous $\mathrm{KH}_{2} \mathrm{PO}_{4}$ solution $(1 \mathrm{M}, 100 \mathrm{~mL})$, aqueous $\mathrm{K}_{2} \mathrm{HPO}_{4}$ solution $(1 \mathrm{M}, 100 \mathrm{~mL})$, and brine $(100 \mathrm{~mL})$. The combined organic fractions were dried over $\mathrm{Na}_{2} \mathrm{SO}_{4}$ and the excess solvent was removed under reduced pressure.

1H NMR (300 MHz, $\left.\mathbf{C D C l}_{3}\right): \delta 1.41(s, 9 \mathrm{H}) ; 2.26-2.46(m, 2 \mathrm{H}) ; 2.48,2.56(J=5.8 \mathrm{~Hz}, 16.2 \mathrm{~Hz}, 1$ H each); $2.80(d d, J=7.7 \mathrm{~Hz}, 13.7 \mathrm{~Hz}, 1 \mathrm{H}) ; 2.88-2.97(m, 1 \mathrm{H}) ; 4.18$ (br. $d, J=6.3 \mathrm{~Hz}, 1 \mathrm{H}) ; 5.03$ $(d, J=8.1 \mathrm{~Hz}, \mathrm{NH}) ; 5.09-5.13(J=12.2 \mathrm{~Hz}, 1 \mathrm{H}$ each$) ; 7.11-7.36(m, 9 \mathrm{H})$.

${ }^{13} \mathrm{C}$ NMR (75 MHz, $\left.\mathbf{C D C l}_{3}\right): \delta 26.2\left(\mathrm{CH}_{2}\right), 28.3\left(\mathrm{CMe}_{3}\right), 32.9\left(t, J=22 \mathrm{~Hz}, \mathrm{CH}_{2}\right), 37.7,40.3\left(2 \times \mathrm{CH}_{2}\right)$, $48.8(\mathrm{CH}), 66.1\left(\mathrm{CH}_{2}\right), 79.4(\mathrm{C}), 126.6,128.5,128.6,128.9,129.4(5 \mathrm{x} \mathrm{CH}), 134.2,137.6,139.4$, 155.1, 171.5 ( 5 x C).

ESI-MS: $(\mathrm{m} / \mathrm{z}): 740.4[\mathrm{M}+\mathrm{H}]^{+}, 762.5[\mathrm{M}+\mathrm{Na}]^{+}, 778.5[\mathrm{M}+\mathrm{K}]^{+}, 1480.4[2 \mathrm{M}+\mathrm{H}]^{+}$

HRMS (ESI): calc. for $\left[\mathrm{C}_{26} \mathrm{H}_{27} \mathrm{~F}_{17} \mathrm{NO}_{4}\right]^{+}\left([\mathrm{M}+\mathrm{H}]^{+}\right)$: 740.4688, found: 740.4679; calc. for $\left[\mathrm{C}_{26} \mathrm{H}_{26} \mathrm{~F}_{17} \mathrm{NO}_{4} \mathrm{Na}\right]^{+}\left([\mathrm{M}+\mathrm{Na}]^{+}\right): 762.5076$, found: 762.5072

\subsubsection{3 (S)-N-benzyl-N-(methoxymethyl)-1-phenylethan-1-amine}

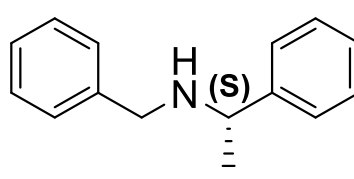

$\mathrm{C}_{15} \mathrm{H}_{17} \mathrm{~N}$ [211.319]

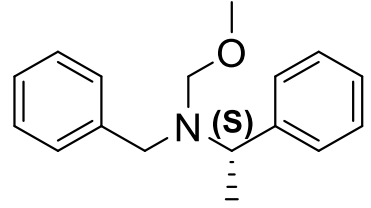

$\mathrm{C}_{17} \mathrm{H}_{21} \mathrm{NO}[255.36]$ 
Synthetic procedure: Following the method described by Chi et al.,[6,7] $(S)$ - $N$-benzyl-Rmethylbenzylamine $(0.023 \mathrm{~mol}, 5 \mathrm{~mL})$ was dissolved in $20 \mathrm{~mL}$ of anhydrous $\mathrm{MeOH}$ in a $100 \mathrm{~mL}$ round bottom flask at room temperature. Paraformaldehyde (2.5 eq, $0.058 \mathrm{~mol}, 1.77 \mathrm{~g}$ ) followed by anhydrous $\mathrm{K}_{2} \mathrm{CO}_{3}$ (3.3 g) and anhydrous $\mathrm{Na}_{2} \mathrm{SO}_{4}(3.3 \mathrm{~g}$ ) were added to the flask. The reaction was continued initially for $1 \mathrm{hr}$ at room temperature and TLC monitoring indicated completion of the reaction within 30 minutes. Nevertheless, the reaction mixture was stirred at room temperature overnight (12 hrs) to ensure water produced during the reaction was completely absorbed by anhydrous $\mathrm{K}_{2} \mathrm{CO}_{3}$ and anhydrous $\mathrm{Na}_{2} \mathrm{SO}_{4}$. Afterwards the mixture was filtered through a pad of anhydrous $\mathrm{Na}_{2} \mathrm{SO}_{4}$ to eliminate any solid material left unreacted and the remaining solids were washed thoroughly by anhydrous $\mathrm{MeOH}(50 \mathrm{~mL}$ ) and filtered. All filtrates were combined and the milky mixture was concentrated under reduced pressure to yield a mixture of oil and white solid. This mixture was again mixed with anhydrous $\mathrm{Et}_{2} \mathrm{O}(30 \mathrm{~mL})$ and filtered through a pad of anhydrous $\mathrm{Na}_{2} \mathrm{SO}_{4}$, so that the white solid (paraformaldehyde) remains as solid in $\mathrm{Et}_{2} \mathrm{O}$ and were easily separated from the oily organic phase. The filtrate is again concentrated under reduced pressure to yield a clear oil (yield: 63\%).

1H NMR (300 MHz, $\mathbf{C D C l}_{3}$ ): $\delta$ 7.28-7.41 (m, 10H), 4.07 and $4.11(\mathrm{~s}, 1 \mathrm{H}), 4.03(\mathrm{q}, J=6.2 \mathrm{~Hz}, 1 \mathrm{H})$, 3.86 and $3.86(\mathrm{~s}, 1 \mathrm{H}), 3.71-3.77(\mathrm{~m}, 2 \mathrm{H}), 3.15(\mathrm{~s}, 3 \mathrm{H}), 1.42(\mathrm{~d}, J=6.5 \mathrm{~Hz}, 3 \mathrm{H})$.

${ }^{13}$ C NMR (75 MHz, CDCl3): $\delta$ 144.3, 139.0 127.0, 128.5, 128.2, 127.5, 127.3, 127.0, 82.4, 59.3, $55.7,52.3,20.3$.

ESI-MS: The compound decomposed to corresponding amine under the conditions of ESI-MS analysis. Only a trace amount of $\mathrm{N}, \mathrm{O}$-acetal was detected at $278.5\left(\mathrm{M}+\mathrm{Na}^{+}\right)$

\subsubsection{4 (S)-2-((benzyl((S)-1-phenylethyl)amino)methyl)-3-methylbutan-1-ol}




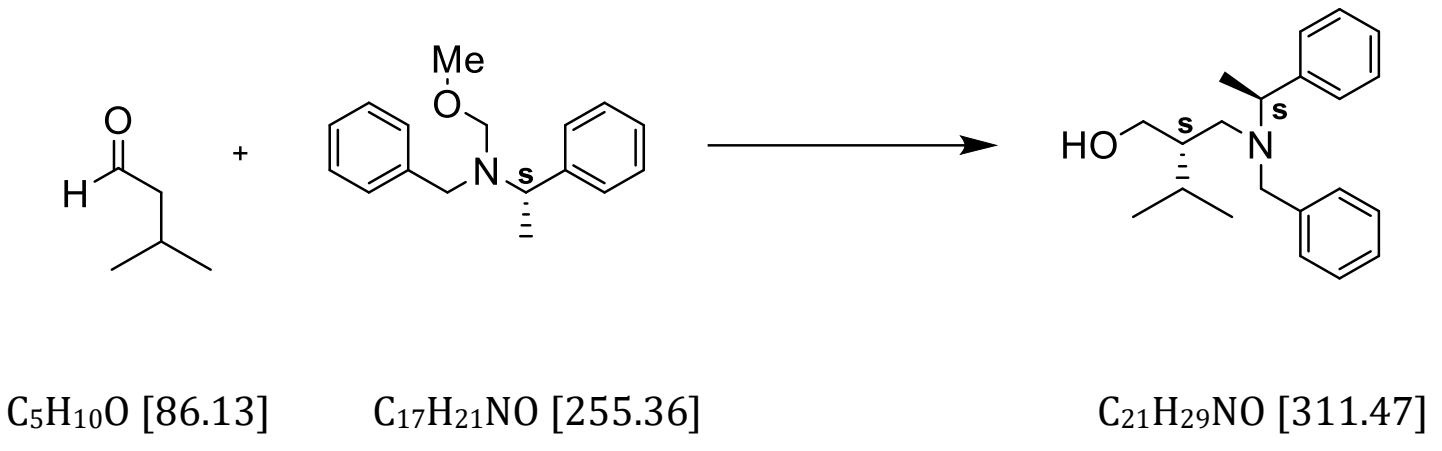

Synthetic procedure: L-Proline ( 0.2 eq., $1.1 \mathrm{~g}$., $10.3 \mathrm{mmol}$ ) was taken in a $250 \mathrm{~mL}$ round bottom flask, anhydrous DMF (110 mL) was added to it, and the catalyst mixture was cooled to $-25^{\circ} \mathrm{C}$ in a cryostat. To the cooled catalyst mixture was added the isovaleraldehyde (2 eq., $11.08 \mathrm{~mL}, 103.3$ mmol) and the mixture was stirred for $1 \mathrm{hr}$. N,O-Acetal (1 eq., $13.2 \mathrm{~g}$., $51.6 \mathrm{mmol}$ ) was then added, and the mixture was stirred at $-25{ }^{\circ} \mathrm{C}$ for $24 \mathrm{~h}$. The reaction was monitored using TLC (EtOAc/Pentane (1:5).

Excess $\mathrm{NaBH}_{4}$ (3 eq., 5.8 g., $155.07 \mathrm{mmol}$ ) and $40 \mathrm{~mL}$ of $\mathrm{MeOH}$ were then added slowly to the reaction mixture. The mixture was stirred for 10 minutes; the $-25{ }^{\circ} \mathrm{C}$ bath or the cryostat was then replaced by an ice bath and was subsequently stirred for an additional $30 \mathrm{~min}$. The mixture was then slowly poured into a $250 \mathrm{~mL}$ beaker containing $50 \mathrm{~mL}$ of saturated $\mathrm{NH}_{4} \mathrm{Cl}$ at $0{ }^{\circ} \mathrm{C}$ to quench excess $\mathrm{NaBH}_{4}$. The resulting mixture was filtered and the solid was washed several times with $\mathrm{Et}_{2} \mathrm{O}$. The filtrate was transferred to a separating funnel and the two phases were separated. The aqueous phase was extracted with $\mathrm{Et}_{2} \mathrm{O}$ until TLC indicated that product had been removed into the organic phase. The $\mathrm{Et}_{2} \mathrm{O}$ layers were combined, washed with water and then brine, dried over $\mathrm{MgSO}_{4}$, filtered, and concentrated to give crude diastereomeric mixture of (R/S)-2((benzyl((S)-1-phenylethyl)amino)methyl)-4-methylpentan-1-ol (the remaining DMF was removed under vacuum). The major diastereomer $(\boldsymbol{s}, \boldsymbol{s})$ was isolated via column chromatography using EtOAc/Pentane (1:10) with 29\% yield.

${ }^{1}$ H NMR (300 MHz, $\left.\mathbf{C D C l}_{3}\right): \delta$ 7.37-7.23 (m, 10H), 5.07-5.04 (br, 1H), $4.05(\mathrm{q}, J=6.6 \mathrm{~Hz}, 1 \mathrm{H}), 3.95$ \& $3.91(\mathrm{~s}, 1 \mathrm{H}), 3.62-3.56(\mathrm{br}, 1 \mathrm{H}), 3.37 \& 3.33(\mathrm{~s}, 1 \mathrm{H}), 3.10-3.04(\mathrm{~m}, 1 \mathrm{H}), 2.62-2.59(\mathrm{~m}, 2 \mathrm{H}), 1.78-$ $1.73(\mathrm{~m}, 1 \mathrm{H}), 1.49-1.42(\mathrm{~m}, 1 \mathrm{H}), 1.37(\mathrm{~d}, J=6.6 \mathrm{~Hz}, 3 \mathrm{H}), 0.84 \& 0.78$ (diastereotopic, $\mathrm{d}, J=6.9 \mathrm{~Hz}$ ).

${ }^{13}$ C NMR (75 MHz, CDCl3): $\delta$ 142.7, 138.9, 129.5, 128.7, 128.4, 127.5, 127.4, 66.5, 56.5, 56.1, $52.5,42.2,28.8,20.5,20.0,9.5$.

ESI-MS: $(\mathrm{m} / \mathrm{z}): 312.5[\mathrm{M}+\mathrm{H}]^{+}, 335.4[\mathrm{M}+\mathrm{Na}]^{+}, 623.5[2 \mathrm{M}+\mathrm{H}]^{+}$ 
HRMS (ESI): calc. for $\left[\mathrm{C}_{21} \mathrm{H}_{30} \mathrm{NO}\right]^{+}\left([\mathrm{M}+\mathrm{H}]^{+}\right): 312.5057$, found: 312.5048 ; calc. for $\left[\mathrm{C}_{21} \mathrm{H}_{29} \mathrm{NO} \mathrm{Na}\right]^{+}$ $\left([\mathrm{M}+\mathrm{Na}]^{+}\right): 335.4988$, found: 335.4982

\subsubsection{5 (S)-2-((benzyl((S)-1-phenylethyl)amino)methyl)-4-methylpentan-1-ol}

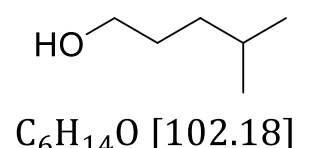

$\mathrm{C}_{6} \mathrm{H}_{14} \mathrm{O}[102.18]$

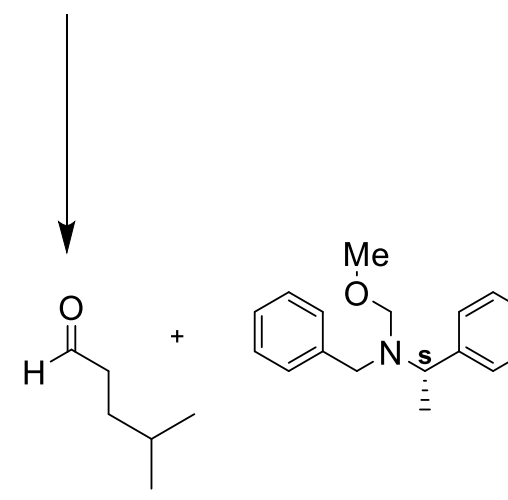

$\mathrm{C}_{6} \mathrm{H}_{12} \mathrm{O}[100.16]$

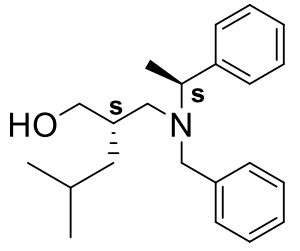

$\mathrm{C}_{22} \mathrm{H}_{31} \mathrm{NO}[325.50]$

Synthetic procedure: L-Proline (0.2 eq., 0.08g., $0.78 \mathrm{mmol}$ ) was taken in a $250 \mathrm{~mL}$ round bottom flask and anhydrous DMF (100 mL) was added to it and the catalyst mixture was cooled to $-25^{\circ} \mathrm{C}$ in a cryostat. To the cooled catalyst mixture was added the 4-methyl-1-pentanal (2 eq., 7.8 g., $78.2 \mathrm{mmol}$ ) and the mixture was stirred for $1 \mathrm{hr}$. It is worth to mention that unlike isovaleralhyde, 4-methyl-1-pentanal was commercially not available and had to be presynthesized by the reaction of pentan-1-ol (1 eq., $10 \mathrm{~mL}, 57.8 \mathrm{mmol}$ ) and $\mathrm{PhI}(\mathrm{OAc})_{2}(1.1$ eq., 34.6 g., $107.6 \mathrm{mmol}$ ) in presence of TEMPO (0.1 eq., 1.4 g., $9.6 \mathrm{mmol}$ as catalyst in DCM at room temperature. The N,O-Acetal (1 eq., 10.0 g., $39.1 \mathrm{mmol}$ ) was then added, and the mixture was stirred at $-25{ }^{\circ} \mathrm{C}$ for $24 \mathrm{~h}$. The reaction was monitored using TLC (EtOAc/Pentane (1:5).

Excess $\mathrm{NaBH}_{4}$ (3 eq., 4.4 g., $117.3 \mathrm{mmol}$ ) and $35 \mathrm{~mL}$ of $\mathrm{MeOH}$ were then added slowly to the reaction mixture. The mixture was stirred for 10 minutes; the $-25{ }^{\circ} \mathrm{C}$ bath or the cryostat was then replaced by an ice bath and was subsequently stirred for an additional $30 \mathrm{~min}$. The mixture 
was then slowly poured into a $250 \mathrm{~mL}$ beaker containing $50 \mathrm{~mL}$ of saturated $\mathrm{NH}_{4} \mathrm{Cl}$ at $0{ }^{\circ} \mathrm{C}$ to quench excess $\mathrm{NaBH}_{4}$. The resulting mixture was filtered and the solid was washed several times with $\mathrm{Et}_{2} \mathrm{O}$. The filtrate was transferred to a separating funnel and the two phases were separated. The aqueous phase was extracted with $\mathrm{Et}_{2} \mathrm{O}$ until TLC indicated that product had been removed into the organic phase. The $\mathrm{Et}_{2} \mathrm{O}$ layers were combined, washed with water and then brine, dried over $\mathrm{MgSO}_{4}$, filtered, and concentrated to give crude diastereomeric mixture of (R/S)-2((benzyl((S)-1-phenylethyl)amino)methyl)-4-methylpentan-1-ol (the remaining DMF was removed under vacuum). The major diastereomer $(\mathbf{s}, \mathbf{s})$ was isolated via column chromatography using EtOAc/Pentane (1:12) with 23\% yield.

1H NMR (300 MHz, CDCl 3 ): $\delta$ 7.34-7.26 (m, 10H), $5.12(\mathrm{br}, 1 \mathrm{H}), 4.03(\mathrm{q}, J=6.9 \mathrm{~Hz}, 1 \mathrm{H}), 3.98$ \& $3.93(\mathrm{~s}, 1 \mathrm{H}), 3.54(\mathrm{br}, 1 \mathrm{H}), 3.39 \& 3.35(\mathrm{~s}, 1 \mathrm{H}), 2.98-2.92(\mathrm{~m}, 1 \mathrm{H}), 2.56-2.52(\mathrm{~m}, 1 \mathrm{H}), 2.46-2.38(\mathrm{~m}$, $1 \mathrm{H}), 2.08-1.93(\mathrm{br}, 1 \mathrm{H}), 1.58-1.54(\mathrm{~m}, 1 \mathrm{H}), 1.35(\mathrm{~d}, J=6.9 \mathrm{~Hz}, 3 \mathrm{H}), 0.90-0.78(\mathrm{~m}, 6 \mathrm{H})$

${ }^{13}$ C NMR (75 MHz, CDCl3): $\delta$ 142.8, 138.9, 129.5, 128.7, 128.4, 128.3, 127.5, 127.3, 68.8, 56.5, $55.3,55.1,39.5,34.5,25.6,23.1,22.9,9.5$.

ESI-MS: $(\mathrm{m} / \mathrm{z}): 326.1[\mathrm{M}+\mathrm{H}]^{+}, 348.1[\mathrm{M}+\mathrm{Na}]^{+}, 652.0[2 \mathrm{M}+\mathrm{H}]^{+}$

HRMS (ESI): calc. for $\left[\mathrm{C}_{22} \mathrm{H}_{32} \mathrm{NO}\right]^{+}\left([\mathrm{M}+\mathrm{H}]^{+}\right): 326.1055$, found: 326.1063 ; calc. for $\left[\mathrm{C}_{22} \mathrm{H}_{31} \mathrm{NO} \mathrm{Na}\right]^{+}$ $\left([\mathrm{M}+\mathrm{Na}]^{+}\right): 348.1142$, found: 348.1150

\subsubsection{Tert-butyl-(S)-(2-(hydroxymethyl)-3-methylbutyl)carbamate Or (9H-fluoren-9- yl)methyl (S)-(2-(hydroxymethyl)-3-methylbutyl)carbamate}

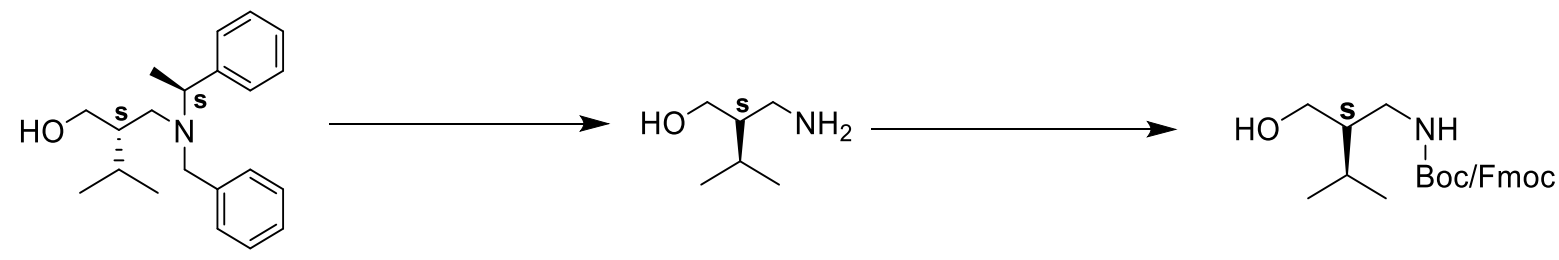


Synthetic procedure: The desired diastereomer (S)-2-((benzyl((S)-1phenylethyl)amino)methyl)-3-methylbutan-1-ol of the amino alcohol (1 eq., $2.5 \mathrm{~g}, 8.02 \mathrm{mmol}$ ) was added to the wet $\mathrm{Pd} / \mathrm{C}(0.5$ eq., $0.5 \mathrm{~g}$ ) in $25 \mathrm{~mL}$ of $\mathrm{MeOH}$ in a hydrogenation flask. The heterogeneous reaction mixture was carefully purged with Ar-stream to replace dissolved $\mathrm{O}_{2}$ by Ar followed by purging with $\mathrm{H}_{2}$-baloon. Thereafter the flask was placed in the Parr's apparatus under 3-4 $\mathrm{Bar}_{2} \mathrm{H}_{2}$ and shaken overnight at room temperature. After the hydrogenolysis was completed (indicated by TLC), the mixture was diluted with $30 \mathrm{~mL}$ of DCM and added $\mathrm{Boc}_{2} \mathrm{O}$ (1.5 eq.) or Fmoc-Osu (1.5 eq.) with DIPEA (2 eq.). The reaction mixture was stirred overnight at room temperature and on completion (checked on TLC) filtered through a pad of Celite and Florisil to remove remaining $\mathrm{Pd} / \mathrm{C}$ followed by washing the Celite and Florisil pad extensively with $\mathrm{MeOH}$. The collected fractions of filtrates were concentrated under reduced pressure. The crude product was purified by flash column chromatography with EtOAc/n-Hexane (1:12) to yield the purified $\mathrm{N}(\mathrm{Boc}) /(\mathrm{Fmoc})$-protected $\beta$-substituted $\gamma$-amino alcohol, tert-butyl (S)-(2(hydroxymethyl)-3-methylbutyl)carbamate or $\quad$ (9H-fluoren-9-yl)methyl (S)-(2(hydroxymethyl)-3-methylbutyl)carbamate with 52\% and 59\% yield, respectively.

1H NMR (300 MHz, $\mathbf{C D C l}_{3}$, Boc-protected ): $\delta 4.80$ (br, 1H), 3.69-3.66 (m, 1H), 3.55-3.51 (m, $1 \mathrm{H}), 3.38-3.35(\mathrm{~m}, 1 \mathrm{H}), 3.28-3.17(\mathrm{~m}, 2 \mathrm{H}), 1.66-1.61(\mathrm{~m}, 1 \mathrm{H}), 1.32-1.30(\mathrm{~m}, 1 \mathrm{H}), 0.95 \& 0.94$ (diastereotopic, d, $J=7.2 \mathrm{~Hz}, 6 \mathrm{H}$ ).

${ }^{13}$ C NMR (75 MHz, CDCl3, Boc protected): $\delta$ 157.9, 79.9, 61.9, 47.9, 39.7, 28.6, 27.3, 20.8, 20.7.

ESI-MS (Boc protected): $(\mathrm{m} / \mathrm{z}): 218.4[\mathrm{M}+\mathrm{H}]^{+}, 240.3[\mathrm{M}+\mathrm{Na}]^{+}, 435.4[2 \mathrm{M}+\mathrm{H}]^{+}$

HRMS (ESI, Boc protected): calc. for $\left[\mathrm{C}_{11} \mathrm{H}_{24} \mathrm{NO}_{3}\right]^{+}\left([\mathrm{M}+\mathrm{H}]^{+}\right): 218.4117$, found: 218.4110; calc. for $\left[\mathrm{C}_{11} \mathrm{H}_{23} \mathrm{NO}_{3} \mathrm{Na}\right]^{+}\left([\mathrm{M}+\mathrm{Na}]^{+}\right): 240.3144$, found: 240.3152

\subsubsection{Tert-butyl-(S)-(2-(hydroxymethyl)-4-methylpentyl)carbamate Or (9H-fluoren-9- yl)methyl (S)-(2-(hydroxymethyl)-4-methylpentyl)carbamate}




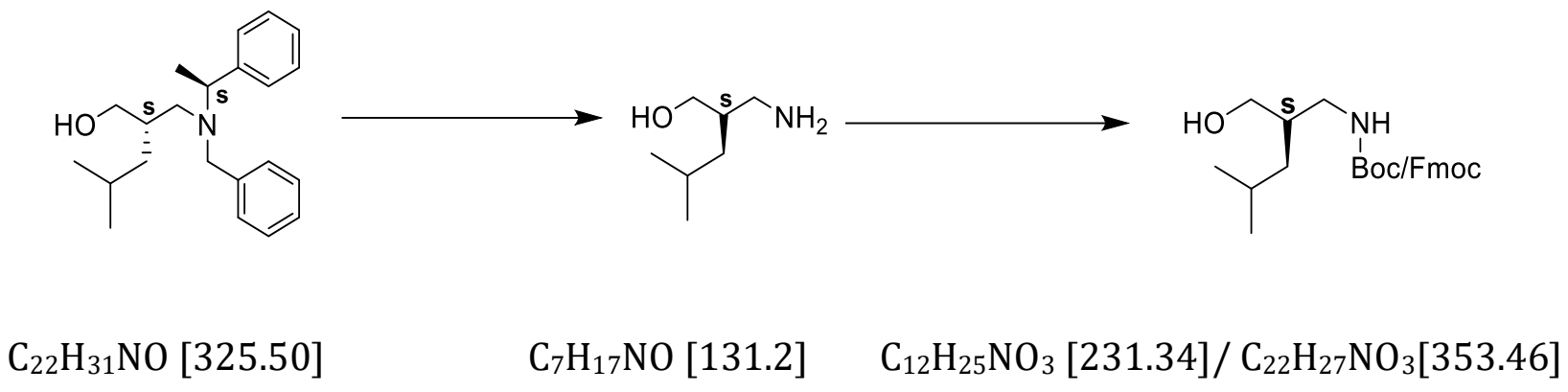

Synthetic procedure: The desired diastereomer $(S)$-2-((benzyl $((S)-1$ phenylethyl)amino)methyl)-4-methylpentan-1-ol of the amino alcohol (1 eq., $2.5 \mathrm{~g}, 7.68 \mathrm{mmol}$ ) was added to the wet $\mathrm{Pd} / \mathrm{C}(0.5$ eq., $0.5 \mathrm{~g}$ ) in $25 \mathrm{~mL}$ of $\mathrm{MeOH}$ in a hydrogenation flask. The heterogeneous reaction mixture was carefully purged with Ar-stream to replace dissolved $\mathrm{O}_{2}$ by Ar followed by purging with $\mathrm{H}_{2}$-baloon. Thereafter the flask was placed in the Parr's apparatus under 3-4 Bar $\mathrm{H}_{2}$ and shaken overnight at room temperature. After the hydrogenolysis was completed (indicated by TLC), the mixture was diluted with $30 \mathrm{~mL}$ of DCM and added $\mathrm{Boc}_{2} \mathrm{O}$ (1.5 eq.) or Fmoc-Osu (1.5 eq.) with DIPEA (2 eq.). The reaction mixture was stirred overnight at room temperature and on completion (checked on TLC) filtered through a pad of Celite and Florisil to remove remaining $\mathrm{Pd} / \mathrm{C}$ followed by washing the Celite and Florisil pad extensively with $\mathrm{MeOH}$. The collected fractions of filtrates were concentrated under reduced pressure. The crude product was purified by flash column chromatography with EtOAc/n-Hexane (1:10) to yield the purified $\mathrm{N}(\mathrm{Boc}) /(\mathrm{Fmoc})$-protected $\beta$-substituted $\gamma$-amino alcohol, tert-butyl (S)-(2 (hydroxymethyl)-4-methylpentyl)carbamate or $\quad$ (9H-fluoren-9-yl)methyl (S)-(2(hydroxymethyl)-4-methylpentyl)carbamate with 48\% and 56\% yields, respctively.

1H NMR (300 MHz, CDCl3, Boc protected): $\delta$ 7.34-7.26 (m, 10H), $5.12(\mathrm{br}, 1 \mathrm{H}), 4.03(\mathrm{q}, J=6.9$ $\mathrm{Hz}, 1 \mathrm{H}), 3.98 \& 3.93(\mathrm{~s}, 1 \mathrm{H}), 3.54(\mathrm{br}, 1 \mathrm{H}), 3.39 \& 3.35$ (s, 1H), 2.98-2.92 (m, 1H), 2.56-2.52 (m, $1 \mathrm{H}), 2.46-2.38(\mathrm{~m}, 1 \mathrm{H}), 2.08-1.93(\mathrm{br}, 1 \mathrm{H}), 1.58-1.54(\mathrm{~m}, 1 \mathrm{H}), 1.35(\mathrm{~d}, \mathrm{~J}=6.9 \mathrm{~Hz}, 3 \mathrm{H}), 0.90-0.78$ $(\mathrm{m}, 6 \mathrm{H})$.

${ }^{13}$ C NMR (75 MHz, CDCl3, Boc protected): $\delta$ 142.8, 138.9, 129.5, 128.7, 128.4, 128.3, 127.5, $127.3,68.8,56.5,55.3,55.1,39.5,34.5,25.6,23.1,22.9,9.5$.

ESI-MS (Boc protected): $(\mathrm{m} / \mathrm{z}): 232.4[\mathrm{M}+\mathrm{H}]^{+}, 254.3[\mathrm{M}+\mathrm{Na}]^{+}, 270.3[\mathrm{M}+\mathrm{K}]^{+}$

HRMS (ESI, Boc protected): calc. for $\left[\mathrm{C}_{12} \mathrm{H}_{26} \mathrm{NO}_{3}\right]^{+}\left([\mathrm{M}+\mathrm{H}]^{+}\right): 232.3933$, found: 232.4047; calc. for $\left[\mathrm{C}_{12} \mathrm{H}_{25} \mathrm{NO}_{3} \mathrm{Na}\right]^{+}\left([\mathrm{M}+\mathrm{Na}]^{+}\right): 254.3677$, found: 254.3684 


\subsubsection{8 (S)-2-(((tert-butoxycarbonyl)amino)methyl)-3-methylbutanoic acid}

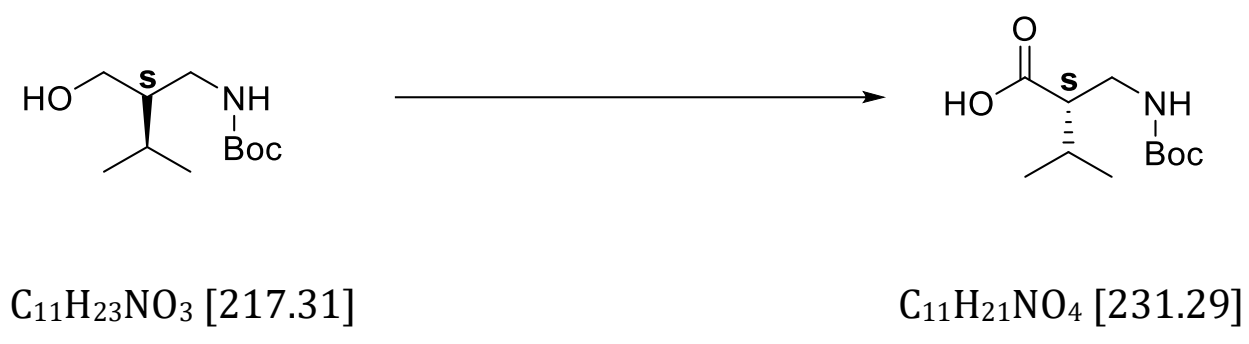

Synthetic procedure: In order to prepare fresh Jones reagent $50 \mathrm{mmol} \mathrm{Na}_{2} \mathrm{Cr}_{2} \mathrm{O}_{7}$ was dissolved in $50 \mathrm{~mL}$ of $\mathrm{H}_{2} \mathrm{O}$ and was slowly added $200 \mathrm{~mol}$ concentrated $\mathrm{H}_{2} \mathrm{SO}_{4}$. The solution was then diluted to $100 \mathrm{~mL}$ to give the Jones reagent $\left(0.5 \mathrm{M} \mathrm{H}_{2} \mathrm{Cr}_{2} \mathrm{O}_{7}\right)$. Now the freshly prepared $\mathrm{H}_{2} \mathrm{Cr}_{2} \mathrm{O}_{7}$ (Jones reagent, 1.5 eq., $8.8 \mathrm{mmol}$ ) was added to a solution of $\mathrm{N}(\mathrm{Boc})$-protected $\beta$-substituted $\gamma$ amino alcohol, tert-butyl (S)-(2-(hydroxymethyl)-3-methylbutyl)carbamate (1 eq., 1.3 g, 5.9 mmol) in $15 \mathrm{~mL}$ acetone in $0^{\circ} \mathrm{C}$. The mixture was stirred $10 \mathrm{hrs}$ and the temperature was gradually reduced from $0^{\circ} \mathrm{C}$ to room temperature. Then excess isopropanol was added and the mixture was kept for stirring overnight. Afterwards the turbid reaction mixture was filtered and the filtrate was concentrated under reduced pressure until $6 \mathrm{~mL}$ and further diluted with $4 \mathrm{~mL}$ of $2 \mathrm{~N}$ aqueous HCL and extracted with $\mathrm{Et}_{2} \mathrm{O}$ until TLC indicated that all the desired product had been removed from the aqueous phase to the organic phase. The combined $\mathrm{Et}_{2} \mathrm{O}$ fractions were collected and concentrated under reduced pressure until $20 \mathrm{~mL}$. Now the concentrated $\mathrm{Et}_{2} \mathrm{O}$ fraction was extracted with $2 \mathrm{M}$ aqueous $\mathrm{NaOH}$ solution. The combined alkaline aqueous fractions were washed with $\mathrm{Et}_{2} \mathrm{O}(2 \times 10 \mathrm{~mL})$ and the organic layers were discarded. The aqueous alkaline fraction was further acidified with $2 \mathrm{~N}$ aqueous $\mathrm{HCL}$ and extracted with $\mathrm{Et}_{2} \mathrm{O}$ until the desired product was completed moved to the organic phase (monitored by TLC). The combined $\mathrm{Et}_{2} \mathrm{O}$ layers were washed thoroughly with saturated brine solution and saturated $\mathrm{NH}_{4} \mathrm{Cl}$ solution. The organic fraction was dried over anhydrous $\mathrm{Na}_{2} \mathrm{SO}_{4}$ pad and filtered. The filtrate was concentrated under reduced pressure to give crude N(Boc)- $\beta^{2}$-homo valine, (S)-2(((tert-butoxycarbonyl)amino)methyl)-3-methylbutanoic acid that was further purified by flash column chromatography DCM/MeOH (12:1 to 12:3 in linear gradient) to yield the pure product (35\% yield).

1H NMR (300 MHz, CDCl3): $\delta 6.90 \& 5.05$ (br, 1H), 3.53-3.38 (m, 1H), 3.29-3.10 (m, 1H), 2.50$2.41(\mathrm{~m}, 1 \mathrm{H}), 2.05-1.89(\mathrm{~m}, 1 \mathrm{H}), 1.49-1.45(\mathrm{~m}, 9 \mathrm{H}), 1.02-0.96(\mathrm{~m}, 6 \mathrm{H})$. 
${ }^{13}$ C NMR (75 MHz, CDCl3): $\delta 180.6$ \& 179.3, $158.1 \& 156.2,81.2$ \& 79.8, 45.9 \& 45.6, 42.9 \& 41.6, $31.9,28.6,20.4,14.1$.

ESI-MS: $(\mathrm{m} / \mathrm{z}):$ 230.2[M-H]- $461.3\left[2 \mathrm{M}-\mathrm{H}^{-}\right.$

HRMS (ESI): calc. for $\left[\mathrm{C}_{11} \mathrm{H}_{20} \mathrm{NO}_{4}\right]^{-}\left([\mathrm{M}-\mathrm{H}]^{-}\right): 230.3011$, found: 230.3024

\subsubsection{9 (S)-2-(((tert-butoxycarbonyl)amino)methyl)-4-methylpentanoic acid}
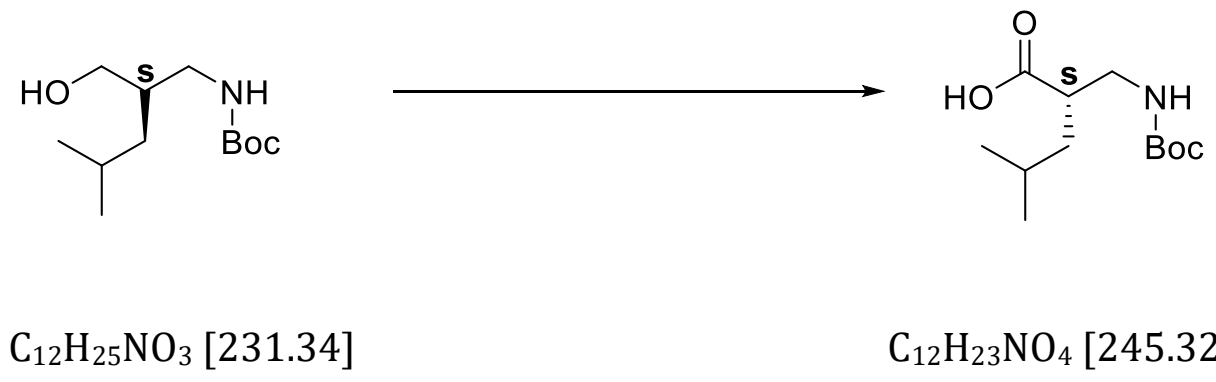

$\mathrm{C}_{12} \mathrm{H}_{23} \mathrm{NO}_{4}[245.32]$

Sythetic procedure: Synthetic procedure: In order to prepare fresh Jones reagent $50 \mathrm{mmol}$ $\mathrm{Na}_{2} \mathrm{Cr}_{2} \mathrm{O}_{7}$ was dissolved in $50 \mathrm{~mL}$ of $\mathrm{H}_{2} \mathrm{O}$ and was slowly added 200 mol concentrated $\mathrm{H}_{2} \mathrm{SO}_{4}$. The solution was then diluted to $100 \mathrm{~mL}$ to give the Jones reagent ( $0.5 \mathrm{M} \mathrm{H}_{2} \mathrm{Cr}_{2} \mathrm{O}_{7}$ ). Now the freshly prepared $\mathrm{H}_{2} \mathrm{Cr}_{2} \mathrm{O}_{7}$ (Jones reagent, 1.5 eq., $7.5 \mathrm{mmol}$ ) was added to a solution of $\mathrm{N}(\mathrm{Boc})$-protected $\beta$-substituted $\gamma$-amino alcohol, tert-butyl $(S)$-(2-(hydroxymethyl)-4-methylpentyl)carbamate (1 eq., $1.1 \mathrm{~g}, 4.7 \mathrm{mmol}$ ) in $13 \mathrm{~mL}$ acetone in $0^{\circ} \mathrm{C}$. The mixture was stirred $14 \mathrm{hrs}$ and the temperature was gradually reduced from $0^{\circ} \mathrm{C}$ to room temperature. Then excess isopropanol was added and the mixture was kept for stirring overnight. Afterwards the turbid reaction mixture was filtered and the filtrate was concentrated under reduced pressure until $5 \mathrm{~mL}$ and further diluted with $3 \mathrm{~mL}$ of $2 \mathrm{~N}$ aqueous HCL and extracted with $\mathrm{Et}_{2} \mathrm{O}$ until TLC indicated that all the desired product had been removed from the aqueous phase to the organic phase. The combined $\mathrm{Et}_{2} \mathrm{O}$ fractions were collected and concentrated under reduced pressure until $20 \mathrm{~mL}$. Now the concentrated $\mathrm{Et}_{2} \mathrm{O}$ fraction was extracted with $2 \mathrm{M}$ aqueous $\mathrm{NaOH}$ solution. The combined alkaline aqueous fractions were washed with $\mathrm{Et}_{2} \mathrm{O}(2 \times 10 \mathrm{~mL})$ and the organic layers were discarded. The aqueous alkaline fraction was further acidified with $2 \mathrm{~N}$ aqueous HCL and extracted with $\mathrm{Et}_{2} \mathrm{O}$ until the desired product was completed moved to the organic phase 
(monitored by TLC). The combined $\mathrm{Et}_{2} \mathrm{O}$ layers were washed thoroughly with saturated brine solution and saturated $\mathrm{NH}_{4} \mathrm{Cl}$ solution. The organic fraction was dried over anhydrous $\mathrm{Na}_{2} \mathrm{SO}_{4}$ pad and filtered. The filtrate was concentrated under reduced pressure to give crude N(Boc)- $\beta^{2}$ homo leucine, (S)-2-(((tert-butoxycarbonyl)amino)methyl)-4-methylpentanoic acid, that was further purified by flash column chromatography DCM/MeOH (10:1 to 10:3 in linear gradient) to yield the pure product (29\% yield).

${ }^{1}$ H NMR (300 MHz, CDCl3): $\delta 10.26$ (br, 1H), $6.68 \& 5.04(\mathrm{br}, 1 \mathrm{H}), 3.44-2.98(\mathrm{~m}, 2 \mathrm{H}), 2.73-2.61$ $(\mathrm{m}, 1 \mathrm{H}), 1.75-1.65(\mathrm{~m}, 1 \mathrm{H}), 1.54-1.44(\mathrm{~m}, 11 \mathrm{H}), 0.94-0.91(\mathrm{~m}, 6 \mathrm{H})$.

${ }^{13}$ C NMR (75 MHz, CDCl3): $\delta 181.0 \& 179.6,158.1 \& 156.1,81.3 \&$ 79.8, $44.3 \&$ 44.0, $43.3 \&$ 42.0, $39.1 \& 38.8,29.9,20.5,26.0,22.60 \& 22.56$.

ESI-MS: $(\mathrm{m} / \mathrm{z}):$ 244.3[M-H]-, 489.3[2M-H] ${ }^{-}$

HRMS (ESI): calc. for [ $\left.\mathrm{C}_{12} \mathrm{H}_{22} \mathrm{NO}_{4}\right]^{-}\left([\mathrm{M}-\mathrm{H}]^{-}\right): 244.3266$, found: 244.3275

\subsubsection{0 (S)-2-((((9H-fluoren-9-yl)methoxy)carbonyl)amino)methyl)-3-methylbutanoic acid}<smiles>CC(C)[C@@H](CO)CNPCOC(=O)O</smiles>

$\mathrm{C}_{21} \mathrm{H}_{25} \mathrm{NO}_{3}[339.44]$

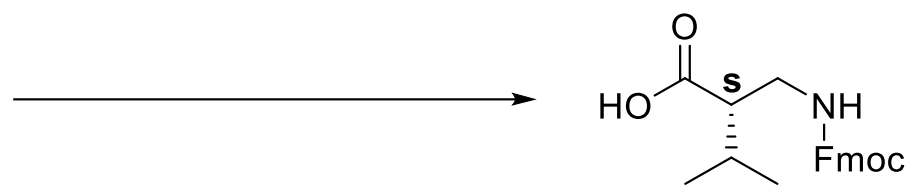

$\mathrm{C}_{21} \mathrm{H}_{23} \mathrm{NO}_{4}[353.42]$

Synthetic procedure: In order to prepare fresh Jones reagent $50 \mathrm{mmol} \mathrm{Na}_{2} \mathrm{Cr}_{2} \mathrm{O}_{7}$ was dissolved in $50 \mathrm{~mL}$ of $\mathrm{H}_{2} \mathrm{O}$ and was slowly added 200 mol concentrated $\mathrm{H}_{2} \mathrm{SO}_{4}$. The solution was then diluted to $100 \mathrm{~mL}$ to give the Jones reagent $\left(0.5 \mathrm{M} \mathrm{H}_{2} \mathrm{Cr}_{2} \mathrm{O}_{7}\right.$ ). Now the freshly prepared $\mathrm{H}_{2} \mathrm{Cr}_{2} \mathrm{O}_{7}$ (Jones reagent, 1.5 eq., $6.1 \mathrm{mmol}$ ) was added to a solution of N(Fmoc)-protected $\beta$-substituted $\gamma$ amino alcohol, (9H-fluoren-9-yl)methyl (S)-(2-(hydroxymethyl)-3-methylbutyl)carbamate (1 eq., $1.4 \mathrm{~g}, 4.1 \mathrm{mmol}$ ) in $15 \mathrm{~mL}$ acetone in $0^{\circ} \mathrm{C}$. The mixture was stirred $12 \mathrm{hrs}$ and the temperature was gradually reduced from $0^{\circ} \mathrm{C}$ to room temperature. Then excess isopropanol 
was added and the mixture was kept for stirring overnight. Afterwards the turbid reaction mixture was filtered and the filtrate was concentrated under reduced pressure until $7 \mathrm{~mL}$ and further diluted with $5 \mathrm{~mL}$ of $2 \mathrm{~N}$ aqueous HCL and extracted with $\mathrm{Et}_{2} \mathrm{O}$ until TLC indicated that all the desired product had been removed from the aqueous phase to the organic phase. The combined $\mathrm{Et}_{2} \mathrm{O}$ fractions were collected and concentrated under reduced pressure until $25 \mathrm{~mL}$. Now the concentrated $\mathrm{Et}_{2} \mathrm{O}$ fraction was extracted with $2 \mathrm{M}$ aqueous $\mathrm{NaOH}$ solution. The combined alkaline aqueous fractions were washed with $\mathrm{Et}_{2} \mathrm{O}(2 \times 10 \mathrm{~mL})$ and the organic layers were discarded. The aqueous alkaline fraction was further acidified with $2 \mathrm{~N}$ aqueous HCL and extracted with $\mathrm{Et}_{2} \mathrm{O}$ until the desired product was completed moved to the organic phase (monitored by TLC). The combined $\mathrm{Et}_{2} \mathrm{O}$ layers were washed thoroughly with saturated brine solution and saturated $\mathrm{NH}_{4} \mathrm{Cl}$ solution. The organic fraction was dried over anhydrous $\mathrm{Na}_{2} \mathrm{SO}_{4}$ pad and filtered. The filtrate was concentrated under reduced pressure to give crude N(Fmoc)$\beta^{2}$-homo-valine,(S)-2-((c((9H-fluoren-9-yl)methoxy)carbonyl)amino)methyl)-3-methylbutanoic acid, that was further purified by flash column chromatography DCM/MeOH (10:1) to yield the pure product ( $37 \%$ yield).

${ }^{1}$ H NMR (300 MHz, CDCl3): $\delta=7.86-7.68(\mathrm{dd}, J=7.5,4.7,2 \mathrm{H}, \mathrm{CH}), 7.67-7.49(\mathrm{~m}, 2 \mathrm{H}, \mathrm{CH}), 7.45$ - $7.21(\mathrm{~m}, 4 \mathrm{H}, \mathrm{CH}), 7.10-6.94(\mathrm{dd}, \mathrm{J}=8.2,4.9,1 \mathrm{H}, \mathrm{NH}), 5.37-5.23\left(\mathrm{~m}, 2 \mathrm{H}, \mathrm{CH}_{2}\right), 4.64-4.50(\mathrm{~m}$, $1 \mathrm{H}, \mathrm{CH}), 3.63-2.96$ (m, 2H, CH2), $2.31-2.13$ (ddd, J=10.9, 6.3, 4.2, 1H, CH), 1.87 - 1.65 (h, J=6.8, $1 \mathrm{H}, \mathrm{CH}), 1.16-0.79\left(\mathrm{~m}, 6 \mathrm{H}, \mathrm{CH}_{3}\right)$.

ESI-MS: $(\mathrm{m} / \mathrm{z}): 354.3[\mathrm{M}+\mathrm{H}]^{+}, 376.4[\mathrm{M}+\mathrm{Na}]^{+}, 707.4[2 \mathrm{M}+\mathrm{H}]^{+}$

HRMS (ESI): calc. for $\left[\mathrm{C}_{21} \mathrm{H}_{24} \mathrm{NO}_{4}\right]^{+}\left([\mathrm{M}+\mathrm{H}]^{+}\right)$: 354.3997, found: 354.3990; calc. for $\left[\mathrm{C}_{21} \mathrm{H}_{23} \mathrm{NO}_{4} \mathrm{Na}\right]^{+}\left([\mathrm{M}+\mathrm{Na}]^{+}\right): 376.4216$, found: 376.4209

\subsubsection{1(S)-2-(((((9H-fluoren-9-yl)methoxy)carbonyl)amino)methyl)-4-methylpentanoic acid}
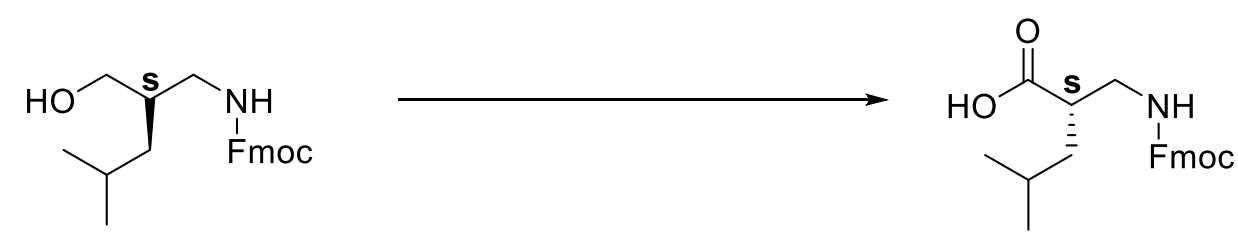


$$
\mathrm{C}_{22} \mathrm{H}_{27} \mathrm{NO}_{3}[353.46] \quad \mathrm{C}_{22} \mathrm{H}_{25} \mathrm{NO}_{4}[367.45]
$$

Synthetic procedure: In order to prepare fresh Jones reagent 50 mmol $\mathrm{Na}_{2} \mathrm{Cr}_{2} \mathrm{O}_{7}$ was dissolved in $50 \mathrm{~mL}$ of $\mathrm{H}_{2} \mathrm{O}$ and was slowly added 200 mol concentrated $\mathrm{H}_{2} \mathrm{SO}_{4}$. The solution was then diluted to $100 \mathrm{~mL}$ to give the Jones reagent $\left(0.5 \mathrm{M} \mathrm{H}_{2} \mathrm{Cr}_{2} \mathrm{O}_{7}\right.$ ). Now the freshly prepared $\mathrm{H}_{2} \mathrm{Cr}_{2} \mathrm{O}_{7}$ (Jones reagent, 1.5 eq., $5.09 \mathrm{mmol}$ ) was added to a solution of $\mathrm{N}(\mathrm{Fmoc})$-protected $\beta$-substituted $\gamma$-amino alcohol, (9H-fluoren-9-yl)methyl (S)-(2-(hydroxymethyl)-4-methylpentyl)carbamate (1 eq., $1.2 \mathrm{~g}, 3.3 \mathrm{mmol}$ ) in $12 \mathrm{~mL}$ acetone in $0^{\circ} \mathrm{C}$. The mixture was stirred $16 \mathrm{hrs}$ and the temperature was gradually reduced from $0^{\circ} \mathrm{C}$ to room temperature. Then excess isopropanol was added and the mixture was kept for stirring overnight. Afterwards the turbid reaction mixture was filtered and the filtrate was concentrated under reduced pressure until $5 \mathrm{~mL}$ and further diluted with $4 \mathrm{~mL}$ of $2 \mathrm{~N}$ aqueous HCL and extracted with $\mathrm{Et}_{2} \mathrm{O}$ until TLC indicated that all the desired product had been removed from the aqueous phase to the organic phase. The combined $\mathrm{Et}_{2} \mathrm{O}$ fractions were collected and concentrated under reduced pressure until $20 \mathrm{~mL}$. Now the concentrated $\mathrm{Et}_{2} \mathrm{O}$ fraction was extracted with $2 \mathrm{M}$ aqueous $\mathrm{NaOH}$ solution. The combined alkaline aqueous fractions were washed with $\mathrm{Et}_{2} \mathrm{O}(2 \times 10 \mathrm{~mL})$ and the organic layers were discarded. The aqueous alkaline fraction was further acidified with $2 \mathrm{~N}$ aqueous HCL and extracted with $\mathrm{Et}_{2} \mathrm{O}$ until the desired product was completed moved to the organic phase (monitored by TLC). The combined $\mathrm{Et}_{2} \mathrm{O}$ layers were washed thoroughly with saturated brine solution and saturated $\mathrm{NH}_{4} \mathrm{Cl}$ solution. The organic fraction was dried over anhydrous $\mathrm{Na}_{2} \mathrm{SO}_{4}$ pad and filtered. The filtrate was concentrated under reduced pressure to give crude N(Fmoc)$\beta^{2}$-homoleucine,(S)-2-((c((9H-fluoren-9-yl)methoxy)carbonyl)amino)methyl)-4methylpentanoic acid, that was further purified by flash column chromatography DCM/MeOH (10:1 to 10:4 in linear gradient) to yield the pure product (32\% yield).

${ }^{1} \mathrm{H}$ NMR (300 MHz, CDCl3): $\delta=7.84-7.64(\mathrm{dd}, J=7.5,4.7,2 \mathrm{H}, \mathrm{CH}), 7.66-7.47(\mathrm{~m}, 2 \mathrm{H}, \mathrm{CH}), 7.44-$ $7.22(\mathrm{~m}, 4 \mathrm{H}, \mathrm{CH}), 7.12-6.92(\mathrm{dd}, \mathrm{J}=8.2,4.9,1 \mathrm{H}, \mathrm{NH}), 5.35-5.21\left(\mathrm{~m}, 2 \mathrm{H}, \mathrm{CH}_{2}\right), 4.63-4.50(\mathrm{~m}, 1 \mathrm{H}$, $\mathrm{CH}$ ), $3.62-2.97\left(\mathrm{~m}, 2 \mathrm{H}, \mathrm{CH}_{2}\right.$ ), $2.30-2.11$ (ddd, J=10.9, 6.3, 4.2, 1H, CH), $1.85-1.65$ (h, J=6.8, $1 \mathrm{H}$, $\mathrm{CH}), 1.14-0.79\left(\mathrm{~m}, 6 \mathrm{H}, \mathrm{CH}_{3}\right)$. 


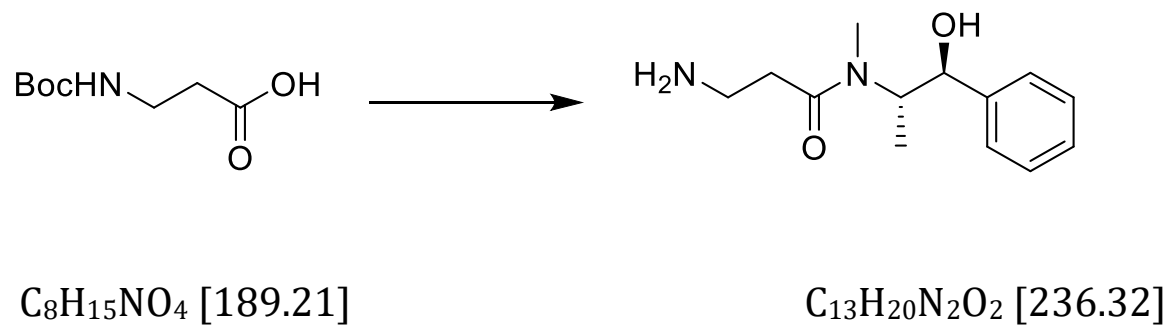

Synthetic Procedure: Pivaloyl chloride (1 eq., $7.8 \mathrm{~mL}, 63.4 \mathrm{mmol}$ ) was added to an ice-cold solution of Boc- $\beta$-alanine (1 eq., $12.0 \mathrm{~g}, 63.4 \mathrm{mmol}$ ) and triethylamine (1.2 eq., $10.6 \mathrm{~mL}, 76.1$ $\mathrm{mmol})$ in anhydrous dichloromethane $(160 \mathrm{~mL})$. The resulting mixture was stirred at $0{ }^{\circ} \mathrm{C}$ for 1 h. Additional triethylamine (1.2 eq., $10.6 \mathrm{~mL}, 76.1 \mathrm{mmol})$ was added to the reaction mixture and after 5 minutes a solution of (1S,2S)-(+)-pseudoephedrine (1 eq., $10.47 \mathrm{~g}, 63.4 \mathrm{mmol}$ ) dissolved in dichloromethane $(20 \mathrm{~mL})$ was added. The resulting mixture was warmed to room temperature and was stirred overnight (18 hrs) and then the excess solvent was evaporated under reduced pressure yielding viscous residue. The residue was further dissolved in 50\% aqueous methanol $(50 \mathrm{~mL})$ and cooled to $0{ }^{\circ} \mathrm{C}$ followed by the addition of concentrated aqueous $\mathrm{HCl}(45 \mathrm{~mL})$. The resulting solution was stirred for $5 \mathrm{~h}$ and then dried under reduced pressure. The resulting residue was dissolved in water and the aqueous solution was washed with $50 \%$ ethyl acetate/hexane and then made alkaline with $50 \%$ aqueous $\mathrm{NaOH}(\mathrm{pH}>12)$. The resulting basic aqueous mixture was extracted with dichloromethane $(5 \times 70 \mathrm{~mL})$. The combined organic (dichloromethane) fractions were dried over $\mathrm{Na}_{2} \mathrm{SO}_{4}$ and then filtered giving a turbid mixture. This mixture was again dried over $\mathrm{K}_{2} \mathrm{CO}_{3}$ (overnight) and then filtered through a pad of celite. The solvent was removed under reduced pressure giving rise to a colorless viscous oil. The viscous oil was recrystallized from hot toluene to give the desired compound as a white solid.

${ }^{1} \mathrm{H}$ NMR (300 MHz, $*$ denotes peaks due to minor rotamer, $\left.\mathrm{CDCl} 3\right): \delta=0.971 *(\mathrm{~d}, 1 \mathrm{H}, J=6.5$ $\mathrm{Hz}), 1.04(\mathrm{~d}, 2 \mathrm{H}, J=6.5 \mathrm{~Hz}), 2.36-2.54(\mathrm{~m}, 2 \mathrm{H}), 2.62(\mathrm{br}-\mathrm{s}, 2 \mathrm{H}), 2.81-2.86^{*}(\mathrm{~m}, 0.7 \mathrm{H}), 2.86(\mathrm{~s}, 2 \mathrm{H})$, $2.93^{*}(\mathrm{~s}, 1 \mathrm{H}), 2.98-3.30(\mathrm{~m}, 1.3 \mathrm{H}), 4.04-4.15^{*}(\mathrm{~m}, 0.3 \mathrm{H}), 4.51-4.65(\mathrm{~m}, 1.7 \mathrm{H}), 7.26-7.38(\mathrm{~m}, 5 \mathrm{H})$.

${ }^{13} \mathrm{C}$ NMR (75 MHz, * denotes peaks due to minor rotamer, $\mathbf{C D C l}$ ): $\delta=14.36,15.51 *, 26.73$, $36.02^{*}, 36,85,37.764,38.03^{*}, 56.31 *$, 58.23, 74.94*, 75.59, 126.9, 126.8*, 127.4*, 127.7, 128.3*, $128.6,142.0^{*}, 142.7,173.4^{*}, 173.8$.

ESI-MS: $(\mathrm{m} / \mathrm{z}): 237.3[\mathrm{M}+\mathrm{H}]^{+}, 259.3[\mathrm{M}+\mathrm{Na}]^{+}, 473.6[2 \mathrm{M}+\mathrm{H}]^{+}, 709.7[3 \mathrm{M}+\mathrm{H}]^{+}$ 
HRMS (ESI): calc. for $\left[\mathrm{C}_{13} \mathrm{H}_{21} \mathrm{~N}_{2} \mathrm{O}_{2}\right]^{+}\left([\mathrm{M}+\mathrm{H}]^{+}\right)$: 237.3278, found: 237.3271; calc. for $\left[\mathrm{C}_{13} \mathrm{H}_{20} \mathrm{~N}_{2} \mathrm{O}_{2} \mathrm{Na}\right]^{+}\left([\mathrm{M}+\mathrm{Na}]^{+}\right): 259.3155$, found: 259.3148

\subsubsection{3(S)-3-amino-N-((1S,2S)-1-hydroxy-1-phenylpropan-2-yl)-N,2-dimethylpropanamide}

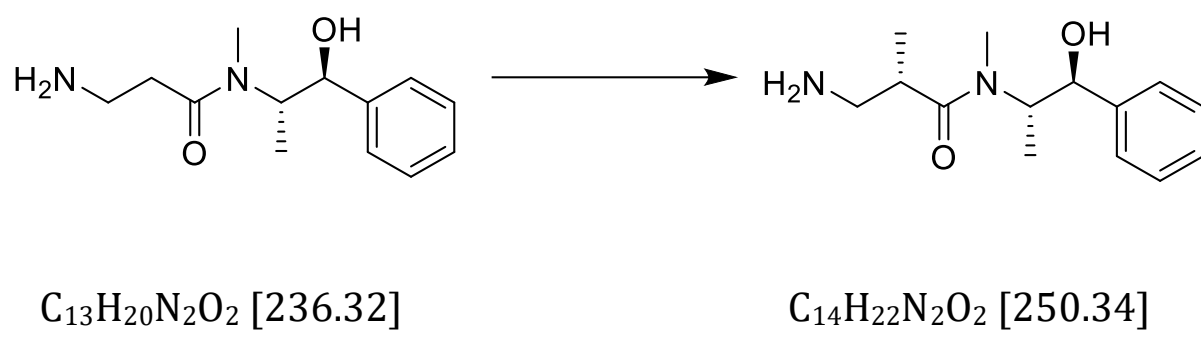

Synthetic procedure: Pseudoephedrine amide (1 eq., $2.0 \mathrm{~g}, 8.4 \mathrm{mmol}$ ) was dissolved in anhydrous THF (approximately $20 \mathrm{~mL} / \mathrm{g}$ ) and flame dried LiCl (6 eq., $2.15 \mathrm{~g}, 50.7 \mathrm{mmol}$ ) was added to it. The mixture was cooled to $-5{ }^{\circ} \mathrm{C}$ cryostat and lithium bis(trimethylsilyl)amide (3.2 eq., $5.22 \mathrm{~mL}, 26.88 \mathrm{mmol}$ ) was slowly added to the cold solution. Iodomethane (1.5 eq., $0.78 \mathrm{~mL}$, $12.6 \mathrm{mmol}$ ) was added to the reaction mixture after $1 \mathrm{~h}$ stirring, followed by stirring for $4 \mathrm{~h}$ until no starting material was observed by TLC. Double distilled water was added to the reaction mixture followed by acidification with $6 \mathrm{~N} \mathrm{HCl}$ to $\mathrm{pH} \sim 3$. The resulting mixture was washed with $50 \%$ ethyl acetate/hexane, and the aqueous fraction was cooled to $0^{\circ} \mathrm{C}$ in an ice-bath. The aqueous phase was further basified with $50 \%$ aqueous $\mathrm{NaOH}$ to $\mathrm{pH}>12$, and extracted with dichloromethane (4x50 mL). The combined organic fractions (dichloromethane) were dried over $\mathrm{Na}_{2} \mathrm{SO}_{4}$, filtered and concentrated under reduced pressure yielding the desired pseudoephedrine- $\beta$-alanine amide derivative, $(S)$-3-amino-N-((1S,2S)-1-hydroxy-1phenylpropan-2-yl)-N,2-dimethylpropanamide. The crude product was recrystallized from ether to give rise to the pure product as a colourless oil with $75 \%$ yield.

${ }^{1} \mathrm{H}$ NMR (300 MHz, * denotes peaks due to minor rotamer, $\left.\mathbf{C D C l}_{3}\right): \delta=0.961-1.07(\mathrm{~m}, 5.7 \mathrm{H})$, 1.19* (d, 0.3H, $J=7.0 \mathrm{~Hz}), 2.41^{*}(\mathrm{~s}, 0.2 \mathrm{H}), 2.68-2.97(\mathrm{~m}, 9 \mathrm{H}), 4.53(\mathrm{~d}, 0.9 \mathrm{H}$, $J=9.0 \mathrm{~Hz}), 4.58^{*}(\mathrm{~d}, 0.1 \mathrm{H}, J=9.0 \mathrm{~Hz}), 4.75(\mathrm{br}-\mathrm{s}, 1 \mathrm{H}), 7.26-7.37(\mathrm{~m}, 5 \mathrm{H})$. 
${ }^{13} \mathrm{C}$ NMR (75 MHz, * denotes peaks due to minor rotamer, $\mathbf{C D C l}_{3}$ ): $\delta=14.30 * 14.90,15.22$, 15.39*, 39.03, 39.09*, 45.83, 45.94*, 57.99, 65.74, 74.78*, 75.37, 126.5, 126.7*, 127.6, 128.2, $128.5^{*}, 142.8,176.5$.

ESI-MS: $(m / z): 251.3[\mathrm{M}+\mathrm{H}]^{+}, 273.3[\mathrm{M}+\mathrm{Na}]^{+}, 289.4[\mathrm{M}+\mathrm{K}]^{+} ; 501.7[2 \mathrm{M}+\mathrm{H}]^{+}$

HRMS (ESI): calc. for $\left[\mathrm{C}_{14} \mathrm{H}_{23} \mathrm{~N}_{2} \mathrm{O}_{2}\right]^{+}\left([\mathrm{M}+\mathrm{H}]^{+}\right): 251.3477$, found: 251.3473; calc. for $\left[\mathrm{C}_{14} \mathrm{H}_{22} \mathrm{~N}_{2} \mathrm{O}_{2} \mathrm{Na}\right]^{+}\left([\mathrm{M}+\mathrm{Na}]^{+}\right): 273.3799$, found: 273.3787

\subsubsection{4 (S)-3-amino-2-methylpropanoic acid}

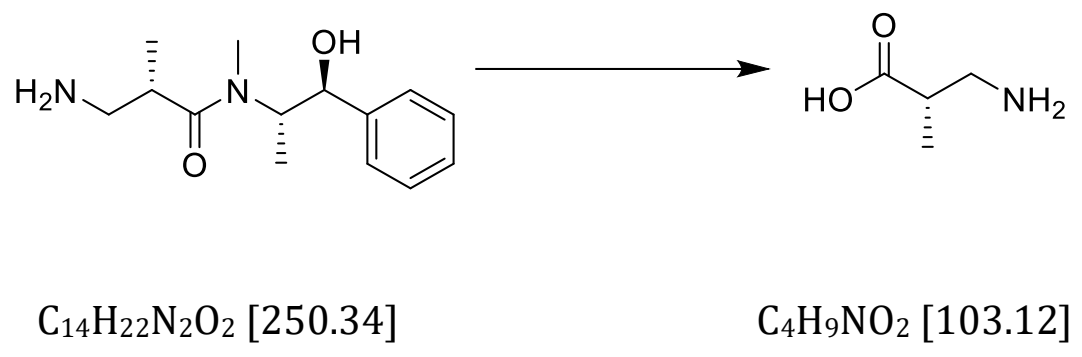

Synthetic procedure: Water $(\sim 10 \mathrm{~mL} / \mathrm{g})$ was added to the pseudoephedrine amide derivative, (S)-3-amino-N-((1S,2S)-1-hydroxy-1-phenylpropan-2-yl)-N,2-dimethylpropanamide, and the aqueous mixture was heated to reflux and stirred for $72 \mathrm{~h}$. Afterwards, the reaction mixture was cooled to room temperature and washed with dichloromethane $(3 \times 40 \mathrm{~mL})$. The aqueous solution was concentrated under reduce pressure or lyophilized (freeze-drying) yielding a solid that was recrystalized from ethanol to give the desired compound as a white solid with $82 \%$ yield.

${ }^{1} \mathbf{H}$ NMR (300 MHz, D 0$): ~ \delta=1.17(\mathrm{~d}, 3 \mathrm{H}, J=7.5 \mathrm{~Hz}), 2.54-2.62(\mathrm{~m}, 1 \mathrm{H}), 3.06\left(\mathrm{dd}, 1 \mathrm{H}, J_{1}=8.5 \mathrm{~Hz}\right.$, $\left.J_{2}=5.5 \mathrm{~Hz}\right), 3.11\left(\mathrm{dd}, 1 \mathrm{H}, J_{1}=13.0 \mathrm{~Hz}, J_{2}=8.5 \mathrm{~Hz}\right)$.

${ }^{13}$ C NMR (75 MHz, D $\mathbf{2}$ ): $\delta=19.76,43.92,46.96,186.6$.

ESI-MS: $(\mathrm{m} / \mathrm{z}): 104.1[\mathrm{M}+\mathrm{H}]^{+}, 126.1[\mathrm{M}+\mathrm{Na}]^{+} ; 207.2[2 \mathrm{M}+\mathrm{H}]^{+}$ 
HRMS (ESI): calc. for $\left[\mathrm{C}_{4} \mathrm{H}_{10} \mathrm{NO}_{2}\right]^{+}\left([\mathrm{M}+\mathrm{H}]^{+}\right): 104.1288$, found:104.1283; calc. for $\left[\mathrm{C}_{4} \mathrm{H}_{9} \mathrm{NO}_{2} \mathrm{Na}\right]^{+}$ $\left([\mathrm{M}+\mathrm{Na}]^{+}\right): 126.1239$, found: 126.1228

\subsubsection{Synthesis of $\boldsymbol{\beta}$-peptides}

\subsubsection{Synthesis of $\beta^{3}$-peptides}

8.4.2.1.1 HO- $\beta^{3 \mathrm{~h}} \mathrm{~V}-\beta^{3 \mathrm{~h}} \mathrm{~W}-\left(\beta^{3 \mathrm{~h}} \mathrm{~A}-\beta^{3 \mathrm{~h}} \mathrm{~L}-\beta^{3 \mathrm{~h}} \mathrm{~V}\right)_{3}-\beta^{3 \mathrm{~h}} \mathrm{~A}-\mathrm{NH}_{2}(\mathrm{P} 1)$

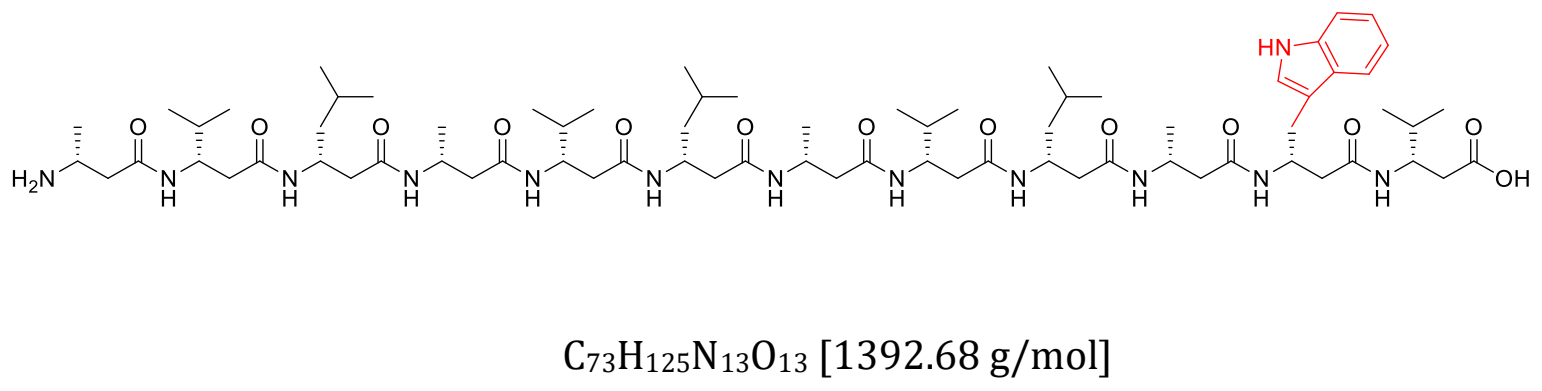

Synthesis: The 2-chlorotrityl chloride resin (1 eq., $122 \mathrm{mg}, 0.2 \mathrm{mmol}$ ) was pre-loaded with Fmoc-D- $\beta^{3}$-hVal-OH (3.0 eq., $212 \mathrm{mg}, 0.6 \mathrm{mmol}$ ) by the G.P - 7. The actual loading density was estimated to be $0.95 \mathrm{mmol} / \mathrm{g}$ following the method described in 6.4.2. The peptide was synthesized by performing a double coupling with Fmoc-D- $\beta^{3}$-hTrp(Boc)-OH (5.0 eq., 3.0 eq., 297 mg, $178 \mathrm{mg}, 0.55 \mathrm{mmol}, 0.33 \mathrm{mmol}$ ) using G.P - 8(1) via microwave-assisted SPPS. The rest of the amino acids, like, Fmoc-D- $\beta^{3}$ hAla-OH (3.0 eq., 107 mg, 0.33 mmol), Fmoc-D- $\beta^{3}$ hLeu-OH (3.0 eq., $121 \mathrm{mg}, 0.33 \mathrm{mmol}$ ) and Fmoc-D- $\beta^{3}$ hVal-OH (3.0 eq., $116 \mathrm{mg}, 0.33 \mathrm{mmol}$ ), were coupled using G.P - 9 via non-microwave assisted manual SPPS. The capping of the unreacted free amines were carried out following G.P - 6. The coupling was qualitatively checked by Kaiser Test as described in G.P - 10 after each coupling. After the final Fmoc-deprotection the resin was washed with a mixture of DCM/DIPEA/MeOH $(5 \times 2.0 \mathrm{ml})$, DMF $(5 \times 2.0 \mathrm{~mL})$ and DCM $(5 \times 2.0 \mathrm{~mL})$. Furthermore, the resin was dried overnight under reduced pressure. Next day, The cleavage was carried out via G.P - 12 to obtain the crude peptide as a white solid that was purified by semipreparative HPLC to yield desired pure peptide as a colourless solid. 
HPLC: (Column: Semi-preparative MN Nucleodur ${ }^{\circledR}$ 100-5-C18, 250mm $\times 10 \mathrm{~mm}, 5 \mu \mathrm{m}$, flow rate: $3 \mathrm{~mL} / \mathrm{min}$; Gradient: $20-90 \%$ B in $48 \mathrm{~min} ; \boldsymbol{\lambda}: 215,254,280 \mathrm{~nm}$ ); $\mathbf{t}_{\mathbf{R}}=21.6 \mathrm{~min}$

ESI-MS (m/z): $349.2[\mathrm{M}+4 \mathrm{H}]^{4+}, 465.2[\mathrm{M}+3 \mathrm{H}]^{3+}, 697.3[\mathrm{M}+2 \mathrm{H}]^{2+}, 1393.6[\mathrm{M}+\mathrm{H}]^{+}$

HR-MS (m/z): Calculated for $[\mathrm{M}+2 \mathrm{H}]^{2+}$ : 697.3055, found: 697.3039; Calculated for $[\mathrm{M}+3 \mathrm{H}]^{3+}$ : 465.2874, found: 465.2867; Calculated for $[\mathrm{M}+4 \mathrm{H}]^{4+}: 349.2963$ found: 349.2970

8.4.2.1.2 HO- $\left(\beta^{3 h^{h}}\right)_{2}-\left(\beta^{3 h} W\right)_{2}-\left(\beta^{3 h} V\right)_{19}-\left(\beta^{3 h} W\right)_{2}-\left(\beta^{3{ }^{h}} K\right)_{2}-N_{2}(P 4)$

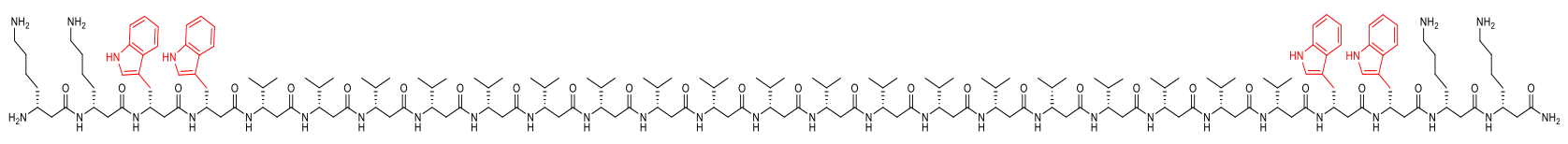

$\mathrm{C}_{190} \mathrm{H}_{316} \mathrm{~N}_{36} \mathrm{O}_{27}[3536.84 \mathrm{~g} / \mathrm{mmol}]$

Synthesis: The rink amide MBHA resin (1 eq., $132 \mathrm{mg}, 0.07 \mathrm{mmol}$ ) was loaded with Fmoc-D- $\beta^{3}$ hLys(Boc)-OH (5 eq., $180 \mathrm{mg}, 0.37 \mathrm{mmol}$ ) by the G.P - 5. The actual loading density was estimated to be $0.51 \mathrm{mmol} / \mathrm{g}$ following the method described in 6.4.2. The peptide was synthesized by performing double coupling with Fmoc-D- $\beta^{3-h T r p(B o c)-O H ~(5.0 ~ e q ., ~} 3.0$ eq., 202 mg, 118 mg, 0.37 mmol, 0.22 mmol), Fmoc-D- $\beta^{3-h}$ Lys(Boc)-OH (5 eq., 3.0 eq., 178 mg, 106 mg, 0.37 mmol, 0.22 mmol), Fmoc-D- $\beta^{3}$-hVal-OH (5 eq., 3.0 eq., 130 mg, 99.7 mg, 0.37 mmol, 0.22 mmol) using G.P 8(1) via microwave-assisted SPPS. The capping of the unreacted free amines were carried out following G.P - 6. The coupling was qualitatively checked by Kaiser Test as described in G.P - 10 after each coupling. After the final Fmoc-deprotection the resin was washed with a mixture of DCM/DIPEA/MeOH $(5 \times 2.0 \mathrm{ml})$, DMF $(5 \times 2.0 \mathrm{~mL})$ and DCM $(5 \times 2.0 \mathrm{~mL})$. Furthermore, the resin was dried overnight under reduced pressure. Next day, The cleavage was carried out via G.P - 12 to obtain the crude peptide as a white solid that was purified by semi-preparative HPLC to yield desired pure peptide as a white solid.

HPLC: (Column: Semi-preparative MN Nucleodur ${ }^{\circledR}$ 100-5-C18, 250mm $\times 10 \mathrm{~mm}, 5 \mu \mathrm{m}$, flow rate: $3 \mathrm{~mL} / \mathrm{min}$; Gradient: $80-97 \%$ B in $55 \mathrm{~min} ; \boldsymbol{\lambda}: 215,254,280 \mathrm{~nm}$ ); $\mathbf{t}_{\mathbf{R}}=38.9 \mathrm{~min}$ 
HR-MS (m/z): Calculated for [M+2H] ${ }^{2+}:$ 1769.7044, found: 1769.7036; Calculated for $[\mathrm{M}+3 \mathrm{H}]^{3+}$ : 1179.8422, found: 1179.8415; Calculated for $[\mathrm{M}+4 \mathrm{H}]^{4+}: 885.2047$ found: 885.2042

\subsection{HO- $\left(\beta^{3 h^{h}} K\right)_{1}-\left(\beta^{3 h} W\right)_{1}-\left(\beta^{3 h} V\right)_{12}-\left(\beta^{3 h} W\right)_{1}-\left(\beta^{3 h} K\right)_{1}-N^{2} H_{2}(P 10)$}

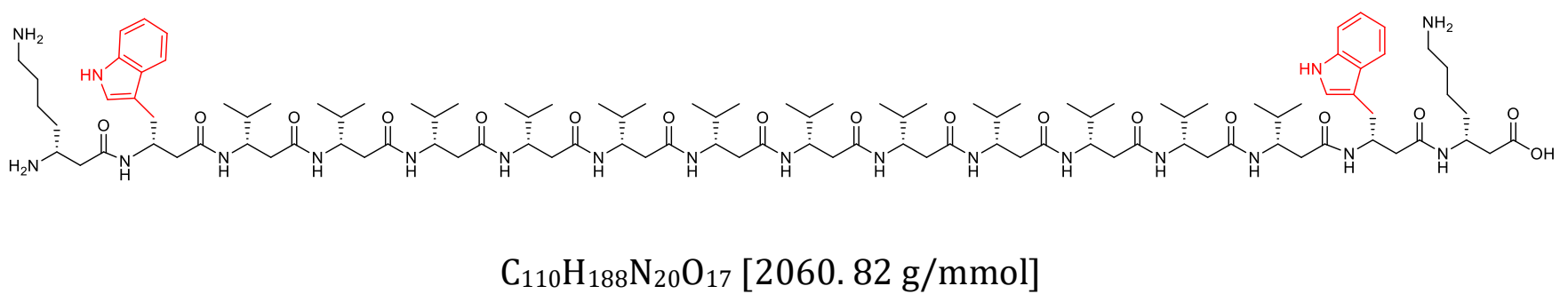

Synthesis: The rink amide MBHA resin (1 eq., $132 \mathrm{mg}, 0.07 \mathrm{mmol}$ ) was loaded with Fmoc-D- $\beta^{3}$ hLys(Boc)-OH (5 eq., $180 \mathrm{mg}, 0.37 \mathrm{mmol}$ ) by the G.P - 5. The actual loading density was estimated to be $0.51 \mathrm{mmol} / \mathrm{g}$ following the method described in 6.4.2. The peptide was synthesized by

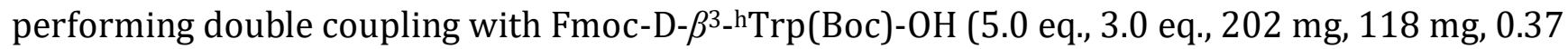
mmol, 0.22 mmol), Fmoc-D- $\beta^{3}$-hLys(Boc)-OH (5 eq., 3.0 eq., 178 mg, 106 mg, 0.37 mmol, 0.22 mmol), Fmoc-D- $\beta^{3}$-hVal-OH (5 eq., 3.0 eq., 130 mg, 99.7 mg, 0.37 mmol, 0.22 mmol) using G.P 8(1) via microwave-assisted SPPS. The capping of the unreacted free amines were carried out following G.P - 6. The coupling was qualitatively checked by Kaiser Test as described in G.P - 10 after each coupling. After the final Fmoc-deprotection the resin was washed with a mixture of DCM/DIPEA/MeOH $(5 \times 2.0 \mathrm{ml})$, DMF $(5 \times 2.0 \mathrm{~mL})$ and DCM $(5 \times 2.0 \mathrm{~mL})$. Furthermore, the resin was dried overnight under reduced pressure. Next day, The cleavage was carried out via G.P - 12 to obtain the crude peptide as a white solid that was purified by semi-preparative HPLC to yield desired pure peptide as a white solid.

HPLC: (Column: Semi-preparative MN Nucleodur ${ }^{\circledR}$ 100-5-C18, 250mm $\times 10 \mathrm{~mm}, 5 \mu \mathrm{m}$, flow rate: $3 \mathrm{~mL} / \mathrm{min}$; Gradient: $80-97 \%$ B in $55 \mathrm{~min}$; $\lambda: 215,254,280 \mathrm{~nm}$ ); $\mathbf{t}_{\mathbf{R}}=35.3 \mathrm{~min}$

ESI-MS (m/z): $515.2[\mathrm{M}+4 \mathrm{H}]^{4+}, 687.9[\mathrm{M}+3 \mathrm{H}]^{3+}, 1031.4[\mathrm{M}+2 \mathrm{H}]^{2+}$

HR-MS (m/z): Calculated for $[\mathrm{M}+2 \mathrm{H}]^{2+}:$ 1031.4022, found: 1031.4028 ; Calculated for $[\mathrm{M}+3 \mathrm{H}]^{3+}$ : 687.9168, found: 687.9174. 
8.4.2.1.4 HO- $\left(\beta^{3 h} K\right)_{2}-\left(\beta^{3 h} W\right)_{2}-\left(\beta^{3 h} L\right)_{15}-\left(\beta^{3 h} W\right)_{2}-\left(\beta^{3 h} K\right)_{2}-N_{2}(P 5)$

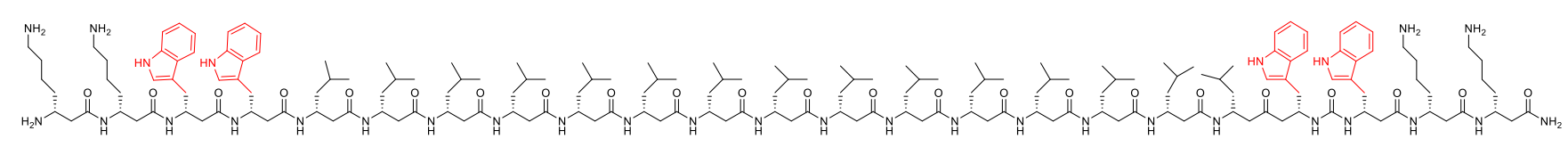

$\mathrm{C}_{181} \mathrm{H}_{302} \mathrm{~N}_{32} \mathrm{O}_{23}[3294.61 \mathrm{~g} / \mathrm{mmol}]$

Synthesis: The rink amide Nova PEG LL resin (1 eq., $1.1 \mathrm{~g}, 0.2 \mathrm{mmol}$ ) was loaded with Fmoc-D-

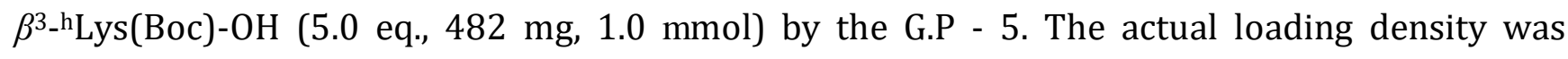
estimated to be $0.13 \mathrm{mmol} / \mathrm{g}$ following the method described in 6.4.2. The peptide was synthesized by performing double coupling with Fmoc-D- $\beta^{3}$-h Lys(Boc)-OH (5.0 eq. $(\times 2), 337 \mathrm{mg}$ $(\times 2), 0.7 \mathrm{mmol}(\times 2))$, Fmoc-D- $\beta^{3}$ h $\mathrm{Trp}($ Boc) $-\mathrm{OH}(5$ eq. $(\times 2), 378 \mathrm{mg}(\times 2), 0.7 \mathrm{mmol}(\times 2))$, FmocD- $\beta^{3}$-hLeu-OH (5 eq. $(\times 2), 257 \mathrm{mg}(\times 2), 0.7 \mathrm{mmol}(\times 2)$ ) using G.P - 8(2) via microwave-assisted SPPS. The capping of the unreacted free amines were carried out following G.P - 6. The coupling was qualitatively checked by Kaiser Test as described in G.P - 10 after each coupling. After the final Fmoc-deprotection the resin was washed with a mixture of DCM/DIPEA/MeOH $(5 \times 2.0 \mathrm{ml})$, DMF $(5 \times 2.0 \mathrm{~mL})$ and DCM $(5 \times 2.0 \mathrm{~mL})$. Furthermore, the resin was dried overnight under reduced pressure. Next day, The cleavage was carried out via G.P - 12 to obtain the crude peptide as a white solid that was purified by semi-preparative HPLC to yield desired pure peptide as a colourless solid.

HPLC: (Column: Semi-preparative MN Nucleodur ${ }^{\circledR}$ 100-5-C18, 250mm $\times 10 \mathrm{~mm}, 5 \mu \mathrm{m}$, flow rate: $3 \mathrm{~mL} / \mathrm{min}$; Gradient: $80-97 \%$ B in $65 \mathrm{~min} ; \boldsymbol{\lambda}: 215,254,280 \mathrm{~nm}$ ); $\mathbf{t}_{\mathbf{R}}=36.6 \mathrm{~min}$

ESI-MS (m/z): $824.5[\mathrm{M}+4 \mathrm{H}]^{4+}, 1099.2[\mathrm{M}+3 \mathrm{H}]^{3+}, 1648.3[\mathrm{M}+2 \mathrm{H}]^{2+}$

HR-MS (m/z): Calculated for [M+2H] ${ }^{2+}: 1648.3875$, found: 1648.3867; Calculated for $[\mathrm{M}+3 \mathrm{H}]^{3+}$ : 1099.2733, found: 1099.2740; Calculated for $[\mathrm{M}+4 \mathrm{H}]^{4+}: 824.5270$ found: 824.5262 


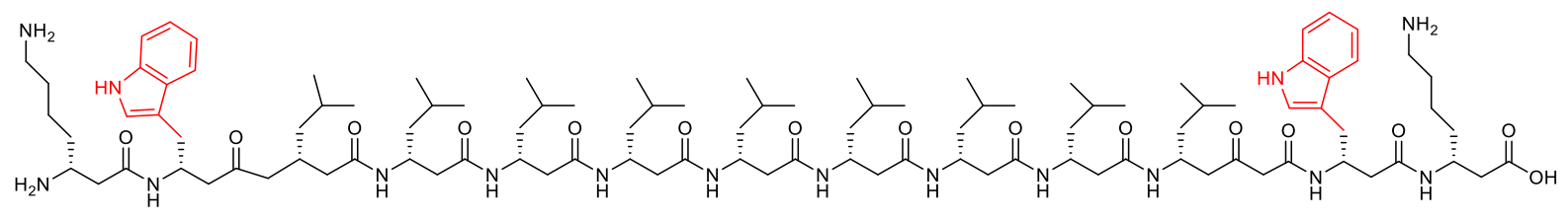

$$
\mathrm{C}_{104} \mathrm{H}_{174} \mathrm{~N}_{16} \mathrm{O}_{15}[1887.33 \mathrm{~g} / \mathrm{mmol}]
$$

Synthesis: The rink amide Nova PEG LL resin (1 eq., $1.1 \mathrm{~g}, 0.2 \mathrm{mmol}$ ) was loaded with Fmoc-D-

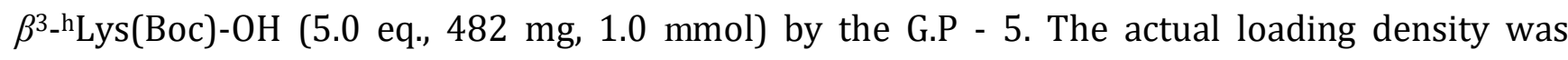
estimated to be $0.13 \mathrm{mmol} / \mathrm{g}$ following the method described in 6.4.2. The peptide was synthesized by performing double coupling with Fmoc-D- $\beta^{3}$-h Lys(Boc)-OH (5.0 eq. $(\times 2), 337 \mathrm{mg}$ $(\times 2), 0.7 \mathrm{mmol}(\times 2))$, Fmoc-D- $\beta^{3}$ h $\mathrm{Trp}($ Boc)$)-\mathrm{OH}(5$ eq. $(\times 2), 378 \mathrm{mg}(\times 2), 0.7 \mathrm{mmol}(\times 2))$, FmocD- $\beta^{3}$-hLeu-OH (5 eq. $(\times 2), 257 \mathrm{mg}(\times 2), 0.7 \mathrm{mmol}(\times 2)$ ) using G.P - 8(2) via microwave-assisted SPPS. The capping of the unreacted free amines were carried out following G.P - 6. The coupling was qualitatively checked by Kaiser Test as described in G.P - 10 after each coupling. After the final Fmoc-deprotection the resin was washed with a mixture of DCM/DIPEA/MeOH (5 × $2.0 \mathrm{ml})$, DMF $(5 \times 2.0 \mathrm{~mL})$ and DCM $(5 \times 2.0 \mathrm{~mL})$. Furthermore, the resin was dried overnight under reduced pressure. Next day, The cleavage was carried out via G.P - 12 to obtain the crude peptide as a white solid that was purified by semi-preparative HPLC to yield desired pure peptide as a colourless solid.

HPLC: (Column: Semi-preparative MN Nucleodur ${ }^{\circledR}$ 100-5-C18, 250mm $\times 10 \mathrm{~mm}, 5 \mu \mathrm{m}$, flow rate: $3 \mathrm{~mL} / \mathrm{min}$; Gradient: $80-97 \%$ B in $65 \mathrm{~min} ; \boldsymbol{\lambda}: 215,254,280 \mathrm{~nm}$ ); $\mathbf{t}_{\mathbf{R}}=32.8 \mathrm{~min}$

ESI-MS (m/z): 630.4[M+3H $]^{3+}, 944.1[\mathrm{M}+2 \mathrm{H}]^{2+}, 1889.4[\mathrm{M}+\mathrm{H}]^{+}$

HR-MS (m/z): Calculated for [M+H]+: 1889.4055, found: 1889.4063; $[\mathrm{M}+2 \mathrm{H}]^{2+}:$ 944.1635, found: 944.1631.

8.4.2.1.6 HO- $\left(6^{3 \mathrm{~h}} \mathrm{~V}-6^{3 \mathrm{~h}} \mathrm{~A}-6^{3 \mathrm{~h}} \mathrm{~L}\right)_{2}-\mathrm{NH}_{2}(\mathrm{P} 8)$<smiles>CC(C)CC(N)CC(=O)N[C@H](C)CCC(=O)N[C@@H](CC(=O)N[C@@H](CC(=O)N[C@H](C)CC(=O)N[C@H](CC(=O)O)C(C)C)CC(C)C)C(C)C</smiles> 
Synthesis: The 2-chlorotrityl chloride resin (1 eq., $122 \mathrm{mg}, 0.2 \mathrm{mmol}$ ) was pre-loaded with Fmoc-D- $\beta^{3}$-hVal-OH (3.0 eq., $212 \mathrm{mg}, 0.6 \mathrm{mmol}$ ) by the G.P - 7. The actual loading density was estimated to be $0.94 \mathrm{mmol} / \mathrm{g}$ following the method described in 6.4.2. The peptide was synthesized following the G.P - 9 using Fmoc-D- $\beta^{3}$ hAla-OH (3.0 eq., 107 mg, 0.33 mmol), Fmoc-D$\beta^{3}$ hLeu-OH (3.0 eq., $121 \mathrm{mg}, 0.33 \mathrm{mmol}$ ) and Fmoc-D- $\beta^{3}$ hVal-OH (3.0 eq., $116 \mathrm{mg}, 0.33 \mathrm{mmol}$ ) at room temperature without microwave assistance. The capping of the unreacted free amines were carried out following G.P - 6. The coupling was qualitatively checked by Kaiser Test as described in G.P - 10 after each coupling. After the final Fmoc-deprotection the resin was washed with a mixture of DCM/DIPEA/MeOH $(5 \times 2.0 \mathrm{ml})$, DMF $(5 \times 2.0 \mathrm{~mL})$ and DCM $(5 \times 2.0 \mathrm{~mL})$. Furthermore, the resin was dried overnight under reduced pressure. Next day, The cleavage was carried out via G.P - 12 to obtain the crude peptide as a white solid that was purified by semipreparative HPLC to yield desired pure peptide as a colourless solid.

HPLC: (Column: Semi-preparative MN Nucleodur ${ }^{\circledR}$ 100-5-C18, 250mm $\times 10 \mathrm{~mm}, 5 \mu \mathrm{m}$, flow rate: $3 \mathrm{~mL} / \mathrm{min}$; Gradient: $30-90 \%$ B in $55 \mathrm{~min} ; \boldsymbol{\lambda}: 215,254,280 \mathrm{~nm}$ ); $\mathbf{t}_{\mathbf{R}}=35.2 \mathrm{~min}$

ESI-MS (m/z): 334.5[M+2H $]^{2+}, 669.7[\mathrm{M}+\mathrm{H}]^{+}$

HR-MS (m/z): Calculated for $[\mathrm{M}+\mathrm{H}]^{+}:$669.7079, found: 669.7071; calculated for $[\mathrm{M}+2 \mathrm{H}]^{2+}: 334.5356$, found: 334.5359 .

8.4.2.1.7 HO- $\left(6^{3 \mathrm{~h}} \mathrm{~V}-6^{3 \mathrm{~h}} \mathrm{~A}-6^{3 \mathrm{~h}} \mathrm{~L}\right){ }_{2}$-FAM (P9)

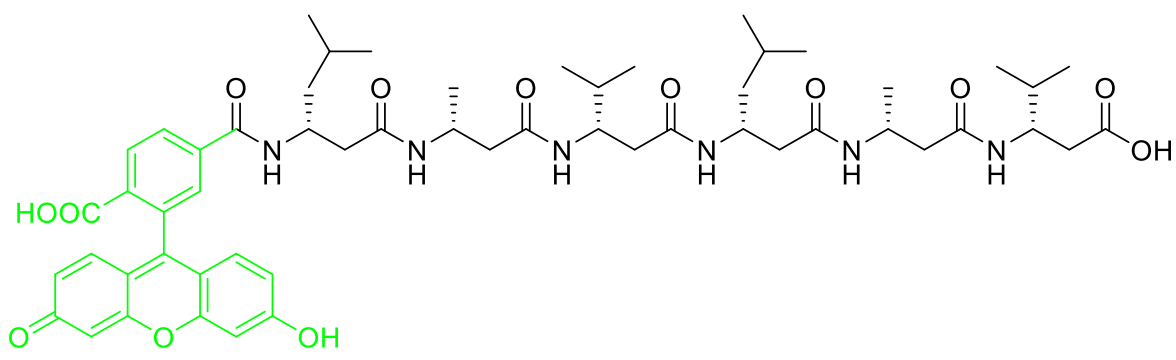

$\mathrm{C}_{55} \mathrm{H}_{74} \mathrm{~N}_{6} \mathrm{O}_{13}$ [1026.23] 
Synthesis The 2-chlorotrityl chloride resin (1 eq., $122 \mathrm{mg}, 0.2 \mathrm{mmol}$ ) was pre-loaded with Fmoc-D- $\beta^{3}$-hVal-OH (3.0 eq., $212 \mathrm{mg}, 0.6 \mathrm{mmol}$ ) by the G.P - 7. The actual loading density was estimated to be $0.94 \mathrm{mmol} / \mathrm{g}$ following the method described in 6.4.2. The peptide was synthesized following the G.P - 9 using Fmoc-D- $\beta^{3}$ hAla-OH (3.0 eq., 107 mg, 0.33 mmol), Fmoc-D$\beta^{3}$ hLeu-OH (3.0 eq., $121 \mathrm{mg}, 0.33 \mathrm{mmol}$ ) and Fmoc-D- $\beta^{3}$ hVal-OH (3.0 eq., $116 \mathrm{mg}, 0.33 \mathrm{mmol}$ ) at room temperature without microwave assistance. The capping of the unreacted free amines were carried out following G.P - 6. The coupling was qualitatively checked by Kaiser Test as described in G.P - 10 after each coupling. After the final Fmoc-deprotection the resin was washed with a mixture of DMF $(5 \times 2.0 \mathrm{~mL})$ and DCM $(5 \times 2.0 \mathrm{~mL})$. The fluorophore, 5(6)-carboxy fluorescein, was attached to the N-terminal of the peptide following the G.P - 11 with 5(6)carboxy fluoroscein (5.0 eq., $206 \mathrm{mg}, 0.55 \mathrm{mmol}$ ). After the completion of attachment reaction, the resin was washed with a mixture of DCM/DIPEA/MeOH $(10 \times 2.0 \mathrm{ml})$, DMF $(10 \times 2.0 \mathrm{~mL})$ and DCM $(10 \times 2.0 \mathrm{~mL})$. The resin was further dried overnight under reduced pressure. Next day, The cleavage was carried out via G.P - 12 to obtain the crude peptide as a orange solid that was purified by semi-preparative HPLC to yield desired pure peptide as a bright orange-yellow solid.

HPLC: (Column: Semi-preparative MN Nucleodur ${ }^{\circledR}$ 100-5-C18, 250mm $\times 10 \mathrm{~mm}, 5 \mu \mathrm{m}$, flow rate: $3 \mathrm{~mL} / \mathrm{min}$; Gradient: $15-97 \%$ B in $65 \mathrm{~min} ; \boldsymbol{\lambda}: 215,254,280 \mathrm{~nm}$ ); $\mathbf{t}_{\mathbf{R}}=36.3 \mathrm{~min}$

ESI-MS (m/z): $514.2[\mathrm{M}+2 \mathrm{H}]^{2+}, 1027.8[\mathrm{M}+\mathrm{H}]^{+}$

HR-MS (m/z): Calculated for $[\mathrm{M}+\mathrm{H}]^{+}: 1027.2966$, found: 1027.2975; calculated for $[\mathrm{M}+2 \mathrm{H}]^{2+}: 514.2017$, found: 514.2011

\subsubsection{Synthesis of $B^{2} / B^{3}$ and $B^{3} / B^{2}$-peptides}

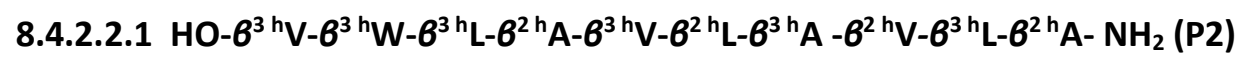

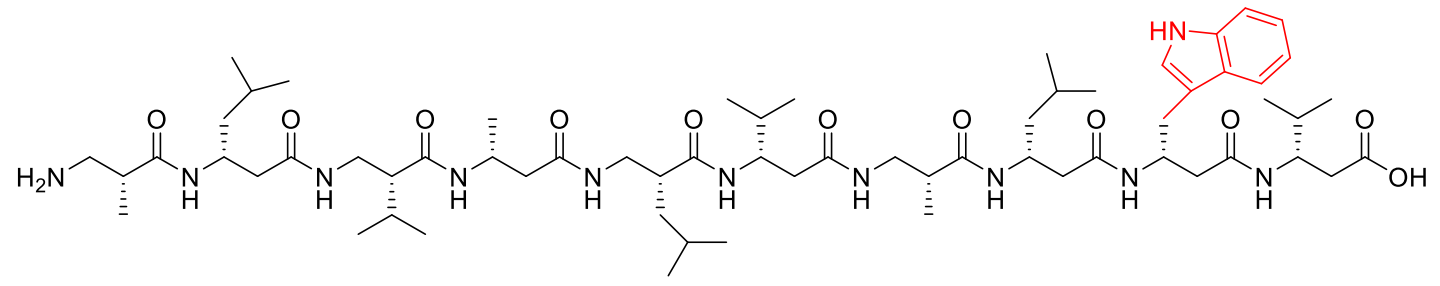


Synthesis: The 2-chlorotrityl chloride resin (1 eq., $122 \mathrm{mg}, 0.2 \mathrm{mmol}$ ) was pre-loaded with Fmoc-D- $\beta^{3}$-hVal-OH (3.0 eq., $212 \mathrm{mg}, 0.6 \mathrm{mmol}$ ) by the G.P - 7. The actual loading density was estimated to be $0.96 \mathrm{mmol} / \mathrm{g}$ following the method described in 6.4.2. The peptide was synthesized by performing a double coupling with Fmoc-D- $\beta^{3}$-hTrp(Boc)-OH (5.0 eq., 3.0 eq., 297 mg, 178 mg, 0.55 mmol, 0.33 mmol) and Fmoc-D- $\beta^{3}$ hLeu-OH (5.0 eq., 3.0 eq., 202 mg, 121 mg, $0.55 \mathrm{mmol}, 0.33 \mathrm{mmol}$ ) using G.P - 8(1) via microwave-assisted SPPS. The rest of the amino acids, like, Fmoc-D- $\beta^{3}$ hAla-OH (3.0 eq., 107 mg, 0.33 mmol), Fmoc-D- $\beta^{3}$ hLeu-OH (3.0 eq., 121 mg, $0.33 \mathrm{mmol}$ ) and Fmoc-D- $\beta^{3}$ hVal-OH (3.0 eq., $116 \mathrm{mg}, 0.33 \mathrm{mmol}$ ), were coupled using G.P - 9 via non-microwave assisted manual SPPS. The capping of the unreacted free amines were carried out following G.P - 6. The coupling was qualitatively checked by Kaiser Test as described in G.P 10 after each coupling. After the final Fmoc-deprotection the resin was washed with a mixture of DCM/DIPEA/MeOH $(5 \times 2.0 \mathrm{ml})$, DMF $(5 \times 2.0 \mathrm{~mL})$ and DCM $(5 \times 2.0 \mathrm{~mL})$. Furthermore, the resin was dried overnight under reduced pressure. Next day, The cleavage was carried out via G.P - 12 to obtain the crude peptide as a white solid that was purified by semi-preparative HPLC to yield desired pure peptide as a yellowish-white solid.

HPLC: (Column: Semi-preparative MN Nucleodur ${ }^{\circledR}$ 100-5-C18, 250mm $\times 10 \mathrm{~mm}, 5 \mu \mathrm{m}$, flow rate: $3 \mathrm{~mL} / \mathrm{min}$; Gradient: $15-97 \%$ B in $48 \mathrm{~min} ; \boldsymbol{\lambda}: 215,254,280 \mathrm{~nm}$ ); $\mathbf{t}_{\mathbf{R}}=26.5 \mathrm{~min}$

ESI-MS (m/z): $399.3[\mathrm{M}+3 \mathrm{H}]^{3+}, 597.5[\mathrm{M}+2 \mathrm{H}]^{2+}, 1195.7[\mathrm{M}+\mathrm{H}]^{+}$

HR-MS (m/z): Calculated for $[\mathrm{M}+\mathrm{H}]^{+}:$1195.7048, found: 1195.7041 , calculated for $[\mathrm{M}+2 \mathrm{H}]^{2+}$ : 597.5263, found: 597.5256.

8.4.2.2.2 HO- ${ }^{2 h} V-6^{3} h W-6^{3 h} L-6^{2 h} A-6^{3 h} V-6^{2 h} L-6^{3 h} A-6^{2 h} V-6^{3 h} L-6^{2 h} A-N_{2}$ (P3)

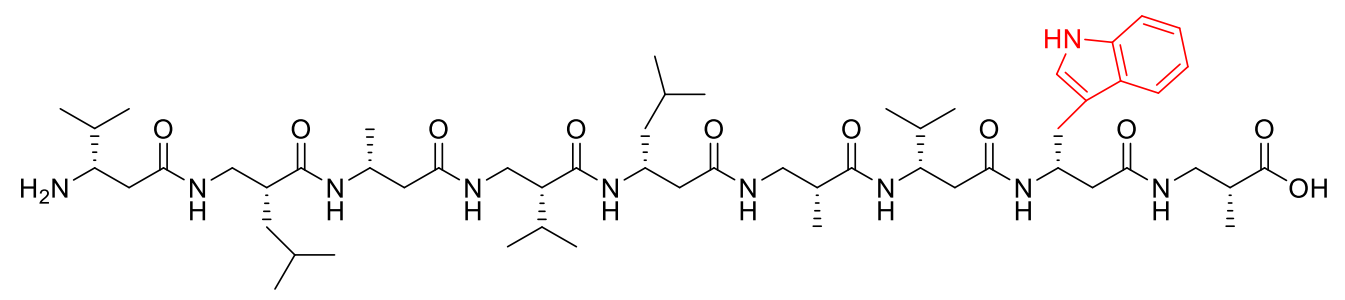

$\mathrm{C}_{56} \mathrm{H}_{94} \mathrm{~N}_{10} \mathrm{O}_{10}$ [1067.43] 
Synthesis: The 2-chlorotrityl chloride resin (1 eq., $061 \mathrm{mg}, 0.1 \mathrm{mmol}$ ) was pre-loaded with Fmoc-D- $\beta^{2}$ hAla-OH (3.0 eq., $97.6 \mathrm{mg}, 0.3 \mathrm{mmol}$ ) by the G.P - 7. The actual loading density was estimated to be $1.10 \mathrm{mmol} / \mathrm{g}$ following the method described in 6.4.2. The peptide was synthesized by performing a double coupling with Fmoc-D- $\beta^{3}$-hTrp(Boc)-OH (5.0 eq., 3.0 eq., 178 mg, $97.2 \mathrm{mg}, 0.33 \mathrm{mmol}, 0.18 \mathrm{mmol}$ ) and Fmoc-D- $\beta^{3}$ hVal-OH (5.0 eq., 3.0 eq., $116 \mathrm{mg}, 63.6 \mathrm{mg}$, $0.33 \mathrm{mmol}, 0.18 \mathrm{mmol}$ ) using G.P - 8(1) via microwave-assisted SPPS. The rest of the amino acids, Fmoc-D- $\beta^{3}$ hAla-OH (3.0 eq., 58.5 mg, 0.18 mmol), Fmoc-D- $\beta^{3}$ hLeu-OH (3.0 eq., 66.1 mg, $0.18 \mathrm{mmol}$ ) and Fmoc-D- $\beta^{3}$ hVal-OH (3.0 eq., $97.2 \mathrm{mg}, 0.18 \mathrm{mmol}$ ), were coupled using G.P - 9 via non-microwave assisted manual SPPS. The capping of the unreacted free amines were carried out following G.P - 6. The coupling was qualitatively checked by Kaiser Test as described in G.P 10 after each coupling. After the final Fmoc-deprotection the resin was washed with a mixture of DCM/DIPEA/MeOH $(5 \times 2.0 \mathrm{ml})$, DMF $(5 \times 2.0 \mathrm{~mL})$ and DCM $(5 \times 2.0 \mathrm{~mL})$. Furthermore, the resin was dried overnight under reduced pressure. Next day, The cleavage was carried out via G.P - 12 to obtain the crude peptide as a yellowish solid that was purified by semi-preparative HPLC to yield desired pure peptide as a colourless solid.

HPLC: (Column: Semi-preparative MN Nucleodur ${ }^{\circledR}$ 100-5-C18, 250mm $\times 10 \mathrm{~mm}, 5 \mu \mathrm{m}$, flow rate: $3 \mathrm{~mL} / \mathrm{min}$; Gradient: $23-93 \%$ B in $55 \mathrm{~min}$; $\lambda: 215,254,280 \mathrm{~nm}$ ); $\mathbf{t}_{\mathbf{R}}=33.8 \mathrm{~min}$

ESI-MS (m/z): $356.9[\mathrm{M}+3 \mathrm{H}]^{3+}, 533.6[\mathrm{M}+2 \mathrm{H}]^{2+}, 1068.5[\mathrm{M}+\mathrm{H}]^{+}$

HR-MS (m/z): Calculated for $[\mathrm{M}+\mathrm{H}]^{+}:$1068.5033, found: 1068.5041 , calculated for $[\mathrm{M}+2 \mathrm{H}]^{2+}$ : 533.6381, found: 533.6377.

8.4.2.2.3 HO- $6^{2 h} L-6^{3 h} A-6^{2 h} V-6^{3 h} L-6^{2 h} A-6^{3 h} V-N_{2}(P 6)$

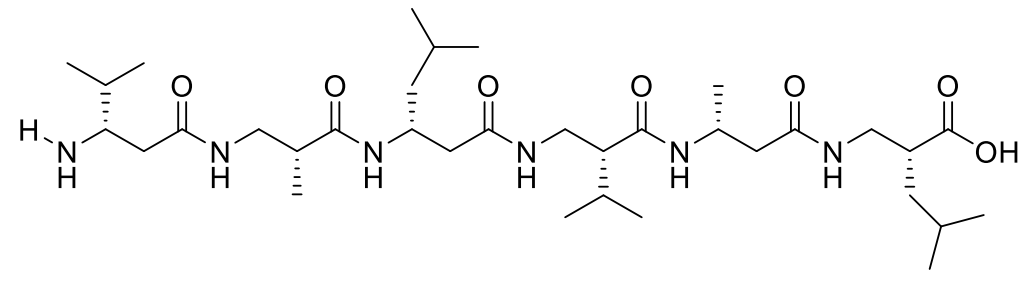

$\mathrm{C}_{34} \mathrm{H}_{64} \mathrm{~N}_{6} \mathrm{O}_{7}[668.92]$ 
Synthesis: The 2-chlorotrityl chloride resin (1 eq., $122 \mathrm{mg}, 0.2 \mathrm{mmol}$ ) was pre-loaded with Fmoc-D- $\beta^{3}$-hLeu-OH (3.0 eq., $220 \mathrm{mg}, 0.6 \mathrm{mmol}$ ) by the G.P - 7. The actual loading density was estimated to be $1.15 \mathrm{mmol} / \mathrm{g}$ following the method described in 6.4.2. The peptide was synthesized following the G.P - 9 using Fmoc-D- $\beta^{3}$ hAla-OH (3.0 eq., 136 mg, 0.42 mmol), Fmoc-D$\beta^{3}$ hLeu-OH (3.0 eq., $154 \mathrm{mg}, 0.42 \mathrm{mmol}$ ) and Fmoc-D- $\beta^{3}$ hVal-OH (3.0 eq., $148 \mathrm{mg}, 0.42 \mathrm{mmol}$ ) at room temperature without microwave assistance. The capping of the unreacted free amines were carried out following G.P - 6. The coupling was qualitatively checked by Kaiser Test as described in G.P - 10 after each coupling. After the final Fmoc-deprotection the resin was washed with a mixture of DCM/DIPEA/MeOH $(5 \times 2.0 \mathrm{ml})$, DMF $(5 \times 2.0 \mathrm{~mL})$ and DCM $(5 \times 2.0 \mathrm{~mL})$. Furthermore, the resin was dried overnight under reduced pressure. Next day, The cleavage was carried out via G.P - 12 to obtain the crude peptide as a white solid that was purified by semipreparative HPLC to yield desired pure peptide as a colourless solid.

HPLC: (Column: Semi-preparative MN Nucleodur ${ }^{\circledR}$ 100-5-C18, 250mm $\times 10 \mathrm{~mm}, 5 \mu \mathrm{m}$, flow rate: $3 \mathrm{~mL} / \mathrm{min}$; Gradient: $15-97 \%$ B in $48 \mathrm{~min} ; \boldsymbol{\lambda}: 215,254,280 \mathrm{~nm}$ ); $\mathbf{t}_{\mathbf{R}}=25.2 \mathrm{~min}$

ESI-MS (m/z): 334.7[M+2H $]^{2+}, 669.9[\mathrm{M}+\mathrm{H}]^{+}$

HR-MS (m/z): Calculated for $[\mathrm{M}+\mathrm{H}]^{+}$: 669.7588, found: 669.7594; calculated for $[\mathrm{M}+2 \mathrm{H}]^{2+}: 334.7183$, found: 334.7174 .

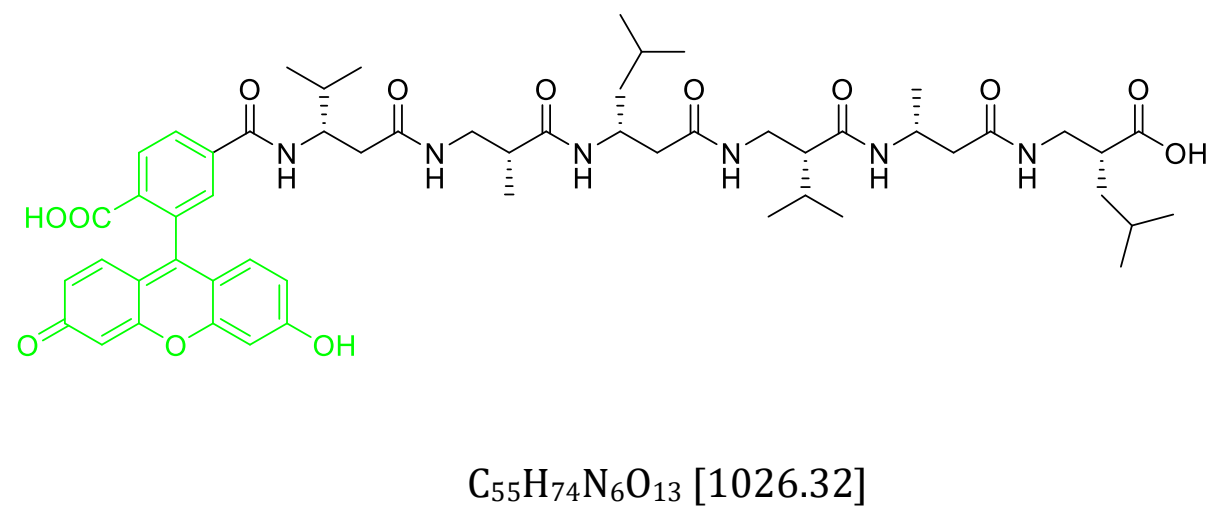

Synthesis: The 2-chlorotrityl chloride resin (1 eq., $122 \mathrm{mg}, 0.2 \mathrm{mmol}$ ) was pre-loaded with Fmoc-D- $\beta^{3}$-hLeu-OH (3.0 eq., $220 \mathrm{mg}, 0.6 \mathrm{mmol}$ ) by the G.P - 7. The actual loading density was 
estimated to be $1.15 \mathrm{mmol} / \mathrm{g}$ following the method described in 6.4.2. The peptide was synthesized following the G.P - 9 using Fmoc-D- $\beta^{3}$ hAla-OH (3.0 eq., 136 mg, 0.42 mmol), Fmoc-D$\beta^{3}$ hLeu-OH (3.0 eq., $154 \mathrm{mg}, 0.42 \mathrm{mmol}$ ) and Fmoc-D- $\beta^{3}$ hVal-OH (3.0 eq., $148 \mathrm{mg}, 0.42 \mathrm{mmol}$ ) at room temperature without microwave assistance. The capping of the unreacted free amines were carried out following G.P - 6. The coupling was qualitatively checked by Kaiser Test as described in G.P - 10 after each coupling. After the final Fmoc-deprotection the resin was washed with a mixture of DMF $(10 \times 2.0 \mathrm{~mL})$ and DCM $(10 \times 2.0 \mathrm{~mL})$. The fluorophore, 5(6)-carboxy fluorescein, was attached to the N-terminal of the peptide following the G.P - 11 with 5(6)carboxy fluoroscein (5.0 eq., $263 \mathrm{mg}, 0.7 \mathrm{mmol}$ ). After the completion of attachment reaction, the resin was washed with a mixture of DCM/DIPEA/MeOH $(5 \times 2.0 \mathrm{ml})$, DMF $(5 \times 2.0 \mathrm{~mL})$ and DCM $(5 \times 2.0 \mathrm{~mL})$. The resin was further dried overnight under reduced pressure. Next day, The cleavage was carried out via G.P - 12 to obtain the crude peptide as a orange solid that was purified by semi-preparative HPLC to yield desired pure peptide as a bright orange-yellow solid.

HPLC: (Column: Semi-preparative MN Nucleodur ${ }^{\circledR}$ 100-5-C18, 250mm $\times 10 \mathrm{~mm}$, $5 \mu \mathrm{m}$, flow rate: $3 \mathrm{~mL} / \mathrm{min}$; Gradient: $15-97 \%$ B in $56 \mathrm{~min} ; \boldsymbol{\lambda}: 215,254,280 \mathrm{~nm}$ ); $\mathbf{t}_{\mathbf{R}}=30.7 \mathrm{~min}$

ESI-MS (m/z): 514.7[M+2H $]^{2+}, 1027.4[\mathrm{M}+\mathrm{H}]^{+}$

HR-MS (m/z): Calculated for $[\mathrm{M}+\mathrm{H}]^{+}: 1027.4033$, found: 1027.4042; calculated for $[\mathrm{M}+2 \mathrm{H}]^{2+}: 514.7142$, found: 514.7148 .

\section{Appendix}

\subsection{Tryptophan fluorescence of $\boldsymbol{B}^{3}$ (Val/Ala/Leu triad) peptide P1 in TRIS buffer and three different lipid vesicles keeping $P / L$ ratio $1: 50$ and peptide concentration $12 \mu \mathrm{M}$ constant at $50^{\circ} \mathrm{C}$ temperature}



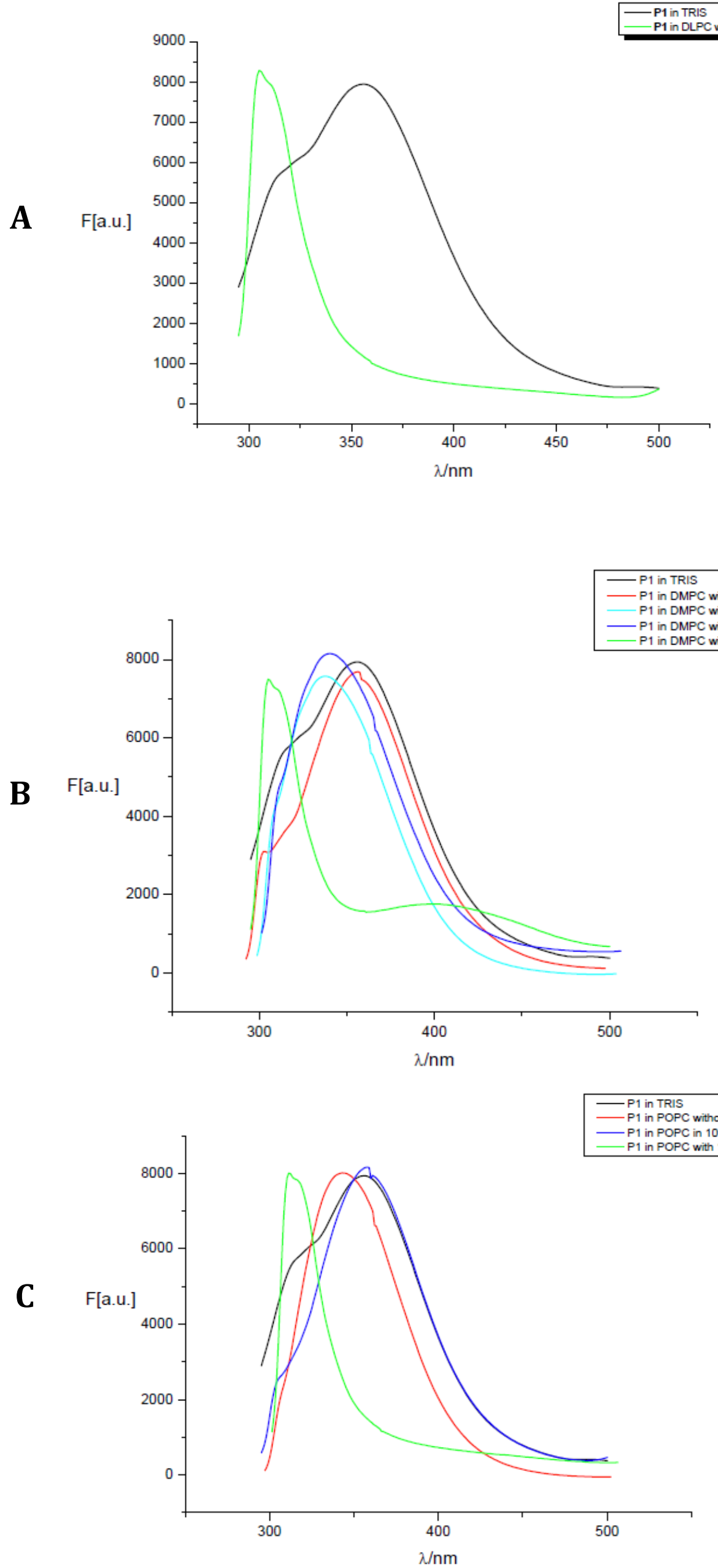
Fig. Comparison Tryptophan fluorescence of $\beta^{3}$ (Val/Ala/Leu triad) peptide P1 in TRIS buffer and in DLPC LUV without EtOH (A); in TRIS buffer and in DMPC LUV without and with 3\%, 5\% $10 \%$ EtOH, respectively (B); in TRIS buffer and in POPC LUV without and with $10 \%, 15 \%$ EtOH, respectively $(\mathbf{C})$

9.2 Tryptophan fluorescence of alternate $B^{3} / B^{2}$ (Val/Ala/Leu triad) peptide P2 in TRIS buffer and three different lipid vesicles keeping $P / L$ ratio $1: 50$ and peptide concentration $12 \mu \mathrm{M}$ constant at $50^{\circ} \mathrm{C}$ temperature

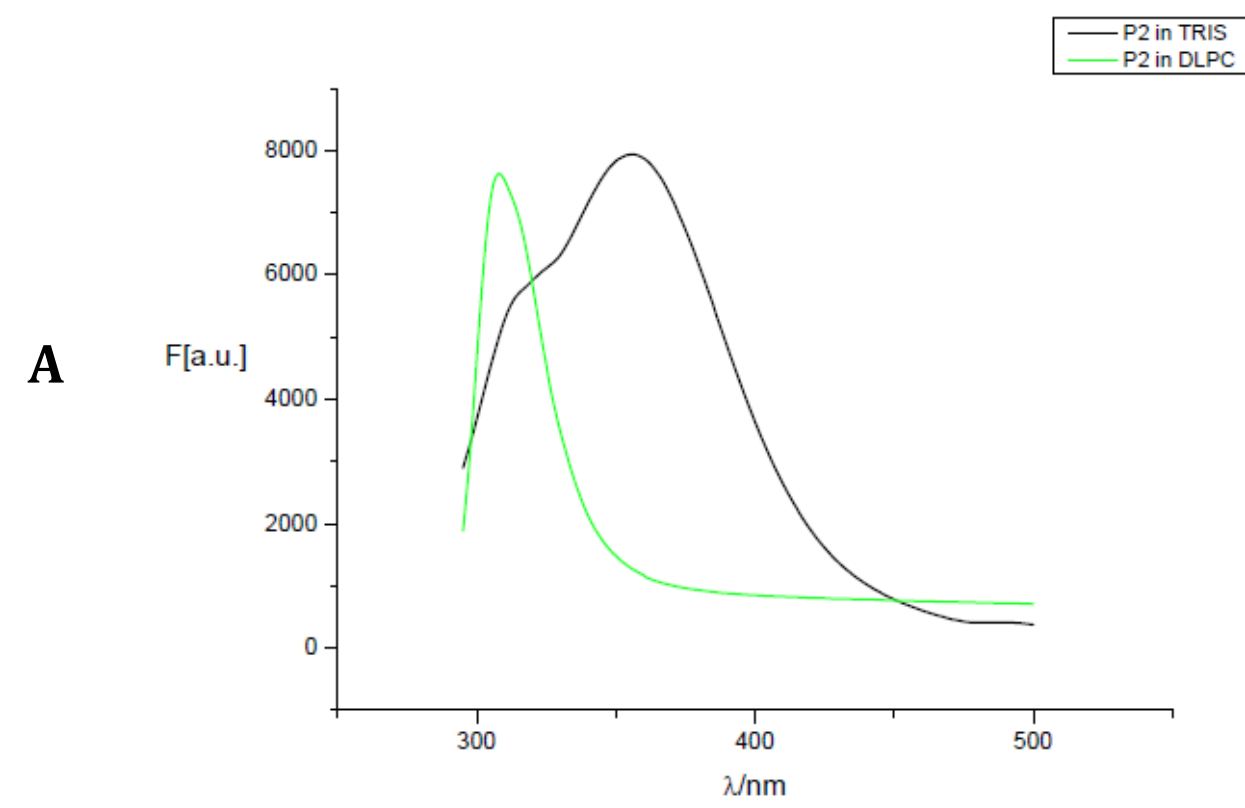



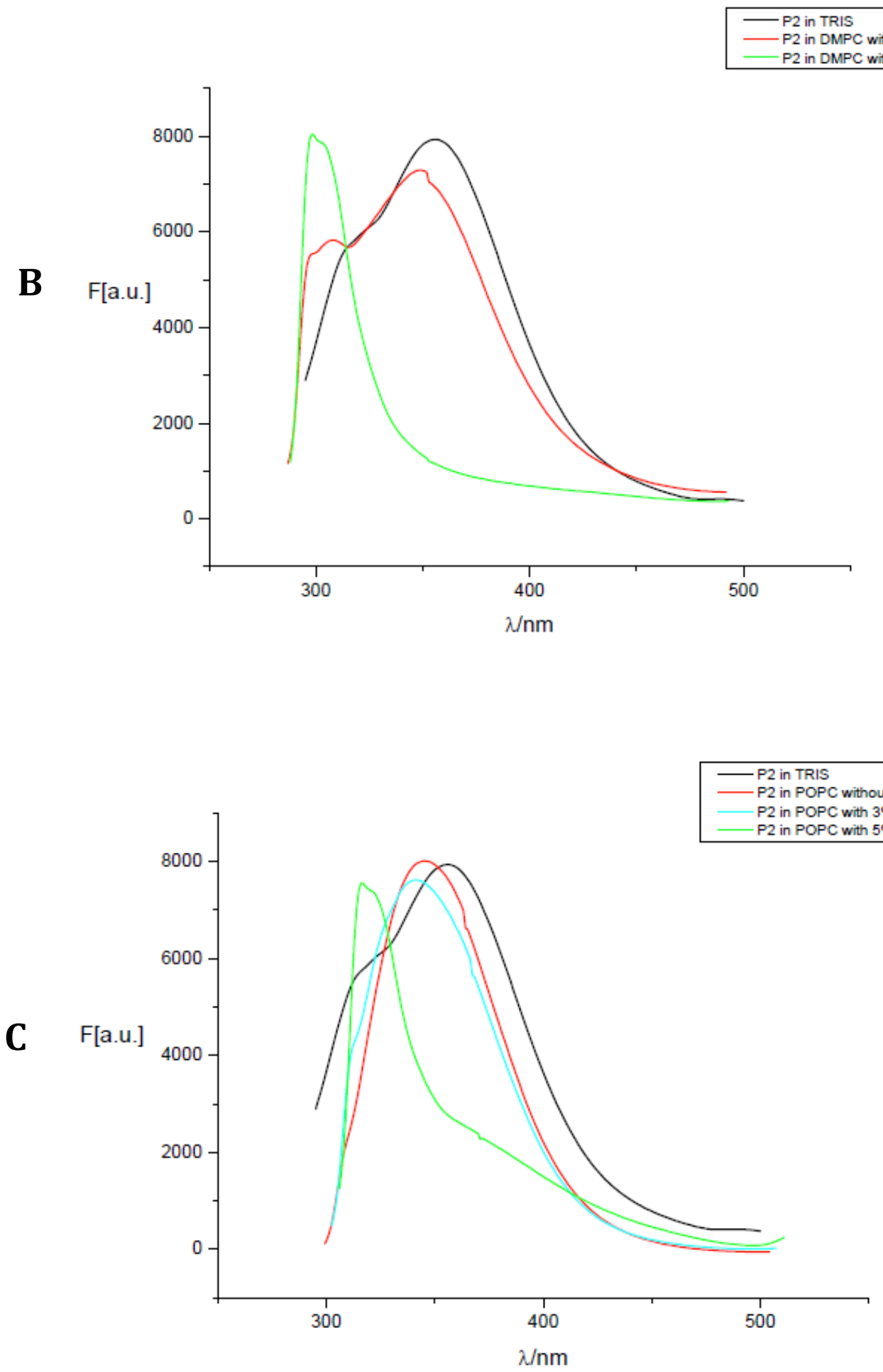

Figure 9.1: Comparison Tryptophan fluorescence of $\beta^{3} / \beta^{2}$ (Val/Ala/Leu triad) peptide P2 in TRIS buffer and in DLPC LUV without EtOH (A); in TRIS buffer and in DMPC LUV without and with 2\% EtOH, respectively (B); in TRIS buffer and in POPC LUV without and with 3\%, 5\% EtOH, respectively (C) 
9.3 Tryptophan fluorescence of alternate $B^{2} / b^{3}$ (Val/Ala/Leu triad) peptide P3 in TRIS buffer and three different lipid vesicles keeping $P / L$ ratio 1:50 and peptide concentration $12 \mu \mathrm{M}$ constant at $50^{\circ} \mathrm{C}$ temperature
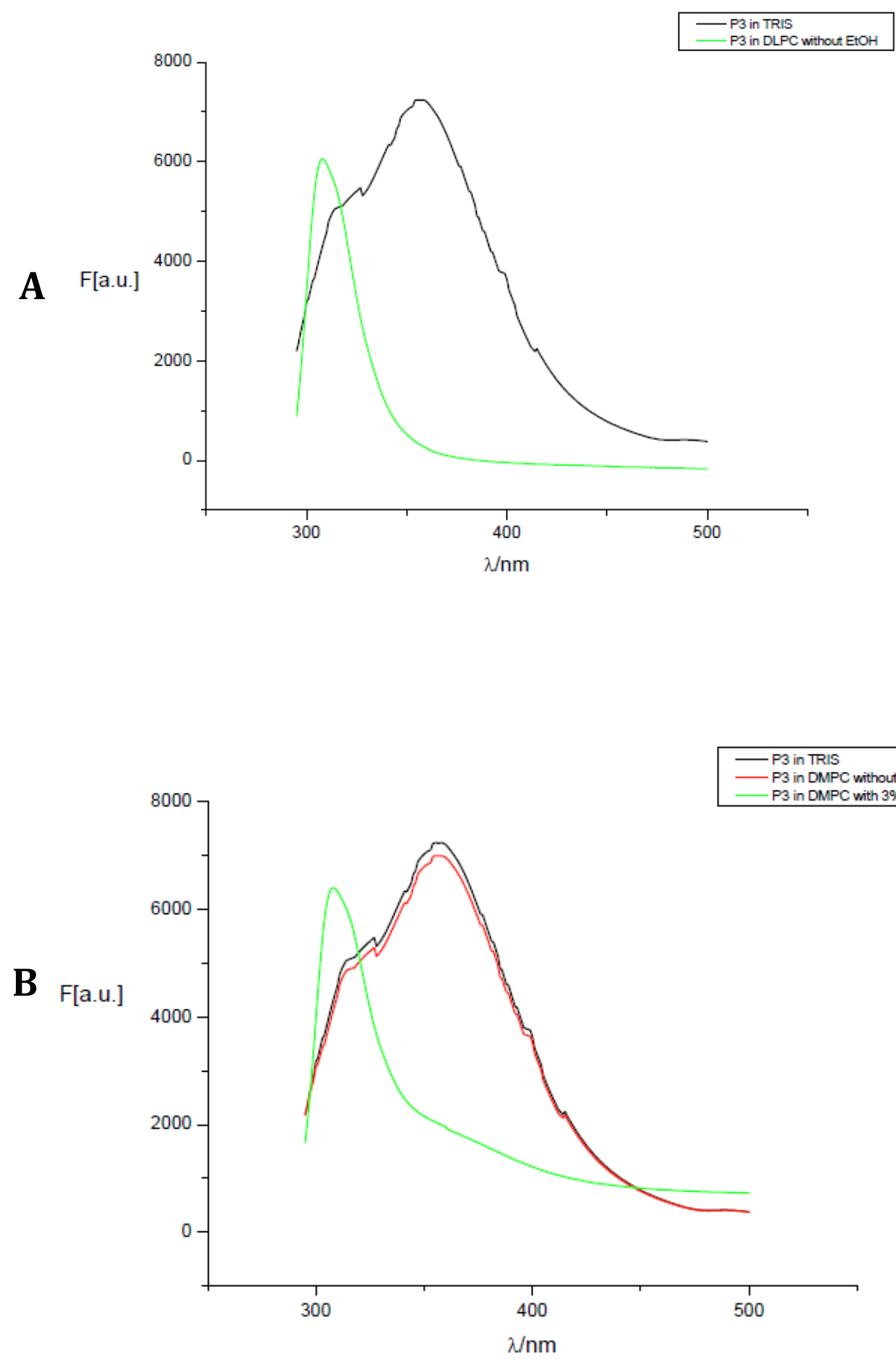


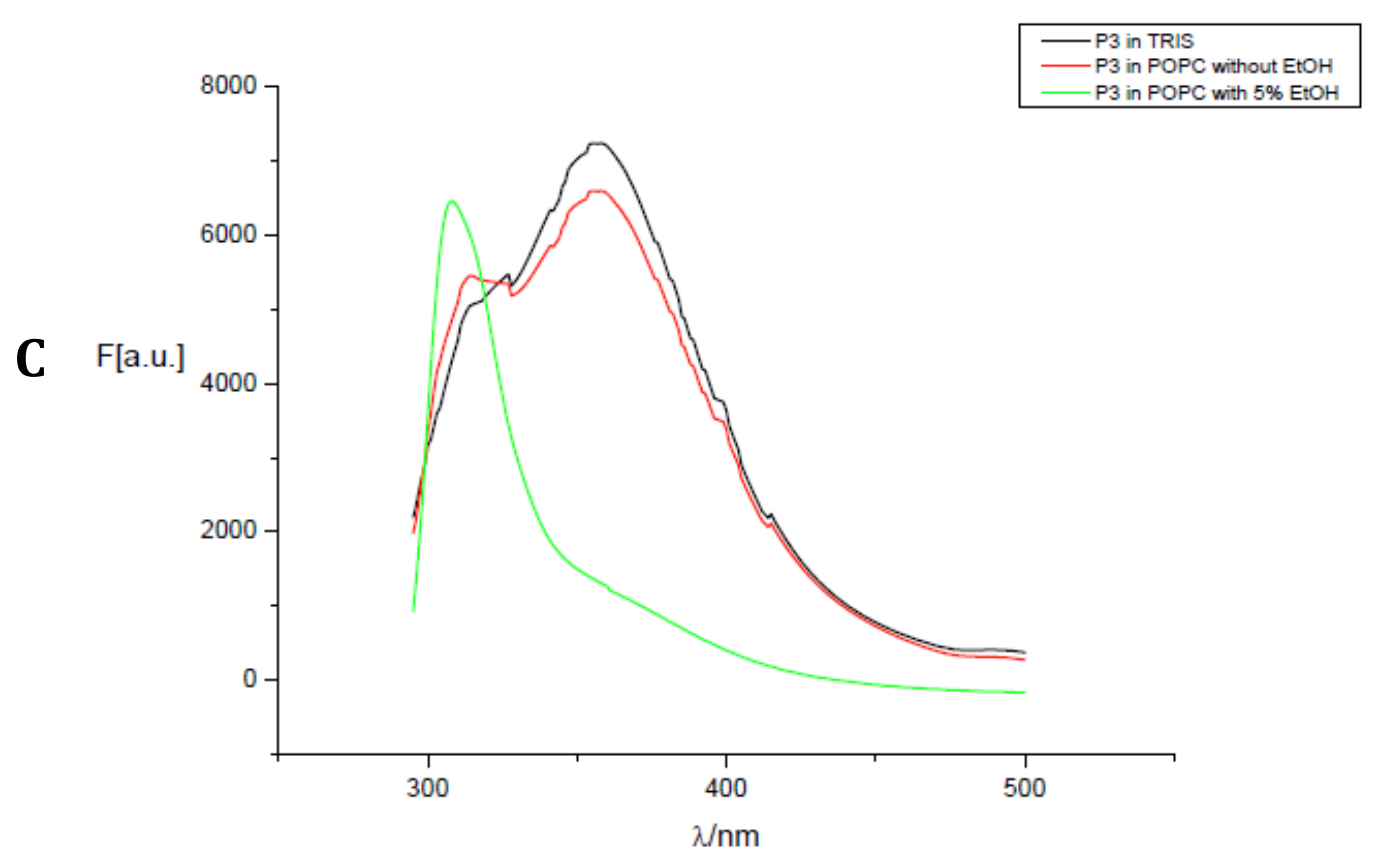

Figure 9.2: Comparison Tryptophan fluorescence of $\beta^{2} / \beta^{3}$ (Val/Ala/Leu triad) peptide P3 in TRIS buffer and in DLPC LUV without EtOH (A); in TRIS buffer and in DMPC LUV without and with 3\% EtOH, respectively (B); in TRIS buffer and in POPC LUV without and with 5\% EtOH, respectively (C)

9.4 Tryptophan fluorescence of $b^{3}$ (Val) peptide P10 in TRIS buffer and three different lipid vesicles keeping $\mathrm{P} / \mathrm{L}$ ratio $1: 50$ and peptide concentration $12 \mu \mathrm{M}$ constant at $50^{\circ} \mathrm{C}$ temperature

A

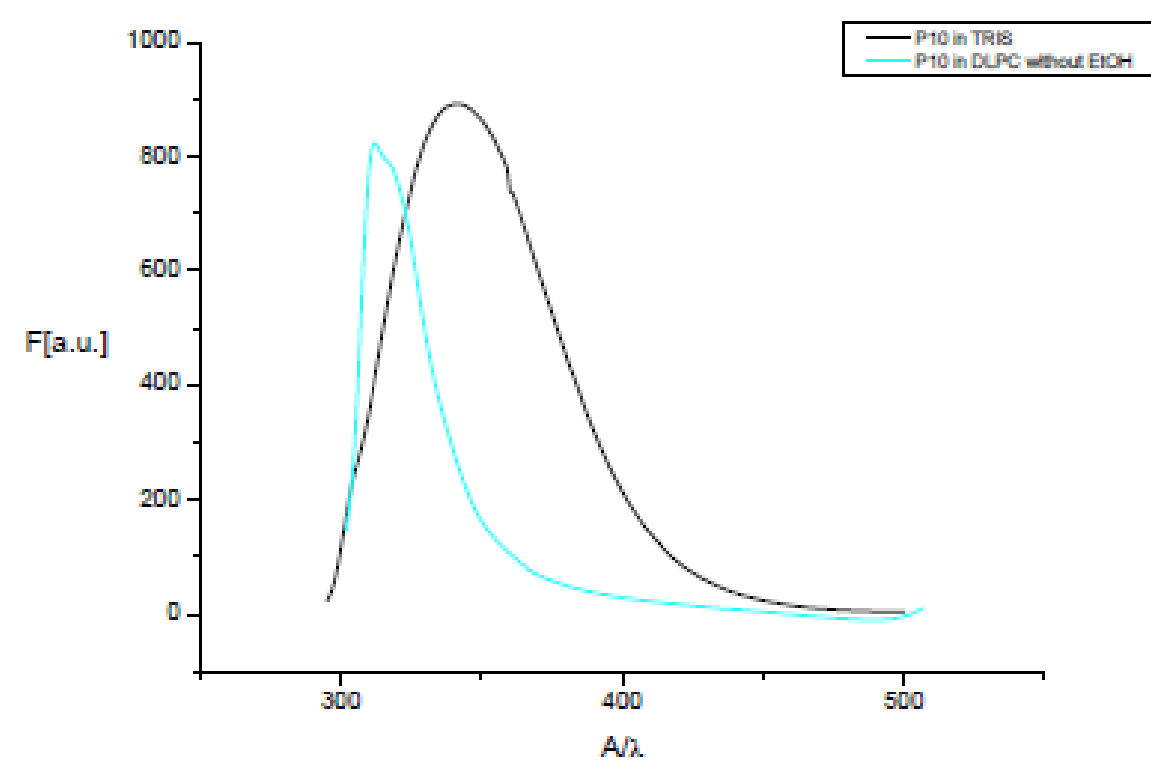



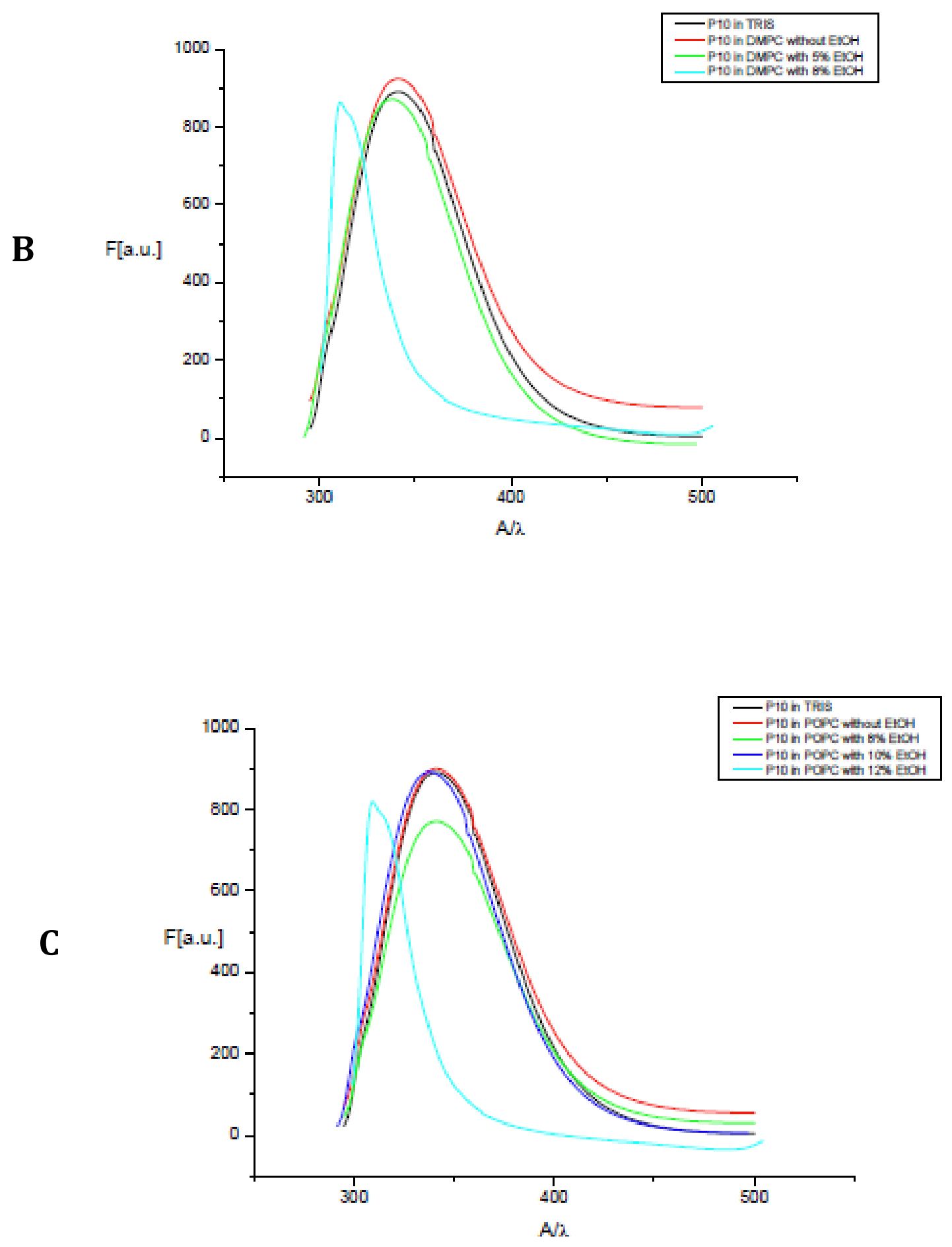

Figure 9.3: Comparison Tryptophan fluorescence of $\beta^{3}$ (Val) peptide P10 in TRIS buffer and in DLPC LUV without EtOH (A); in TRIS buffer and in DMPC LUV without and with 5\%, 8\% EtOH, respectively (B); in TRIS buffer and in POPC LUV without and with 8\%, 10\%, 12\% EtOH, respectively $(\boldsymbol{C})$ 
9.5 Tryptophan fluorescence of $\boldsymbol{b}^{3}$ (Val) peptide P4 in TRIS buffer and three different lipid vesicles keeping $P / L$ ratio $1: 50$ and peptide concentration $12 \mu \mathrm{M}$ constant at $50^{\circ} \mathrm{C}$ temperature

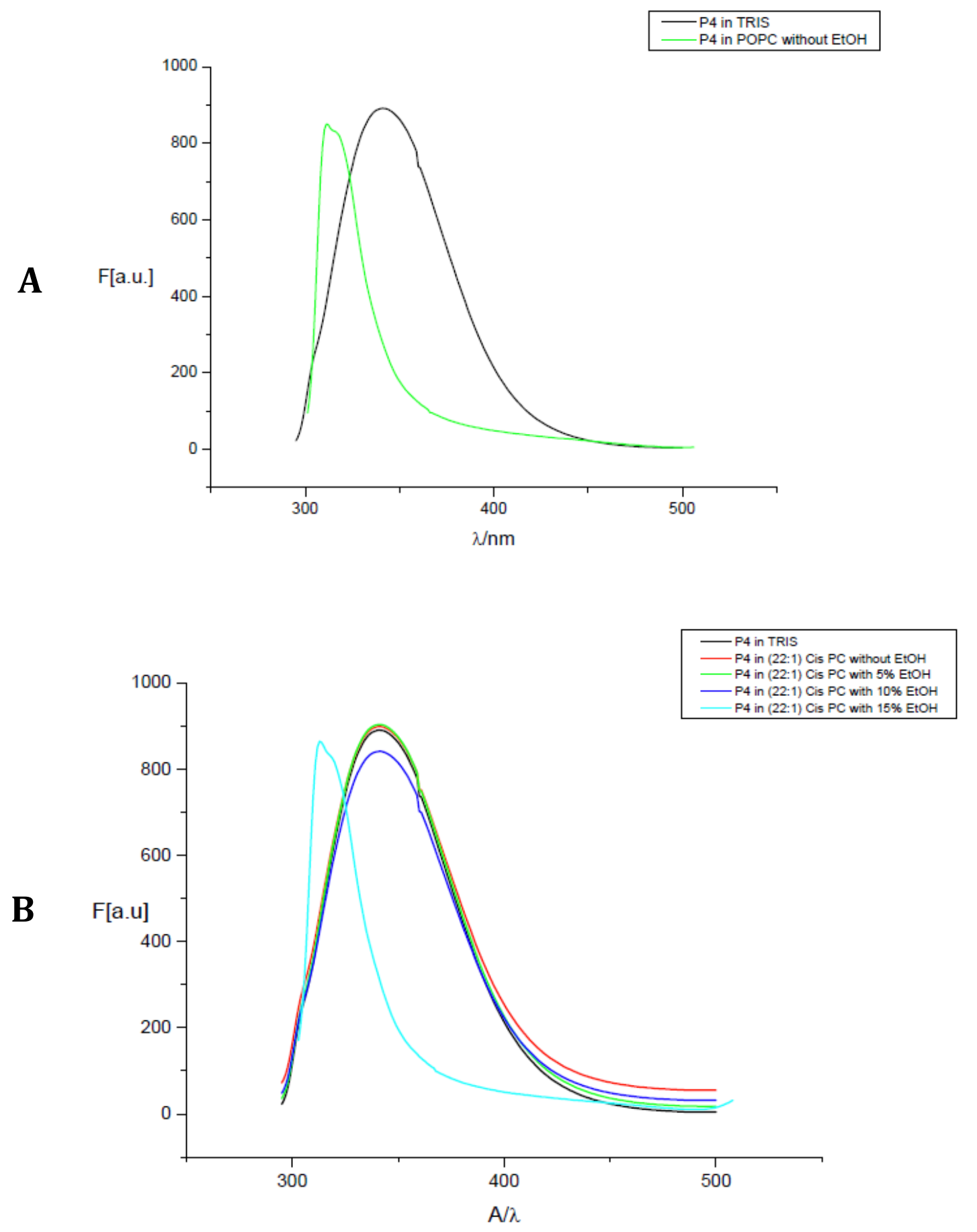




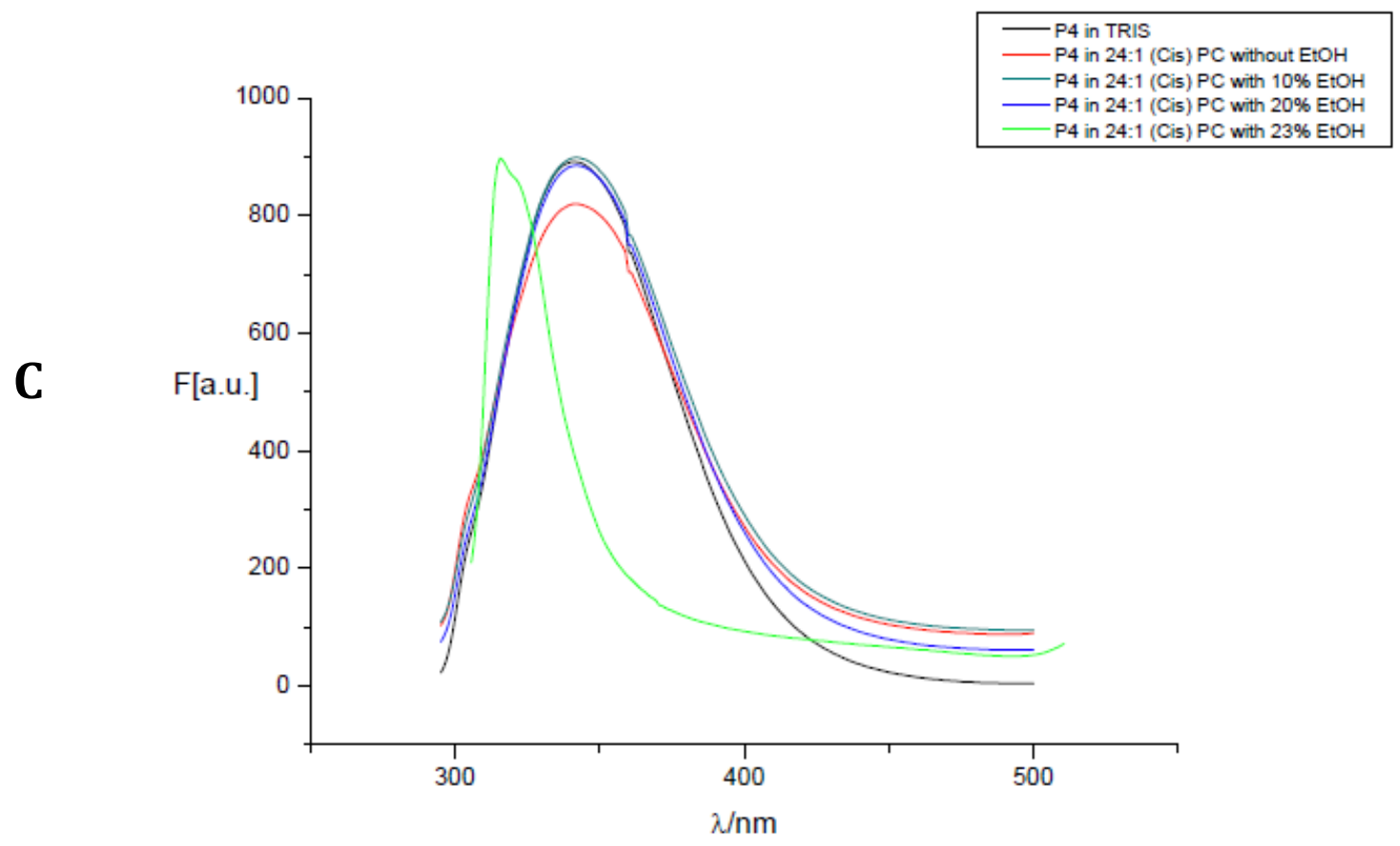

Figure 9.4: Comparison Tryptophan fluorescence of $\beta^{3}$ (Val) peptide P4 in TRIS buffer and in POPC LUV without EtOH (A); in TRIS buffer and in \{22:1 (Cis) PC\} LUV without and with 5\%, 10\% EtOH, respectively (B); in TRIS buffer and in POPC LUV without and with 10\%, 20\%, 23\% EtOH, respectively $(\boldsymbol{C})$

9.6 Tryptophan fluorescence of $B^{3}$ (Leu) peptide P11 in TRIS buffer and three different lipid vesicles keeping $\mathrm{P} / \mathrm{L}$ ratio $1: 50$ and peptide concentration $12 \mu \mathrm{M}$ constant at $50^{\circ} \mathrm{C}$ temperature 

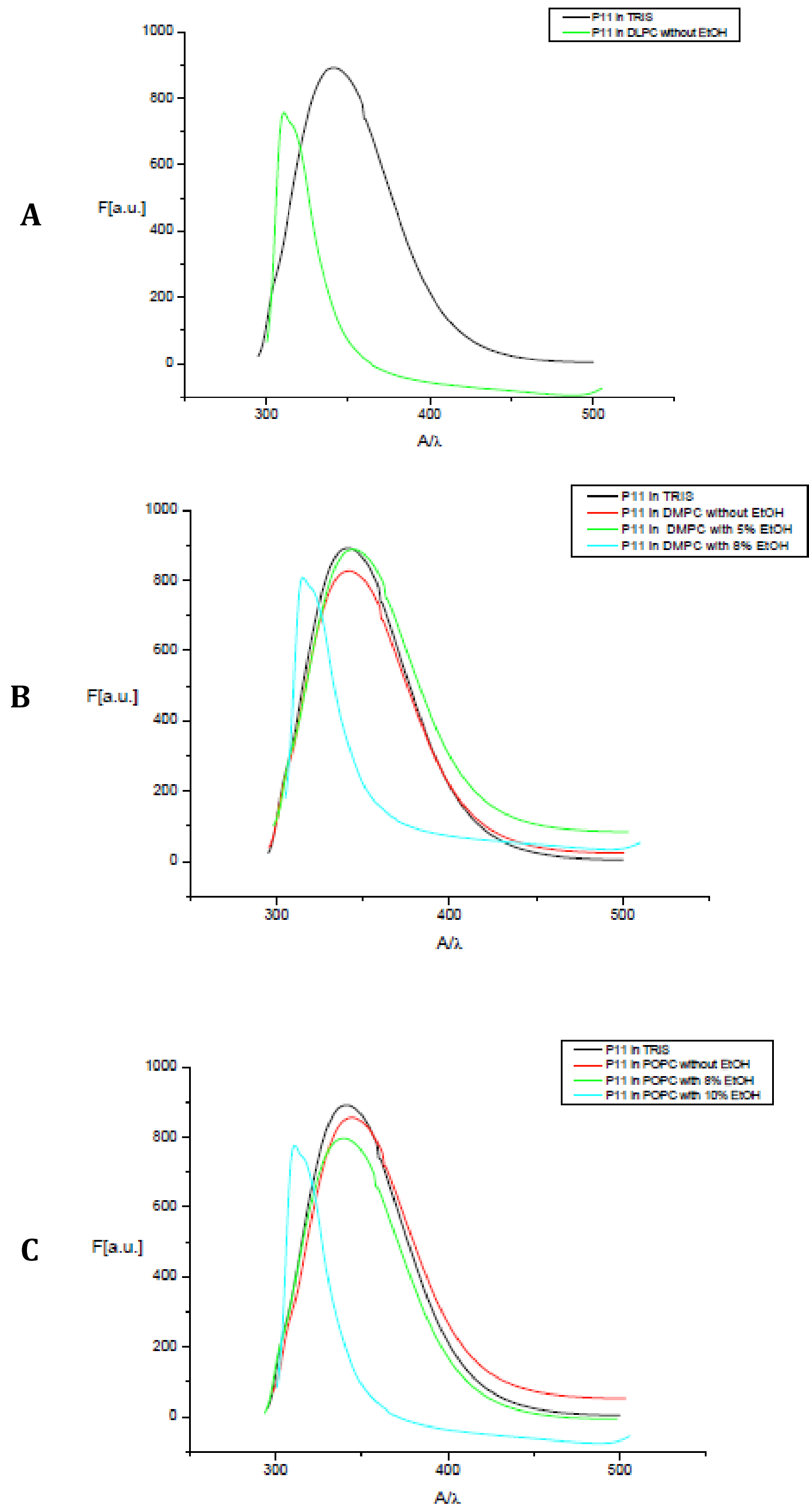
Figure 9.5 Comparison Tryptophan fluorescence of $\beta^{3}$ (Leu) peptide P11 in TRIS buffer and in DLPC LUV without EtOH (A); in TRIS buffer and in DMPC LUV without and with 5\%, 8\% EtOH, respectively (B); in TRIS buffer and in POPC LUV without and with 8\%, 10\%, EtOH, respectively (C)

9.7 Tryptophan fluorescence of $B^{3}$ (Leu) peptide P5 in TRIS buffer and three different lipid vesicles keeping $P / L$ ratio $1: 50$ and peptide concentration $12 \mu \mathrm{M}$ constant at $50^{\circ} \mathrm{C}$ temperature

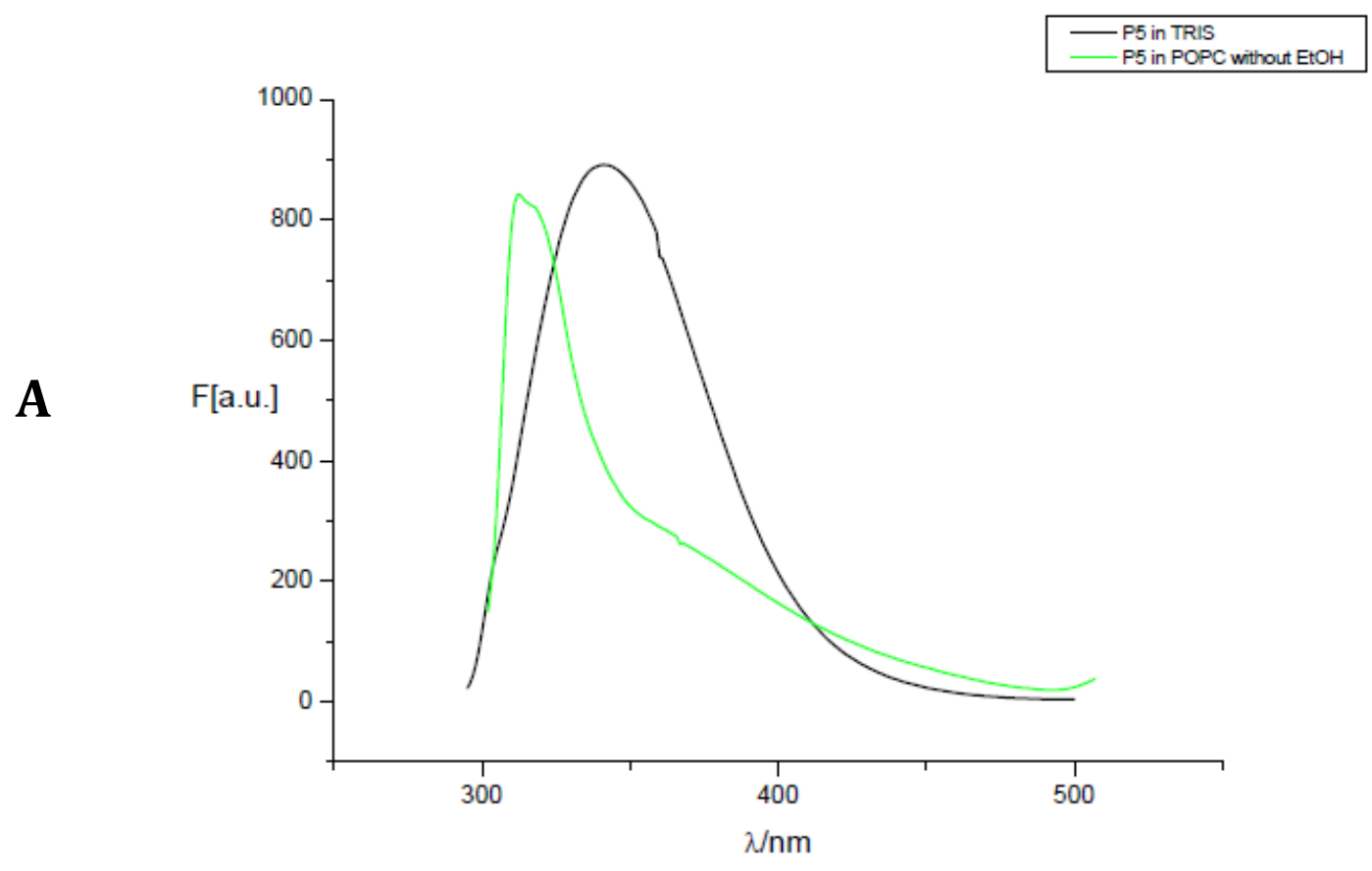



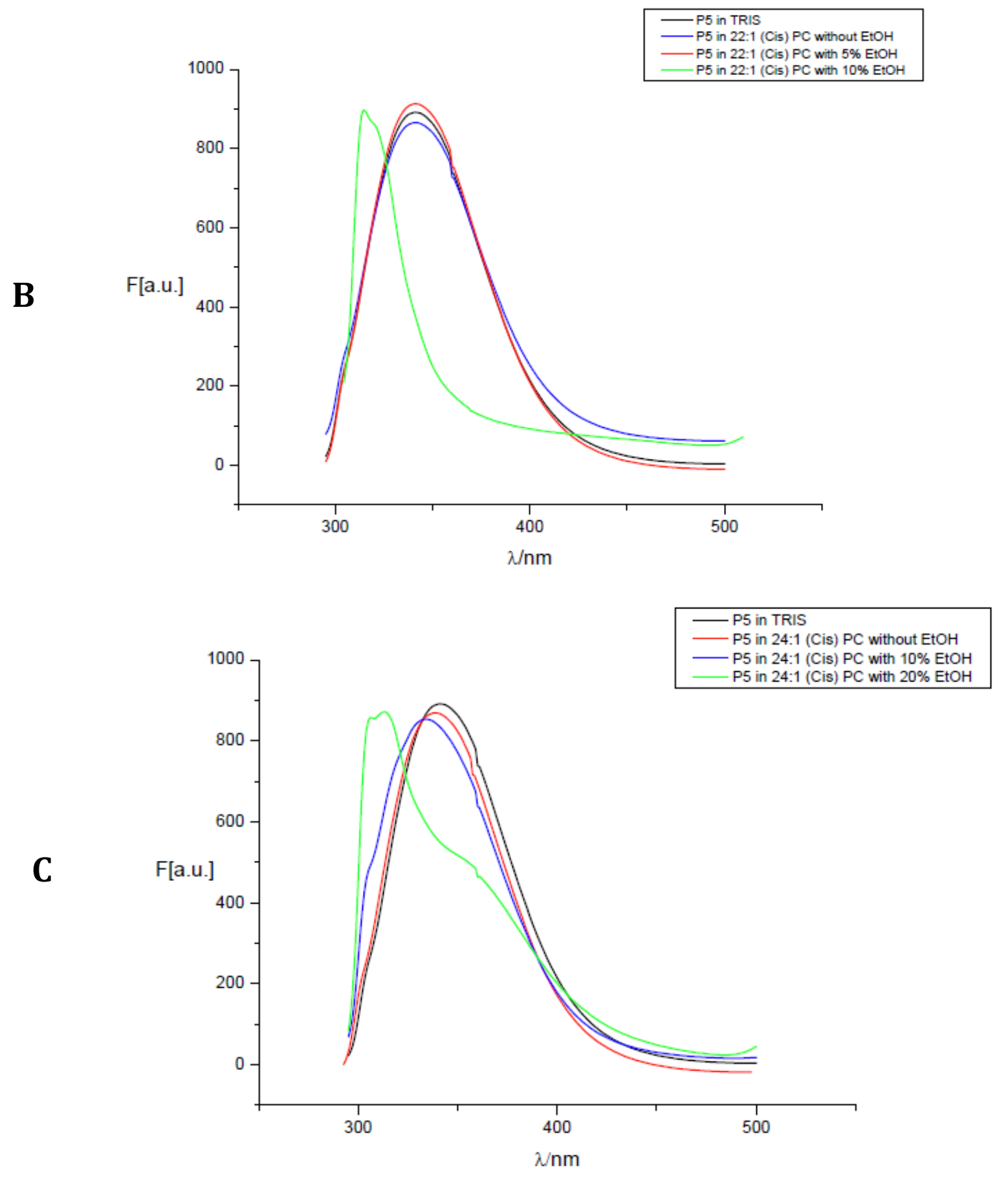

Figure 9.6: Comparison Tryptophan fluorescence of $\beta^{3}$ (Leu) peptide P5 in TRIS buffer and in POPC LUV without EtOH (A); in TRIS buffer and in $\{22: 1$ (Cis) PC\} LUV without and with 5\%, 10\% EtOH, respectively (B); in TRIS buffer and in POPC LUV without and with 10\%, 20\% EtOH, respectively (C) 
9.8 CD-spectra of $b^{3}$ (Val/Ala/Leu triad) peptide P1 in TFE and within three different lipid vesicles prepared in TRIS buffer keeping $P / L$ ratio 1:50 and peptide concentration $30 \mu \mathrm{M}$ constant at $50^{\circ} \mathrm{C}$ temperature
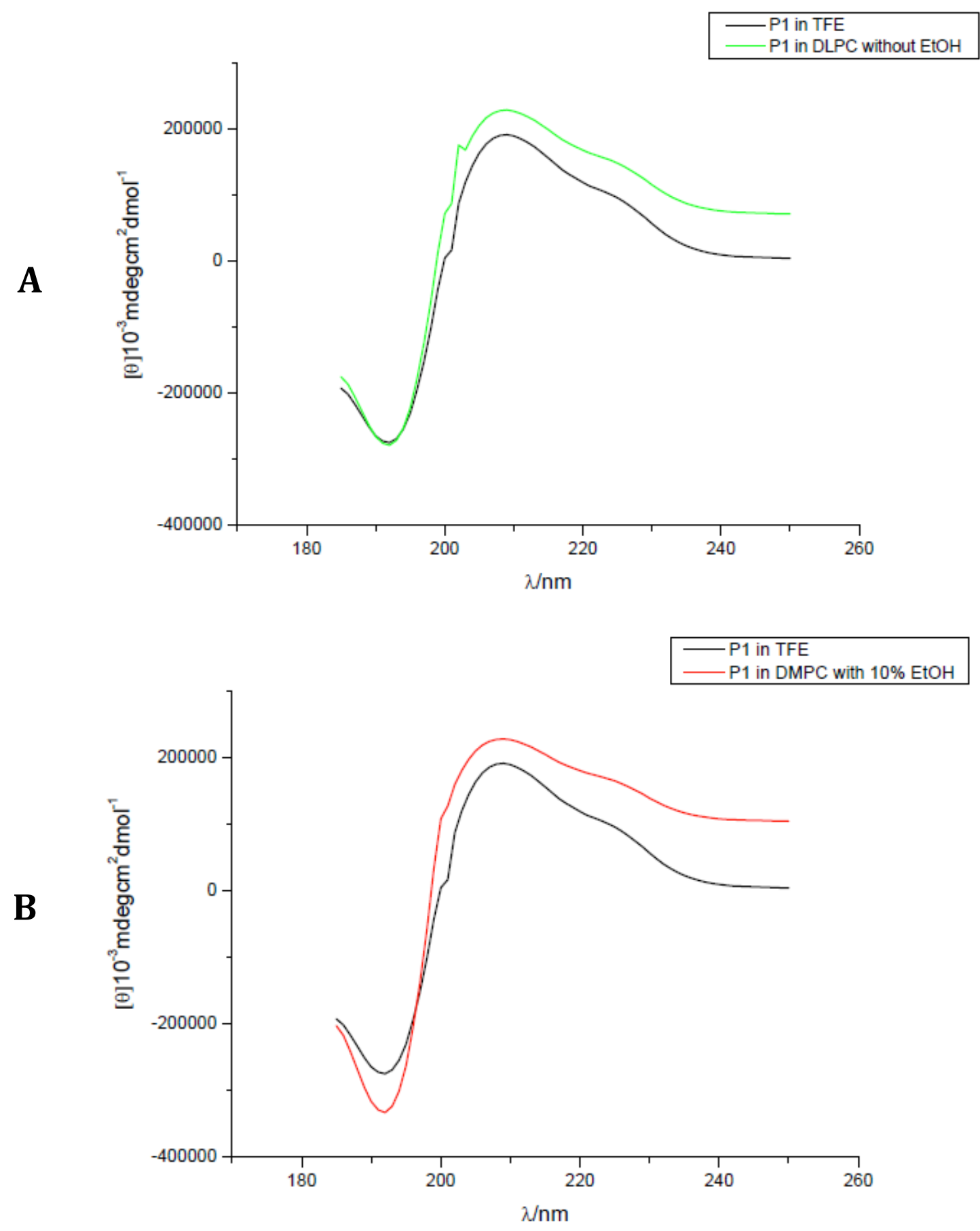


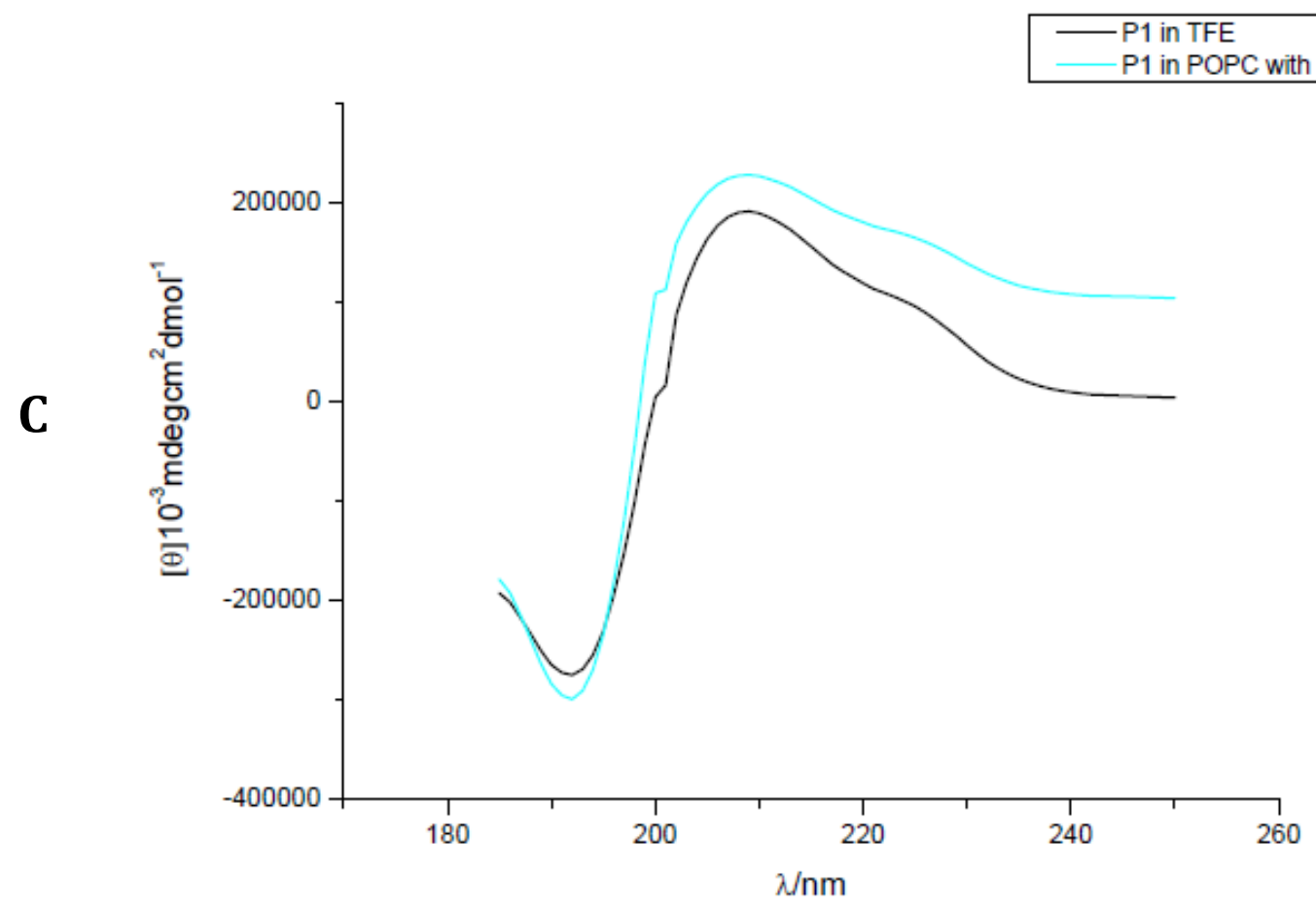

Figure 9.7: Comparison CD spectra of $\beta^{3}$ (Val/Ala/Leu triad) peptide P1 in TFE and in DLPC LUV without EtOH (A); in TFE and in DMPC LUV without and with 10\% EtOH, respectively (B); in TFE and in POPC LUV without and with $15 \%$ EtOH, respectively $(\boldsymbol{C})$

9.9 CD-spectra of $B^{3} / B^{2}$ (Val/Ala/Leu triad) peptide P2 in TFE and within three different lipid vesicles prepared in TRIS buffer keeping $P / L$ ratio 1:50 and peptide concentration $30 \mu \mathrm{M}$ constant at $50^{\circ} \mathrm{C}$ temperature

A

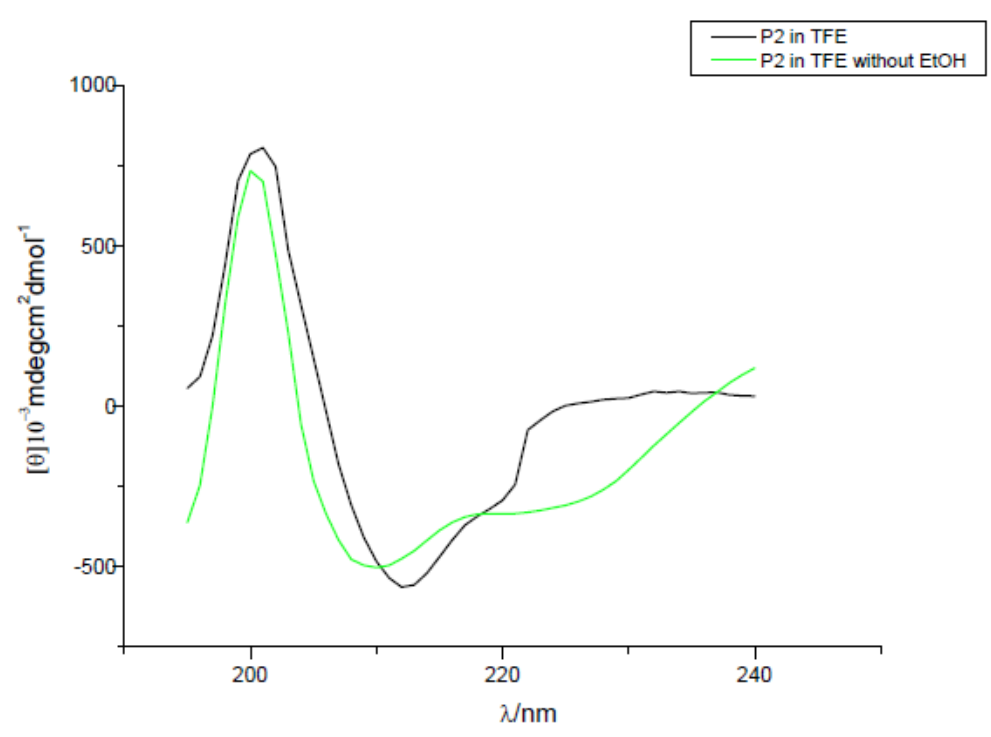



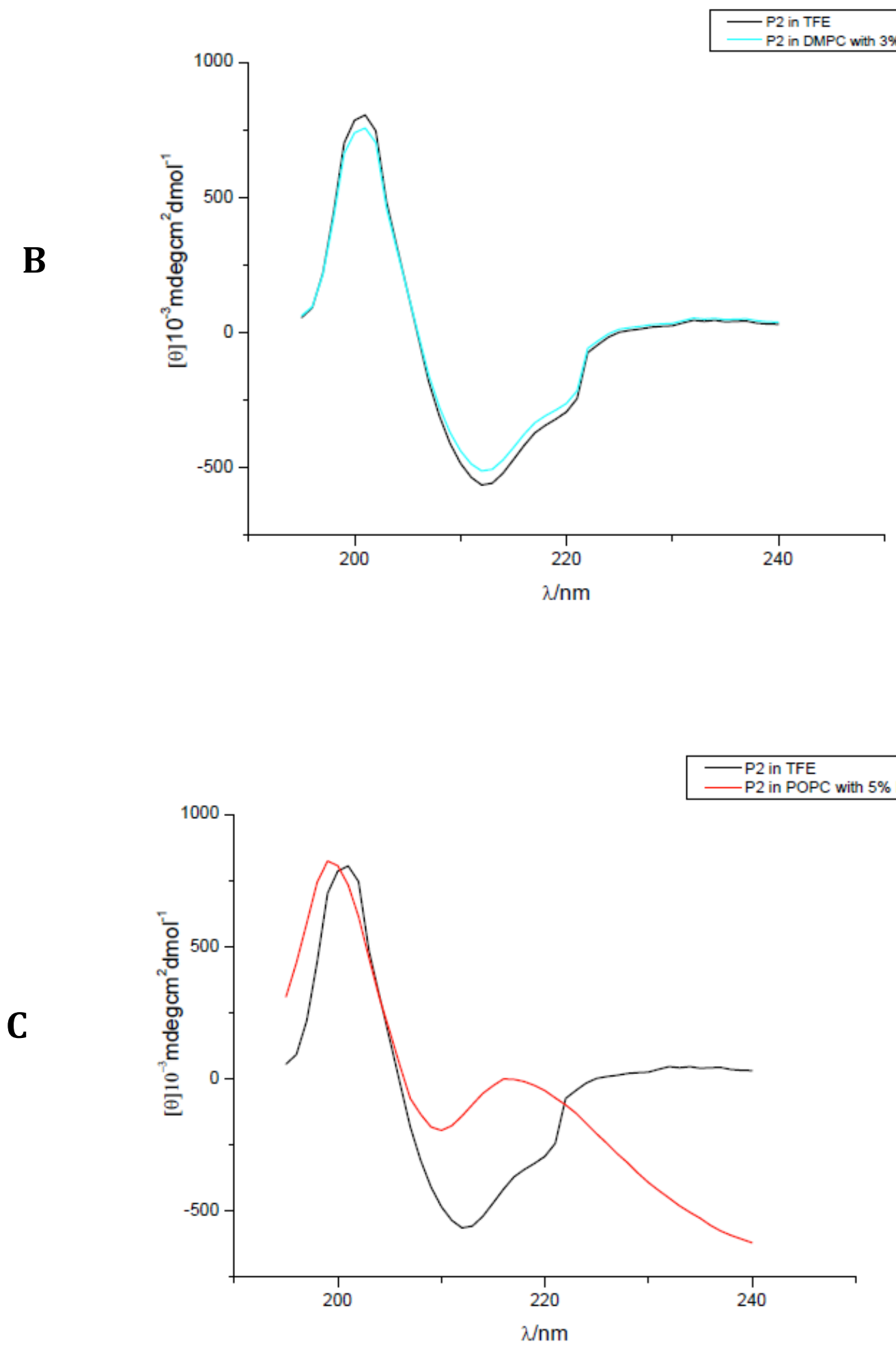

Figure 9.8: Comparison CD spectra of $\beta^{3} / \beta^{2}$ (Val/Ala/Leu triad) peptide P2 in TFE and in DLPC LUV without EtOH (A); in TFE and in DMPC LUV without and with 3\% EtOH, respectively (B); in TFE and in POPC LUV without and with 5\% EtOH, respectively (C) 
9.10 CD-spectra of $b^{2} / b^{3}$ (Val/Ala/Leu triad) peptide P3 in TFE and within three different lipid vesicles prepared in TRIS buffer keeping $P / L$ ratio 1:50 and peptide concentration $30 \mu \mathrm{M}$ constant at $50^{\circ} \mathrm{C}$ temperature

A
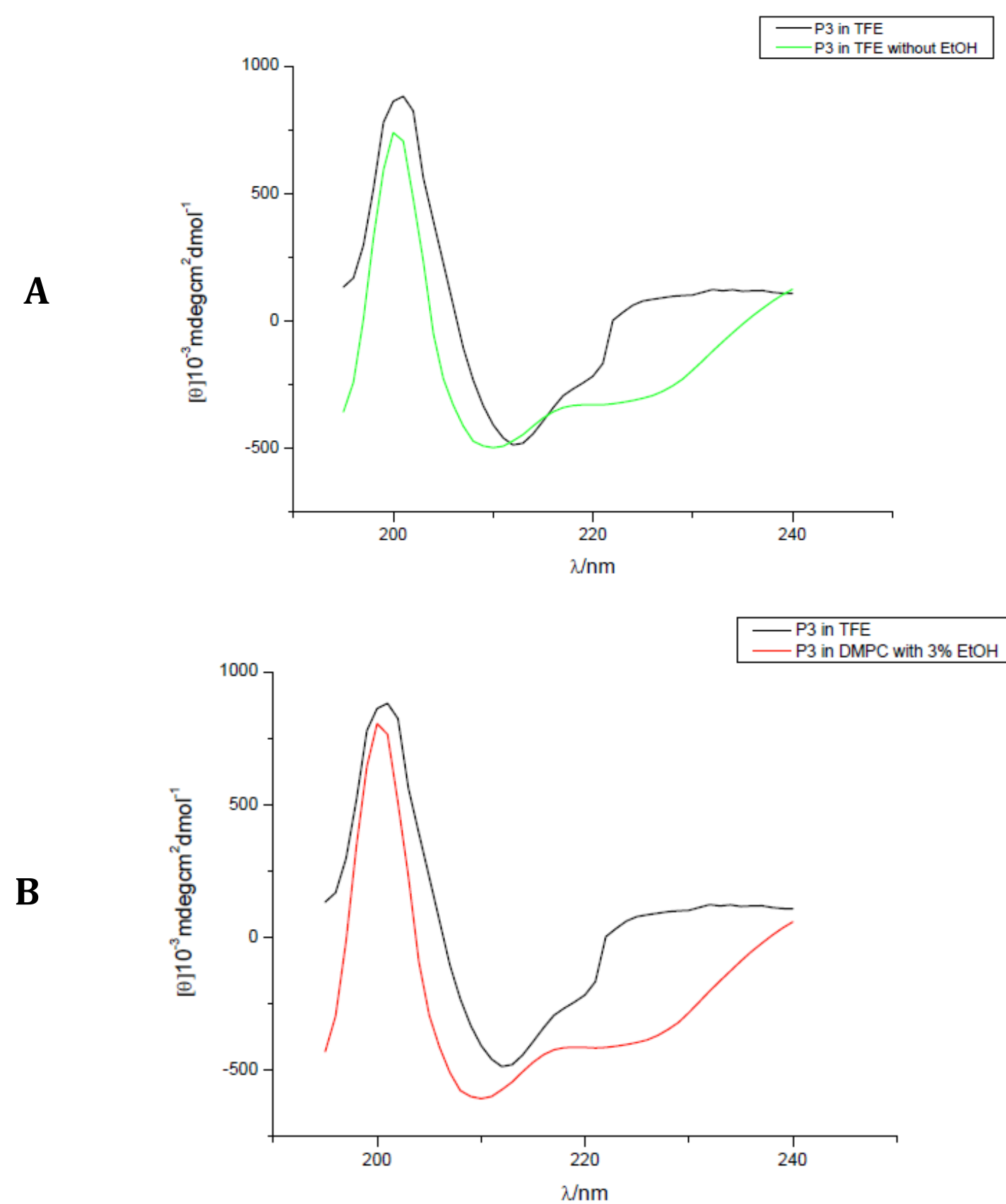


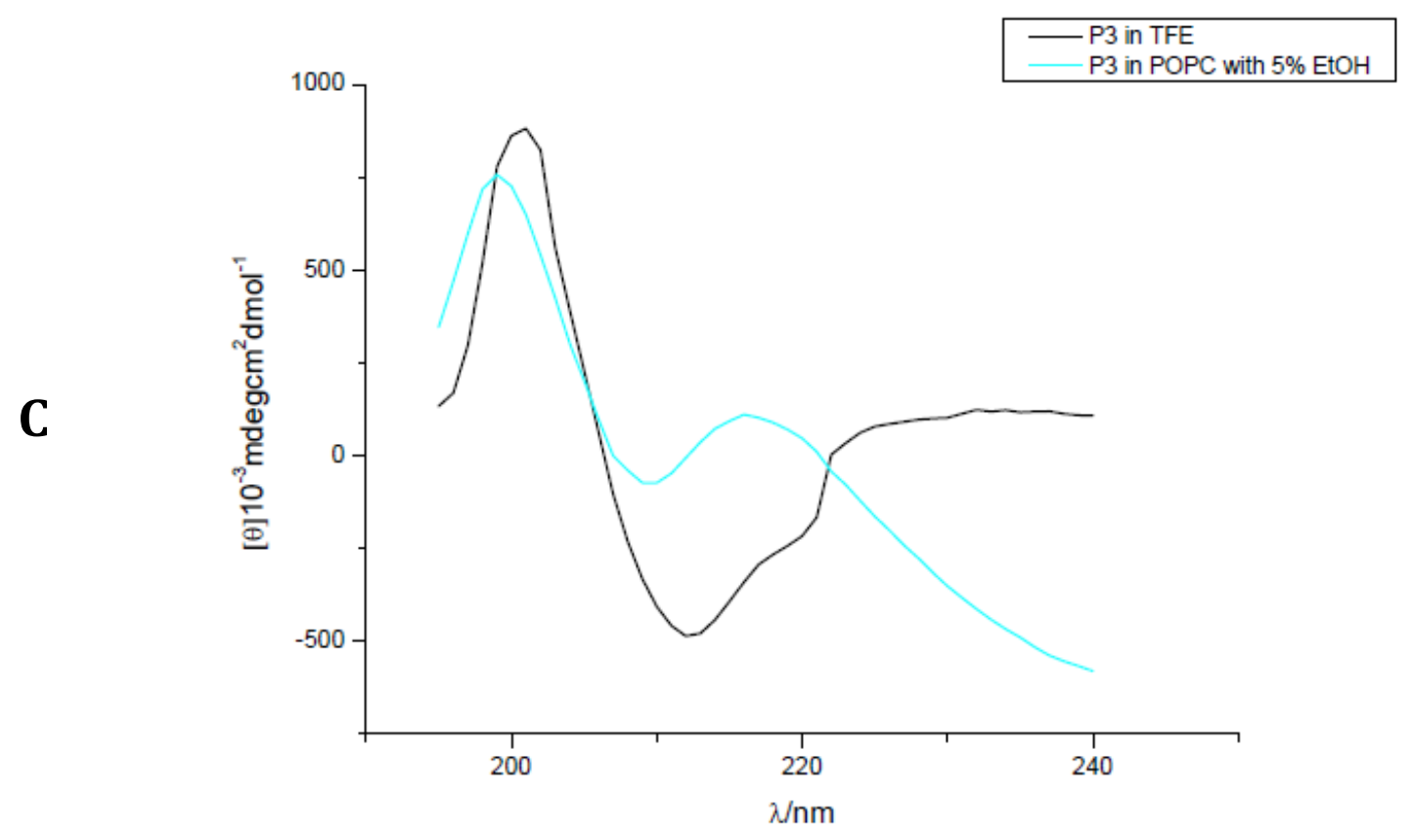

Figure 9.9: Comparison CD spectra of $\beta^{2} / \beta^{3}$ (Val/Ala/Leu triad) peptide P3 in TFE and in DLPC LUV without EtOH (A); in TFE and in DMPC LUV without and with 3\% EtOH, respectively (B); in TFE and in POPC LUV without and with 5\% EtOH, respectively (C)

7.11 CD-spectra of $b^{3}$ (Val) peptide P4 in TFE and within three different lipid vesicles prepared in TRIS buffer keeping $P / L$ ratio $1: 50$ and peptide concentration $30 \mu \mathrm{M}$ constant at $50^{\circ} \mathrm{C}$ temperature

A

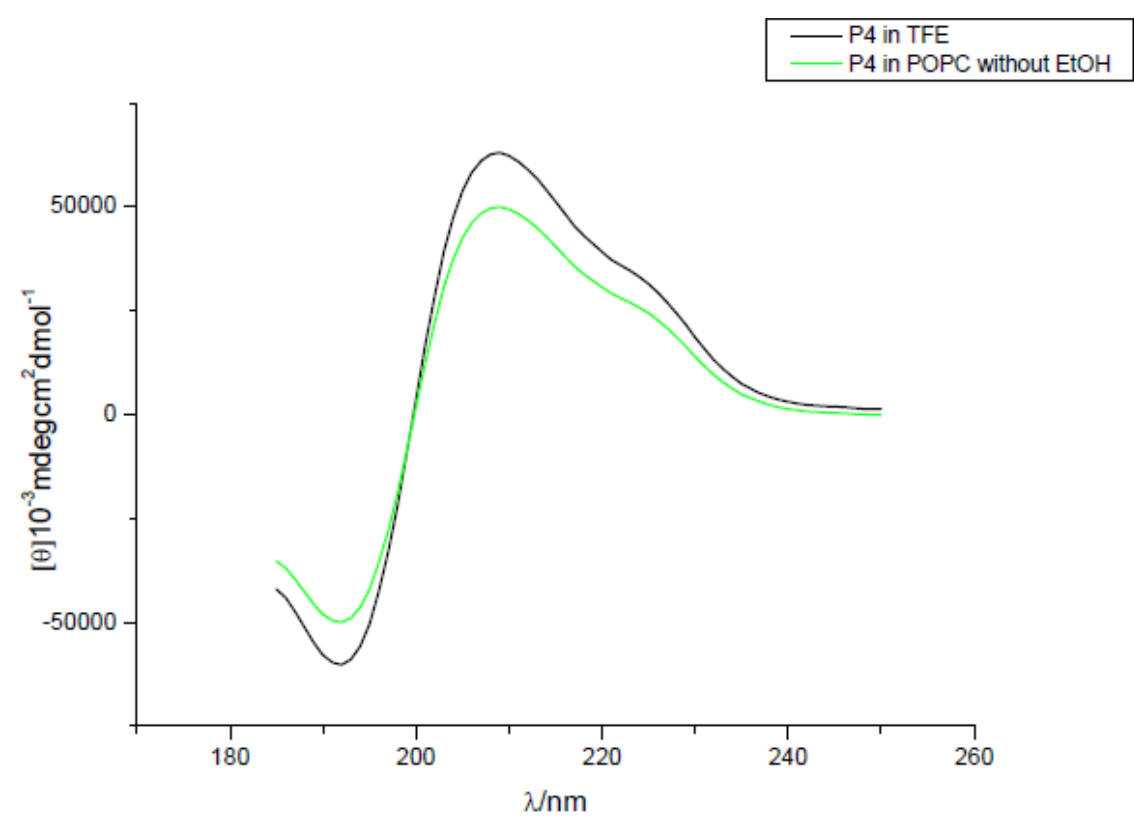



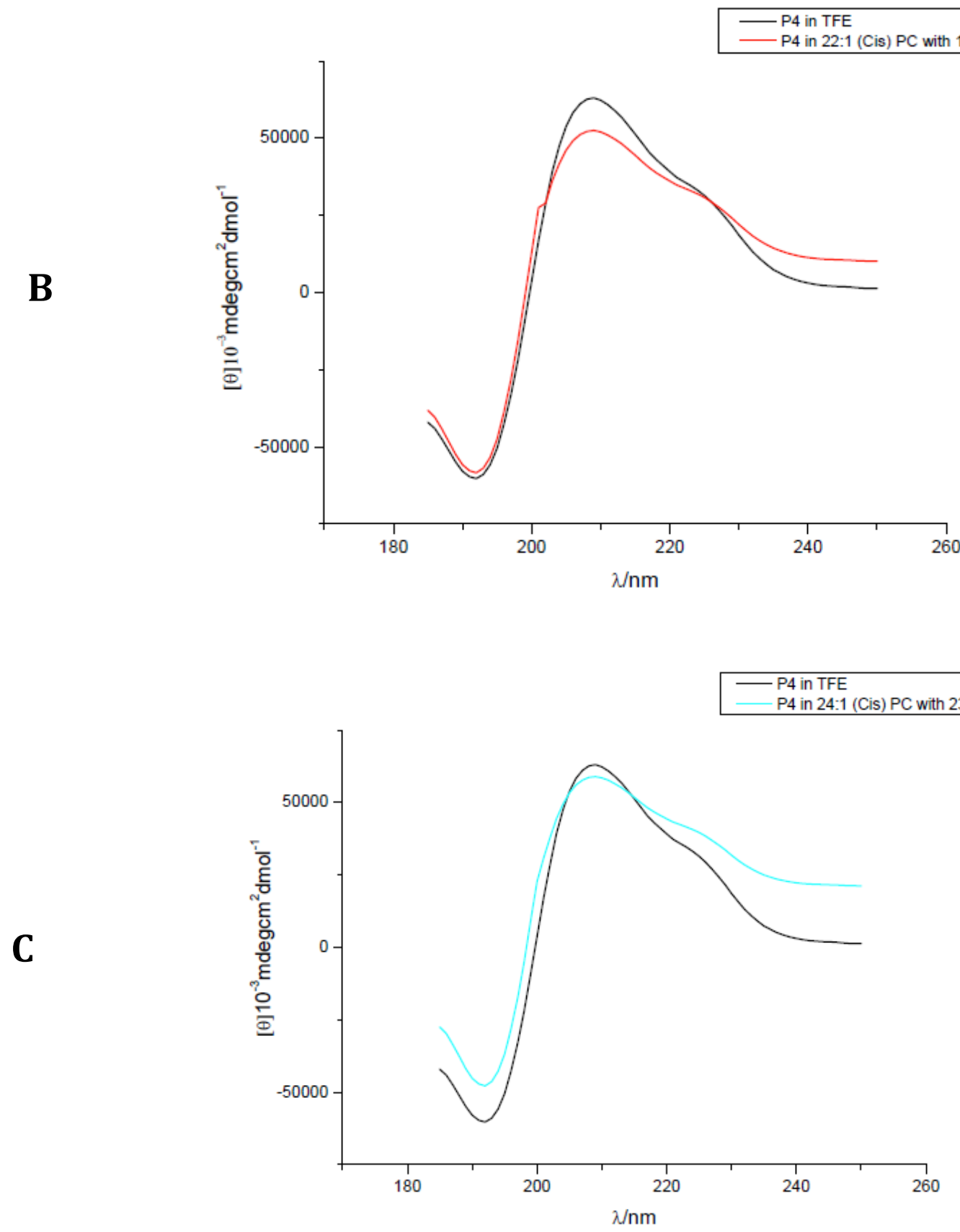

Figure 9.10: Comparison CD spectra of $\beta^{3}$ (Val) peptide P4 in TFE and in POPC LUV without EtOH (A); in TFE and in $\{22: 1$ (Cis) PC $\}$ LUV without and with 10\% EtOH, respectively (B); in TFE and in $\{24: 1$ (Cis) PC\} LUV without and with 23\% EtOH, respectively (C)

9.12 CD-spectra of $B^{3}$ (Leu) peptide P5 in TFE and within three different lipid vesicles prepared in TRIS buffer keeping $P / L$ ratio 1:50 and peptide concentration $30 \mu \mathrm{M}$ constant at $50^{\circ} \mathrm{C}$ temperature 


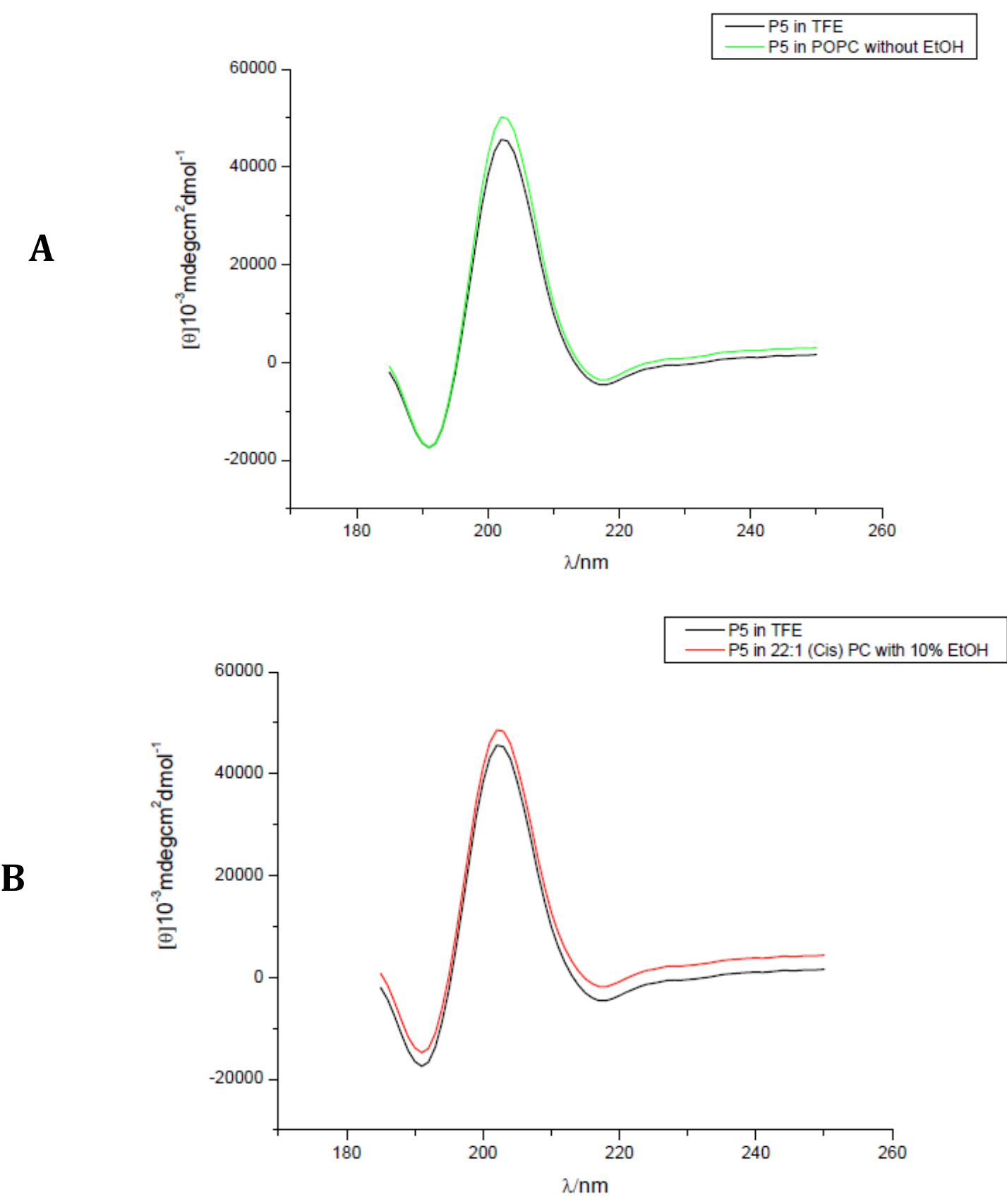




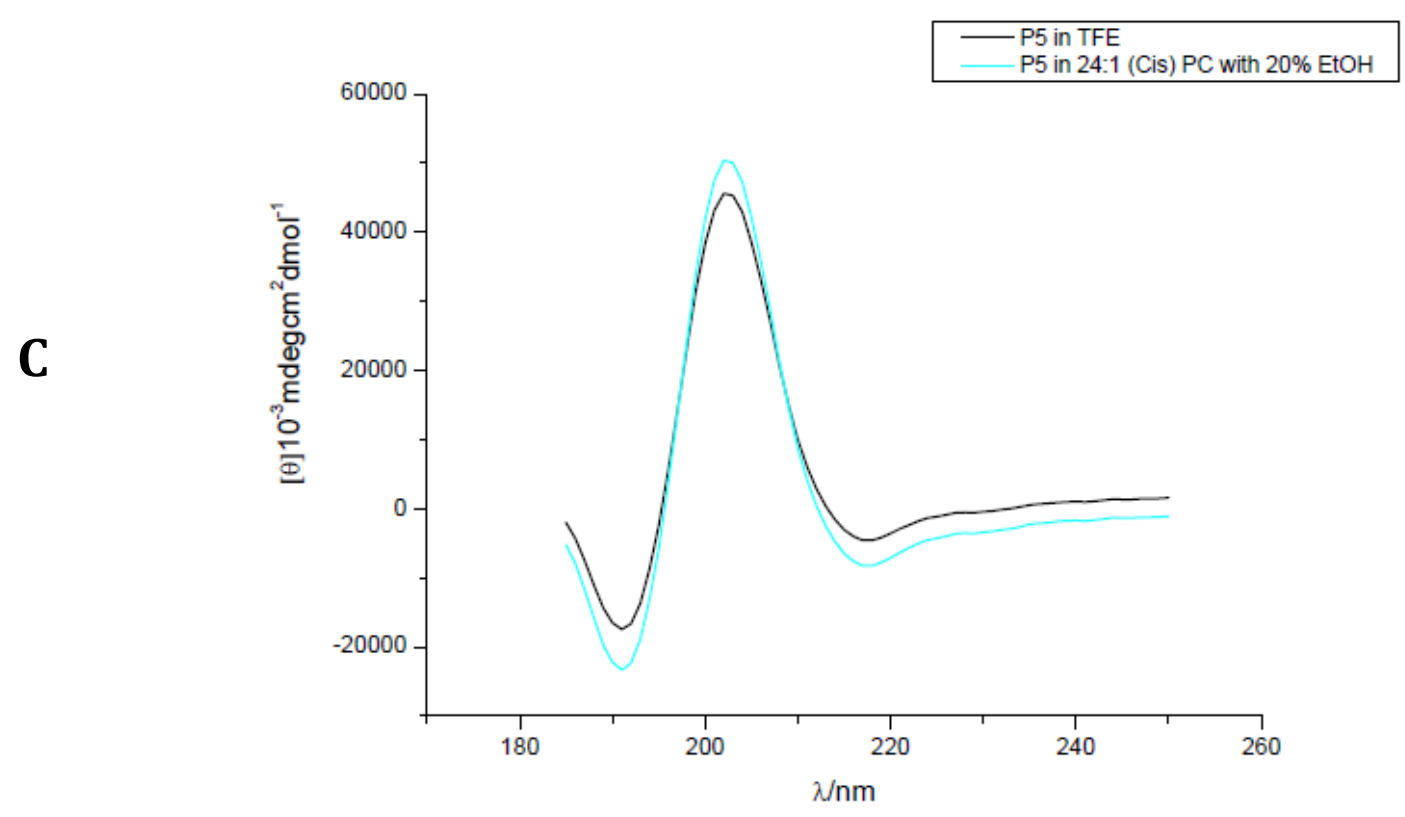

Figure 9.11: Comparison CD spectra of $\beta^{3}$ (Leu) peptide P5 in TFE and in POPC LUV without EtOH (A); in TFE and in $\{22: 1$ (Cis) PC $\}$ LUV without and with 10\% EtOH, respectively (B); in TFE and in $\{24: 1$ (Cis) PC\} LUV without and with 20\% EtOH, respectively (C) 


\section{Abbreviations}

$\AA$

AA

$\mathrm{AcOH}$

$\mathrm{Ac}_{2} \mathrm{O}$

ACHC

ACPC

APC

$\mathrm{aq}$

Avg

$\mathrm{Ar}$

Boc

${ }^{\circ} \mathrm{C}$

Capp

CBZ

CD

$\mathrm{CDCl}_{3}$

$\mathrm{CHCl}_{3}$

CPP

CLSM

$\mathrm{Cm}$

CPL

$\delta$

D

d

DBU

DCC

DCHC

DCM

Deg

DIC

DIPEA

DLPC angstrom $\left(10^{-8} \mathrm{~cm}\right)$

amino acid

acetic acid

acetic anhydride

trans-2-aminocyclohexanecarboxylic acid

trans-2-aminocyclopentanecarboxylic acid

trans-3- amino-pyrrolidine-4-carboxylic acid

aqueous

average

argon

tert-butoxycarbonyl

degree Celsius

capping

carboxybenzyl

circular dichroism

deuterated chloroform

chloroform

cell penetrating peptide

confocal laser scanning microscopy

centimeter

circularly polarized light

chemical shift

dextrorotatory

doublet

1,8-diazabicyclo[5.4.0] undec-7-en

diisopropylcarbodimide

trans-2,5 diaminocyclohexanecarboxylic acid

dichlormethane

degree

$N, N$-diisopropylcarbodiimide

$N, N$-diisopropylethylamine

1,2-dilauroyl-sn-glycero-3-phosphocholine 
DMPC

DMAP

DMF

DMSO

DMT

eq.

ESI

EtOAc

EtOH

$\mathrm{Et}_{2} \mathrm{O}$

Ex

FACS

FITC

FAM

FSPE

Fmoc

g

GC

GCMS

$\mathrm{h}$

HATU

HBTU

HCL

HFIP

HIV

HOAt

HOBt

HPLC

HR-MS

iPrOH

IR

Kcal

L

$\mathrm{LiCl}$

Lit. 1,2-dimyristoyl-sn-glycero-3-phosphocholine

4-Dimethylaminopyridine

$\mathrm{N}, \mathrm{N}$-dimethylformamide

dimethyl sulfoxide

$\mathrm{N}, \mathrm{N}$-Dimethyltryptamine

Equivalent

electrospray-ionisation

ethyl acetate

ethanol

diethyl ether

excitation

fluorescence-activated cell sorting

fluorescein isothiocyanate

Carboxyfluorescein

fluorous solid phase extraction

fluorenylmethoxycarbonyl

gram

gas chromatography

gas chromatography-mass spectroscopy

hour

$O$-(7-azabenzotriazol-1-yl)- $N, N, N^{\prime}, N^{\prime}$-tetramethyluronium hexafluorophosphate

$O$-(1H-benzotriazol-1-yl)- $N, N, N^{\prime}, N^{\prime}$-tetramethyluronium hexafluorophosphate

hydrochloric acid

hexafluoroisopropanol

human immunodeficiency virus

1-hydroxy-7-azabenzotriazole

1-hydroxybenzotriazole

high performance liquid chromatography

high-resolution mass spectrometry

isopropanol

infrared

kilocalorie

levorotatory

lithium chloride

Liter 
LL

LUV

$\lambda$

M

Max.

MeCN

Min.

$\mathrm{mm}$

$\mathrm{mL}$

mMol

$\mu \mathrm{mol}$

$\mu \mathrm{m}$

$\min$

MBHA

$\mathrm{MeOH}$

MF

$\mathrm{MHz}$

MLV

mw

M.W

$\mathrm{m} / \mathrm{z}$

$\mathrm{nm}$

NBD

NMM

NMP

NMR

NP

OSu

22:1 (Cis)PC

24:1 (Cis)PC

PCC

PEG

P:L

POPC

$\mathrm{pH}$

ppm low loaded

large unilamellar vesicle

wavelength

Molar

Maximum

acetonitrile

Minimum

milimeter

mililiter

milimolar

micromolar

micrometer

minute

4-methylbenzhydrylamine

methanol

mole fraction

megaharz

Multilamellar Vesicles

molecular weight

microwave

ratio of mass to charge

nanometer

7-nitrobenz-2-oxa-1,3-diazol-4-yl

$\mathrm{N}$-Methylmorpholine

$N$-methyl-pyrrolidone

nuclear magnetic resonance

normal phase

$\mathrm{N}-(9 \mathrm{H}-$ Fluorenylmethoxycarbonyloxy) succinimide

1,2-dierucoyl-sn-glycero-3-phosphocholine

1,2-dinervonoyl-sn-glycero-3-phosphocholine

pyridinium chlorochromate

polyethylene glycol

peptide-to-lipid

1-palmitoyl-2-oleoyl-sn-glycero-3-phosphocholine

the negative logarithm hydrogen-ion activity $\left(-\log _{10}\left[\mathrm{H}_{3} \mathrm{O}_{+}\right]\right)$

parts per million 


$\begin{array}{ll}\text { PyBOP } & \text { benzotriazole-1-yl-oxy-tris-pyrrolodino-phosphonium } \\ & \text { hexafluorophosphate } \\ \text { RP } & \text { reverse phase } \\ \text { RPM } & \text { rotations per minute } \\ \text { rt } & \text { room temperature } \\ \text { s } & \text { second } \\ \text { SPPS } & \text { solid phase peptide synthesis } \\ \text { SUV } & \text { small unilamellar vesicle } \\ \text { TAT } & \text { trans-activator of transcription } \\ \text { TFA } & \text { trifluoroacetic acid } \\ \text { TFE } & \text { trifluoroethanol } \\ \text { THF } & \text { tetrahydrofuran } \\ \text { TLC } & \text { thin-layer chromatography } \\ \text { TsO } & \text { tosyl } \\ t_{R} & \text { retention time } \\ T_{m} & \text { transition temperature } \\ \text { TRIS } & \text { trisaminomethane } \\ \text { Trt } & \text { trityl } \\ \text { UV } & \text { ultraviolet } \\ \text { V } & \text { volume } \\ \text { VIS } & \text { visual } \\ \text { W } & \text { watt } \\ & \\ & \\ & \end{array}$




\section{References}

1. Overton, E , Vjschr Naturf Ges Zurich, 1985, 40, 159-201.

2. E. Gorter, F. Grendel, Journal of Experimental Medicine, 1925, 41, 439-443.

3. M. Eeman, M. Deleu, Biotechnol. Agron. Soc. Environ. 2010, 14, 719-736.

4. S.J. Singer, G.L. Nicholson, Science, 1972, 175, 720-731.

5. J. H. Collier, P. B. Messersmith, Annu. Rev. Mater. Res. 2001, 31, 237-263.

6. F. Frézard, Braz, J. Med. Biol. Res. 1999, 32, 181-189.

7. R. Koynova, M. Caffrey, BBA Rev. Biomembr. 1998, 1376, 91-145.

8. N. Maurer, D. B. Fenske, P. R. Cullis, Expert Opin. Biol. Ther. 2001, 1, 923-947.

9. A. Akbarzadeh, R. Rezaei-Sadabady, S. Davaran, S. W. Joo, N. Zarghami, Y. Hanifehpour, M.

Samiei, M. Kouhi, K. Nejati-Koshki, Nanoscale Res. Lett., 2013, 8, 102.

10. N. Monteiro, A. Martins, R. L. Reis, N. M. Neves, J. R. Soc. Interface, 2014, 11

11. E. Wallin, G. von Heijne, Protein Sci. 1998, 7, 1029-1038.

12. G.C. Terstappen, A. Reggiani, Trends Pharmacol. Sci., 2001, 22, 23-26.

13. G. von Heijne, J. Intern. Med., 2007, 261, 543-557.

14. Y. Arinaminpathy, E. Khurana, D.M. Engelman, M.B. Gerstein, Drug Discov. Today, 2009, 14, 1130-1135.

15. H. Fan,W.M. Meng, C. Kilian, S. Grams, W. Reutter, Eur. J. Biochem., 1997, 246, 243-251.

16. M. Auer, G.A. Scarborough, W. Kuhlbrandt, Nature, 1998, 392, 840-843.

17. W. Kuhlbrandt, M. Auer, G.A. Scarborough, Curr. Opin. Struct. Biol. 1998 , 8, 510-516.

18. K.L. Pierce, R.T. Premont, R.J. Lefkowitz, Nat. Rev. Mol. Cell Biol., 2002, 3, 639-650.

19. J.J. Skehel, D.C. Wiley, Annu. Rev. Biochem., 2000, 69, 531-569.

20. T.E. Willnow, A. Nykjaer, J. Herz, Nat. Cell Biol., 1999, 1, E157-E162.

21. G. Yellen, Nature , 2002, 419, 35-42.

22. Y. Jiang, A. Lee, J. Chen, M. Cadene, B.T. Chait, R. MacKinnon, Nature, 2002, 417, 515-522.

23. F. Michelangeli, E.A. Grimes, J.M. East, A.G. Lee, Biochemistry, 1991, 30, 342-351.

24. R.J. Froud, C.R.A. Earl, J.M. East, A.G. Lee, Biochim. Biophys. Acta, 1986, 860, 354-360.

25. M. Cajrey, G.W. Feigenson, Biochemistry, 1981, 20, 1949-1961.

26. R.L. Cornea, D.D. Thomas, Biochemistry, 1994, 33, 2912-2920.

27. A. Johannsson, G.A. Smith, J.C. Metcalfe, Biochim. Biophys. Acta, 1981, 641, 416-421. 
28. C. Montecucco, G.A. Smith, F. Dabbeni-sala, A. Johansson, Y.M. Galante, R. Bisson, FEBS Lett., 1982, 144, 145-148.

29. G. In 't Veld, A.J. Driessen, J.A. Op den Kamp, W.N. Konings, Biochim. Biophys. Acta, 1991, $1065,203-212$.

30. R. George, R.N. Lewis, S. Mahajna, R.N. McElhaney, J. Biol. Chem., 1989, 264, 11598-11604.

31. M.F. Brown, Chem. Phys. Lipids, 1994, 73, 159-180.

32. J. Le Coutre, L.R. Narasimhan, C. Kumar, N. Patel, H.R. Kaback, Proc. Natl. Acad. Sci. USA, 1997, 94, 10167-10171.

33. O.P. Karlsson, M. Rytomaa, A. Dahlqvist, P.K. Kinnunen, A. Wieslander, Biochemistry 1996, 35, 10094-10102.

34. R. Epand, Biochim. Biophys. Acta, 1998, 1376, 353-363.

35. R.S. Cantor, Biochemistry (1997) 2339^2344.

36. A.G. Lee, Biochim. Biophys. Acta., 1998, 1376, 381-390.

37. N.J. Ryba, D. Marsh, Biochemistry, 1992, 31, 7511-7518.

38. L.R. Maneri, P.S., J. Biol. Chem., 1988, 263, 16170-16178.

39. L.R. Maneri, P.S. Low, Biochem. Biophys. Res. Commun., 1989, 159, 1012-1019.

40. S. Morein, J. A. Killian, and M. M. Sperotto, Biophys. J, 2002, 82, 1405-1417.

41. E. Strandberg, S. Ozdirekcan, D. T. S. Rijkers, P. C. A. van der Wel, R. E. Koeppe, R. M. J. Liskamp, and J. A. Killian, Biophys. J., 2004, 86, 3709-3721.

42. F. Liu, R. N. A. H. Lewis, R. S. Hodges, and R. N. McElhaney, Biochemistry, 2001, 40, 760-768.

43. F. Liu, R. N. A. H. Lewis, R. S. Hodges, and R. N. McElhaney, Biochemistry, 2002, 41, $9197-$ 9207.

44. F. Liu, R. N. A. H. Lewis, R. S. Hodges, and R. N. McElhaney, Biochemistry, 2004, 43, 36793687.

45. B.Y. van Duyl, D. T. S. Rijkers, B. de Kruijff, and J. A. Killian, FEBS Lett., 2002, 523, 79-84.

46. G.A. Caputo and E. London, Biochemistry, 2003, 42, 3275-3285.

47. W. K. Subczynski, M. Pasenkiewicz-Gierula, R. N. McElhaney, J. S. Hyde, and A. Kusumi, Biochemistry, 2003, 42, 3939-3948.

48. R. N. A. H Lewis, Y. P. Zhang, R. S. Hodges, W. K. Subczynski, A. Kusumi, C. R. Flach, R. Mendelsohn, and R. N. McElhaney, Biochemistry, 2001, 40, 12103-12111.

49. M. R. R., de Planque, B. B. Bonev, J. A. A. Demmers, D. V. Greathouse, R. E. Koeppe, F. Separovic, A. Watts, and J. A. Killian, Biochemistry, 2003, 42, 5341-5348.

50. A. Ridder, W. van de Hoef, J. Stam, A. Kuhn, B. de Kruijff, and J. A. Killian, Biochemistry, 2002, $41,4946-4952$. 
51. E. Strandberg, S. Morein, D. T. S. Rijkers, R. M. J. Liskamp, P. C. A. van der Wel, and J. A. Killian. 2002, Biochemistry, 41:7190-7198.

52. T. Bystrom, G. Grobner, and G. Lindblom, Colloids Surf. A Physicochem. Eng. Asp, 2003, 228, $37-42$.

53. M. R. R. de Planque and J. A. Killian, Mol. Membr. Biol. 2003, 20, 271-284.

54. L. L. Holte and K. Gawrisch, Biochemistry, 1997, 36, 4669-4674.

55. S. E. Feller, C. A. Brown, D. T. Nizza and K. Gawrisch, Biophys. J., 2002, 82, 1396-1404.

56. J. A. Barry, and K. Gawrisch, Biochemistry, 1994, 33, 8082-8088.

57. U. Vierl, L. Lobbecke, N. Nagel and G. Cevc, Biophys. J., 1994, 67, 1067-1079.

58. H. V. Ly and M. L. Longo, Langmuir, 2002, 18, 8988-8995.

59. H. V. Ly and M. L. Longo, Biophys. J., 2004, 87, 1013-1033.

60. P. Seeman, Pharmacol. Rev, 1972, 24, 583.

61. J. L. Slater and C.-H. Huang, Prog. Lipid Res., 1988, 27, 325.

62. U. Vierl, L. Lobbecke, N. Nagel and G. Cevc, Biophys. J., 1994, 67, 1067

63. S. J. Ryhanen, J. M. I. Alakoskela and P. K. J. Kinnunen, Langmuir 2005, 21, 5707.

64. E. S. Rowe, Biochemistry, 1983, 22, 3299.

65. E. S. Rowe, Biochim. Biophys. Acta, 1985, 813, 321.

66. E. S. Rowe, Biochemistry, 1987, 26, 46.

67. S. A. Simon and T. J. McIntosh, Biochim. Biophys. Acta, 1984, 773, 169.

68. S. A. Simon, T. J. McIntosh and M. L Hines, Molecular and Cellular Mechanisms of Anaesthesia, Plenum Press: New York, 1986, p 297

69. J. L Slater and C.-H. Huang, In: The Structure of Biological Membranes; P. L. Yeagle, Ed., CRC Press: New York, 2005, p 121.

70. S. H. Gellman, Acc. Chem. Res. 1998, 31, 173-180

71. J. McKee and T. McKee, Biochemistry; The Molecular Basis of Life. Oxford University Press, Inc. 2014.

72. J. Kovacs, R. Ballina, R. L. Rodin, D. Balasubramanian and J. Applequist, J. Am. Chem. Soc. 1965, 87, 119-120.

73a. D. Seebach, M. Overhand, F. N. M. Ku“hnle, B. Martinoni, L. Oberer, U. Hommel, and H. Widmer, Helv. Chim. Acta, 1996, 79, 913.

73b. D. Seebach, P. E. Ciceri, M. Overhand, B. Jaun, D. Rigo, L. Oberer, U. Hommel, R. Amstutz and H. Widmer, Helv. Chim. Acta, 1996, 79, 2043.

73c. T. Hintermann and D. Seebach, Synlett, 1997, 437.

73d. T.Hintermann and D. Seebach, Chimia, 1997, 51, 244.

73e. G. Guichard and D. Seebach, Chimia, 1997, 51, 315. 
74a. G. P. Dado and S. H. Gellman, J. Am. Chem. Soc., 1994, 116, 1054.

74b. D. H. Appella, L. A. Christianson, I. L. Karle, D. R. Powell and S. H. Gellman,. J. Am. Chem. Soc,. 1996, 118, 13071.

74c. D. H. Appella, L. A. Christianson, D. A. Klein, D. R. Powell, X. Huang, J. J. Barchi and S. H. Gellman, Nature, 1997, 387, 381.

74d. S. Krauthäuser, L. A. Christianson, D. R. Powell and S. H. Gellman, J. Am. Chem. Soc., 1997, $119,11719$.

75a. B. L Verson, Nature, 1997, 385, 113.

75b. S. Borman, Chem. Eng. New, June 16, 1997, 32.

76. D. Seebach, S. Abele, J. V. Schreiber, B. Martinoni, A. K. Nussbaum, H. Schild, H Schulz, H. Hennecke, R. Woessner and F. Bitsch, Chimia, 1998, 52, 734-739.

77. M. D. Disney, D. F. Hook, K. Namoto, P. H. Seeberger, D. Seebach, Chem. Biodiversity, 2005, 2, 1624-1634.

78. D. Seebach, K. Namoto, Y. R. Mahajan, P. Bindsch“adler, R. Sustmann, M. Kirsch,

N. S. Ryder, M. Weiss, M. Sauer, C. Roth, S. Werner, H.-D. Beer, C. Mundling,

P. Walde and M. Voser, Chem. Biodiversity, 2004, 1, 65-97.

79. E. A. Porter, X. F W.ang, H. S. Lee, B. Weisblum and S. H., Gellman, Nature 2000, 404, 565.

80. E. A.; orter, B. Weisblum and S. H. Gellman, J. Am. Chem. Soc., 2002, 124, 7324-7330.

81. B. P. Mowery, S. E. Lee, D. A. Kissounko, R. F. Epand, R. M. Epand, B. Weisblum,

S. S. Stahl and S. H. Gellman, J. Am. Chem. Soc., 2007, 129, 15474-15476.

82. K. Gademann and D. Seebach, Helv. Chim. Acta, 2001, 84, 2924-2937.

83. J. Kritzer, J. D. Lear, M. E. Hodsdon and A. Schepartz, J. Am. Chem. Soc., 2004, 126, 9468-9469.

84. J. A. Kritzer, M. E. Hodsdon and A. Schepartz, J. Am. Chem. Soc., 2005, 127, 4118-4119.

85. N. Trouche, S. Wieckowski, W. Sun, O. Chaloin, J. Hoebeke, S. Fournel and

G. Guichard, J. Am. Chem. Soc. 2007, 129, 13480-13492.

86. O. Stephens, S. Kim, B. D. Welch, M. E. Hodsdon, M. S. Kay and A. Schepartz,

J. Am. Chem. Soc., 2005, 127, 13126-13127.

87. J. S. Kritzer, O. M. Stephens, D. A. Guarracino, S. K. Reznik and A. Schepartz, Bioorg. Med. Chem., 2005, 13, 11-16.

88. T. Kimmerlin, K. Namoto and, D. Seebach, Helv. Chim. Acta, 2003, 86, 2104-2109.

89. K. Namoto, J. Gardiner, T. Kimmerlin and D. Seebach, Helv. Chim. Acta, 2006, 89, 3087-3103.

90. M. A. Gelman, S. Richter, H. Cao, N. Umezawa, S. H. Gellman and T. M. Rana, Org. Lett,. 2003, 5, 3563-3565.

91. T. Shimanouchi, P. Walde, J. Gardiner, Y. R. Mahajan, D. Seebach, A.Thomae,

S. D. Kramer, M. Voser and R. Kuboi, Biochim. Biophys. Acta: Biomembr., 2007, 1768, 
$2726-2736$.

92. Y.-D. Wu, W. HA, D. P. Wang, Y. Gao and Y. L. Zhao, Acc. Chem. Res., 2008, 41, 1418-1427

93. D.Seebach, S. Abele, K. Gademann, G. Guichard, T. Hintermann, B. Jaun, J. L. Matthews, J. V., Schreiber, L. Oberer, U. Hommel and H. Widmer, Helv. Chim. Acta., 1998, 81, 932

94. D. H. Appella, L. A. Christianson, I. L. Karle, D. R. Powel and S.H., Gellman, J. Am. Chem. Soc., 1996, 118, 13071.

95. D. H. Appella, L. A. Christianson, I. L. Karle, D. R. Powel and S.H., Gellman, J. Am. Chem. Soc. ,1999, 121, 6206-6212.

96. D. H. Appella, P. R. LePlae, T. L. Raguse and S. H. Gellman, J. Org. Chem., 2000, 65, 4766.

97. D. H. Appella, J. J. Barchi, S. R. Durell and S. H. Gellman, J. Am. Chem. Soc., 1999, 121, 2309.

98. D. H. Appella, L. A. Christianson, D. A. Klein, D. R. Powell, X. Huang, J. J. Barchi and S. H. Gellman, Nature, 1997, 387, 381.

99. J. Applequist, K. A. Bode, D. H. Appella, L. A. Christianson and S. A. Gellman, J. Am. Chem. Soc., 1998, 120, 4891.

100. X. Wang J. F. Espinosa and S.H. Gellman, J. Am. Chem. Soc. 2000, 122, 4821.

101. R.P. Cheng, S. H. Gellman and W. F. DeGrado, Chem. Rev. 2001, 101, 3219-3232

102. G. Quinkert, E. Egert and C. Griesinger, In: Peptides: Synthesis, Structure, and Applications; Gutte, Ed. Academic Press: SanDiego, 1995.

103. D. Seebach and J. L. Matthews, Chem. Commun., 1997, 2015-2022

104. R. F. Epand, L. Raguse, S. H. Gellman and R. M. Epand, Biochemistry (Mosc.), 2004, 43, 95279535.

105. P. Chakraborty and U. Diederichsen, Chem. Eur. J. 2005, 11, 3207-3216.

106. A. M. Brückner, P. Chakraborty, S. H. Gellman and U. Diederichsen, Angew. Chem. Int. Ed. 2003, 42, 4395-4399.

107. B. W. Gung, D. Zou, A. M. Stalcup and C. E. Cottrell, J. Org. Chem. 1999, 64, 2176-2177.

108. Y. Hamuro, J. P. Schneider and W. F. DeGrado, J. Am. Chem. Soc. 1999, 121, 12200-12201.

109. T. L. Raguse, J. R. Lai, P. R. LePlae and S. H. Gellman, Org. Lett. 2001, 3, 3963-3966.

110. D. H. Appella, L. A. Christianson, D. A. Klein, M. R. Richards, D. R. Powell and S. H. Gellman, J. Am. Chem. Soc. 1999, 121, 7574.

111. D. Seebach, K. Gademann, J. V. Schreiber, J. L. Matthews, T. Hintermann, B. Jaun, L. Oberer, U. Hommel and H. Widmer, Helv. Chim. Acta 1997, 80, 2033.

112. E. Sparr, W. L. Ash, P. V. Nazarov, D. T. S. Rijkers, M.A. Hemminga, D. P. Tieleman and J. A. Killian, The Journal of Biological Chemistry, 2005, 280 (47), 39324-39331

113. C. A. Miller, J. P. Hernández-Ortiz, N. L. Abbott, S. H. Gellman and J. J. de Pablo, The Journal of Chemical Physics, 2008, 129, 15102

114. W.J G. Hol, Prog. Biophys. Molec. Biol, 1985, 45 149-195. 
115. C. A. Miller, S. H. Gellman, N. L. Abbott and J. J. de Pablo, Biophysical Journal, 2009, 96, 4349-4362

116. D. W. Urry, Proc. Nat. Acad. Sci. USA, 1972, 69 (6), 1610-1614,

117. K. H. Sippel and F. A. Quiocho, Protein Science, 2015, 24, 1040-1046

118. K. A. Bode and J. Applequist, Macromolecules, 1997, 30, 2144-2150.

119. T. E. Creighton, Proteins, 2nd ed. W. H. Freeman and Co. New York, 1993.

120. E. Wallin and G. von Heijne, Protein Sci., 1998, 7 (4), 1029-38.

121. A. Krogh, B. Larsson, G. von Heijne and E.L. Sonnhammer, J Mol Biol. 2001, 305 (3), 567-80.

122. J.P. Overington , B .Al-Lazikani , A.L. Hopkins , Nat Rev Drug Discov. 2006, 5 (12), 993-6.

123. O.G. Mouritsen and M. Bloom, Biophys. J., 1984, 46, 141-53.

124. D. M. Engelman, T.A. Steitz and A. Goldman, Annu. Rev. Biophys. Biophys. Chem. 1986, 15, 321-53.

125. J.A. Killian, Biochim. Biophys. Acta, 1998, 1376, 401-15.

126. J.A. Killian and G. von Heijne, Trends Biochem. Sci., 2000, 25, 429-34.

127. M. Monne, I. Nilsson, M. Johansson, N. Elmhed and G. von Heijne, J. Mol. Biol. 1998, 284, 1177-83.

128. P. Braun and G. von Heijne, Biochemistry, 1999, 38, 9778-82.

129. A.N.J.A. Ridder, S. Morein, J.G. Stam, A. Kuhn, B. de Kruijff and J.A. Killian, Biochemistry, 2000, 39, 6521-8.

130. K. Hristova, W.C. Wimley, V.K. Mishra, G.M. Anantharamiah, J.P. Segrest and S.H. White, J. Mol. Biol. 1999, 290, 99-117.

131. M. Schiffer, C.H. Chang and F.J. Stevens, Protein Eng., 1992, 5, 213.

132. R. A. Reithmeier, Curr. Opin. Struct. Biol. 1995, 5, 491-500.

133. J. Deisenhofer and H. Michel, EMBO J., 1989, 8, 2149-70.

134. D.A. Doyle, J.M. Cabral, R.A. Pfuetzner, A.L. Kuo, J.M. Gulbis, S.L. Cohen, B.T. Chait and R. Mackinnon, Science, 1998, 280, 69-77.

135. S.W. Cowan, T. Schirmer, G. Rummel, M. Steiert, R. Ghosh, R.A. Pauptit, J.N. Jansonius and J.P. Rosenbusch, Nature, 1992, 358, 727-33.

136. F. Kovacs, J. Quine and, T.A. Cross, Proc. Natl. Acad. Sci. U. S. A., 1999, 96, 7910-5.

137. A.S. Ladokhin, M.E. Selsted and S.H. White, Biophys. J., 1997, 72, 794-805.

138. W.C. Wimley and S.H. White, Nat. Struct. Biol., 1996, 3, 842-8.

139. M.R.R. de Planque, J.A.W. Kruijtzer, R.M.J. Liskamp, D. Marsh, D.V. Greathouse, R.E. II Koeppe, B. de Kruijff, J.A. and Killian, J. Biol. Chem., 1999, 274, 20839-46.

140. G. von Heijne, Nature, 1989, 341, 456-8. 
141. G. V. Miloshevsky and J. P. Jordan, Trends Neurosci., 2004, 27, 308-314.

142. R. E. Koeppe and O. S. Andersen, Biophys. Biomol. Struct., 1996, 25, 231-258.

143. R. Sarges and B. Witkop, J. Am. Chem. Soc., 1965, 87, 2011-2020.

144. S. B. Hladky and D. A. Haydon, Biochim. Biophys. Acta, 1972, 274, 294-312.

145. R. Wieduwild, M. Tsurkan, K. Chwalek, P. Murawala, M. Nowak, U. Freudenberg, C. Neinhuis, C. Werner and Y. Zhang J. Am. Chem. Soc., 2013, 135 (8), 2919-2922.

146. Y. Chi and S. H. Gellman, J. Am. Chem. Soc. 2006, 128, $6804-6805$.

147. Y. Chi, E. P. English, W. C. Pomerantz, S. Horne, L. A. Joyce, L. R. Alexander, W. S. Fleming, E. A. Hopkins and S.H. Gellmann, J. Am. Chem. Soc. 2007, 129, 6050 - 6055.

148. G. Nagula, V. J. Huber, C. Lum and B. A. Goodman, Org. Letters, 2000, 2 (22), 3527-3529.

149. A. G. Myers, B. H. Yang, H. Chen and J. L. Gleason, J. Am. Chem. Soc. 1994, 116, $9361-9362$.

150. A. G. Myers, J. L. Gleason and T. Yoon, J. Am. Chem. Soc. 1995, 117, 8488 - 8489.

151. A. G. Myers and L. McKinstry, J. Org. Chem. 1996, 61, 2428 - 2440.

152. A. G. Myers, J. L. Gleason, T. Yoon and D.W. Kung, J. Am. Chem. Soc.,1997, 119, 656 - 673.

153. A. G. Myers, B. H. Yang, H. Chen, L. McKinstry, D. J. Kopecky and J. L. Gleason, J. Am. Chem. Soc., 1997, 119, $6496-6511$

154. D. A. Kummer, W. J. Chain, M. R. Morales, O. Quiroga and A. G. Myers, J. Am. Chem. Soc., 2008, $130,13231-13233$.

155. J. A. Kritzer, J. Tirado-Rives, S. A. Hart, J. D. Lear, W. L. Jorgensen and A. Schepartz, J. Am. Chem. Soc., 2005, 127, 167-178.

156. J. S. Park, H. S. Lee, J. R. Lai, B. M. Kim and S. H. Gellman, J. Am. Chem. Soc., 2003, 125, 85398545.

157. T. L. Raguse, J. R. Lai and S. H. Gellman, Helv. Chim. Acta, 2002, 85, 4154-4164.

158. P. I. Arvidsson, J. Frackenpohl, D. Seebach, Helv. Chim. Acta, 2003, 86, 1522-1553.

159. O. Flögel, J. D. C. Code, D. Seebach, and P. H. Seeberger, Angew. Chem. Int. Ed. 2006, 45, 7000 -7003 .

160. B. Kolesinska, K. K. Rozniakowski, J. Fraczyk, I. Relich, A. Maria papini and Z. J. Kaminski. Eur. J. Org. Chem, 2015, 2015(2), 401-408

161. S. M. Kelly and N. C. Price, Curr. Prot. Pept. Sci., 2000, 1, 349-384.

162. G.D. Fasman (Ed.), Circular Dichroism and the Conformational Analysis of Biomolecules, Plenum Press, New York, 1996.

163. N. Berova, K. Nakanishi and R. W. Woody (Eds.), Circular Dichroism: Principles and Applications, 2nd ed, Wiley-VCH, New York, 2000.

164. A. Rodger and B. Nordén, Circular Dichroism and Linear Dichroism, Oxford Univ. Press, Oxford, 1997. 
165. J. W. Nelson and N. R. Kallenbach, Proteins 1986, 1, 211-217.

166. D. M. Engelman and B. A. Lewis, J. Mol. Biol., 1983, 166, 211-217.

167. I. Martin, M. Teixido and E. Giralt, Pharmaceuticals, 2010, 3: 1456-1490.

168. V. Torchilin, Technologies, 2008 5: 95-103.

169. S. Pujals and E. Giralt, Adv Drug Deliv Rev, 2008, 60: 473-484.

170. A, Elmquist, M. Lindgren, T. Bartfai and U. Langel, Exp Cell Res, 2001, 269, 237-244

171. G. Manish and S. Vimukta, Res J Chem Sci, 2011, 1, 135-138.

172. B. Wang, T. J. Siahaan and R. Soltero, Drug Delivery: Principles and Applications., 2005.

173. H. Kamada T. Okamoto M. Kawamura, H. Shibata and Y. Abe, Biol Pharm Bull, 2007, 30, 218-223.

174. M. Zorko and U. Langel, Advanced Drug Delivery Reviews, 2005, 57, 529-545.

175. S. Fawell, J. Seery, Y. Daikh, C. Moore and L.L. Chen, Proc Natl Acad Sci U S A , 1994, 91, 664668.

176. L.N. Johnson, S.M. Cashman and R. Kumar-Singh, Mol. Ther., 2008, 16, 107-114.

177. Y.L. Chiu, A. Ali, C.Y. Chu, H. Cao, T.M. Rana, Chem Biol, 2004, 11, 1165-1175.

178. J. J. Turner, G. D., Ivanova, B. Verbeure, D. Williams and A.A. Arzumanov, Nucleic Acids Res, 2005, 33, 6837- 6849 .

179. J.B. Rothbard, S. Garlington, Q. Lin, T. Kirschberg and E. Kreider, Nat Med, 2000, 6, 12531257.

180. H.Yukawa, Y. Kagami, M. Watanabe, K. Oishi and Y. Miyamoto, Biomaterials, 2010, 31, 40944103

181. M. Lewin, N. Carlesso , C.H. Tung, X. W. Tang and D. Cory, Nat Biotechnol, 2000, 18, 410414.

182. J. Hoyer and I. Neundorf, Acc Chem Res, 2012, 45, 1048-1056.

183. F. Mussbach, M. Franke, A. Zoch , B. Schaefer and S. Reissmann, J Cell Biochem. 2011, 112, 3824-3833.

184. V. Kersemans and B. Cornelissen, Pharmaceuticals, 2010, 3, 600-620.

185. K. Saar, M. Lindgren, M. Hansen, E. Eiriksdottir, Y. Jiang, , K. Rosenthal-Aizman, M. Sassian, and U. Langel, Anal. Biochem., 2005, 345, 55-65.

186. D. Fischer, Y. Li, B. Ahlemeyer, J. Krieglstein, and T. Kissel, Biomaterials, 2003, 24, 1121-1131.

187. Y.Z. Lin, S. Yao, R. A Veach, T. R. Torgerson, and J. Hawiger, J. Biol. Chem., 1995 270, $14255-14258$. 
188. R. Fischer, T. Waizenegger, K. Köhler and R. Brock, Biochim. Biophys. Acta, Biomembr., 2002, $1564,365-374$.

189. C.Y. Jiao, D. Delaroche, F. Burlina, I.D. Alves, G. Chassaing, and S. Sagan, J. Biol. Chem., 2009, 284, 33957-33965.

190. F. Duchardt, M. Fotin-Mleczek, H. Schwarz, R. Fischer and R. Brock, Traffic, 2007, 8, 848866.

191. S. El-Andaloussi, P. Jarver, H. J. Johansson and U. Langel, Biochem. J. , 2007, 407, 285-292.

192. D. Derossi, S. alvet, A. Trembleau, A. Brunissen, G. Chassaing, and A. Prochiantz, J. Biol. Chem. ,1996, 271, 18188-18193.

193. I.D. Alves, N. Goasdoue, I. Correia, S. Aubry, C. Galanth, S. Sagan, , S. Lavielle , and G. Chassaing,. Biochim. Biophys. Acta, 2008, 1780, 948-959.

194. P. Joanne, C. Galanth, N. Goasdoué, P. Nicolas, S. Sagan, S. Lavielle, G. Chassaing, and I. D. Alves, Biochim. Biophys. Acta, 2009, 1788, 1772-1781.

195. H. Hirose, T. Takeuchi, H. Osakada, S. Pujals, S. Katayama, I. Nakase, S. Kobayashi, T. Haraguchi, and S. Futaki, Mol. Ther., 2012, 20(5), 984-993.

196. A. Lamaziere, F. Burlina, C. Wolf, G. Chassaing, G. Trugnan, and J. Ayala- Sanmartin, PLoS ONE, $20072, \mathrm{e} 201$.

197. H. J. Ryser, and R. Hancock, Science, 1965, 150, 501- 503.

198. P.A. Wender, D.J. Mitchell, K. Pattabiraman, E.T. Pelkey, L. Steinman, and J.B. Rothbard, Proc. Natl. Acad. Sci. USA, 2000, 97, 13003-13008.

199. D. J. Mitchell, D.T. Kim, L. Steinman, C.G. Fathman and J.B. Rothbard, Pept. Res., 2000, 56, 318-325.

200. J.B. Rothbard, T. C. Jessop, R. S. Lewis, B.A. Murray, and P.A. Wender, J. Am. Chem. Soc., 2004, 126, 9506-9507.

201. I. Nakase, T. Takeuchi, G. Tanaka, and S . Futaki, Adv. Drug Deliv. Rev., 2008, 60, 598-607.

202. P.A. Wender, W.C. Galliher, E.A. Goun, L.R Jones, and T.H. Pillow, Adv. Drug Deliv. Rev. ,2008, $60,452-472$

203. H.D. Herce, and A.E. Garcia, Proc. Natl. Acad. Sci. USA, 2007, 104, 20805-20810.

204. H.D., Herce A.E. Garcia, J. Litt, R.S. Kane, P. Martin, N. Enrique, A. Rebolledo and V. Milesi, Biophys. J., 2009, 97, 1917-1925.

205. E. Barany-Wallje, J. Gaur, P. Lundberg, U. Langel and A. Graslund, FEBS Lett., 2007, 581, 2389-2393.

206. S. Deshayes, T. Plenat, P. Charnet, G. Divita, G. Molle and F. Heitz, Biochem. Biophys. Acta, 2006, 1758, 1846-1851. 
207. L. E. Yandek, A. Pokorny, A. Floren, K. Knoelke, U. Langel, and P.F. Almeida, Biophys. J. 2007, $92,2434-2444$.

208. P.F. Almeida and A. Pokorny, Biochemistry, 2009 48, 8083-8093.

209. C. Palm-Apergi, A. Lorents, K. Padari, M. Pooga, and M. Hallbrink, FASEB J., 2009, 23, 214223.

210. B. Chérine, S. Sandrine, FEBS Letters, 2013, 587, 1693-1702

211. T. T.Puck and P.I. Marcus, J. Exp. Med. 1956, 103, 653-666.

212. T. Bereau and M. J. Deserno, Chem. Phys., 2009, 130 (23), 235106.

213. S. Matysiak and P. Das, Phys. Rev. Lett., 2013, 111, 058103.

214. J. N. Onuchic, Z. Luthey-Schulten and P. G. Wolynes, Annu. Rev. Phys. Chem., 1997, 48, 545600.

215. W. G. J. Hol, L. M Halie. and C. Sander, Nature, 1981, 294, 532-536.

216. J Frackenpohl, P. I. Arvidsson, J. V. Schreiber, D. Seebach, Chembiochem, 2001, 2, 445-455. 217. Spectrochimica Acta Part A 51 (1995) L7-L21

218. M. Gude, J. Ryf, P. D White, Lett. Pept. Sci. 2002, 9, 203-206.

219. N. J. Greenfield, Nat. Protoc. 2007, 1, 2876-2890.

220. G. Guichard, S. Abele, D. Seebach, Helv. Chim. Acta 1998, 81, 187-206.

221. P. I. Arvidsson, J. Frackenpohl, D. Seebach, Helv. Chim. Acta 2003, 86, 1522-1553.

222. Chi, Y., Gellman, S. H., J. Am. Chem. Soc. 2006, 128, $6804-6805$.

223. Chi, Y., English, E. P., Pomerantz, W. C., Horne, S., Joyce, L. A., Alexander, L. R., Fleming, W. S., J. Am. Chem. Soc. 2007, 129, 6050.

224. Beata Kolesinska, Klaus Eyer, Tom Robinson, Petra S. Dittrich, Albert K. Beck, Dieter Seebach and Peter Walde. Chemistry \& Biodiversity, 2015, 12, 697-732

225. P. I. Arvidsson, J. Frackenpohl, D. Seebach, Helv. Chim. Acta 2003, 86, 1522-1553.

226. B. Blankemeyer-Menge, M. Nimtz, R. Frank, Tetrahedron Lett. 1990, 31, 1701-1704 227. J. Mol. Biol. (1983) 166, 211-217

228. N. Kučerka, M. P. Nieh, J. Katsaras Biochim. Biophys. Acta2011, 1808, 2761-2771.229. R. C. MacDonald, R. I. MacDonald, B. P. M. Menco, K. Takeshita, N. K. Subbarao, L. Hu, Bio-chim. Biophys. Acta BBA - Biomembr. 1991, 1061, 297-303.230. Curran, D. P.; Hadida, S.; He, M. J. Org. Chem. 1997, 62, 6714-6715.

231. W. Zhang, D. P. Curran / Tetrahedron 62 (2006) 11837-11865 


\section{Acknowledgement}

I would like to pay my sincere gratitude and convey warm regards to my primary supervisor Prof. Dr. Ulf Diederichsen for his cooperation and support all through my journey towards achieving doctoral degree. I already received the constant support right from the beginning during applying for a suitable doctoral scholarship at German Academic Exchange Service (DAAD). Later on the same enthusiastic active cooperation continued throughout these four years during my stay at AK Diederichsen.

I would also like to convey my deep regards to my co-supervisor Prof. Dr. Claudia Steinem for her involvement into my project and guiding me to the right direction in the research work. I would definitely like to thank Dr. Bastian Rouven Brückner, Dr. Tabea Oswald and Frau Angela Rübeling for their active cooperation and help in materializing the experiments with cell lines. Without their active participation and involvement, the cell culture work would not have been possible. I would also like to convey my deep regards to Dr. Franziska Thomas for her encouraging support.

I am very tahnkful to Frau Aoife Neville for helping me out with all the official works required during first two years. I am extremely fortunate to have Frau Angela Heinemann to be there always for me, not only helping me out with all kinds of official works, but also for the moral support all along.

I would alos like to name Martin Börsken, as my labmate and a very close friend, who has always been a support in the lab. I would also like to thank all the other group members for making my stay at AK Diederichsen so cherishing.

I thank Daniel Frank for helping me out with the technical issues. I also thank Dr. Frauendorf at the Mass department and Mr. John at the NMR department for their kind support in measuring my samples. 
I would like to thank German Academic Exchange Service (DAAD) for supporting me financially during my doctoral studies. I am also tahnkful to SFB 803 for giving me the excellent opportunity to work in A01 project. I would also like to thank IOBC, Uni-Göttingen.

Last but not the least; I am ever thankful to my beloved parents, my lovely wife and my friendphilosopher-guide, grandpa \& grandma, for their constant support, encouragement and love.

I thank God for this wonderful life. 


\section{Curriculum Vitae}

Personal Attributes

Name:

Amartya Banerjee

Date of Birth:

08.07.1989

Place of Birth:

Asansol, India

Nationality:

Indian

Academic Education

$10 / 2014-09 / 2018$

$06 / 2014-09 / 2014$

$08 / 2011-07 / 2013$
Doctoral thesis (PhD) in the research group of Prof. Dr.

Ulf Diederichsen at Institute of Organic and Biomolecular Chemictry, Georg-August University, Göttingen with fully funded scholarship from German Academic Exchange Service (DAAD)

Thesis Title: 'Beta Peptide as Transmembrane Domains: Aggregation, Recognition and Lipid-Peptide Aggregation

Mandatory German language course at Goethe Institute, Göttingen

Level: $\mathbf{B} 1.2$

Masters' Degree (M.Sc) in Chemistry with Organic Chemistry as specialization at Department of Chemistry, Visva Bharati Central University, India

Thesis Title: 'Theoretical investigation on Gold (III) catalyzed isomerization of substituted allenes to conjugated dienes using a nitroso compound as additive'

Final Grade: GPA - 7.6 (out of 10.0 ), First Class with Distinction 
$07 / 2008-05 / 2011$

Work Experience
Bachelors' Degree (B.Sc Honours) in Chemistry at Department of Chemistry, Visva Bharati Central University, India

Thesis Title: ` Towards the synthesis of unnatural amino acids'

Final Grade: GPA - 73.6\%, First Class with Distinction
06/2018 - To Date

$10 / 2014-05 / 2018$

$03 / 2015-02 / 2017$

$04 / 2016-12 / 2016$
Chemical Engineer at Marabu GmbH \& Co. KG, Tamm, Stuttgart Region

$>$ Expertise in UV printing inks (screen printing) in the field of label printing (low migration), food packaging and glass printing UV / LED, Rotative screen printing, Raw material optimization / cost optimization.

$>$ Research and development in solvent-based inks

$>$ Developing new recepies for solvent-based inks for credit card lamination

Doctoral research fellow at Institute of Organic and Biomolecular Chemistry, Georg-August University, Göttingen

Planning and execution of research schemes

Accomplishing targets within time frame

$>$ Regular updates and presentation of results

$>$ Teaching andsupervising bachelor (Biochemistry) students in lab courses

Online Tutor (Chemistry) at Chegg Inc.

Tutoring school level, high school level and graduate level students with Chemistry related problems

Freelance Scientific Editor at Cactus Communications

Proofreading, copyediting and evaluating, modifying and rewriting scientific contexts 
Recognition

Languages \& Computational Skills

1. Bengali (Native)
$\begin{aligned} & \text { 2. Hindi (Native) } \\ & \text { 3. English (Business Fluent) } \\ & \text { 4. German (Intermediate, Level B1.2) }\end{aligned}$

Computational Skills MS Office Package, Origin 8.5, ChemBio Draw, Sci Finder, Molden, Pymol, Gaussian 09, Gaussview

Hobby

Listening to music, singing Indian classical music

05.08.2018, Göttingen

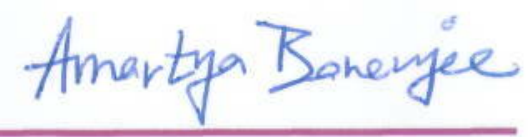

Date/Place

Signature 